\title{
Automatic Arterial Wall Detection and Diameter Tracking using M-mode Ultrasound
}

\author{
By \\ Shanmugaraja Krishnasamy Venugopal
}

A thesis submitted to the Faculty of Graduate and Postdoctoral Affairs in partial

fulfillment of the requirements for the degree of

Master of Applied Science

in

Electrical and Computer Engineering

Carleton University,

Ottawa, Ontario, Canada

(C) 2019

Shanmugaraja Krishnasamy Venugopal 


\section{Abstract}

Research indicate that the ultrasonic monitoring of the artery stiffness aids in early detection of coronary artery disease. One of the challenges in monitoring the stiffness of the artery in mass screening tests is the need for a clinical expert to identify the artery and to estimate the change in diameter in an ultrasound image. In this research, an algorithm to automatically detect artery wall region and estimate systolic and diastolic lumen diameter of the artery is proposed using ultrasound radio-frequency signals acquired with a single ultrasound transducer in M-mode. Detection of the region containing the artery walls is obtained using a machine learning trained classifier. Artery inner walls are identified in the detected region using correlation and peak detection and the lumen diameter is then estimated. The proposed algorithm is first tested on phantom experiments using a clinical ultrasound imaging system with a linear array ultrasonic transducer (UT) probe and then tested on the M-mode signal acquired with human in-vivo subjects using a wearable ultrasound sensor (WUS) composed of a single flexible UT. The machine learning-based artery wall region classifier was able to detect the artery wall region in M-mode signals acquired from the clinical ultrasound acquisition system with an accuracy of $88.9 \%$. The classifier achieved an accuracy of $88.3 \%$ in the case of data acquired from WUS. Further, the diameter estimated in the human in-vivo experiment with the proposed technique was within the range of the average diameter of the common carotid artery observed in humans. Thus, the study offers a method for automatic lumen diameter estimation of the artery in M-mode ultrasound signals. The proposed technique will be useful in developing an artery health monitoring system using ultrasound. 


\section{Acknowledgement}

I would like to express my deep and sincere gratitude to the people who have helped me in the completion of the thesis.

I would first like to thank my thesis supervisors Prof. Yuu Ono and Prof. Sreeraman Rajan for their constant support, and encouragement. I am genuinely grateful to Prof. Ono, for providing me this research opportunity and patiently guiding me through every phase of my thesis. I am particularly indebted to Prof. Rajan, who spent countless number of hours in helping me understand research methods and find solutions. I am forever indebted for this opportunity and experience.

I would like to thank Dr. Masayoshi Yoshida for his guidance in the data acquisition and data segmentation process during the experiments. His insights and guidance in vascular ultrasound imaging and segmentation was very helpful.

I would like to extend my gratitude to Fatemeh Zabihollahy, who has been extremely supportive in helping me understand research concepts and exchanging ideas. I would also like to acknowledge Carleton University for the opportunity and financial support provided for the research.

I am grateful to my friends, Alexander Fernandes, Dipayan Mitra, Elliot Yeung, Tony Mathew, Anand, Ramnath, Ram Kishore, Sarath, Vignesh Prabhu, Emelina and Sanu who helped in all the possible ways. I would also like to thank my well-wishers Mark Jowett and Vijay Ratnavel who have been a constant source of moral support and encouragement.

Finally, I must express my very profound gratitude to my parents, brother and grandmother for providing me with unfailing support and continuous encouragement 
through the process of researching and writing this thesis. This accomplishment would not have been possible without them. 


\section{Table of Contents}

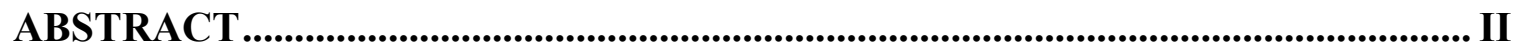

ACKNOWLEDGEMENT ...........................................................................................III

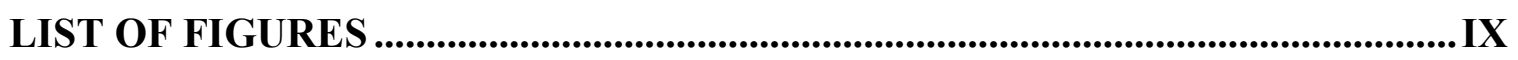

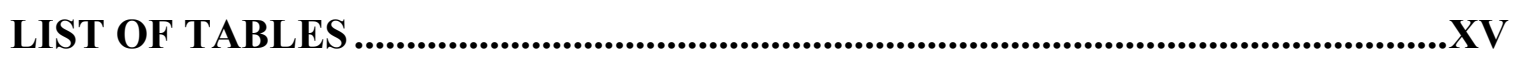

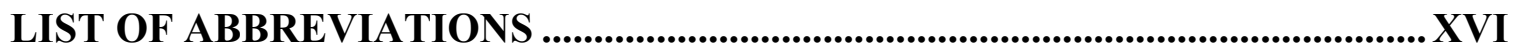

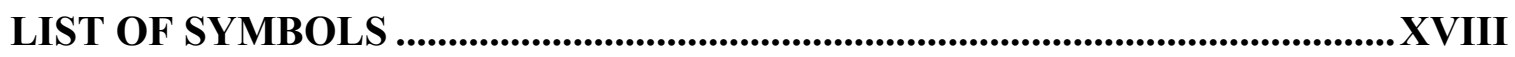

Chapter 1: Introduction .................................................................................................... 1

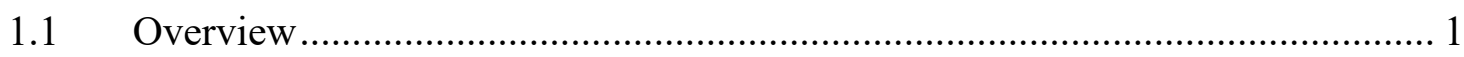

1.2 Problem statement.................................................................................. 3

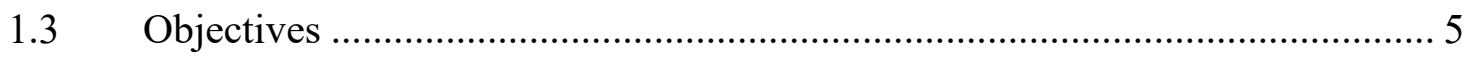

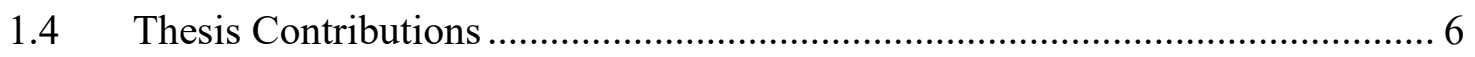

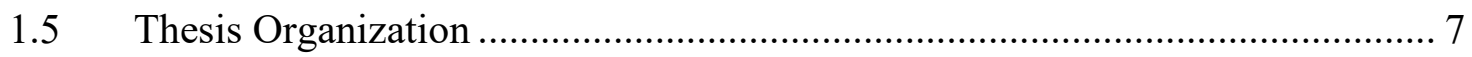

Chapter 2: Background Review ......................................................................................... 9

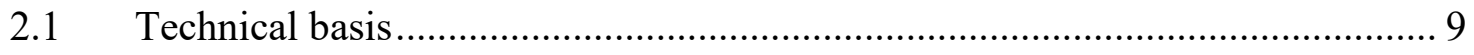

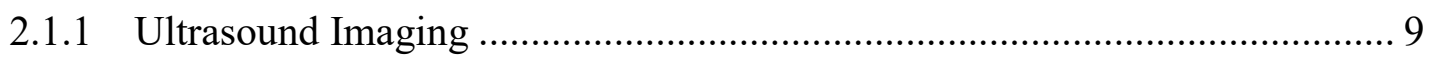

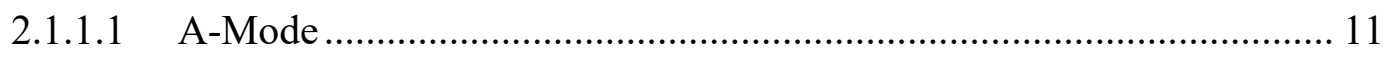

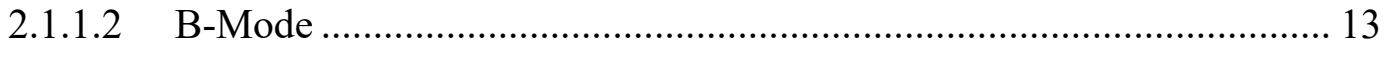

2.1.1.3 M-Mode ............................................................................ 15 
2.1.2 Review of machine learning methods used in this study .......................... 16

2.1.2.1 Linear Discriminant Analysis (LDA) ......................................... 17

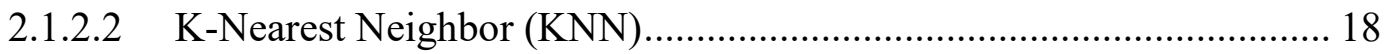

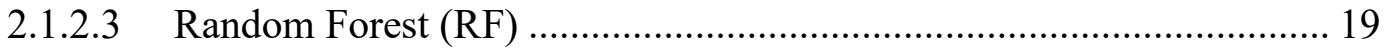

2.1.2.4 Extreme Gradient Boosting (XGB) ............................................ 21

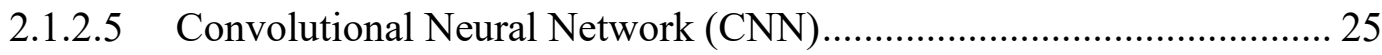

2.2 Artery structure and mechanical property............................................ 27

2.2.1 Composition of the arterial wall .......................................................... 27

2.2.2 Common Carotid Artery .................................................................... 28

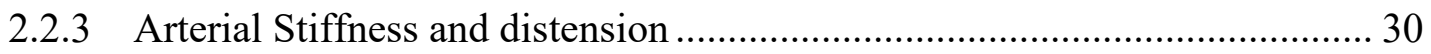

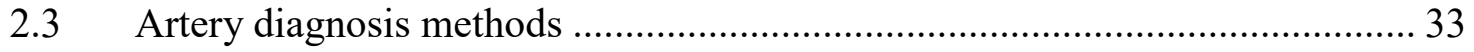

2.3.1 Endothelial dysfunction and FMD measurement .................................. 33

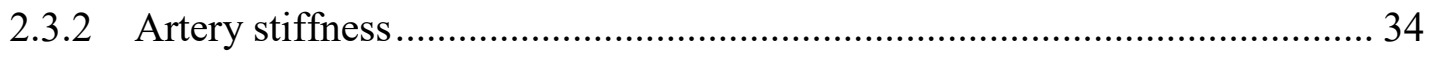

2.4 Measurement methods of artery diameter................................................. 36

2.4.1 Ultrasound measurement methods ...................................................... 36

2.4.1.1 B-mode ultrasound imaging ....................................................... 36

2.4.1.2 M-mode ultrasound image using a single UT ................................ 37

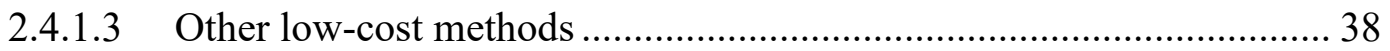

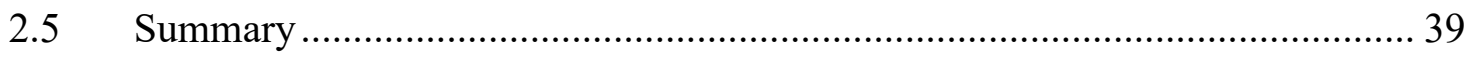

Chapter 3: Ultrasound data acquisition System, phantom design and construction,

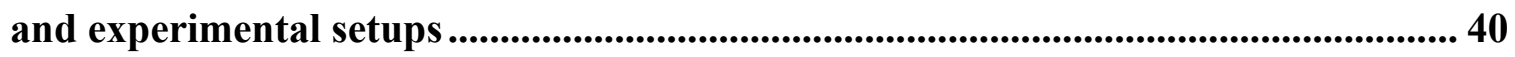

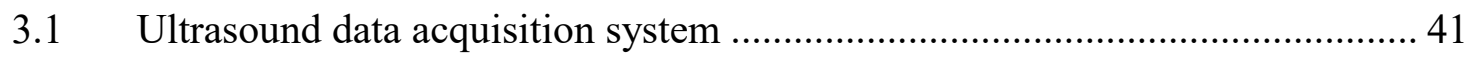

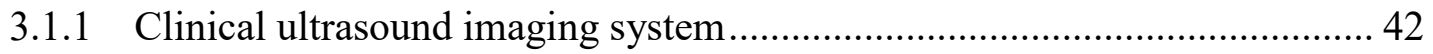




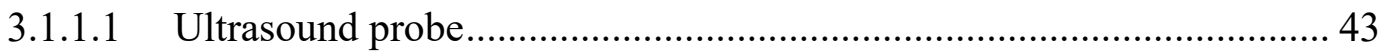

3.1.1.2 Ultrasound Signal specification ....................................................... 45

3.1.1.3 Data Acquisition software and data format ......................................... 45

3.1.2 Wearable ultrasonic sensor (WUS) system................................................. 47

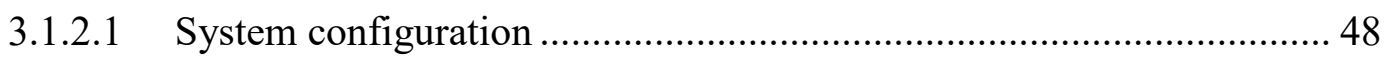

3.1.2.2 Wearable ultrasonic sensor (WUS) .................................................. 50

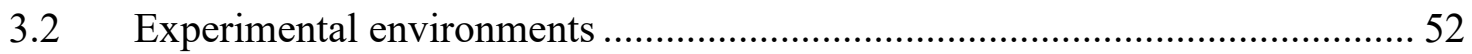

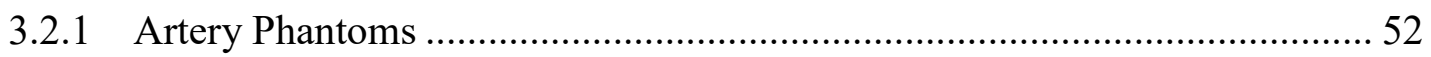

3.2.1.1 Phantom experimental environment ………….................................. 52

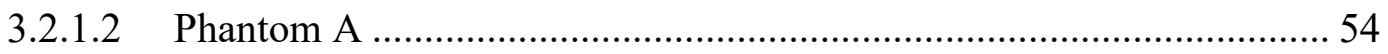

3.2.1.3 Phantom B

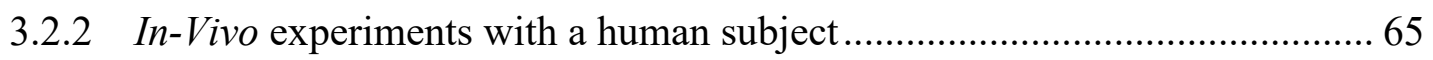

\section{Chapter 4: Algorithm development for artery region detection and lumen diameter}

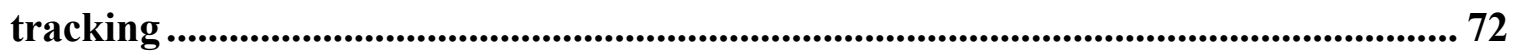

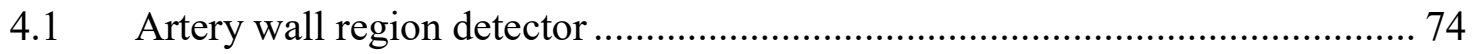

4.1.1 Ground truth creation of the artery wall region by manual segmentation .... 76

4.1.2 Feature Extraction for automatic wall region detection............................... 87

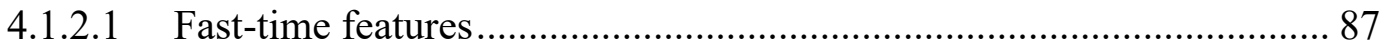

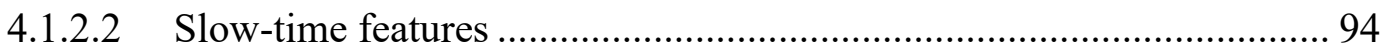

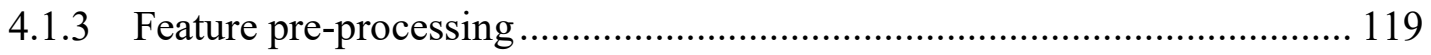

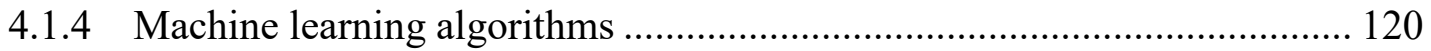

4.1.4.1 Linear Discriminant Analysis (LDA) .............................................. 121

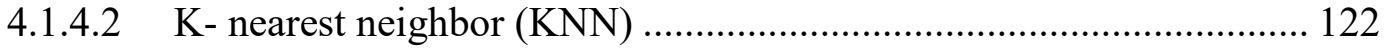


4.1.4.3 Random Forest (RF) ………….................................................. 123

4.1.4.4 Extreme gradient boosting (XGB) ................................................ 124

4.1.4.5 Convolutional neural network (CNN) ............................................... 124

4.2 Artery lumen diameter estimation .......................................................... 130

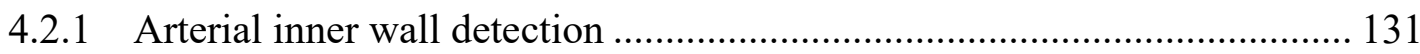

4.2.2 Autocorrelation-based tracking method of lumen diameter change ........... 133

Chapter 5: Results and discussion.................................................................................. 135

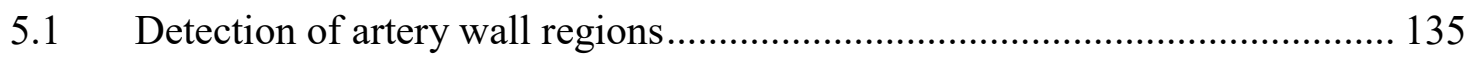

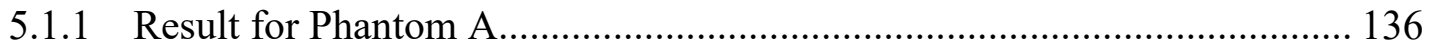

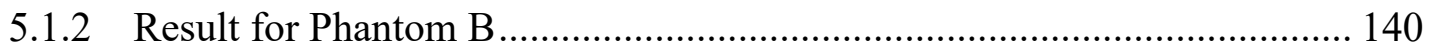

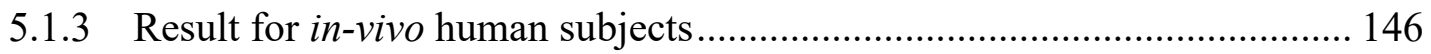

5.1.3.1 PICUS clinical ultrasound imaging system ...................................... 146

5.1.3.2 Wearable ultrasound sensor (WUS) system ..................................... 152

5.2 Tracking of arterial walls and lumen diameter estimation............................. 154

5.2.1 Result for Phantom A........................................................................... 155

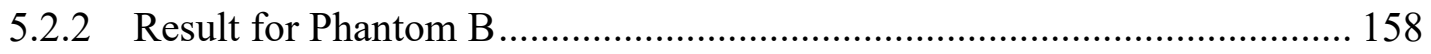

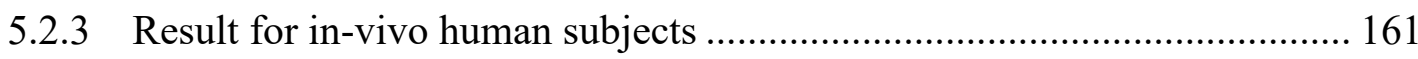

5.2.3.1 PICUS clinical ultrasound imaging system ........................................ 161

5.2.3.2 Wearable ultrasound sensor (WUS) system ..................................... 163

Chapter 6: Conclusion and Future Work.......................................................................... 167

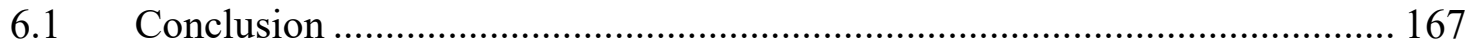

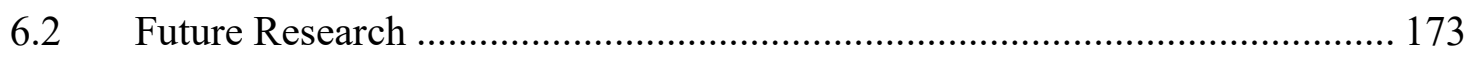




\section{List of Figures}

Figure 2.1 (A) Example of ultrasound rf signal (solid line) and its envelope (A-mode signal) (dashed line), (B) subsection of ultrasound rf signal. 10

Figure 2.2 A-mode signal (lower figure) and measurement configuration of cross-section of the artery in the ultrasound pulse-echo technique (upper figure)................................ 11

Figure 2.3 Illustration of A-mode scan lines, making a B-Mode ultrasound image......... 13

Figure 2.4 B-mode image of the common carotid artery.............................................. 14

Figure 2.5 M-mode image of the common carotid artery.............................................. 15

Figure 2.6 Cross-sectional view of a conduit artery wall. Adapted from [46] ................ 27

Figure 2.7 Schematic of the right common carotid artery, obtained from [52] ............... 29

Figure 2.8 Schematic representation of the Kelvin-Voight model of biological tissue.

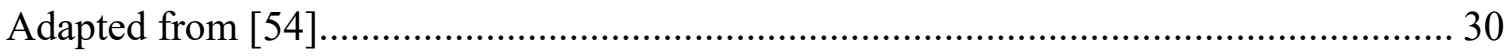

Figure 2.9 An example of stress versus strain curve of materials. ................................... 32

Figure 3.1 Photos of clinical ultrasound imaging system (A) and linear array UT probe (B)

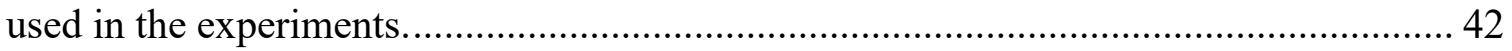

Figure 3.2 Cross-sectional view of the ultrasonic probe. Based on [73] .......................... 44

Figure 3.3 Data Structure of the ultrasound rf signals acquired in B-mode by the PICUS clinical ultrasound imaging system used in the experiments.......................................... 46

Figure 3.4 Block diagram of the WUS system used in the experiments. ........................ 49

Figure 3.5 Photo (A) and schematic (B) of the wearable ultrasonic sensor (WUS) used in

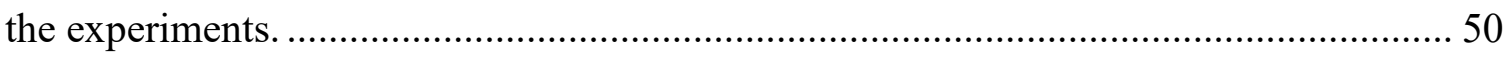


Figure 3.6 Schematic representation of a cross-section of the WUS. Adapted from [73].

Figure 3.7 Schematic view of the phantom experimental environment. ......................... 54

Figure 3.8 Experimental setup for data acquisition with Phantom A........................... 55

Figure 3.9 B-mode image of Phantom A (A) and the corresponding M-mode rf ultrasound

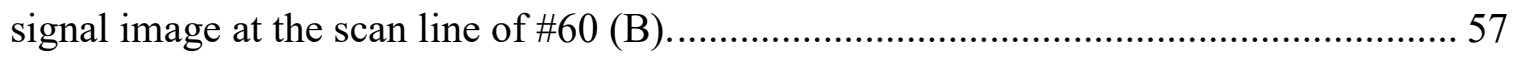

Figure 3.10 A photo of the constructed Phantom B, hydrogel-based tissue-mimicking artery

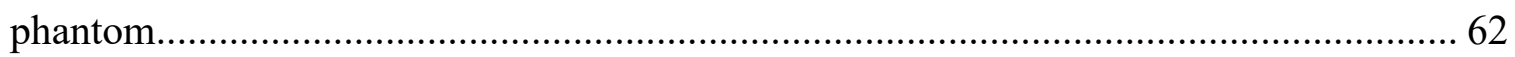

Figure 3.11 Experimental setup for data acquisition of phantom B. ........................... 63

Figure 3.12 B-mode image of Phantom B (A) and the corresponding M-mode image (B)

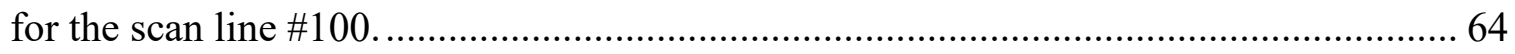

Figure 3.13 Experimental setup for in-vivo data acquisition for the common carotid artery using PICUS clinical ultrasound imaging system with a linear array probe. .................. 66 Figure 3.14 Experimental setup for in-vivo data acquisition for the common carotid artery using WUS. 67

Figure 3.15 B-mode image (A) of in-vivo experiment and the corresponding ultrasound $\mathrm{rf}$ signal M-mode image (B) at the B-mode scan line \#80.

Figure 3.16 M-mode ultrasound rf signal image of the right common carotid artery of a

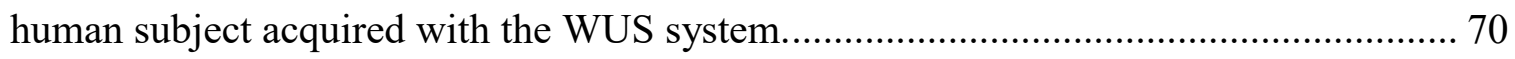

Figure 4.1 Flowchart of artery wall detection and lumen diameter tracking process using M-mode ultrasound rf signal. 73

Figure 4.2 Conceptual sketch of the common carotid artery and expected ultrasound echo peaks indicating the locations of the artery walls. 75 
Figure 4.3 Flowchart of the A-mode scan line segmentation process.

Figure 4.4 Manual selection of the artery wall region in a B -mode signal with water-based

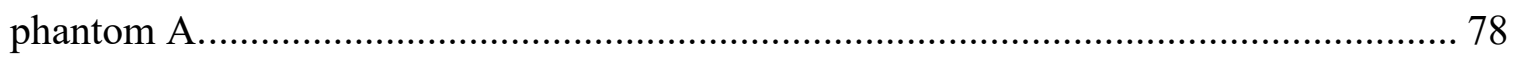

Figure 4.5 Gate selection of artery wall region in the A-mode signal........................... 81

Figure 4.6 Segmented window in an M-mode signal. ............................................ 85

Figure 4.7 Distribution of signal power along fast-time for (A) Phantom A, (B) Phantom

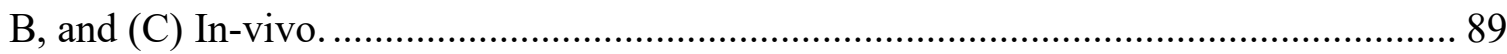

Figure 4.8 Distribution of maximum amplitude along fast-time for (A) Phantom A, (B)

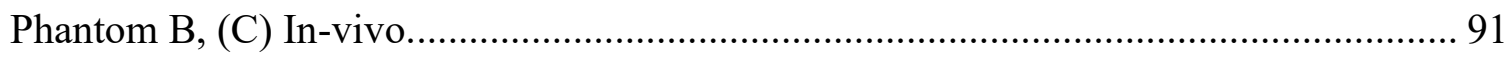

Figure 4.9 Distribution of minimum amplitude along fast-time for (A) Phantom A, (B)

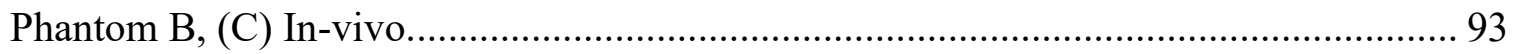

Figure 4.10 M-mode signal and the corresponding maximum amplitude along slow-time

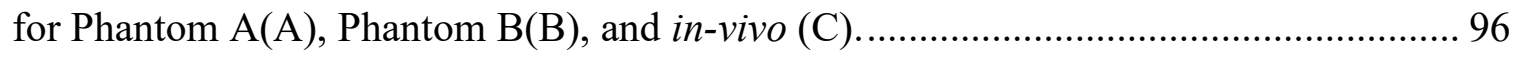

Figure 4.11 Distribution of maximum amplitude values in $\boldsymbol{D R} \mathbf{1}$ along slow-time for (A)

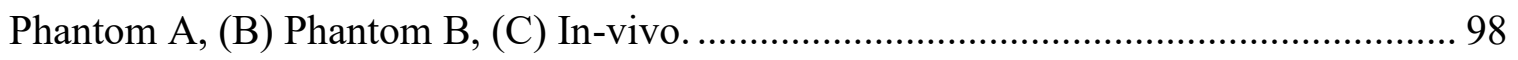

Figure 4.12 Distribution of maximum amplitude values of $\boldsymbol{D R} 2$ along slow-time for (A)

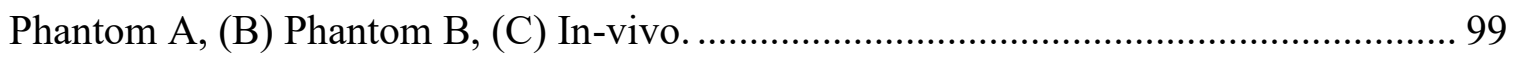

Figure 4.13 Distribution of maximum amplitude values of $\boldsymbol{D} \boldsymbol{R} \mathbf{3}$ along slow-time for (A)

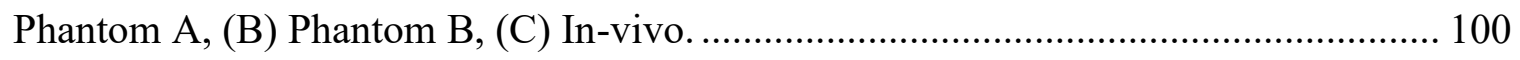

Figure 4.14 Distribution of maximum amplitude values of $\boldsymbol{D R 4}$ along slow-time for (A)

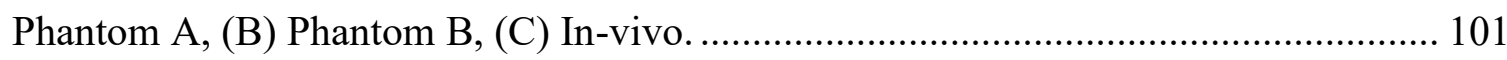

Figure 4.15 Distribution of maximum amplitude values of $\boldsymbol{D} \boldsymbol{R} \mathbf{5}$ along slow-time for (A)

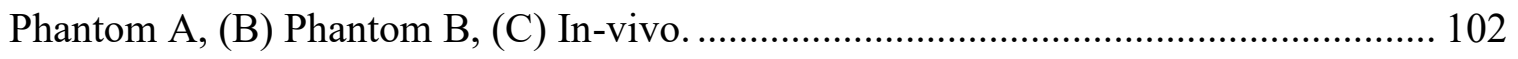


Figure 4.16 M-mode signal and the corresponding minimum amplitude along slow-time for (A) Phantom A, (B) Phantom B, (C) In-vivo. 104

Figure 4.17 Distribution of minimum amplitude values of $\boldsymbol{D R} \mathbf{1}$ values along slow-time for (A) Phantom A, (B) Phantom B, (C) In-vivo. 105

Figure 4.18 Distribution of minimum amplitude values of $\boldsymbol{D} \boldsymbol{R} \mathbf{2}$ along slow-time for (A) Phantom A, (B) Phantom B, (C) In-vivo. 106

Figure 4.19 Distribution of minimum amplitude values of $\boldsymbol{D} \boldsymbol{R} \mathbf{3}$ along slow-time for (A) Phantom A, (B) Phantom B, (C) In-vivo. 107

Figure 4.20 Distribution of minimum amplitude values of $\boldsymbol{D R 4}$ along slow-time for (A) Phantom A, (B) Phantom B, (C) In-vivo. 108

Figure 4.21 Distribution of minimum amplitude values of $\boldsymbol{D} \boldsymbol{R} \mathbf{5}$ along slow-time for (A) Phantom A, (B) Phantom B, (C) In-vivo. 109

Figure 4.22 M-mode signal and the corresponding fractal dimension values along slowtime (A) Phantom A, (B) Phantom B, (C) In-vivo. 112

Figure 4.23 Distribution of fractal dimension value of $\boldsymbol{D R \mathbf { 1 }}$ along slow-time for (A) Phantom A, (B) Phantom B, (C) In-vivo. 114

Figure 4.24 Distribution of fractal dimension value of $\boldsymbol{D R} 2$ along slow-time for (A) Phantom A, (B) Phantom B, (C) In-vivo. 115 Figure 4.25 Distribution of fractal dimension value of $\boldsymbol{D R} 3$ along slow-time for (A) Phantom A, (B) Phantom B, (C) In-vivo. 116 Figure 4.26 Distribution of fractal dimension value of $\boldsymbol{D R 4}$ values along slow-time for (A) Phantom A, (B) Phantom B, (C) In-vivo. 117 
Figure 4.27 Distribution of fractal dimension value of $\boldsymbol{D R} \mathbf{5}$ along slow-time for (A) Phantom A, (B) Phantom B, (C) In-vivo. 118

Figure 4.28 Segmented windows containing (A) proximal wall, (B) distal wall, (C) no boundary for Phantom A obtained with PICUS clinical ultrasound imaging system..... 126 Figure 4.29 Segmented window containing (A) proximal wall, (B) distal wall, (C) no boundary for Phantom B obtained with PICUS clinical ultrasound imaging system..... 127 Figure 4.30 Segmented window containing (A) proximal wall, (B) distal wall, (C) no boundary for in-vivo human subject obtained with PICUS clinical ultrasound imaging system. 128

Figure 4.31 Segmented window containing (A) proximal wall, (B) distal wall, (C) no boundary for human in-vivo subject obtained with Wearable Ultrasound Sensor (WUS) system.

Figure 4.32 Segmented proximal and distal wall regions. 131 Figure 5.1 Normalized confusion matrix of (A) LDA, (B) KNN, (C) RF, (D) XGB and (E) CNN for Phantom A. 138 Figure 5.2 Segmented windows of proximal and distal wall of Phantom A at different instances of time. 139 Figure 5.3 Normalized confusion matrix of (A) LDA, (B) KNN, (C) RF, (D) XGB and (E) CNN for Phantom B. 143

Figure 5.4 Segmented windows of proximal and distal wall of Phantom B at different instances of time. 144

Figure 5.5 Normalized confusion matrix of (A) LDA, (B) KNN, (C) RF, (D) XGB and (E) CNN for in-vivo human subject with PICUS system. 149 
Figure 5.6 Segmented windows of proximal and no boundary of in-vivo human subject with PICUS system at different instances of time.

Figure 5.7 Normalized confusion matrix of CNN for in-vivo human subject with WUS system. 153

Figure 5.8 (A) Displacements of the proximal and distal walls of polymer tube at their corresponding depths, and (B) change of its inner diameter of Phantom A with respect to the measurement time. 155

Figure 5.9 (A) Displacements of the proximal and distal walls of polymer tube at their corresponding depths, and (B) change of its inner diameter of Phantom B with respect to the measurement time. 158

Figure 5.10 (A) Displacements of the proximal and distal walls of carotid artery of human subject at their corresponding depths, and (B) change of its inner diameter with respect to the measurement time. Ultrasound data was acquired with PICUS system. 161 Figure 5.11 (A) Displacements of the proximal and distal walls of carotid artery of human subject at their corresponding depths, and (B) change of its inner diameter with respect to the measurement time. Ultrasound data was acquired with WUS system. 163 


\section{List of Tables}

Table 2.1 Parameters commonly used to diagnose artery healthiness............................. 35

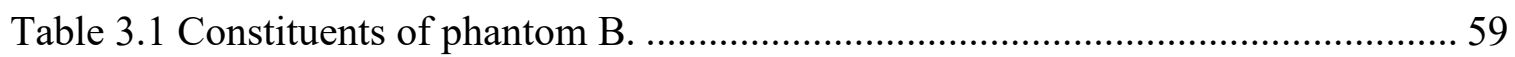

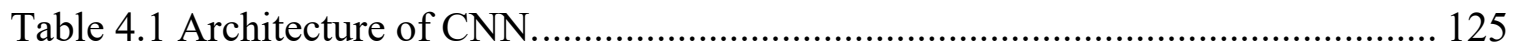

Table 5.1 Accuracy of different machine learning algorithms for Phantom A. ............ 136

Table 5.2 F1 score of different machine learning algorithms for Phantom A. ............... 137

Table 5.3 Accuracy of different machine learning algorithms for Phantom B............... 141

Table 5.4 F1-score of different machine learning algorithms for Phantom B............... 141

Table 5.5 Accuracy of the machine learning algorithms for in-vivo human subject with

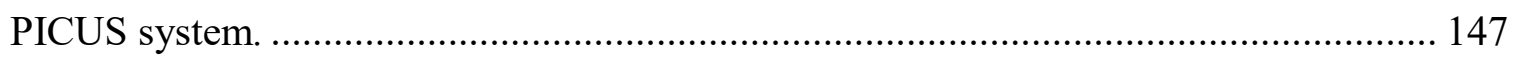

Table 5.6 F1-score of the machine learning algorithms for in-vivo human subject with

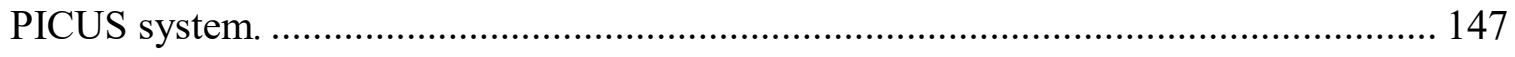

Table 5.7 Accuracy and F1-score of CNN for in-vivo human subject with WUS system.

153

Table 5.8 Minimum and maximum inner diameter of the polymer tube estimated for

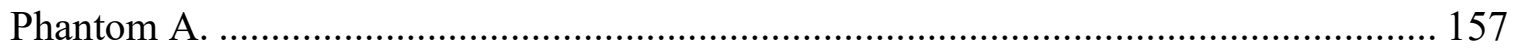

Table 5.9 Minimum and maximum diameter estimated for Phantom B........................ 160

Table 5.10 Diastolic and Systolic diameter estimated for in-vivo experiments.............. 162

Table 5.11 Diastolic and systolic diameter estimated for in-vivo using WUS. .............. 164

Table 5.12 Comparison of mean carotid artery diameters of in-vivo human subject obtained

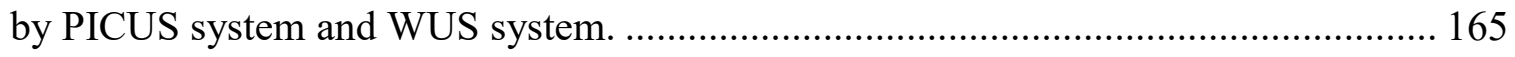




\section{List of Abbreviations}

\begin{tabular}{|c|c|}
\hline A-mode & Amplitude mode \\
\hline ARTSENS & $\begin{array}{c}\text { ARTerial Stiffness Evaluation for } \\
\text { Noninvasive Screening }\end{array}$ \\
\hline B-mode & Brightness mode \\
\hline bps & Beats per second \\
\hline $\mathrm{CNN}$ & Convolution neural network \\
\hline $\mathrm{cST}$ & CentiStokes \\
\hline DTM & Digital thermal monitoring \\
\hline FD & Fractal dimension \\
\hline FMD & Flow-mediated dilatation \\
\hline fMRI & Functional magnetic resonance imaging \\
\hline KNN & K-nearest neighbor \\
\hline LDA & Linear discriminant analysis \\
\hline M-mode & Motion mode \\
\hline MSE & Mean squared error \\
\hline PVDF & Polyvinylidene difluoride \\
\hline PZT & Lead zirconate titanate \\
\hline $\mathrm{RF}$ & Random forest \\
\hline rf signals & Radio frequency signals \\
\hline
\end{tabular}




\begin{tabular}{|c|c|}
\hline SMOTE & $\begin{array}{c}\text { Synthetic minority oversampling } \\
\text { technique }\end{array}$ \\
\hline SNR & Signal-to-noise-ratio \\
\hline UT & Ultrasonic transducer \\
\hline WUS & Wearable ultrasound sensor \\
\hline XGB & Extreme gradient boosting \\
\hline
\end{tabular}




\section{List of Symbols}

\begin{tabular}{|c|c|c|}
\hline Symbols & Definition & Units \\
\hline$d_{s}$ & Systolic artery lumen diameter & $\mathrm{mm}$ \\
\hline$d_{d}$ & Diastolic artery lumen diameter & $\mathrm{mm}$ \\
\hline$p_{s}$ & Systolic blood pressure & $\mathrm{mmHg}$ \\
\hline$p_{d}$ & Diastolic blood pressure & $\mathrm{mmHg}$ \\
\hline$x[i]$ & Signal & \\
\hline$e[i]$ & Envelope of signal $x[i]$ & \\
\hline $\operatorname{DHT}(x[i])$ & $\begin{array}{l}\text { Discrete Hilbert Transform of } \\
\qquad x[i]\end{array}$ & \\
\hline$\tau_{p w}$ & Shift in proximal wall & $\mathrm{mm}$ \\
\hline$\tau_{d w}$ & Shift in distal wall & $\mathrm{mm}$ \\
\hline$r$ & Pearson correlation coefficient & \\
\hline$P_{\text {inner }}$ & Location of proximal inner wall & $\mathrm{mm}$ \\
\hline$D_{\text {inner }}$ & Location of distal inner wall & $\mathrm{mm}$ \\
\hline$\Delta D(t)$ & Lumen diameter of artery wall & $\mathrm{mm}$ \\
\hline$P W_{t}[i]$ & $\begin{array}{l}\text { Signal with proximal wall at } \\
\text { instance of time } t\end{array}$ & \\
\hline$D W_{t}[i]$ & $\begin{array}{c}\text { Signal with distal wall at instance } \\
\text { of time } t\end{array}$ & \\
\hline
\end{tabular}




\section{Chapter 1: Introduction}

In this chapter, the thesis overview is provided along with its objectives. Also, thesis contribution and organization are outlined.

\subsection{Overview}

Cardiovascular diseases are estimated to be responsible for $31 \%$ of deaths worldwide [1]. Coronary artery disease, a type of cardiovascular health disease, was the leading cause (43.2\%) of deaths attributable to cardiovascular disease [2]-[4]. The coronary artery disease is complicated to treat at advanced stages but can be cured by just switching to a healthier lifestyle if identified at earlier stages. Further, detecting coronary artery disease at the earlier stages would bring down the direct and indirect spending to treat cardiovascular diseases which was 329.7 billion dollars worldwide, in the year 2013-14 [2]. Several kinds of research indicate that the ultrasonic monitoring of the artery stiffness has proved beneficial in detecting coronary artery disease at an early stage. Various parameters that describe the condition of the artery wall such as its distensibility, stiffness, and compliance, have been strongly associated with cardiovascular diseases [5]-[9]. Therefore, these parameters may be used as predictors for cardiovascular health risks. The artery compliance for the common carotid artery help in the early detection of coronary heart disease [10], a type of cardiovascular disease. The coronary artery disease is also called as a "sneaky disease"[11], owing to its difficulty to be identified at the early stages, but it could be cured just by modifying the lifestyle when it is nascent [12]. Mass screening 
people for such a disease by checking the above-mentioned arterial parameters at regular intervals helps in early intervention. Therefore, for the prevention and diagnosis of coronary heart disease at early stages, a cost-effective, and efficient technique is required for the mass screening of patients for the parameters indicating the stiffness of the artery. All the parameters mentioned above, indicating the artery stiffness can be estimated noninvasively by measuring the change in the blood pressure, artery wall thickness, and/or artery diameter. There are many innovative and cost-efficient methods available to measure the change in pressure and diameter of the artery using ubiquitous devices. For instance, blood pressure can be monitored using the pulse transit time [13], [14], either with a pressure transducer in a smartphone [15], or optical imaging technology [16], [17]. Examples of the methods to detect the change in the diameter of the artery are brightness mode (B-mode) image of ultrasound artery diameter detection [18], [19], motion mode (Mmode) ultrasound using a single ultrasound transducer (UT) [20]-[22], and digital thermal monitoring [23]. After assessing the different techniques presented to measure the change in the diameter of the artery, it is observed that the use of a single UT to measure the change in the diameter could be efficient and effective for mass screening purposes. The main advantages of using M-mode signal over B-mode signals to estimate the artery lumen diameter is that it can be obtained using a single UT. The advantages of using a single UT are: (i) The lower cost associated with the ultrasound transducer when compared to a Bmode ultrasound transducer (array of single UT); (ii) lower power requirement when compared to the B-mode ultrasound imaging system. These two advantages encourage the development of flexible Wearable ultrasound sensor (WUS) system [24], [25]. The two main challenges of using M-mode ultrasound signal from a single UT are the following: 
(1) identifying the artery location for ultrasound signal acquisition without any B-mode image guidance, (2) manually identifying the wall echoes in the ultrasound radio-frequency (rf) signals, to detect and track the artery walls for measuring the change in the diameter. The motivation behind the research presented in this thesis is to develop an automatic artery detection, and diameter estimation technique using ultrasound radio-frequency (rf) signals

obtained by cost-effective UTs in M-mode, but not brightness mode (B-mode) imaging [26]-[28].

\subsection{Problem statement}

Regular evaluation of the artery parameters such as stiffness, compliance, and distensibility, which are based on the measurement of the change in pressure and diameter, helps in the prevention and diagnosis of coronary heart disease [12]. A significant amount of financial and technical resources is needed to perform the mass-screening test for the above-mentioned arterial parameters regularly. In recent years, new cost-effective and non-invasive techniques, as mentioned in Section 1.1, have been developed to measure the change in the blood pressure or artery diameter. The existing algorithms for diameter estimation using ultrasound rf signal with single UT as specified in [20]-[22] make prior assumptions regarding the diameter and thickness of the artery and require manual localization of approximate region in which the artery wall is located to be manually specified by the user. Prior information about the artery, whose diameter is to be measured, should be identified before the measurement process. This prior assumption regarding the diameter and thickness of the artery should be generic, and such take into account the factors such as age, ethnic background, height, weight, and gender, which influence the 
diameter and thickness of the artery. Due to all these factors, it is challenging to develop a generalized for the measurement of diameter for different arteries of interest. As the approximate region of depth in which the artery wall is to be found should be manually specified, it slows down the process of screening each patient. Further, the operator specifying the region containing the artery wall should be trained to differentiate between the echoes of stationary tissue and moving artery walls.

Also, there are several challenges in the current methods to measure the change in the artery diameter using a single UT. The pressue applied by the UT during the data acquisition causes deformation of the artery and introduces measurement errors. Movement of either the patient or the UT during data acquisition introduces motion artifacts. Medical professional is needed to perform data acquisition. Since single UT (conventional single UT or WUS) uses a plane wave unlike the focused ultrasound in clinical ultrasound imaging system, there are more scattering noises in ultrasound M-mode data acquired. The higher scattering noises reduces the signal-to-noise ratio (SNR) of the artery wall boundary echoes.

Currently, there is no wearable sensor system for continuously monitoring the change in the artery diameter. Thus, there is a need for a lightweight and flexible wearable film ultrasound sensor along with an automatic artery diameter detection technique. The automatic artery diameter detection technique could eliminate the need for an experienced operator to locate the region of artery walls manually. It could prove to be more beneficial in developing a self-contained (no operator required) system for artery diameter 
measurement. Unlike the conventional UT which are made of piezoelectric ceramic material, such as lead zirconate titanate (PZT), a wearable ultrasonic sensor (WUS) is made of piezoelectric polymer material, such as polyvinylidene difluoride (PVDF), and may improve the accuracy in the measurement of artery diameter by reducing the motion artifact and deformation of the artery. It is to be noted that unlike conventional UTs, WUS does not apply any external pressure on the artery. Also, it does not need an operator to hold the UT at a fixed position during the data acquisition. A WUS [24]-[26], [28] can be glued or taped on to the region of interest for ultrasound data acquisitions. Therefore, there is no relative motion between the sensor and the subject which reduces the motion artifact.

Further, a WUS could be used for continuous and long-term monitoring of the artery parameters at home. Due to the advantages of the WUS over the conventional UTs, an automatic artery detection technique designed for a low-cost WUS system could be a promising candidate in making the mass screening test of people, for coronary artery disease. This research is aiming at developing and evaluating an automatic artery detection and diameter tracking algorithm for a WUS system.

\subsection{Objectives}

The primary goal of this thesis is to develop an automatic artery detection and lumen diameter tracking algorithm using M-mode rf ultrasound signals acquired with a single UT. The thesis research involves: a detailed literature review of the existing artery diameter measurement technique; development of a machine learning-based automatic artery region detection and correlation-based lumen diameter measurement technique; design and 
construction of artery mimicking phantoms to acquire ultrasound data; test and evaluation of the developed algorithms in both phantom and in-vivo experiments using the PICUS clinical ultrasound imaging system. Finally, the proposed algorithms are tested with invivo ultrasound data obtained by the WUS system.

\subsection{Thesis Contributions}

The following are the main contributions presented in the thesis:

- Phantom experimental environments are designed and constructed using a polymeric tube and a soft tissue-mimicking phantom that simulates the cardiac cycle of the artery to test and evaluate the proposed algorithms.

- Machine learning-based artery region detection techniques are developed and implemented on M-mode ultrasound rf signals. A set of machine learning-based detection techniques such as Linear discriminant analysis (LDA), K-nearest neighbor $(\mathrm{KNN})$, Random forest (RF), Extreme gradient boosting (XGB) and Convolution neural network $(\mathrm{CNN})$ are compared using both manually and automatically extracted features. A performance evaluation of the developed techniques is carried out with the ultrasonic data obtained by the PICUS clinical ultrasound imaging system from the artery phantom experimental environments and human subjects.

- A correlation-based automatic lumen diameter tracking technique is developed to locate the artery inner walls and track the artery lumen diameter automatically during the cardiac cycle. The developed techniques are tested and compared using the ultrasonic data acquired by the PICUS clinical ultrasound imaging system on phantom experimental environments and human subjects. 
- The developed algorithm is tested on the ultrasound signal obtained from the human subjects with a flexible ultrasound sensor system. The CNN classifier identified the artery wall region with an accuracy of $88.9 \%$ and $88.3 \%$ on data acquired by a PICUS clinical ultrasound acquisition system and WUS system.

\subsection{Thesis Organization}

Chapter 1 presents the background and objectives of this thesis research.

Chapter 2 provides an overview of the artery characteristics, technical review of machine learning algorithms, and a detailed literature review of the existing technologies to measure the artery diameter. This chapter also provides a general technical background on the ultrasound measurement principle.

Chapter 3 describes the design and construction of two phantom experimental environments (phantom A and phantom B) to mimic the artery and surrounding tissues for testing the proposed algorithms of automatic artery detection and diameter estimation. This chapter also includes experimental configurations and two different ultrasound acquisition systems (clinical ultrasound imaging system and WUS system) used in the experiments, the experimental setup and the data format of the acquired ultrasound signal.

Chapter 4 describes the overview of the proposed algorithm for automatic artery detection and lumen diameter tracking using ultrasound signals obtain in motion mode (M-mode), ground truth extraction to train and evaluate the algorithm, feature selection process and 
explains the different algorithms used for artery boundary detection and motion tracking to estimate artery diameter and its changes.

Chapter 5 provides a performance comparison of proposed algorithms with data acquired from phantom A, phantom B, and human subjects using two ultrasound data acquisition systems (PICUS clinical ultrasound imaging system and WUS system). It also discusses the results of the correlation-based lumen diameter tracking method for all the experimental configurations.

Chapter $\mathbf{6}$ concludes the thesis by summarizing the obtained result outcomes and provides recommended future work for further improvement. 


\section{Chapter 2: Background Review}

\subsection{Technical basis}

This section serves as an outline of medical ultrasound imaging, the machine learning algorithms used in this study, the anatomy of the common carotid artery, and the current technologies used to diagnose artery.

\subsubsection{Ultrasound Imaging}

Ultrasound imaging is regularly used in medical imaging owing to its inherent characteristics of non-invasiveness and no ionization radiation [29]. From prior knowledge of acoustic wave propagation velocity through the medium of interest and measurement of the propagation time of reception of the echoes from a reflector of interest, the distance from the ultrasound probe to the reflection source can be determined [29]. The distance information alongside the amplitude of the received echo is used to form the ultrasound image. Any sound signal that is above the human audible frequency range (20 to 20,000 $\mathrm{Hz}$ ) is called as ultrasound signal. Since, the frequency range of the ultrasound is also within the radio signal frequency range ( $3 \mathrm{kHz}$ to $300 \mathrm{GHz}$ ), it is also called as ultrasound radio-frequency signals or ultrasound rf-signals. In ultrasound imaging, the envelope of the ultrasound rf-signal as shown in Figure 2.1 is usually referred to as ultrasound signal. The ultrasound propagation path of the received echo is referred to as a scan line (see Figure 2.3). Therefore, an ultrasound radio-frequency (rf) signal is composed of the echoes from reflection sources on the scan line. The solid line in Figure 2.1 presents an example of an ultrasound rf signal acquired. 
(A)
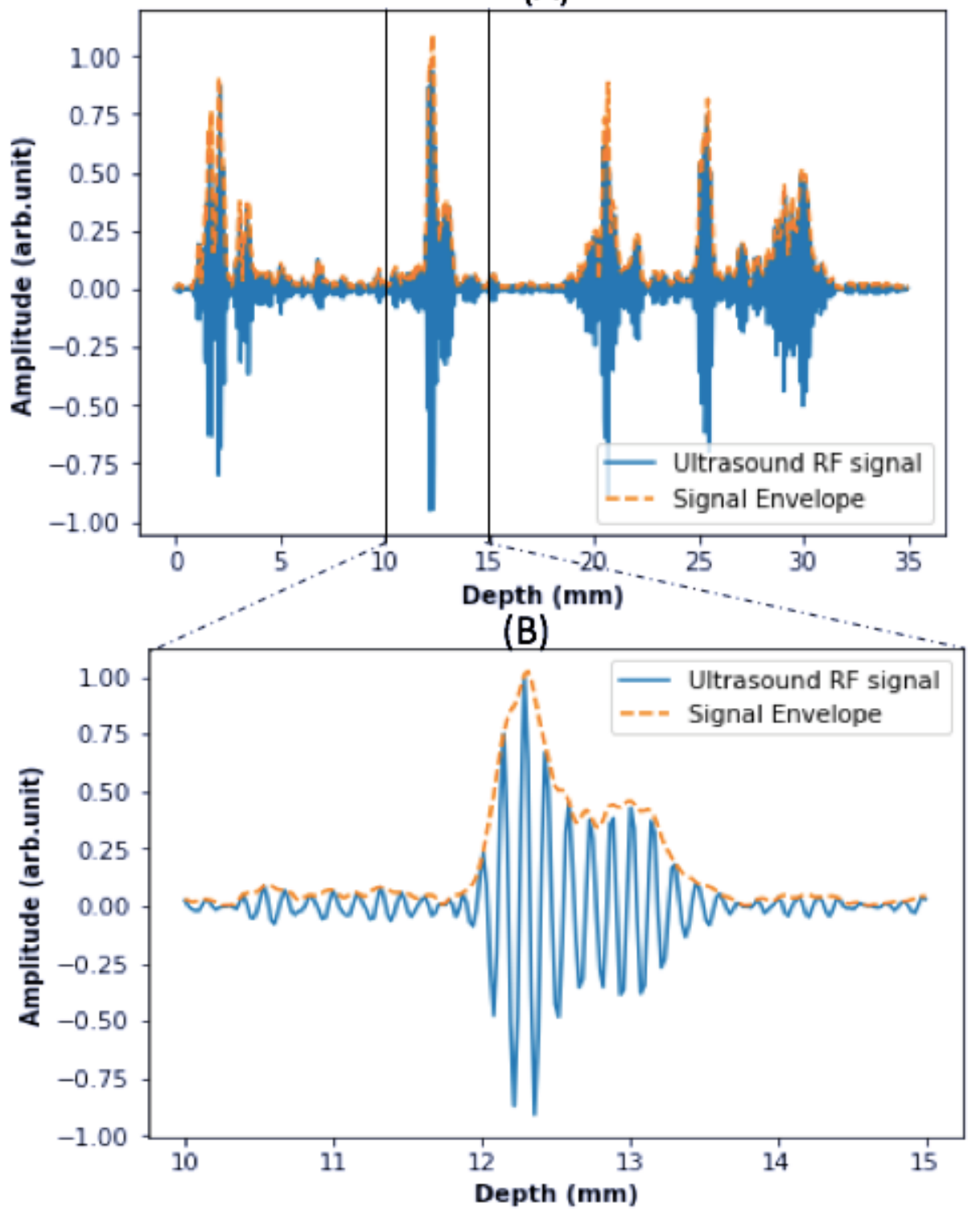

Figure 2.1 (A) Example of ultrasound rf signal (solid line) and its envelope (A-mode signal) (dashed line), (B) subsection of ultrasound rf signal. 
The envelope of this ultrasound rf signal is called the A-mode (amplitude mode) signal or ultrasound signal, is the most basic ultrasound signal that can be used to construct an ultrasound image. Different ultrasound imaging are constructed from these A-mode signals. Two imaging modes used in this thesis are M-mode (motion-mode) and B-mode (brightness-mode). These three modes are explained in detail as follows.

\subsubsection{A-Mode}

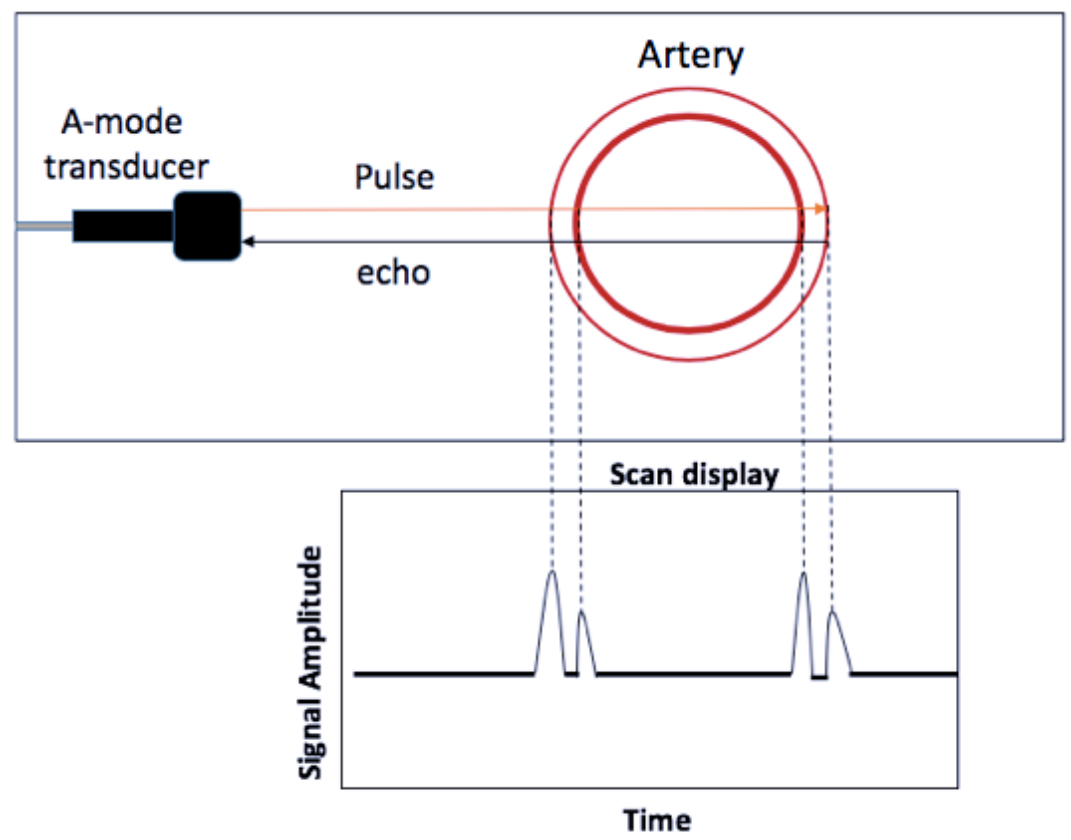

Figure 2.2 A-mode signal (lower figure) and measurement configuration of cross-section of the artery in the ultrasound pulseecho technique (upper figure).

Figure 2.2 illustrates the operation of an A-mode acquisition and the generated scan line in the ultrasonic pulse-echo technique. In the pulse-echo technique, the pulsed ultrasound is 
generated, transmitted, and received by an ultrasonic transducer (UT), which acts as both a transmitter and a receiver. A-Mode signal is obtained from the envelope of the received ultrasound rf signal, as shown by the dotted line in Figure 2.1. A-mode signal maps the intensity of received echoes along the tissue depth direction (ultrasound propagation path). As shown in Figure 2.1, the changes in the received echo amplitude occur some of the transmitted ultrasound waves are reflected from the boundaries occurs because of the difference in the acoustic impedance of the media before and after the boundary and are called as boundary echoes. To correctly identify the boundary location of the artery or any organ of interest, the approximate depth at which they are located should be known. As the rf echoes are composed of both tissue boundary echoes and speckle noises, it is difficult to identify the location of tissue just with the help of the A-mode signal. Thus some prior knowledge, such as the approximate region of depth or characteristics of the tissue boundary of interest, should be known to make a meaningful decision about the location of the tissue or organ boundary of interest from the A-mode signal [29]. 


\subsubsection{B-Mode}

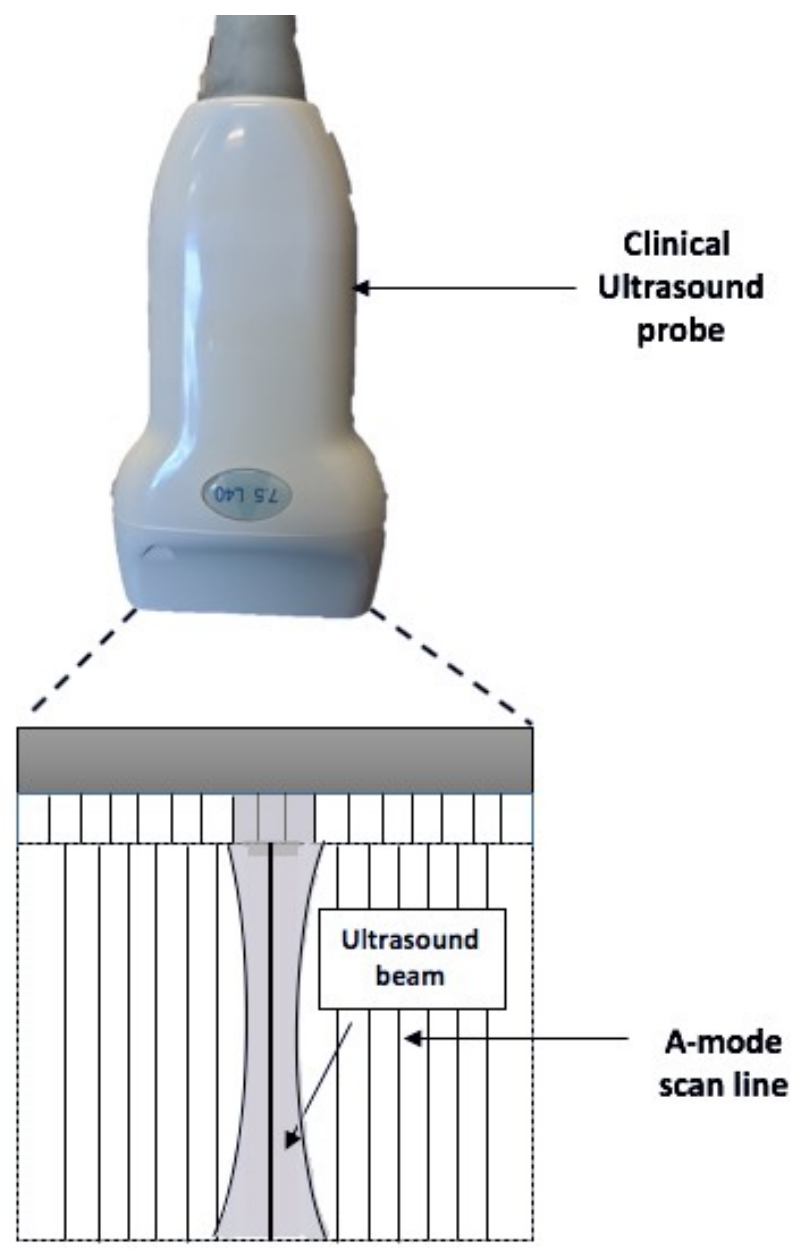

Figure 2.3 Illustration of A-mode scan lines, making a B-Mode ultrasound image.

A B-mode scan is used to obtain a two-dimensional ultrasound image of the cross-section of the tissues in which the UT array probe is placed, or a single UT is moved across the body surface. The ultrasound probe for B-mode used in this thesis is a linear UT array probe. The B-mode image is constructed from a series of A-mode signals, as shown in Figure 2.3. 


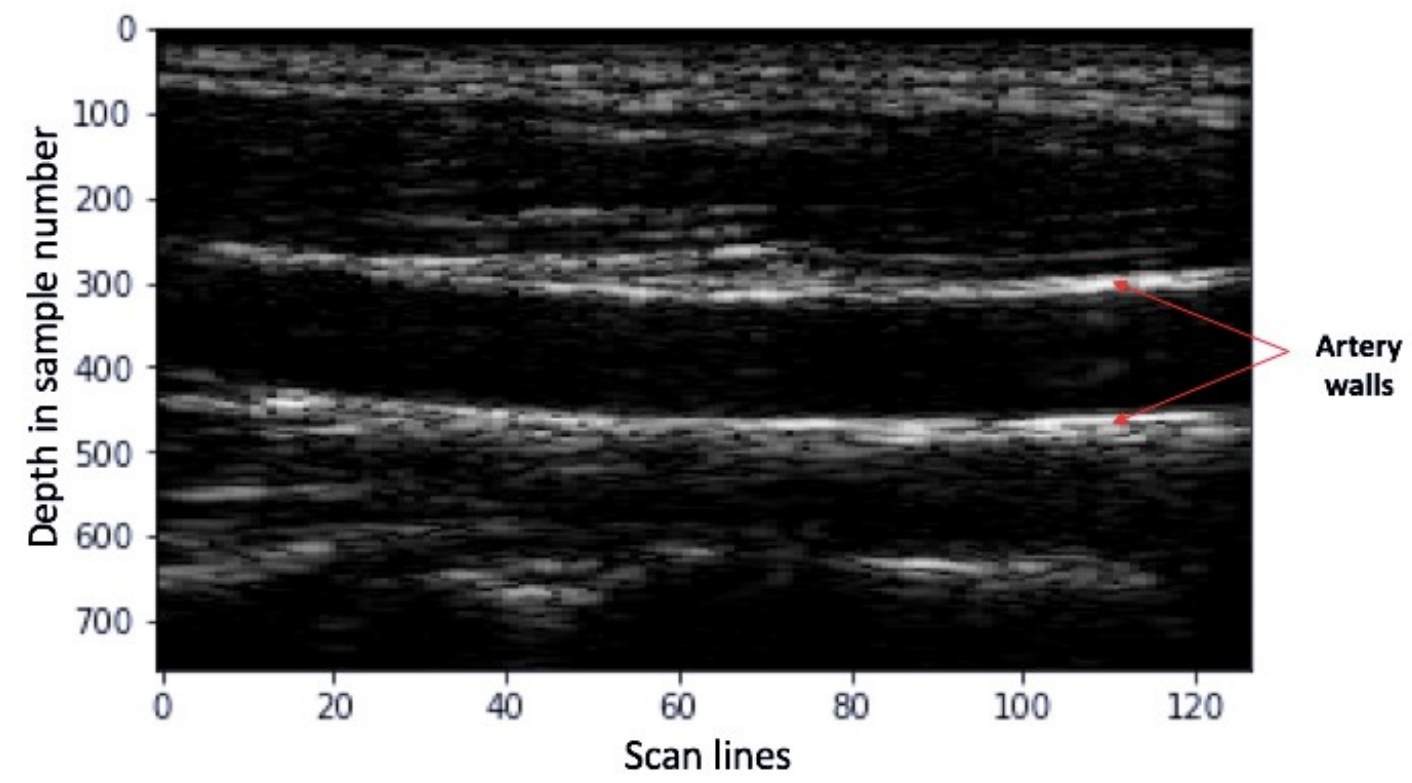

Figure 2.4 B-mode image of the common carotid artery.

An example of a B-Mode image taken over the common carotid artery is shown in Figure 2.4. B-Mode is useful in diagnostic and observational purposes as it allows physicians and researchers to observe internal $2 \mathrm{D}$ structures in real-time non-invasively. B-mode imaging is also commonly used to aid the cannulation of arteries with intravascular catheters and needles. The main advantage of using B-mode for artery diameter tracking is that the PICUS clinical ultrasound imaging probe can be precisely positioned over the artery for diameter measurement with the help of a B-mode image. In case of using a single UT to track the diameter of the artery, it is difficult to position the transducer over the artery without any visual feedback. This advantage of the B-mode based diameter tracking comes 
at the cost of hardware and software of an ultrasound scanner, which increases up the total cost of the acquisition system when compared to a single UT system [29].

\subsubsection{M-Mode}

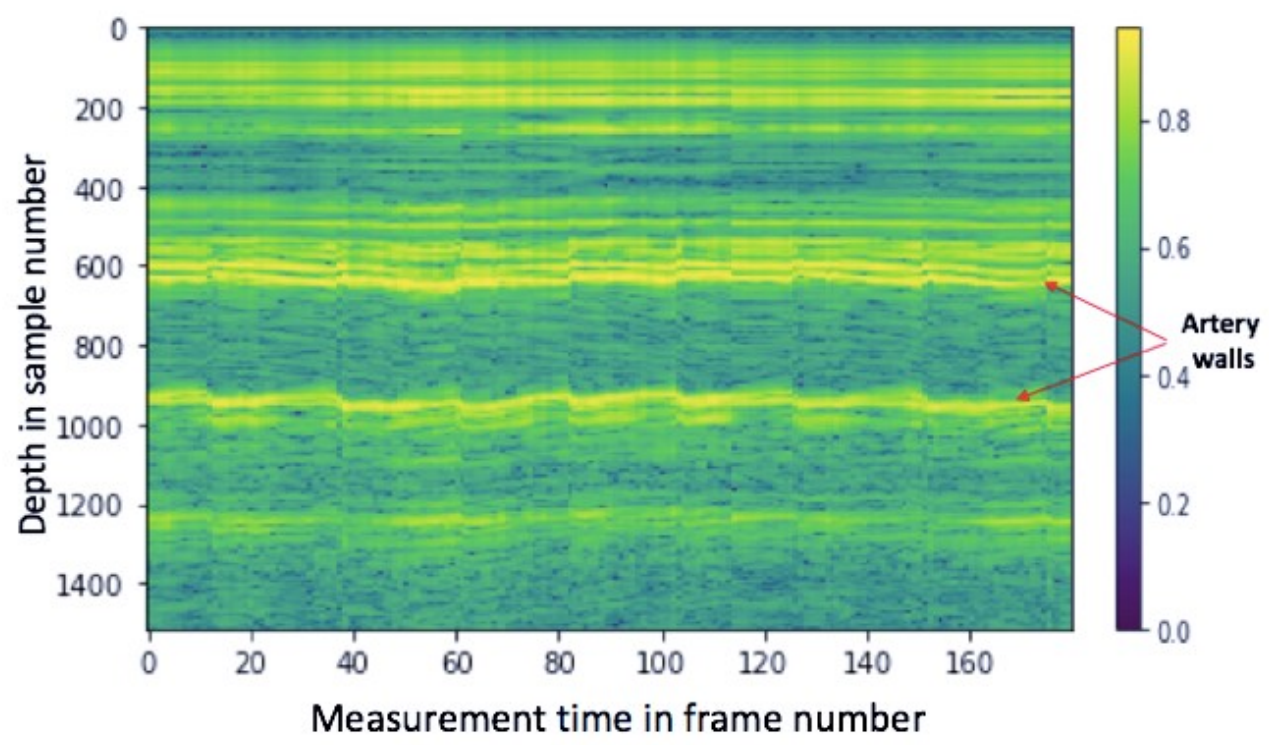

Figure 2.5 M-mode image of the common carotid artery.

M-mode imaging is used to visualize and capture the tissue motion by capturing the Amode signal amplitude changes at chosen location over time. M-mode imaging can be obtained by an array UT probe using only the selected UTs at a chosen location is used. M-mode image can also be obtained using a single UT at the chosen location. An example of an M-mode image of the common carotid artery is shown in Figure 2.5. The change in the diameter of the carotid artery can be observed in the image. The artery wall that is closer to the ultrasound probe is called as the proximal wall and the far wall is referred as the distal wall henceforth. The proximal and the distal wall of the carotid artery is found to 
be moving opposite to each other due to the blood pressure in the arterial wall. M-mode imaging provides valuable information on tissue structures, such as arteries and valves of the heart, which undergo some form of motion. B-mode image often is used to position the M-mode scan line over the area of interest. This dual form of image generation allows for an ultrasound operator to easily position the M-mode element with the help of the B-mode cross-sectional image [29]. Since the M-mode image can be obtained by a single UT and provides information about the motion of tissues, it can be used to track the artery diameter. The benefit of using M-mode to track the change in artery diameter over the B-mode imaging technique is that it requires less hardware of ultrasound electronics (as it can be acquired using a single UT) and less computation for signal processing. It is because the B-mode acquisition over a period of time produces a series of images which are to be processed to obtain the change in the diameter of the artery. While, in the case of M-mode imaging, the diameter change of the artery over a period of time can be measured by just processing a single M-mode image.

\subsubsection{Review of machine learning methods used in this study}

The following section contains the technical review of the machine learning methods that are used in this thesis. Since no single algorithm works best for all conditions, different machine learning techniques are selected to evaluate the performance of the features, explained in Section 4.1.2. The algorithms for comparison are chosen, such that it includes a parametric model, a non-parametric model, meta-learning tree-based models, and a neural network-based model. 


\subsubsection{Linear Discriminant Analysis (LDA)}

The linear discriminant analysis classifiers are parametric classification algorithms that model class densities as multivariate Gaussian distributions[30]. The probability density function of class $c$ in $R^{p}$ is given by Equation 2.1 .

$$
f_{c}(x)=\frac{1}{\sqrt{(2 \pi)^{p}\left|\sum_{c}\right|}} e^{-\frac{1}{2}\left(x-\mu_{c}\right)^{T} \sum_{c}^{-1}\left(x-\mu_{c}\right)}
$$

where $\mu_{c}$ and $\sum_{c}$ are the class-specific mean and covariance matrix, respectively. $\left|\sum_{c}\right|$ is the determinant of the covariance matrix. Given that the prior of class $c$, that is the probability that a random sample belongs to class $c$, is $\pi_{c}$. The posterior probability can be obtained such that a sample $X=x$ belongs to class $c$ by applying Bayes theorem. Since $G$ is discrete, this posterior probability is given by Equation 2.2.

$$
P(G=c \mid X=x)=\frac{f_{c}(x) \pi_{c}}{\sum_{l=1}^{C} f_{1}(x) \pi_{l}}
$$

The learning of a classifier is done by maximizing the posterior probability over the class labels, given by Equation 2.3.

$$
\hat{G}=\arg \max _{c} P(G=c \mid X=x)
$$


where $\hat{G}$ is the predicted class label [31]. The primary assumption in linear discriminant analysis is that all classes have the same covariance matrix, $\forall \mathrm{c}: \sum_{c}=\sum$, and a pooled estimate is used for all classes. The linear discriminant analysis is implemented with the help of the Sklearn library provided by [32]. The main drawback of LDA is that it assumes that all the features is Gaussian distribution by default. However, the features that are extracted, as explained in Section 4.1.2, are not always Gaussian necessarily. Thus, a non-parametric method that does not make any assumptions regarding the distribution of the features is explained in the next section.

\subsubsection{K-Nearest Neighbor (KNN)}

The K-nearest neighbor algorithm is a simple non-parametric distance-based algorithm. Predictions are made for a new instance $\left(x_{t}\right)$ by searching through the entire training set for the $\mathrm{K}$ most similar instances (the neighbors) and summarizing the output variable for those $\mathrm{K}$ instances [33]. Nearest neighbour is a learning-by-memorization type of rule which requires the entire training data set to be stored and scanned to find its neighbors at test time. The memorization type rule leads to expensive computation at test time [33]. For a given instance $x_{t}$

$$
y_{t}=\arg \max _{c \in\left\{c_{1}, c_{2}, \ldots, c_{m}\right\}} \sum_{x_{i} \in N\left(x_{t}, k\right)} E\left(y_{i}, c\right)
$$

where the functions $E$ and $N$ are given by Equation 2.5

$$
E(a, b)=\left\{\begin{array}{lr}
1 & \text { if } a=b \\
0 & \text { else }
\end{array}\right.
$$




$$
N(x, k)=\text { Set of } K \text { nearest neighbor of } x
$$

where $y_{t}$ is the predicted class for the query instance $x_{t}$ and $m$ is the number of classes present in the data. The set of the $K$ nearest neighbor is calculated by measuring the distance between the given instance, and the neighboring points considered [33].

List of parameters to be chosen in the algorithm are given as follows [32].

- N neighbors: The number of neighbors or the value of $k$ is specified in this parameter.

- Weights: The weight that is assigned to the neighboring points that are considered.

- Distance metric: The distance that is used to measure the nearest point for a given instance. The distance metric such as Minkowski, Manhattan, or Euclidean.

The distance-based, non-parametric method might perform better than the LDA if the features considered are non-parametric, but the main drawback is that the computational complexity of KNN is higher than LDA. The higher computational complexity is because, unlike most of the algorithms, the KNN does not learn anything from the training data and uses the training data itself for classification.

\subsubsection{Random Forest (RF)}

Random forest is a type of ensemble machine learning algorithm called bootstrap aggregation or bagging. An ensemble method is a technique that combines the predictions from multiple machine learning algorithms together to make more accurate predictions than any individual model [34]. The idea in bagging is to average many noisy but approximately unbiased models, and hence reduce the variance [35]. The decision trees 
[36] are ideal for the case of bagging as they are non-parametric models, which capture complex interaction. The main drawback of the decision trees is that there is a very high variance observed in the model depending on the set of the input data to the model. The problem of overfitting the training data observed in the case of the decision trees, when the trees are grown deep (e.g., few training samples at each leaf-node of the tree) and the trees are not pruned is rectified with the help of bagging [37].

Random forests are an improvement over bagged decision trees that rectify the problem of the bagged decision trees. Since the decision tree uses a greedy algorithm to minimize error, even with Bagging, the decision trees can have a lot of structural similarities and in turn, have a high correlation in their predictions. The combined predictions of the multiple models work better if the predictions from the sub-models are uncorrelated [38]. Hence, the random forest changes the algorithm for the way that the sub-trees are learned so that the resulting predictions from all of the subtrees have less correlation. In the case of the decision trees, the learning algorithm is allowed to look through all variables to select the most optimal split-point. The random forest algorithm changes this procedure so that the learning algorithm is limited to a random sample of features of which to search [35]. The parameter that is to be selected when using a random forest machine learning algorithm is the number of samples, number of trees to include, and the number of split points for every tree considered [36]. Compared to KNN, random forests are much harder to construct and requires more time to construct. Since random forests are a combination of multiple decision trees, it has a very high computational complexity. However, the random forests overcomes the problem of overfitting by averaging or combining the results of different 
decision trees. Further, they work correctly for a broad range of data items than a single decision tree. The algorithm for the random forest is given below.

\author{
Algorithm 2.1 \\ For $b=1$ to $B$ : \\ (a) Draw a bootstrap sample $Z^{*}$ of size $N$ from the training data. \\ (b) Grow a random-forest tree $T_{b}$ to the bootstrapped data, by re- cursively repeating the \\ following steps for each terminal node of the tree, until the minimum node size $n_{\min }$ is \\ reached. \\ i. Select $m$ variables at random from the $p$ variables. \\ ii. Pick the best variable/split-point among the $m$. \\ iii. Split the node into two daughter nodes. \\ Output the ensemble of trees $\left\{\mathrm{T}_{b}\right\}_{1}^{\mathrm{B}}$. \\ To make a prediction at a new point $x$ : \\ Classification: Let $C_{b}^{\wedge}(x)$ be the class prediction of the $b_{t h}$ random forest tree. \\ Then $C_{r f}^{\wedge}(x)=$ majority vote $\left\{C_{b}^{\wedge}(x)\right\}_{1}^{B}$.
}

\title{
2.1.2.4 Extreme Gradient Boosting (XGB)
}

The extreme gradient boosting is a type of ensemble learning that uses the gradient boosting on the decision trees proposed by Friedman [34]. Boosting is an ensemble technique where new models are added to correct the errors made by existing models. Gradient boosting is an approach where new models are created to predict the residuals or errors of prior models and then added together to make the final prediction [39]. The gradient descent algorithm [40], is used to minimize the loss when adding the new models, the algorithm is called a gradient boosting algorithm. The strong learner is trained iteratively, starting with a base learner [39]. It is called gradient boosting because it uses a gradient descent algorithm to minimize the loss when adding new models. Both gradient boosting and XGBoost follows the same principle while the key differences between them 
lie in implementation details. XGBoost achieves better performance by controlling the complexity of the trees using different regularization techniques [34].

Let $\left(x_{1}, y_{1}\right),\left(x_{2}, y_{2}\right), \ldots,\left(x_{n}, y_{n}\right)$ be a set of input features and corresponding outputs. The tree ensemble algorithm uses $K$ additive functions, each representing a decision tree [36], to predict the output. The predicted output is given by the sum of each function prediction in Equation 2.7 [34], [39].

$$
\hat{y}_{i}=\sum_{k}^{K} f_{k}\left(x_{i}\right), f_{k} \in F,
$$

where $f_{k} \in F$ is the space of the decision trees. The given function is approximated by minimizing the regularized objective function for a given set of parameters $\theta$ given in Equation 2.8.

$$
\operatorname{obj}(\theta)=\sum_{i}^{n} l\left(\hat{y}_{i}, y_{i}\right)+\sum_{k}^{K} \Omega\left(f_{k}\right)
$$

where the first term $l\left(y_{i}, y_{i}\right)$ is the training loss function that measures the difference between the predicted output and the actual output. The training loss function can be measured using the Mean Squared Error (MSE), given by Equation 2.9 [23].

$$
M S E=\sum_{i}^{n}\left(y_{i}-\hat{y}_{i}\right)^{2}
$$


The second term $\Omega\left(f_{k}\right)$ is the regularization term, which penalizes the complexity of the model to avoid overfitting [41]. In XGBoost the regularization term is given in the equation 2.10 .

$$
\Omega(f)=\gamma T+\frac{1}{2} \lambda|w|^{2},
$$

where $T$ is the number of leaves, and the second term is the $L 2$ norm of leaf scores. During training, the model is trained additively, by optimizing for one tree at a time. Let $y_{i}^{t}$ be the prediction value at iteration $t$, and the additive procedure is given in Equation 2.11.

$$
\hat{y_{i}^{t}}=\sum_{k=1}^{t} f_{k}\left(x_{i}\right)=\hat{y_{i}^{t-1}}+f_{t}\left(x_{i}\right),
$$

where $y_{i}=0$. The tree added at each step is the tree that optimizes the objective function. The objective function can be rewritten in Equations 2.12 and 2.13 [41]:

$$
\begin{gathered}
o b j^{t}=\sum_{i} l\left(\hat{y}_{i}, y_{i}\right)+\sum_{k}^{K} \Omega\left(f_{k}\right) \\
=\sum_{i=1}^{n} l\left(y_{i}, y_{i}^{t-1}+f_{t}\left(x_{i}\right)\right)+\Omega\left(f_{t}\right)
\end{gathered}
$$

The objective function, given in Equation 2.12, is used as a scorer to evaluate the tree structure [41]. The XGBoost algorithm, with the help of the Sci-kit learn library and a summary of the tuned parameters is given below [32]: 
- Learning rate: represents shrinkage at every step (round) during boosting. The shrinkage parameter controls the penalization of each newly added tree to the model. However, using a low learning rate requires more rounds to find the optimal model.

- Num rounds: represents the number of rounds the model is boosted. In other words, how many estimators to use.

- Max depth: represents the maximum depth of a tree. Increasing the value of the tree depth makes the model more complicated.

- Min child weight: represents the minimum sum of instance weight in a child.

- Gamma: controls regularization. The minimum loss reduction required to make further partition on a leaf node of the tree and used to control pruning.

- Subsample and Column Subsample: controls the subsample ratios of the training instance. The ratio decides how much of the data XGBoost can select randomly.

Therein, XGBoost is a type gradient boosting implementation with high speed and predictive quality. The XGBoost is considered as faster as it natively supports parallel computing since a large part of its code back-end is written in $\mathrm{C}++$ [42]. The XGBoost is also called a regularized form of GBM (Gradient Boosting Machine), as L1 (Lasso Regression) and L2 (Ridge Regression) regularization prevents the model from overfitting. The main drawback of the XGBoost algorithm is the number of hyperparameters to be tuned for optimizing the model. 


\subsubsection{Convolutional Neural Network (CNN)}

Convolutional neural network $(\mathrm{CNN})$ is a variant of vanilla Multi-Layer Perceptions modelled after the visual cortex of animals [43]. CNN consists of stacks of convolutional layers and pooling layers which extract low-level features and have shown to learn higherlevel features progressively in the form of higher-level features in the form of filters in convolutional layers. CNN is particularly well suited for handling grid-like data where the data's structure contains information, such as audio signals, which are 1-dimension, images which are 2-dimension signals, and videos, which are 3-dimension signals. In many machine learning models, such as vanilla multi-layer perceptron, when handling data with structure, the vectorization of higher dimensional data into a single dimension is necessary. Reducing the data to a lower dimension compromises the information represented by the data. CNN is used to overcome this drawback, as mentioned above [44]. A CNN is made up of a combination of two layers, namely the convolutional layer and the pooling layer. The convolutional layers are essentially the learnable filters (weights of the networks) with an activation function. The pooling layers perform the process of subsampling. The key operation of convolution is performed by obtaining the dot product between subsection of the input data $I$ of size $n$ and the filters $F$ as given by Equation 2.16.

$$
F=\left[\begin{array}{ccc}
w_{11} & \ldots & w_{1 n} \\
\cdot & \ldots & \cdot \\
\cdot & \ldots & \cdot \\
w_{n 1} & \ldots & w_{n n}
\end{array}\right]
$$




$$
\begin{gathered}
I=\left[\begin{array}{ccc}
i_{11} & \ldots & i_{1 n} \\
\cdot & \ldots & \cdot \\
\cdot & \ldots & \cdot \\
i_{n 1} & \ldots & i_{n n}
\end{array}\right] \\
F \cdot I=\sum_{j=1}^{n} \sum_{k=1}^{n} w_{j k} i_{j k}
\end{gathered}
$$

The filter is made to slide over the image, convolving (dot product) the filter with each patch of the image as it slides. The number of sample points the filter moves each time its slides, is called as the stride length. CNN configurations can comprise a multitude of hidden layers. The four principal layer types that exist are

- Convolutional layer (CONV) + Activation (RELU): Convolutional filters are used to derive an activation map from the input data. The activation function, such as the Rectified linear unit (RELU), is used to provide only positive values for much faster training time.

- Pooling layer (POOL): Performs a non-linear down-sampling and reduces the number of weight parameters that are to be reduced.

- Fully connected layer (FC): Computes the class probability scores by outputting a vector of $\mathrm{N}$ dimensions, with $\mathrm{N}$ being the number of classes. As the name insists, all the neurons in this layer are connected.

The weight of the filters is constantly updated with the training process with the help of an optimizer using the technique of backpropagation [45]. The main drawback of the CNN model is that it is difficult to explain the features that are automatically extracted by the CNN filters. Further, CNN requires comparatively more examples to learn from, as opposed to other machine learning algorithms that are considered in this thesis. 


\subsection{Artery structure and mechanical property}

\subsubsection{Composition of the arterial wall}

Artery is composed of a variety of materials that give rise to their non-linear mechanical properties. Figure 2.6 shows a typical conduit artery composed of three distinct layers: tunica intima, tunica media, and tunica adventitia [18].

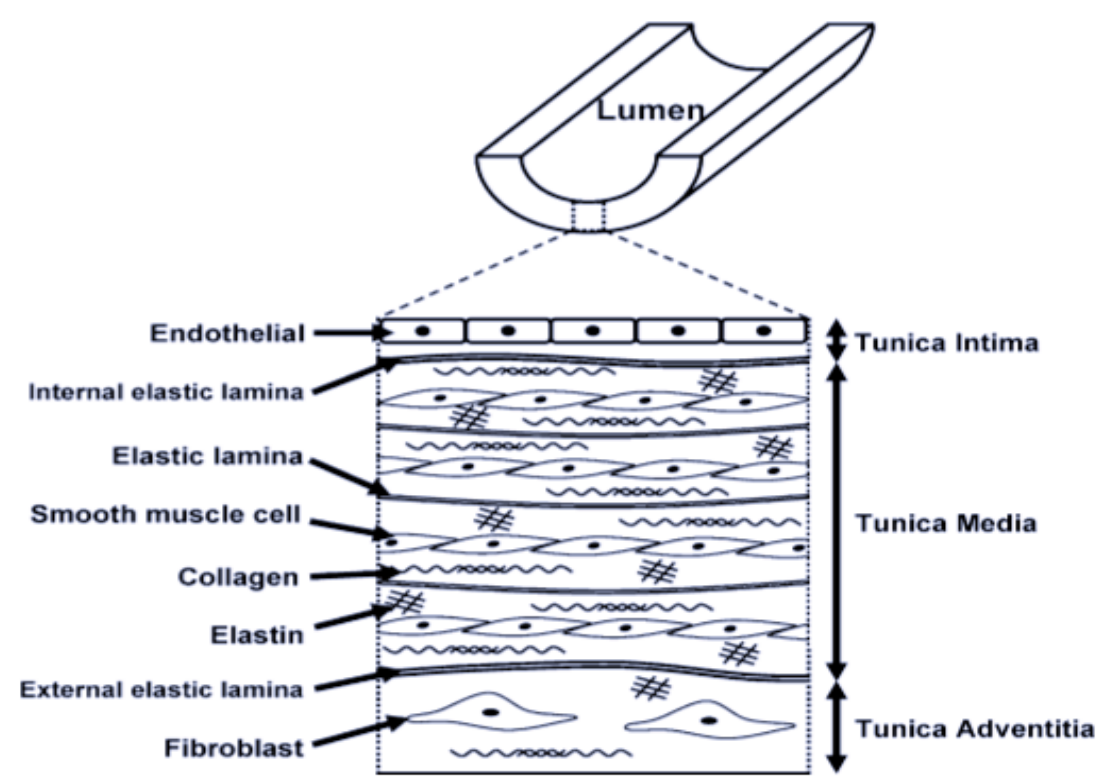

Figure 2.6 Cross-sectional view of a conduit artery wall. Adapted from [46].

The innermost layer from the inside of the artery (lumen) is known as the tunica intima and is comprised of elastic lamellae and endothelial cells. The primary function of the intima 
is to provide a smooth surface that promotes the flow of blood. The next outermost layer is the tunica media and is comprised of a combination of smooth muscle cells, elastic lamellae, and collagen fibers. Finally, the outermost layer, the tunica adventitia, is made up of mainly collagen and elastic fibers. Mechanical properties, such as Young's modulus of the arterial wall, are mostly determined by the tunica media and tunica adventitia [47][46]. At lower pressures, the distention of the arterial wall is governed primarily by the wall's elastin constituents, and it is because, at low pressures, the collagen fibers are initially unstretched [46]. As the pressure increases, the collagen fibers begin to engage and contribute a much higher tensile strength than the elastin components of the artery [47]. This combined action of the elastin and collagen components are the cause of the exponential increase in Young's modulus for an increase in arterial pressure or strain [47], [48].

\subsubsection{Common Carotid Artery}

The common carotid arteries are present on both the left and right sides of the neck and the head region. Figure 2.7 shows the schematic representation of the right common carotid artery along with their bifurcation into the internal and external carotid artery [24]. The left common carotid artery has two parts: a thoracic (chest) part and a cervical (neck) part. The right common carotid originates in or close to the neck and contains only a small thoracic portion [49]. The diameter of the common carotid in humans are ranging between 4.3 to $7.7 \mathrm{~mm}$ for both males and females. Further, the carotid artery diameter is significantly greater in males as compared to the female [50]. It is also observed that the height and 
weight of the person correlates with the diameter and thickness of the common carotid artery [51].

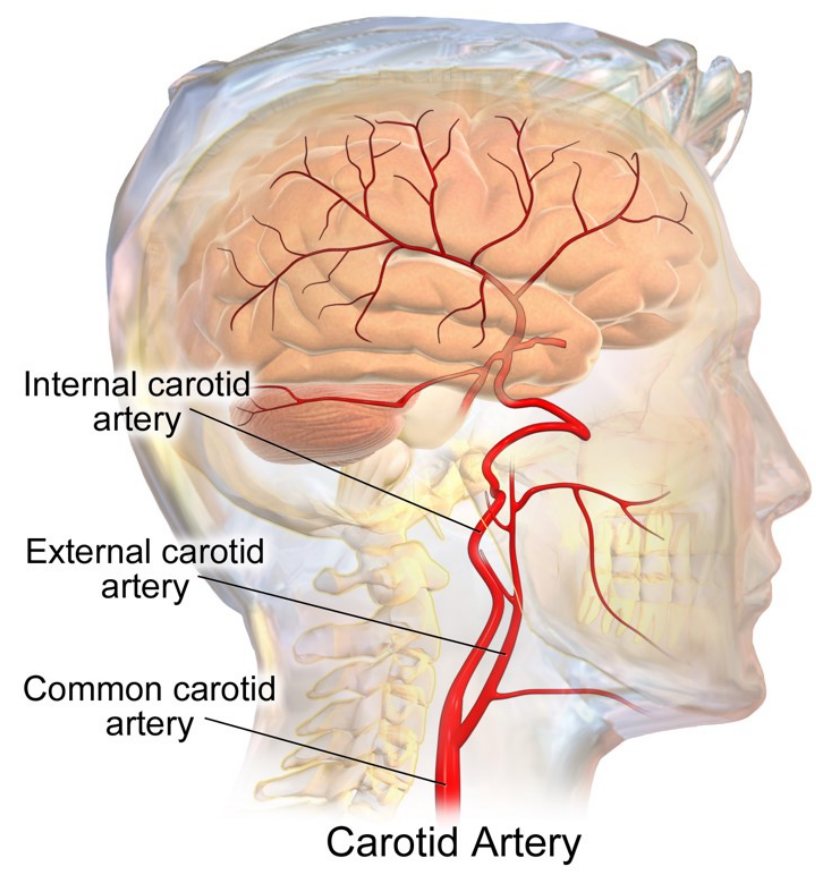

Figure 2.7 Schematic of the right common carotid artery, obtained from [52].

Depending on the individual, the common carotid artery is usually found in the region of depth ranging from 1.5 to $3 \mathrm{~cm}$ [51]. The test of vascular screening is carried on the common carotid artery to measure blockages in the arteries to the brain. This test is recommended for people with risk factors for vascular disease, such as high blood pressure and high cholesterol in the blood. It is necessary to regularly have the vascular screening tests as the arteries that are clogged become stiffer and can result in a brain stroke if left untreated. As the development of the clog in the arteries is a slow process, the early stage of the disease can be cured simply by correcting the lifestyle and eating habits [50], [53]. 
Thus constant monitoring of the change in the stiffness could be very useful in the prevention and diagnosis of vascular-related diseases, which leads to stroke in many cases.

\subsubsection{Arterial Stiffness and distension}

An overview of the mechanical properties of the arterial walls and the description of the metrics that relate to the arterial wall stiffness is explained in this section. It is important to understand hemodynamic principles, to appreciate the challenges associated with the estimation of the stiffness from the arterial diameter measurement by continuous monitoring using a non-invasive device.

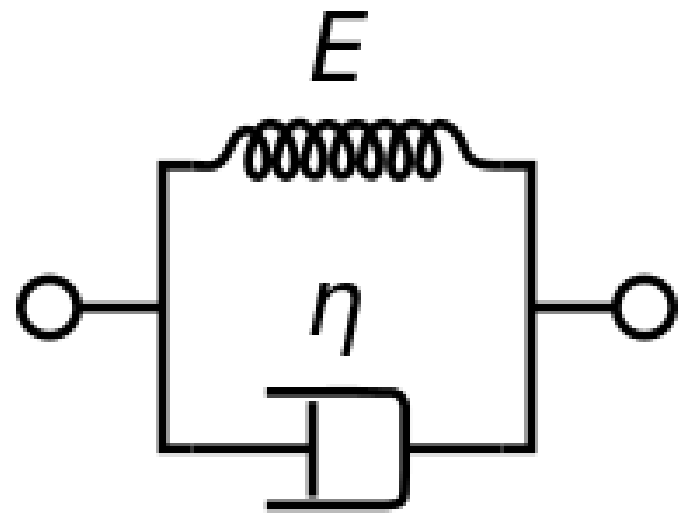

Figure 2.8 Schematic representation of the Kelvin-Voight model of

biological tissue. Adapted from [54].

The arterial wall is not purely elastic by nature but viscoelastic [47]. A viscoelastic substance is one that exhibits both elastic and viscous properties. The viscoelastic property of a material may be explained with the Kelvin-Voigt model, for instance, as shown in Figure 2.8 [26]. The Kelvin-Voigt model consists of a Newtonian damper and Hookean 
elastic spring connected in parallel. The major difference between the viscoelastic material and elastic material is that the dependency of its properties (such as deformation under applied stress) with time. A viscoelastic material such as the artery varies its stiffness depending on how rapidly the stress is applied. Due to this property, the arterial walls become stiffer, when a certain amount of pressure is applied in a very short period of time as opposed to the same amount of pressure applied over a longer period of time. Owing to this property of the arterial walls, the continuous monitoring of the change in the diameter helps to measure the stiffness of the arteries. Hooke's law is used to describe the proportionality between evenly distributed stress and strain for elastic materials, where stress is in units of force per area (pressure), and a strain refers to the change in material dimensions as a percentage of its original size. Since the arteries also show linear elastic properties, for certain applied stress, there is a proportional deformation or strain observed. The stress applied to the arterial walls due to the pressure exerted by the blood flow in the arteries; this constitutes the change in the lumen diameter of the artery. A constant of proportionality called elastic modulus or Young's modulus $(E)$ is used to determine the relationship between stress to strain. Young's modulus is defined as the slope of the stress/strain curve and has units of pressure (as strain is a dimensionless ratio). The determinant on the stress/strain curve gives the instantaneous modulus of elasticity for a small change in stress and strain. This quantity is known as the incremental Young's modulus $\left(E_{\text {inc }}\right)$ and is more useful for materials that have nonlinear stress/strain characteristics. Figure 2.9 shows an example of a nonlinear stress/strain curve, which is typical for large arteries [55]. 


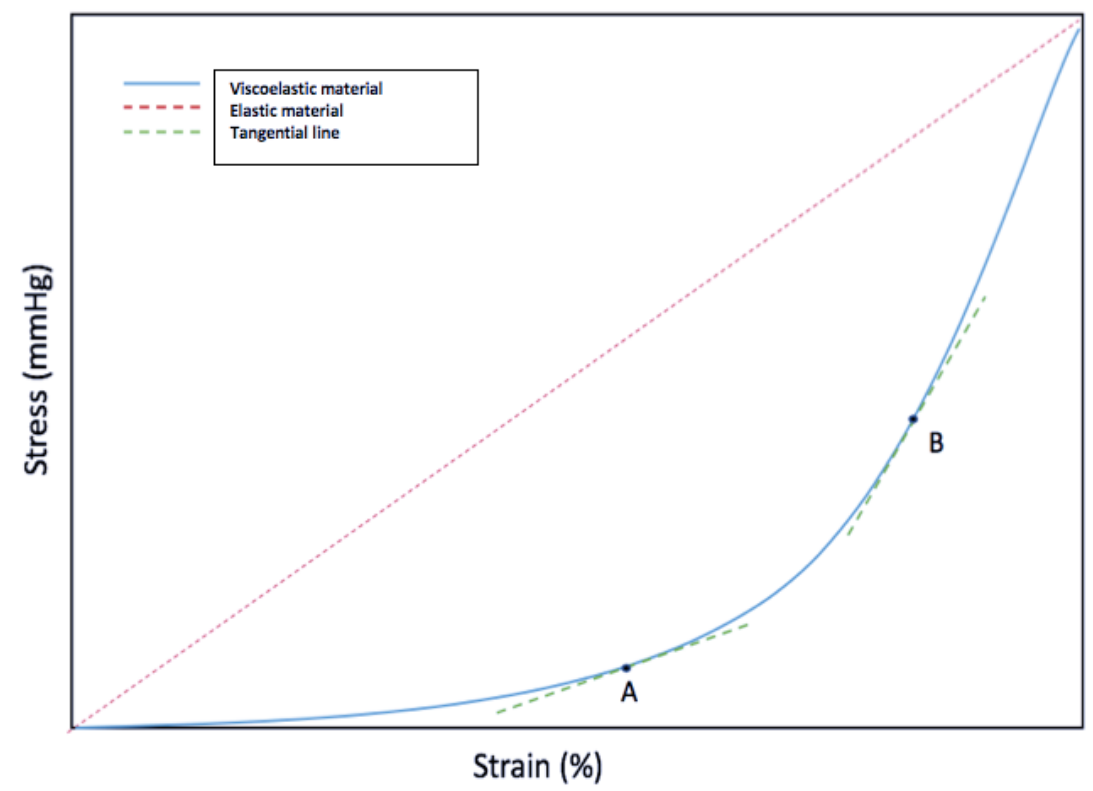

Figure 2.9 An example of stress versus strain curve of materials.

The Young's modulus of a perfectly elastic material is depicted as a straight red dotted line. However, the common carotid artery is a viscoelastic material, whose stress-strain relationship is given by the stress/strain curve (solid blue line) [28]. Furthermore, the nonlinear behavior of the common carotid artery is explained using the Kelvin-Voigt model. The incremental Young's modulus (green dotted line) is shown at two locations (A and B) being the slope at specific points on the stress/strain curve (blue). In this example, the arterial wall becomes stiffer (has a greater $E_{\text {inc }}$ ) as stress (pressure) or strain (distention) increases. 


\subsection{Artery diagnosis methods}

\subsubsection{Endothelial dysfunction and FMD measurement}

Endothelial dysfunction is defined as the defect in the production or activity of nitric oxide in the inner layer (endothelium) of the artery. The major indicator of endothelial dysfunction is impaired endothelium-dependent vasodilation [56]. The endothelial dysfunction is considered as an early marker for atherosclerosis. Atherosclerosis is a disease in which the plaque builds up in the inner wall of the artery. Over time, the plaque formed might harden up and narrow down the artery constricting the flow of blood, which could lead to fatal medical complications such as stroke and heart attack [57]. Thus, to assess the healthiness of the endothelial cells of the artery, the technique of flow-mediated dilatation (FMD) is used at the brachial artery with a cuff. The preferred choice of imaging modality to measure the FMD is ultrasound owing to its ionization radiation-free nature. The procedure for the experiment to measure the FMD of an artery is explained in detail in [58]. The calculation of FMD as a percentage change utilizes the peak diameter in response to reactive hyperemia with the baseline diameter, and is calculated utilizing the following Equation 2.17:

$$
F M D(\%)=\frac{(\text { Peak daimeter }- \text { Baseline diameter }) \times 100}{\text { Baseline diameter }}
$$

The baseline diameter is measured as the average of the systolic and diastolic diameter for at least ten cardiac cycles. Thus, the accurate measurement of the artery diameter is required to measure the FMD. 


\subsubsection{Artery stiffness}

The arterial wall stiffness of the common carotid artery is a very well-established indicator of cardiovascular disease [5], [7], [9], [50], [53]. Arterial healthiness can be evaluated from the parameters given in Table 2.1 [36]. Arterial stiffness index is a quantitative physiologic value to characterize the arterial stiffness. The higher the value of the stiffness index values, the higher the risk of coronary heart diseases [59], [60]. Arterial distensibility is a measure of the arterial ability to expand and contract with cardiac pulsation and relaxation [61]. Artery compliance is defined as the ratio of artery diameter difference and the pressure difference between the systolic and diastolic states [62]. The presence of the plaque in the arterial wall of the carotid artery can be identified by measuring the change in the stiffness of the artery [8]. 
Table 2.1 Parameters commonly used to diagnose artery

healthiness.

\begin{tabular}{|c|c|}
\hline Parameter & Definition \\
\hline Artery stiffness index & $\frac{\ln \left(\frac{P_{s}}{P_{d}}\right)}{\frac{\Delta D}{d_{d}}}$ \\
\hline Artery distensibility & $\frac{\Delta D}{\left(\Delta P \times d_{d}\right)}$ \\
\hline Artery compliance & $\frac{\Delta D}{\Delta P}$ \\
\hline \multicolumn{2}{|c|}{$\begin{array}{l}d_{s}: \text { systolic lumen diameter, } d_{d}: \text { diastolic lu } \\
\Delta D=d_{s}-d_{d} \\
p_{s}: \text { systolic pressure, } p_{d}: \text { diastolic pressure. } \\
\Delta P=p_{s}-p_{d} .\end{array}$} \\
\hline
\end{tabular}

From Table 2.1, it is seen that all the parameters require the measurements of the lumen diameter and blood pressure. To measure the systolic and the diastolic pressure, wellestablished methods, such as the auscultatory method [63], automatic oscillometric method[6],[7], applanation tonometry[65], Peňáz or vascular unloading method [64], [66], can be used. Since it is difficult to measure the change in pressure of the carotid artery owing to its location, the brachial artery pressure can be used interchangeably with the carotid artery pressure [67]. The various non-invasive, ionization radiation-free and cost- 
effective methods of the measurement of the arterial diameter measurement will be described in the following section 2.4.

\subsection{Measurement methods of artery diameter}

\subsubsection{Ultrasound measurement methods}

The following sections describe non-invasive, ionization radiation-free, and cost-effective methods that are used to measure the diameter of the artery. As mentioned in the above section, the tracking of the arterial diameter change can be used to calculate the stiffness of the arterial wall and the measurement of the FMD [68].

\subsubsection{B-mode ultrasound imaging}

The conventionally preferred method of the measurement of the change in the arterial diameter to evaluate endothelial dysfunction and identify the risks of heart diseases is using B-mode ultrasound imaging. It is the preferred method of choice, owing to the non-invasive nature of ultrasound and the cost-effectiveness as compared to the other imaging modalities such as X-ray and functional magnetic resonance imaging (fMRI) [69]. To eliminate the necessity of a trained radiologist to assess the B-mode imaging of the arterial walls, several new techniques such as semi-automatic and automatic measurement of the arterial wall diameter were proposed [18], [19]. However, it still requires the cost of the B-mode ultrasound array probe and ultrasound imaging system along with the computationally expensive software to automatically segment and locate the arterial walls for the accurate diameter measurement. 


\subsubsection{M-mode ultrasound image using a single UT}

In an attempt to retain the convenience of the conventional ultrasound method, and to reduce the cost and resource associated with the ultrasound B-mode imaging, a new technique using a single A-mode UT was proposed. ARTerial Stiffness Evaluation for Noninvasive Screening (ARTSENS) method was proposed by [20]-[22], which uses a lowcost single UT along with the necessary algorithm to locate and track the arterial wall locations using the M-mode image. Furthermore, the method was applied for mass screening of the patients in rural places of the country, and the proof of concept was verified. The use of a single UT could be a promising low-cost solution for the massscreening of coronary artery disease. Even though the proposed method could greatly cut the cost associated with the ultrasound hardware, the requirement of highly trained human operators to locate the UT on a patient and interpret the results still existed. The major issue with the use of a single UT is its difficulty in identifying the arterial walls, as there is no B-mode image guidance available. The semi-automated guidance technique to help the operator and automatically find and track the arterial walls was proposed in [20]. The shortcoming of the proposed method is that it is still necessary to manually provide the region of interest to locate the artery and track the diameter changes. A new automated guidance technique algorithm proposed in [70] requires the initial noise-free transmitted ultrasound signal to correlate the reflected echoes. The drawbacks mentioned above indicates that there is a need to develop a fully automated artery wall location and diameter tracking method using the ultrasonic signals acquired by a single UT in M-mode. It is also known that the cardiovascular diseases associated with the arterial stiffness are often referred to as 'sneaky disease' [12] since the disease is developed in a person over some 
time and not overnight [12]. Thus, the continuous monitoring of the artery properties could provide more useful information over a scheduled or random screening test. Owing to the rigidity of the lead zirconate titanate (PZT) ceramic single UT employed in ARTSENS, the motion artifact, steady UT contact on the skin surface of a subject, uneven pressure applied by the UT during measurement may deteriorate the estimation accuracy of the artery diameter. Also, long-term continuous monitoring may be difficult. To overcome the challenges mentioned above, a wearable ultrasonic sensor (WUS) could be developed and used for cardiovascular disease diagnosis at early stages [26], [71]. The WUS could be more effectively used if a reliable algorithm to detect and track the artery diameter is developed.

\subsubsection{Other low-cost methods}

The digital thermal monitoring (DTM) has been developed as an alternative to the ultrasound imaging method for the FMD, where the peripheral of the endothelial cells are evaluated by the change in the diameter of the brachial artery. This method has been proven to work by evaluating the measurement of vascular reactivity during a 5-minute arm-cuff reactive hyperemia test. The DTM also has the same advantage of non-invasiveness as that of the ultrasound, and very efficient.[23]. Furthermore, the relationship between the DTM and the cardiovascular risk factors was verified [72]. Since the DTM device primarily focuses on the FMD measurement at the brachial artery with the pressure cuff, the DTM cannot be applied for the measurement or the estimation of the change in diameter of the common carotid artery where the cuff pressure cannot be applied. 


\subsection{Summary}

As explained in this chapter, the stiffness of the artery associated with cardiovascular healthiness can be estimated by measuring the changes in the blood pressure and the diameter of the artery. Several methods to measure the blood pressure of the artery is discussed in Section 2.2.5. Further, a review of the existing non-ionization and costeffective methods to measure the diameter of the artery is presented along with their limitations. Among the different techniques to track the artery diameter, the ultrasonic method using a single UT is found to be efficient and cost-effective, and thus it is chosen in this thesis research. In this ultrasonic method, only a single UT without B-mode image guidance is used to locate and track the artery.

The main aim of this thesis research is to develop an automatic method to locate and track the arterial walls using the M-mode ultrasound signals, obtained by the single UT. In the next chapters, the design and construction of the experimental setup to compare different algorithms proposed for automatically locating and tracking the arterial walls will be outlined. Different approaches and algorithms to design a classifier to locate the arterial wall region in the M-mode image will be discussed, along with a technique to estimate the change in the diameter. The performance of the proposed algorithms will be investigated to find the best performing algorithm to detect and track the arterial wall diameter using ultrasound data obtained in phantom and in-vivo experiments. Finally, the algorithm developed will be tested on the M-mode signal obtained at the carotid artery of a human subject using the flexible WUS proposed for monitoring artery diameter [26], [71]. 


\section{Chapter 3: Ultrasound data acquisition System, phantom design and construction, and experimental setups}

This chapter is broadly divided into two sections: Section 3.1 describes the ultrasound data acquisition system, and Section 3.2 describes experimental environments, including phantom design and construction and experimental setups with the phantom and a human subject. Section 3.1 explains two different acquisition systems used in this thesis research: PICUS clinical ultrasound imaging system with a linear array ultrasonic transducer (UT) probe and the wearable ultrasonic sensor (WUS) system. It also gives an insight into the ultrasound signal specifications, system configuration, and the acquired ultrasonic data format for each system. Section 3.2 explains the two experimental environments used in this study: phantom experiments and in-vivo experiments. For the phantom experiments, the rationale of the phantom design, the procedure for the phantom construction, and the set up for the data acquisition with the PICUS clinical ultrasound imaging system are discussed. In the in-vivo experiments, the two different experimental setups using the PICUS clinical ultrasound imaging system and the WUS system are presented. Further, the differences between the ultrasonic signal data acquired by the two-acquisition system are analyzed and explained. 


\subsection{Ultrasound data acquisition system}

In this thesis, two different ultrasound acquisition system are used: (i) a clinical ultrasound imaging system with a linear array UT probe capable of acquiring B-mode image and raw ultrasonic rf signals over a period of time (M-mode signals) ; (ii) a wearable ultrasonic sensor (WUS) system capable of recording raw ultrasound rf signals over time (M-mode signals). Since the clinical ultrasound system provides a B-mode image and better SNR signal than the WUS system, the ultrasound data acquired from the clinical ultrasound system is used to develop and test the different algorithms explained in Section 4.1. After assessing the performance of the group of algorithms used in this thesis, the algorithm that performs the best is identified. The best performing algorithm is then evaluated by testing on the human in-vivo data acquired from both the clinical ultrasound system and the WUS system. The specifications of these ultrasound data acquisition systems are given in the following subsections. 


\subsubsection{Clinical ultrasound imaging system}

(A)

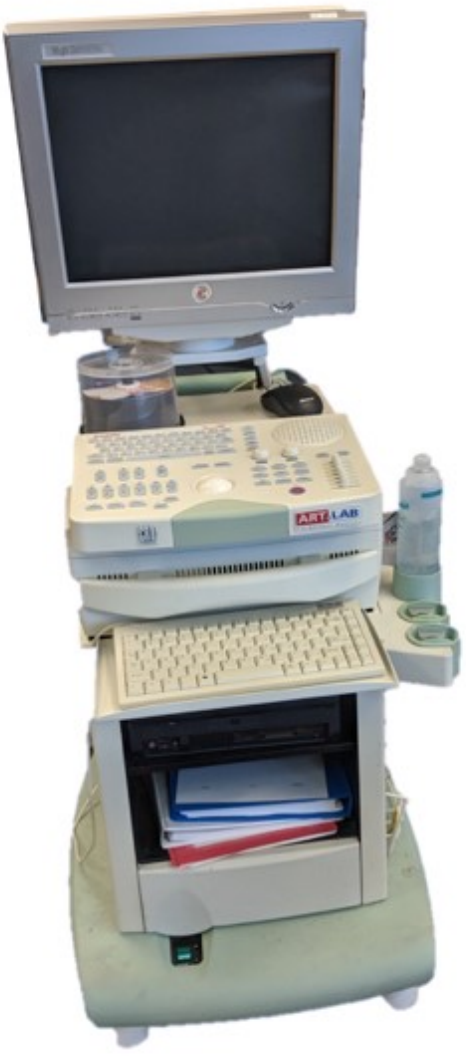

(B)

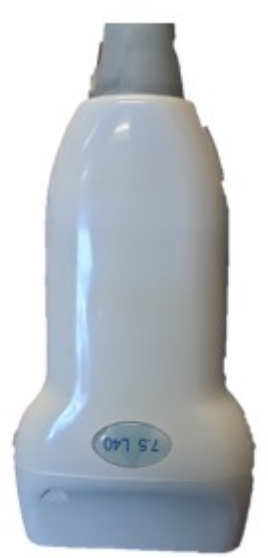

Figure 3.1 Photos of clinical ultrasound imaging system (A) and linear array UT probe (B) used in the experiments. 
The clinical ultrasound imaging system (Model: PICUS, ESAOTE Europe, Maastricht, Netherlands), shown in the Figure 3.1 (A), is used to acquire the ultrasound rf signal from the artery phantoms and the common carotid artery in this thesis. The data acquired from this system is used to develop and test the proposed algorithms for automatic artery detection and artery diameter tracking. The software used to view and acquire ultrasound rf signal data is called ART.LAB. PICUS ultrasound acquisition system can operate in Bmode and M-mode. Though the aim of the algorithm is to detect the arterial wall and track the wall diameter using ultrasound signal acquired in M-mode, theB-mode image is used to verify and confirm the position of the ultrasound probe over the artery. Furthermore, the B-mode image is also useds to create the ground truth about the location of the artery. As explained in Section 2.1.1.2, the B-mode image acquired is composed of a series of Amode signals, and the data from an individual A-mode scan line in B-mode data can be used for algorithm development, to be discussed in Section 4.1.

\subsubsection{Ultrasound probe}

The ultrasound probe of the PICUS ultrasound acquisition system employs a 40-mm wide linear array UT probe (Model: LI 0-5, ESAOTE Europe, Maastricht, Netherlands), as shown in Figure 3.1 (B). Figure 2.3 shows a cross-section of the probe. The probe consists of 128 UTs equally spaced. The M-mode image data is composed of 128 A-mode scan line data, and thus the spatial interval between two consecutive A-mode scan lines in B-mode image data is $315 \mu \mathrm{m}$. 


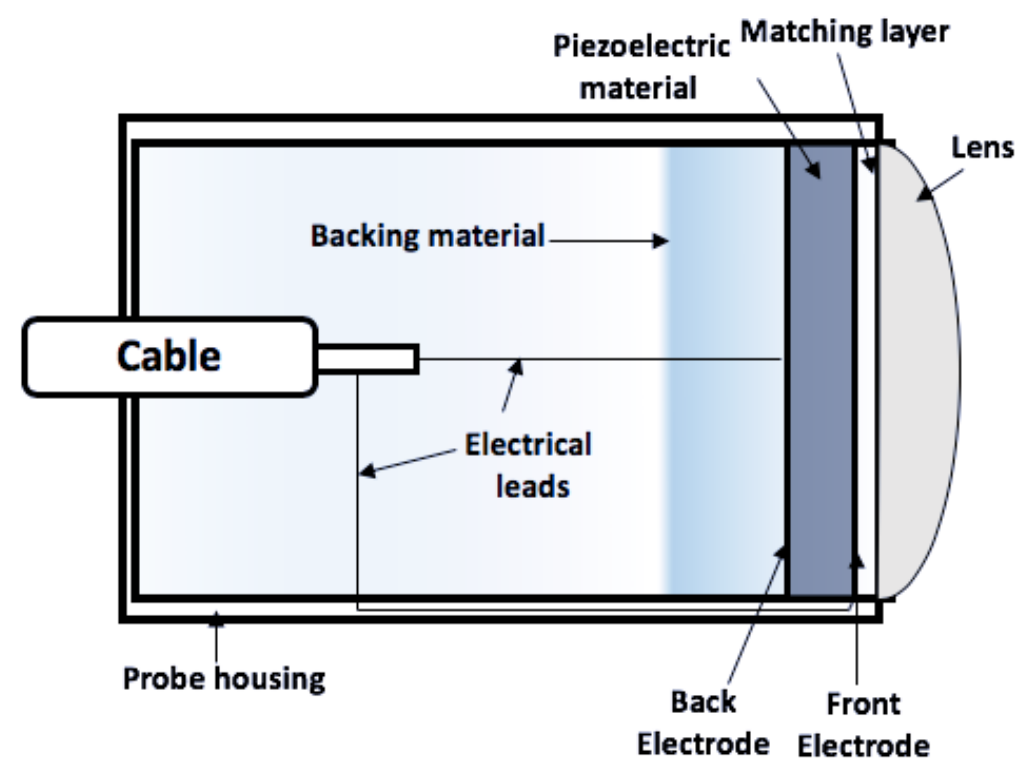

Figure 3.2 Cross-sectional view of the ultrasonic probe. Based on

The probe consists of the piezoelectric crystal that is responsible for the ultrasound excitation and reception. The piezoelectric material generally used for UTs in medical ultrasound systems is a ceramic material such as lead zirconate titanate (PZT). The piezoelectric material is sandwiched between two electrodes made of a good conductive material such as gold or silver. The electrodes are connected to an ultrasonic power delivery system, such as the ultrasonic pulse/ receiver. The impedance matching layer is aimed to optimize the ultrasound wave transmission between the probing surface and biological tissue. The final layer is an acoustic lens utilized to focus ultrasound waves into the medium under inspection. The backing material attached to the backside of the piezoelectric material absorbs the backward traveling acoustic waves from the piezoelectric material to reduce the internal acoustic noise. It also has a damping effect, which increases the 
frequency bandwidth of the ultrasonic pulsed signal to excite a pulsed ultrasound to accomplish a high spatial resolution along the depth direction. Electronic beam focusing and scanning technique is used to obtain the B-mode image with high spatial resolution along the lateral direction.

\subsubsection{Ultrasound Signal specification}

The PICUS system supports three major imaging modes: M-mode, B-mode, and Fast-B mode. The imaging mode used for the following experiments with the PICUS system is Bmode. The B-mode scan contains 128 A-mode scan lines in 40-mm lateral width, acquired with the linear array UT probe. The center frequency of the transmitted ultrasound pulsed signals can be selected in the range of 5-10 MHz. For the case of vascular imaging, the center frequency of 7.5 MHz is typically selected [56], [74]. B-mode signal is acquired at the B-mode frame rate of $30 \mathrm{~Hz}$. The frame rate is the number of B-mode frames acquired in a second. It is noted that along with the B-mode image that is displayed in the display of the PICUS clinical ultrasound imaging system, the corresponding ultrasonic rf signal data of A-mode scan lines are also stored.

\subsubsection{Data Acquisition software and data format}

The ultrasound system stores the digitized ultrasonic rf signal acquired by the ARTLAB software developed by Esaote in a ". zrf" file format. Esaote created this format and permitted for reading using a function called "readzrf .m," which was written in MATLAB. As the ultrasound $\mathrm{rf}$ signal analysis and processing in this thesis is carried out using the 
Python programming language, a new function called "readzrf .py" was written based on the original MATLAB file provided. The rf signals are stored in a circular buffer in the internal memory of the ARTLAB processing unit. In the circular buffer, the data is stored in a file with a ". zrf" extension. The "readzrf .py" is used to read the ". zrf" data files. The clinical probe is primarily used in the B-mode acquisition over time and the structure of the ultrasound rf signal acquired is given in Figure 3.3.

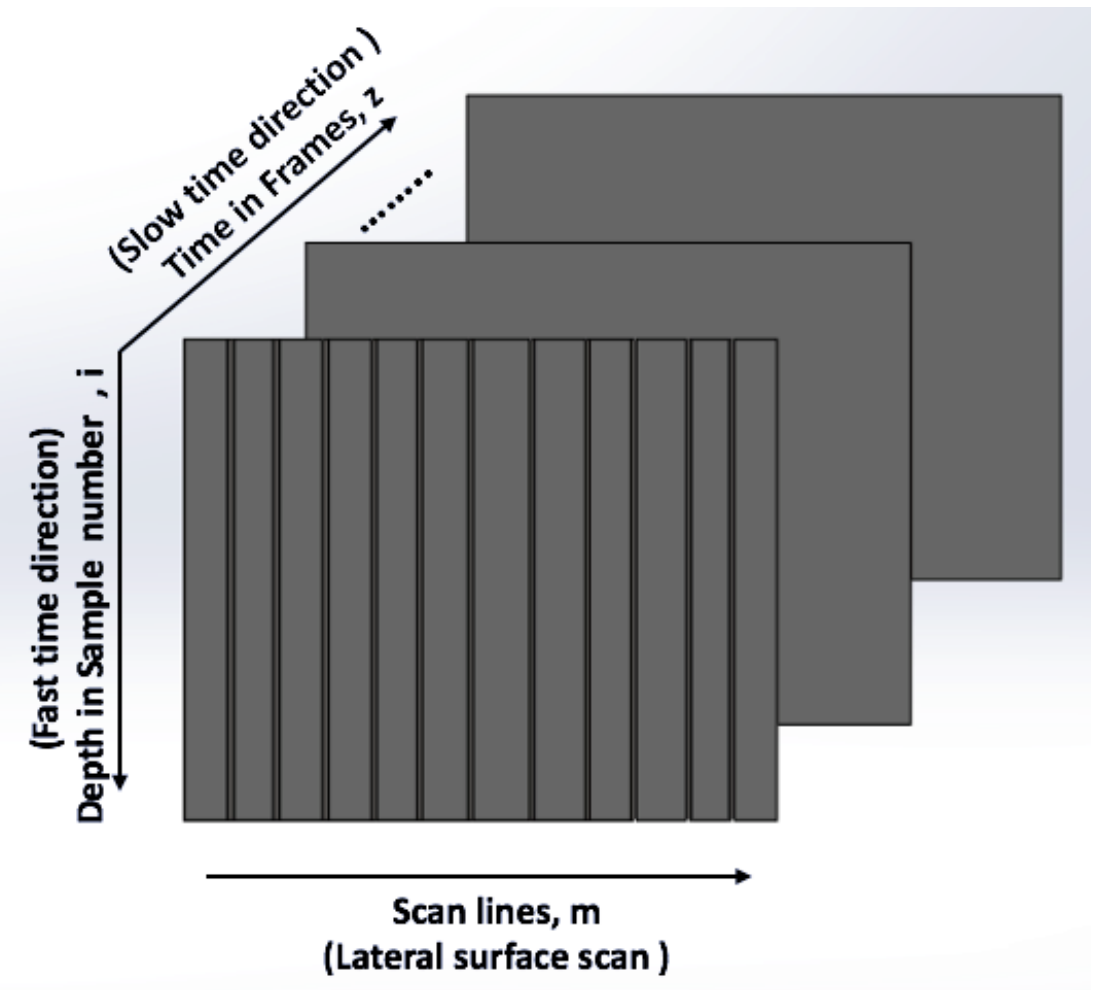

Figure 3.3 Data Structure of the ultrasound rf signals acquired in B-mode by the PICUS clinical ultrasound imaging system used in the experiments.

The lateral direction or the surface scan in B-mode is composed of 128 A-mode scan lines (i.e., index number $m$ ranges from 0 to 127). The fast-time direction refers to the depth of 
the medium through which the ultrasound is passing through. The value of sample number $i$ ranges from 0 to 1516 (see the M-mode rf ultrasound signal image in Figure 3.9). The sampling interval of the A-mode rf ultrasound signal is $30 \mathrm{~ns}$ (the sampling frequency is 33.3 MHz). Assuming that the average speed of the ultrasound in the soft human tissues is $1540 \mathrm{~m} / \mathrm{s}$, the total depth of the B-mode image (as well as M-mode) is $35 \mathrm{~mm}$.

It is noted that the B-mode image, displayed in the PICUS clinical ultrasound system, has half of the sampling rate of the rf ultrasound signal obtained. Thus, the range of the sample number used in the B-mode image to display the same depth of $35 \mathrm{~mm}$ ranges is from 0 to 758 (see the B-mode image in Figure 3.9). The slow-time direction represents the number of B-mode frames acquired over time. Since the frame rate of the B-mode acquisition is 30 $\mathrm{Hz}$ and the acquisition duration is 6 seconds in the PICUS system, the value of $\mathrm{z}$ representing the frame number ranges from 0 to 180. As explained in Section 3.1.1, each frame of B-mode data is composed of 128 A-mode scan line data. Therefore, in B-mode data, there are $128 \mathrm{M}$-mode signals acquired for 6 seconds, along the B-mode scan (lateral) direction.

\subsubsection{Wearable ultrasonic sensor (WUS) system}

Despite that the WUS system using a single UT can acquire ultrasound rf signal only in Mmode but not in B-mode, WUS could have several advantages for continuous, long-term and cost-effective monitoring of artery, over the PICUS clinical ultrasound imaging system with a linear array UT probe. The conventional ultrasonic probe used in medical imaging is bulky, rigid, and heavy. A rigid and wide B-Mode ultrasonic probe surface is not usually 
in perfect contact with the uneven and curved body surfaces, which requires the user to apply pressure to remove air gaps and to achieve a steady contact between the body surface and the probe surface. Moreover, the amount of pressure applied on the skin surface during the acquisition of the signal varies according to the examiner. This pressure applied could deform the original arterial shape and result in the diameter of the collapsed artery which is not very useful in most cases. Thus a flexible WUS proposed in [26], [28], is used in this thesis.

\subsubsection{System configuration}

The WUS system used in this thesis consists of a WUS, an ultrasonic pulser/receiver, a digitizer, and a computer. The configuration of the system components and its connections employed in the experiments for this thesis research are shown in Figure 3.4. 


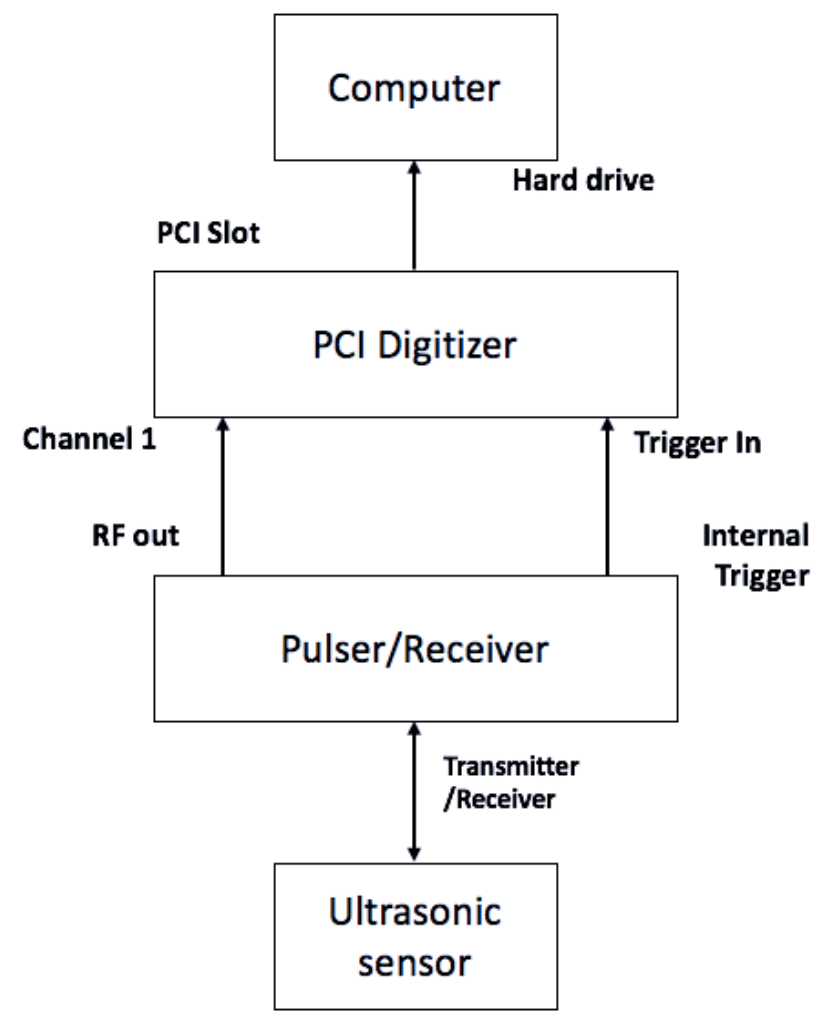

Figure 3.4 Block diagram of the WUS system used in the experiments. 


\subsubsection{Wearable ultrasonic sensor (WUS)}

(A)

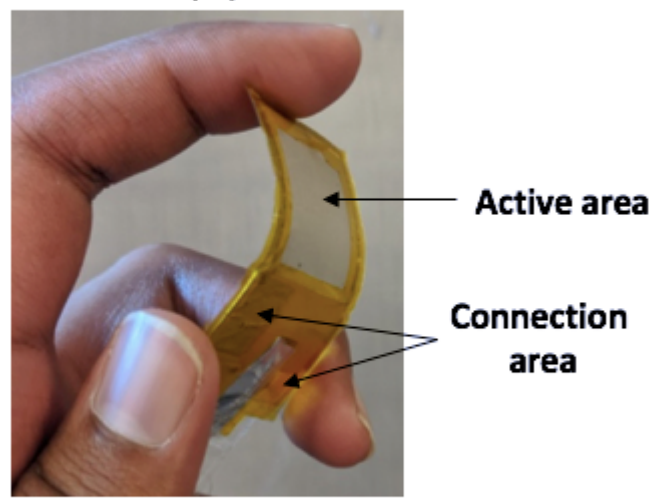

(B)

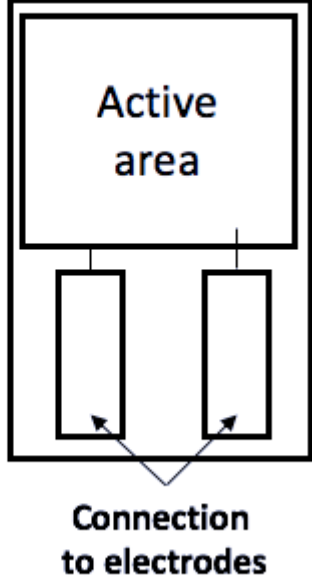

Figure 3.5 Photo (A) and schematic (B) of the wearable ultrasonic sensor (WUS) used in the experiments.

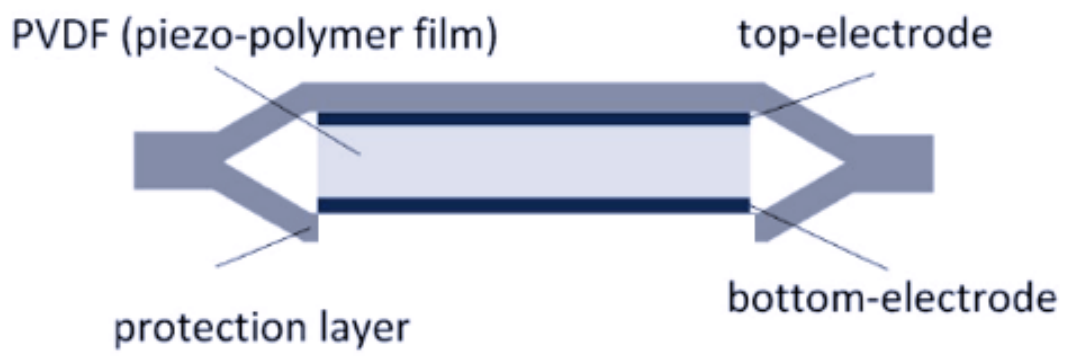

Figure 3.6 Schematic representation of a cross-section of the WUS. Adapted from [73]. 
Figures 3.5 and 3.6 show the WUS [73] developed in the Carleton biomedical ultrasound laboratory and used in this thesis research. The wearable ultrasonic sensor was made from a piezoelectric polymer [28] of polyvinylidene fluoride (PVDF), which is flexible and lightweight. The WUS was composed of three materials: the piezoelectric PVDF, the electrodes and the protection films. It has an active ultrasonic area of 20 by $20 \mathrm{~mm}$ which is the overlapped area of the top and bottom electrodes. The PVDF film was $110 \mu \mathrm{m}$ in thickness. The total thickness of the sensor was $450 \mu \mathrm{m}$, and its weight was $0.3 \mathrm{~g}$, including the electrical leads. The WUS is used in the experiments of artery wall detection and tracking in this research. The estimated center frequency of the WUS sensor used in this thesis is $4 \mathrm{MHz}$.

The ultrasonic pulser/receiver (Model: DPR 300, JSR Ultrasonics, Pittsford, NY, USA) produces electric pulses that excite the WUS to generate a pulsed ultrasonic wave and receives the electric signal back from the WUS. The data acquisition (DAQ) system consists of two parts: PCI digitizer (Model: ATS 460, Alazar Tech, Montreal, QC, Canada) to acquire the signals from the ultrasonic pulser/receiver and DAQ software. The digitizer has a 14-bit resolution and a sampling frequency ranging from $10 \mathrm{kHz}$ to $125 \mathrm{MHz}$. The M-mode frame rate is set to 1000 frames per second by a function generator. It stores the data into the storage of a PC. The program, written in Python, analyzes the acquired ultrasonic data. 


\subsection{Experimental environments}

The performance of the algorithms (to be discussed in Chapter 4) for automatic artery detection and lumen diameter tracking are accessed with phantom and in-vivo experimental environments. The following section described artery phantom and human in-vivo experimental environments. In the artery phantom experiments, the design, construction, and acquisition setup of two types of phantoms are explained. For the human in-vivo experiment, the experimental setup is discussed.

\subsubsection{Artery Phantoms}

To investigate the performance of the arterial wall region classifier and the lumen diameter tracking methods, ground truth about the location of the artery wall is necessary. Since the location, dimension and the distensibility of the arteries varies according to individuals, a phantom with the known parameters is developed to test and compare the performance of the classifiers. The following section explains the design and construction of two types of phantoms named "Phantom A" and "Phantom B". The main difference between phantoms $\mathrm{A}$ and $\mathrm{B}$ is that the Phantom $\mathrm{A}$ uses water to be the surrounding tissue material of the polymer tube simulating the human artery, while the Phantom B uses gelatine-based hydrogel as a surrounding tissue material. The acquisition setup for both the phantom condition is also explained in the following subsection.

\subsubsection{Phantom experimental environment}

Figure 3.7 shows a schematic design of the phantom experimental environment. Two types of phantom experimental setups were designed and constructed: one with water (Phantom 
A) and another with a hydrogel (phantom B), surrounding a polymer tube that mimics an artery. The hydrogel is often used for a soft tissue-mimicking phantom material. The distance of the artery mimicking polymer tube from the ultrasonic probing surface is in the range of 10-30 $\mathrm{mm}$ as most of the carotid and brachial arteries are found in that range. The polymer tube is made of polysiloxanes. It has an inner diameter of $9.48 \mathrm{~mm}$, and the tube wall thickness is $1.25 \mathrm{~mm}$. The polymer tube simulating the artery wall was selected such that it is compliant enough to expand and contract when water is pumped into it by a syringe, to simulate blood flow. The other end of the tube is closed. In order to simulate the artery diameter change due to the blood flow in the cardiac cycle, water is pumped into the tube to expand the tube diameter by pushing the piston of the syringe, and then the excessive water causing the expansion of the tube is pumped out from the tube by pulling the piston of the syringe. As the main aim of the experiment is to simulate the expansion and contraction of the human artery, the piston was gently and consistently pushed and pulled in one second (one beat per second: 1bps). With the help of a B-mode image from the PICUS clinical ultrasound system, care was taken to expand and contract the polymer tube by the same amount. 


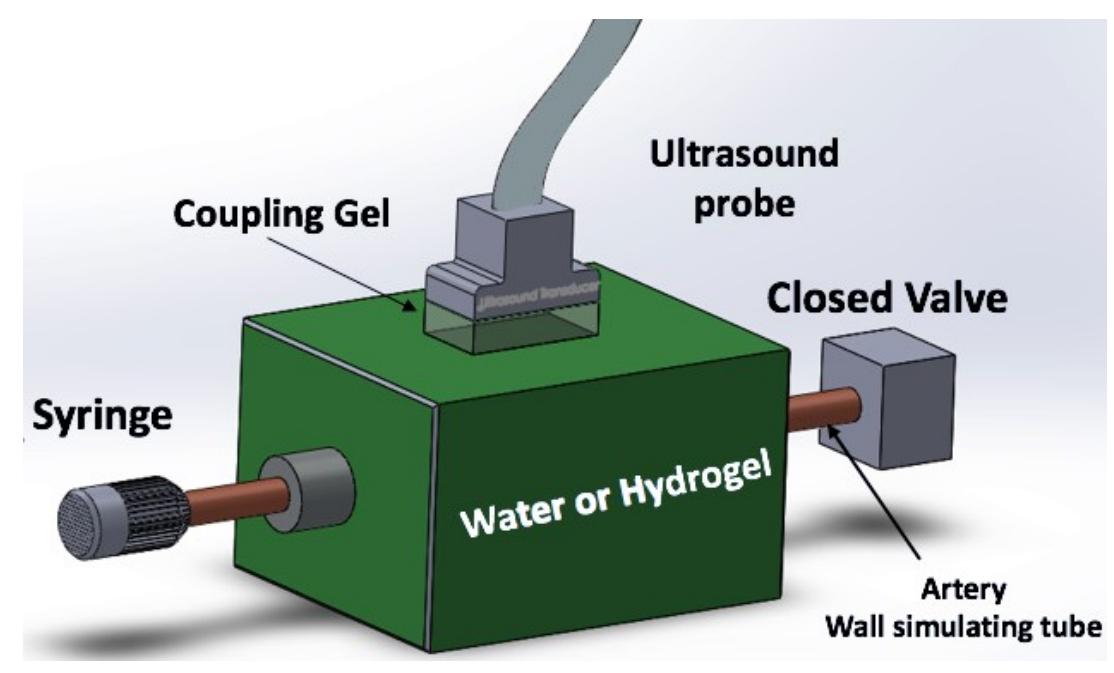

Figure 3.7 Schematic view of the phantom experimental

environment.

In the phantom experiments, the PICUS ultrasound imaging system with the ultrasound linear array probe was used to acquire B-mode data of ultrasound rf signal. Then, the Mmode data were retrieved from the acquired B-mode data to evaluate the performance of the proposed algorithms for artery detection and diameter tracking. Since the B-mode scan direction is along the longitudinal axis (length direction) of the polymer tube, one B-mode frame data contains $128 \mathrm{M}$-mode rf ultrasound signals along the length direction of the polymer tube. The B-mode frame rate is $30 \mathrm{~Hz}$.

\subsubsection{Phantom A}

The purpose of Phantom A is to produce an ideal experimental environment where there are no ultrasound scattering noises from the material (water) surrounding the artery (polymer tube) to test the proposed algorithms of artery wall region detection and the lumen diameter tracking. Hence, a polymer tube mimicking the artery is immersed in a water bath. 
Figure 3.8 shows the experimental setup of the water-bath based Phantom A for the ultrasound rf signal data acquisition using the PICUS clinical ultrasound imaging system. The upper side of the tube that is closer to the ultrasound probe is considered as the proximal wall, while the lower side is considered as the distal wall. Water is pumped manually by the syringe to mimic a human heartbeat at $1 \mathrm{bps}$.

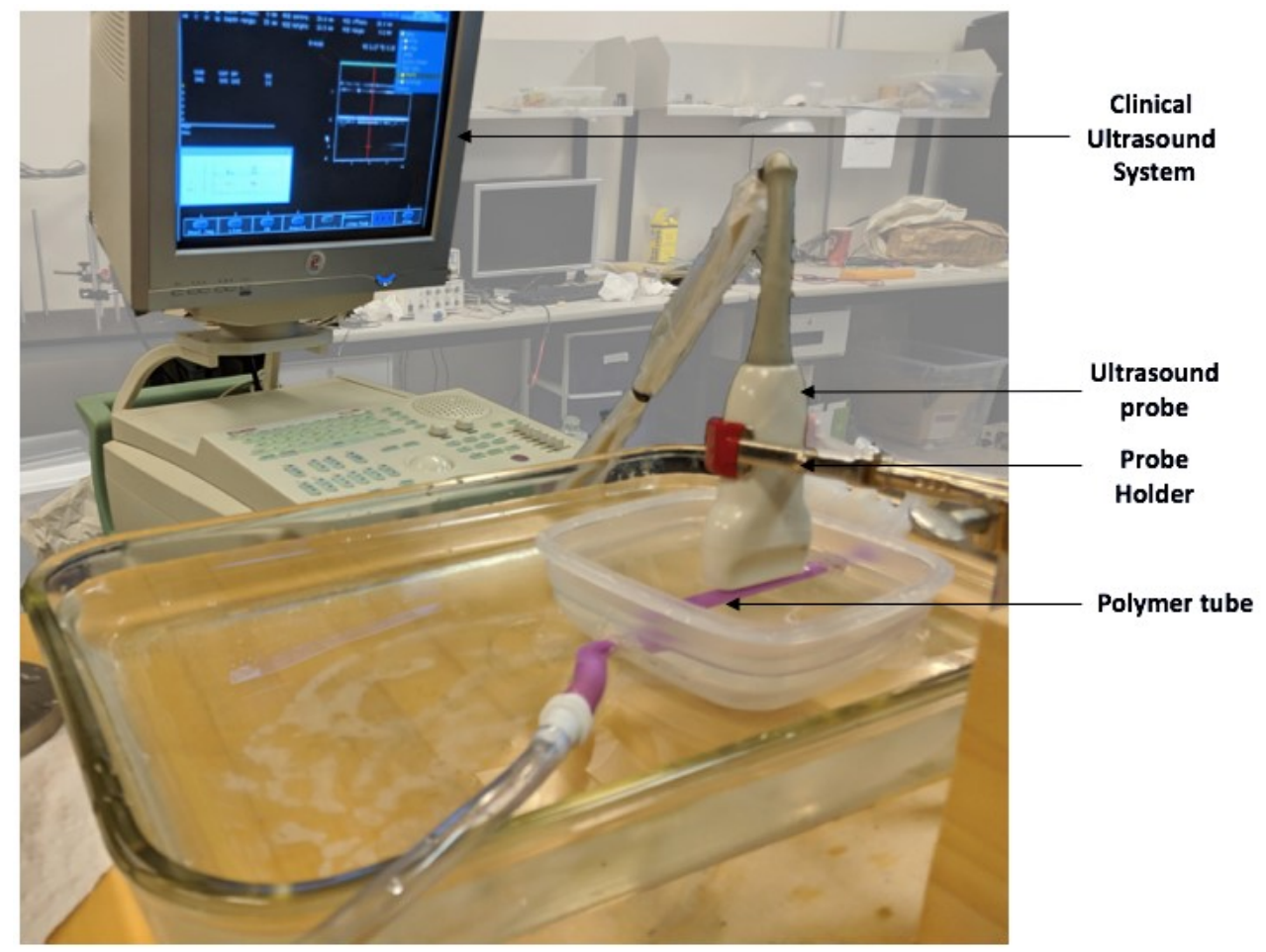

Figure 3.8 Experimental setup for data acquisition with Phantom A.

It is seen in Figure 3.8 that the Phantom A is immersed in a larger water bath to avoid air bubbles inside the polymer tube while pushing/pulling the piston of the syringe, which would create artifacts. The ultrasound probe is held steadily above the polymer tube using a probe holder. The ultrasound probe is located with the help of the B-mode image from the PICUS ultrasound imaging system such that the B-mode scan line is along the length 
and over the center of the polymer tube. The speed of the ultrasound in water at room temperature was obtained $1481 \mathrm{~m} / \mathrm{s}$. An example of a selected frame of the B-mode image is shown in Figure 3.9 (a). Figure 3.9 (b) shows the M-mode rf signal image retrieved from the acquired B-mode rf signal data corresponds to the scan line of \#60 in Figure 3.9 (a). It is noted that the maximum sample number in depth of the B-mode image is 758 in Figure 3.9 (a), while that of the M-mode rf signal image is 1516 in Figure 3.9 (b), due to a half of the sampling frequency for the amplitude (envelope) B-mode image data, comparing that of the ultrasound rf signal stored in the PICUS system, as explained in the Section 3.1.1.3. 


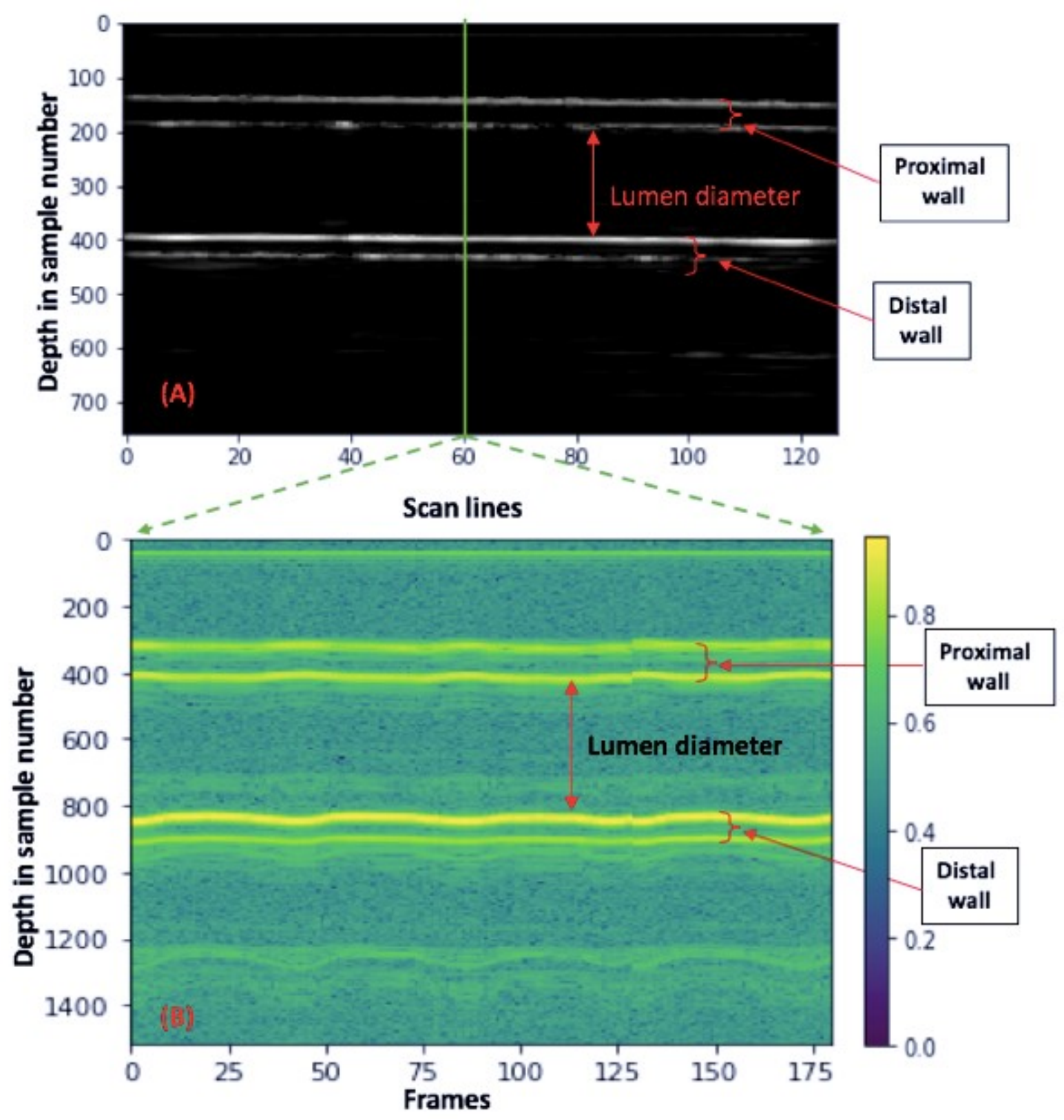

Figure 3.9 B-mode image of Phantom A (A) and the corresponding M-mode rf ultrasound signal image at the scan line of \#60 (B).

In order to differentiate the M-mode image, which shows the movement over time from that of the still B-mode image in the B-mode acquisition, the M-mode rf signal image of a 
selected B-mode scan line is displayed with the Viridis color palette henceforth in this thesis. It is observed in Figure 3.9 (B) that the M-mode image shows that the proximal walls at a depth of around 300 (sample number) and distal walls at 800 (sample number) move opposite to each other with the time (frames), indicating the expansion and contraction of the polymer tube diameter due to the water pressure changes inside the tube. It is noted in the M-mode image that along with the movement of the proximal and distal walls, there is also a similar pattern observed at a depth around at 1250 . This observed signal is due to the reverberation artifact. This artifact occurs when the ultrasound beam encounters two closely spaced reflective surfaces. A highly reflective surface or interface close to the transducer will cause most of the ultrasound to be reflected to the probe, due to the high amplitude of the echo a part of the sound will be reflected at the surface of the probe and back at the interface. At the interface, the ultrasound will be reflected back to the probe again, and at the UT, some of the ultrasound will be detected, and some will be reflected again. This back and forth reflection, i.e., reverberation, will continue until the energy of the ultrasound has been fully attenuated [75]. Since such an artifact signal is well separated from the desired signal from the artery walls, it does not affect to evaluate the proposed algorithms (as discussed in Chapter 4).

\subsubsection{Phantom B}

The purpose of Phantom B is to produce a more realistic experimental environment where there are ultrasound scattering noises from the tissues surrounding the artery. Hence, a polymer tube is surrounded by hydrogel-based phantom specimens with ultrasound scatterers are designed and constructed. Since there are always scattering noises (speckle 
echoes) observed in ultrasound signals and images acquired with a human subject, graphite powder, having a nominal particle diameter of $50 \mu \mathrm{m}$, is dispersed in the hydrogel phantom as ultrasound scatterers. Several phantom fabrication techniques, proposed in [76]-[81], were reviewed, and a combination of techniques suited for the experiments in this thesis is used. The materials and their composition to construct the hydrogel-based phantom are given in Table 3.1.

Table 3.1 Constituents of phantom B.

\begin{tabular}{|c|c|}
\hline Materials & Weight by percentage \\
\hline Gelatine & $35 \mathrm{~W} \%$ \\
\hline Distilled water & $43 \mathrm{~W} \%$ \\
\hline Isopropanol & $10 \mathrm{~W} \%$ \\
\hline Graphite powder & $2 \mathrm{~W} \%$ \\
\hline Silicone oil $(1000 \mathrm{cSt})$ & $10 \mathrm{~W} \%$ \\
\hline
\end{tabular}

As shown in Table 3.1, the major constituents that provide the viscoelastic nature to the phantom is a mix of gelatine and water. Isopropanol is added to the phantom to extend the longevity of the phantom by preventing the creation of moulds. The graphite powder acts as an ultrasound scatterer, which is the source of the speckle echoes. The silicone oil added helps to take out the phantom from the mold. It also increases the elasticity of the phantom. The amount of each constituent is based on the previous research given in [76]. 
The detailed construction procedure of Phantom B is given below.

1. The concentration of gelatine in the phantom (which would determine the stiffness of the phantom) is decided, and the ingredients are prepared accordingly.

2. Distilled water is boiled for 30 minutes for degassing.

3. The degassed water is heated and maintained at a temperature of $90^{\circ} \mathrm{C}$.

4. While the water is being stirred with a magnetic stirrer, gelatine is added slowly to the water to prevent them from forming lumps.

5. The solution is continuously mixed until the gelatine powders are completely dissolved (the solution becomes transparent). The time required for this process is subject to the amount of the solution.

6. Graphite powder is slowly mixed into the solution to prevent them from forming lumps. The graphite particles are added as the ultrasound scatterers.

7. The solution is stirred for another hour to ensure uniform distribution of the scattering particles.

8. Silicone oil of $1000 \mathrm{cSt}$ is added to the solution in order to aid the phantom from quickly taken out from the mold and to add more elasticity to the phantom.

9. Mixed a certain amount of alcohol into the mixture to prevent the growth of bacteria to extend the lifetime of the phantom.

10. The heating is stopped, but the solution is kept under constant stirring to avoid any settlements until the temperature drops below $60^{\circ} \mathrm{C}$.

11. The mold containing the phantom is transferred into the ice water bath to accelerate solidification. 
12. The mold is now transferred into the fridge from the ice water bath and waited for 24 hours to let it become solidified.

13. The solid phantom is removed from the mold and usually preserved in a plastic wrap to minimize the evaporation of water content. The phantom is then preserved in the refrigerator.

The phantom is taken out from the fridge a few hours before the experiment to ensure that it is at room temperature during the time of the experiment. This procedure was essential since the viscoelastic properties, and sound speed of the phantom varies with the temperature. Based on the observation of constructed multiple phantoms, the gelatine based hydrogel phantom lasted longer with the addition of the isopropanol and silicon oil as they prevented the formation of mould and reduced the evaporation of water from the phantom. A photo of the constructed Phantom B, mimicking the artery and surrounding soft tissue, is shown in Figure 3.10. The same polymer tube as the Phantom A is embedded in the constructed hydrogel phantom to mimic the artery. In Phantom B, the polymer tube is first inserted into the mould, and the hot tissue-mimicking gelatin-based phantom in liquid state is poured over/around the tube and allowed cool down. Once the phantom is solidified around the polymer tube, the mould is broken down to obtain the phantom, as shown in Figure 3.10. It is to be noted that the artery wall simulating polymer tube has been slightly deformed due to the phantom material surrounding the tube. The inner diameter of the polymer tube in this experiment is measured to be $9.55 \mathrm{~mm}$, as opposed to the original 9.48 $\mathrm{mm}$ of the polymer tube. 


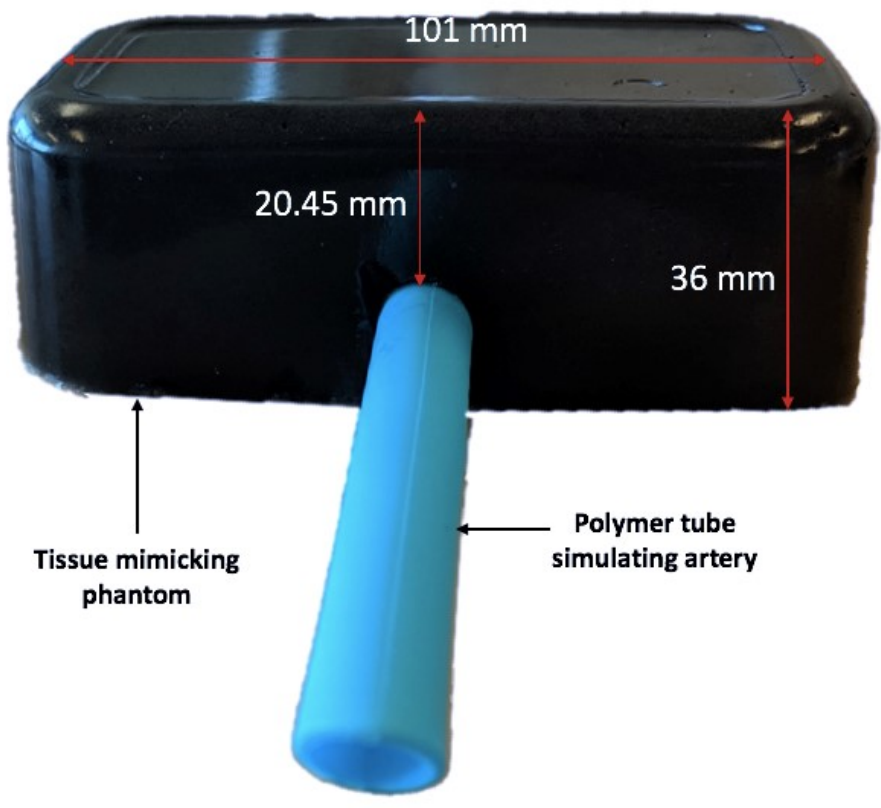

Figure 3.10 A photo of the constructed Phantom B, hydrogel-based tissue-mimicking artery phantom.

The speed of ultrasound in the constructed gelatine-based phantom was measured to be $1704 \mathrm{~m} / \mathrm{s}$. It is noted that precisely adjusting the acoustic properties such as the density, speed, and attenuation of the phantom was difficult and beyond the scope of this thesis. Therefore, the fabricated phantoms used in this thesis research were not adjusted to meet the recommended ultrasound properties for biological soft tissues (ultrasound velocity around $1540 \mathrm{~m} / \mathrm{s}$, and attenuation around $0.5 \mathrm{~dB} \cdot \mathrm{cm}^{-1} \cdot \mathrm{MHz}^{-1}$ ) [29]. The purpose of the phantom experiments was to test the algorithms of artery detection and diameter tracking, but not the fabrication of the ideal tissue-mimicking phantom. The constructed phantom is used in the experiments to verify the proposed algorithms of artery wall detection, tracking, and diameter estimation. The experimental setup with Phantom B is shown in Figure 3.11. 
Similar to the experimental setup with the Phantom A, the ultrasound probe was placed over the polymer tube simulating the artery with the help of the B-mode image using the PICUS clinical ultrasound imaging system. The water is pumped into the polymer tube with the help of a syringe as same as the Phantom A.

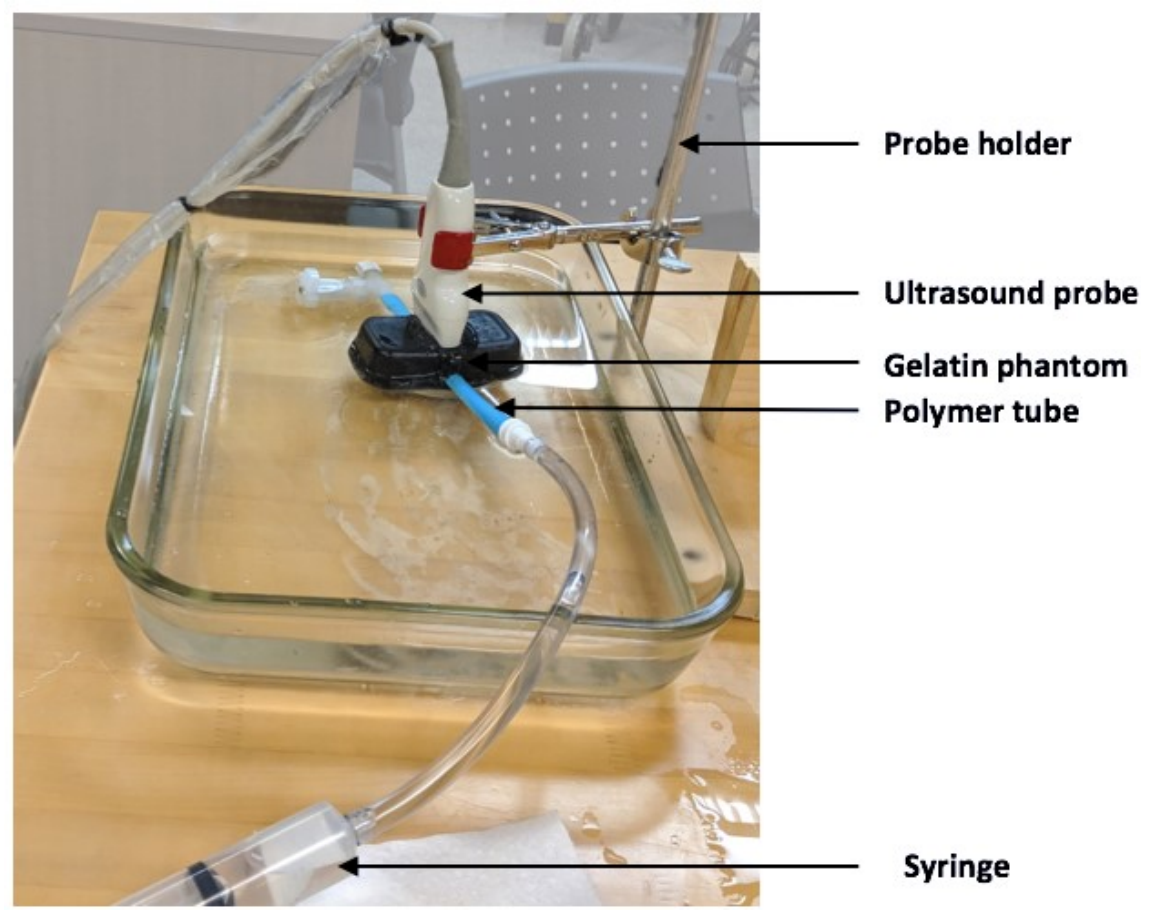

Figure 3.11 Experimental setup for data acquisition of phantom $B$.

The B-mode image of the hydrogel-based phantom and the corresponding motion of the polymer tube simulating the artery wall at the scan line 100 is given in Figure 3.12. It is seen that the graphite particles introduce scattering noise in the B-mode image that is acquired. 


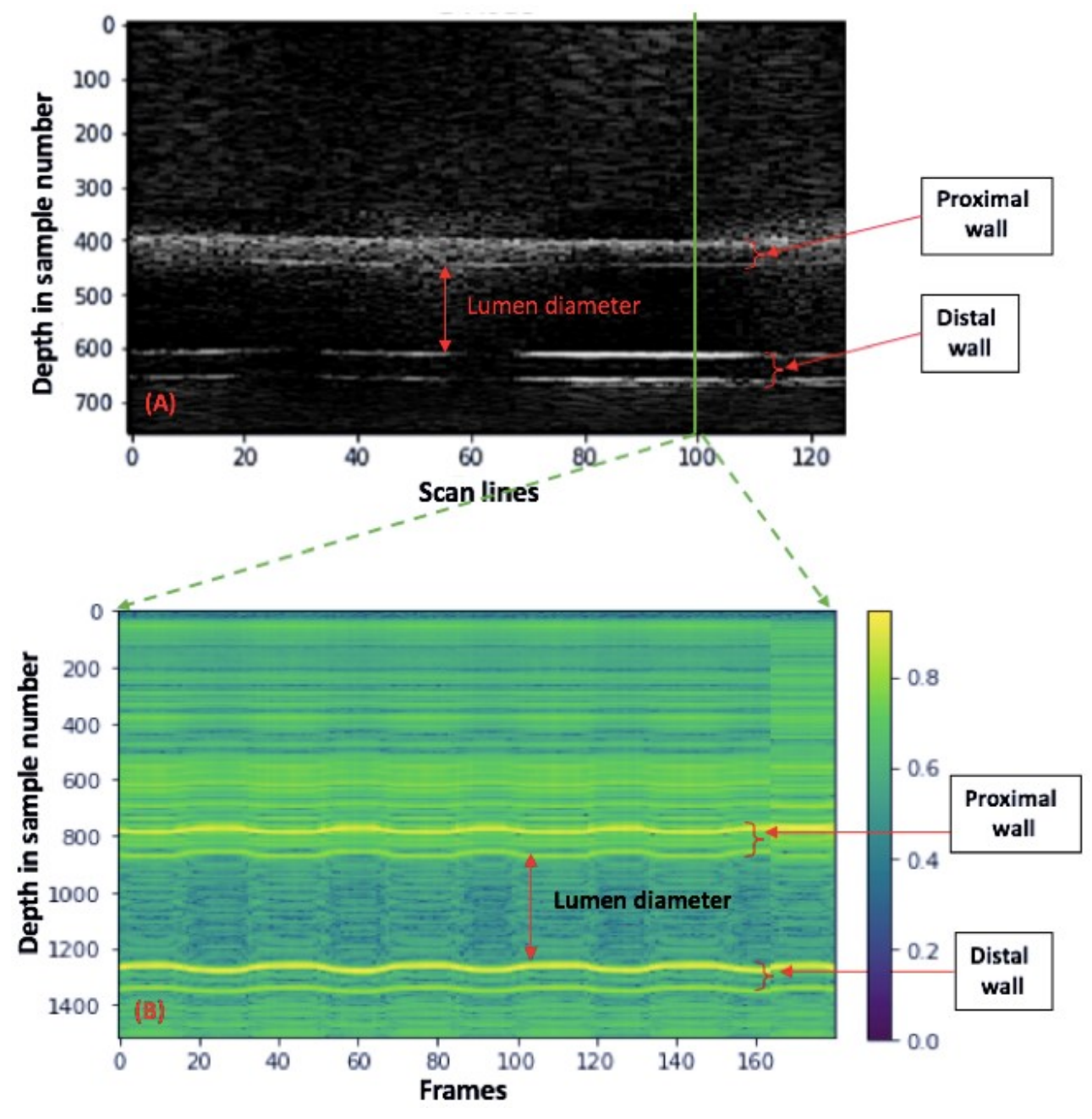

Figure 3.12 B-mode image of Phantom B (A) and the corresponding M-mode image (B) for the scan line \#100.

As explained in Section 3.1.1.2, the sample number representing the depth in the M-mode image is twice as that of the sample number representing the depth in the B-mode signal. It is observed that along with the scattering noises due to the graphite particles in the phantom B, there are artifacts, in which the contrast of the distal walls are missing at the scan lines of around \#30 and \#60. These artifacts are caused probably due to the air bubbles in and around the upper (proximal) wall of the polymer tube. These air bubbles reflect the 
ultrasound and do not allow further transmission into the deeper region. There is also a possibility that at the junction of the hydrogel soft tissue-mimicking material and the polymer tube, there might have been a small amount of a gap filled with fluid or air bubbles. The air bubbles cause the ultrasound signal to be reflected or scattered, causing the noisier region around the proximal walls at around $\# 30$ and $\# 60$ [75]. Along with the motion of the polymer tube walls inside the phantom B, the motion of the surrounding tissuemimicking phantom is also observed in the M-mode image due to the speckle echoes resulted from the scatterers, as shown in Figure 3.12 (b). Later, this is also seen in the case of in-vivo based experiments. The surrounding tissues of the artery also experienced some motion due to the expansion and contraction of the arteries.

\subsubsection{In-Vivo experiments with a human subject}

After evaluating the performance of the proposed algorithm to detect and track the location of the arterial wall in the Phantom A and the phantom B, the algorithm that is performing the best is tested on the ultrasound data acquired from the right common carotid artery of human subjects using the PICUS system and WUS system. The experiment with a human volunteer was approved by the Carleton University ethics board. The ultrasound rf signal from the right common carotid artery of eight human volunteers is collected for this thesis research and is assumed to be the population dataset. It is noted that after assessing the performance of the proposed algorithms with the ultrasound rf signal data acquired with the PICUS clinical ultrasound system, the algorithm that performs the best in the phantom experiments (to be discussed in chapter 5) is selected and used for the in-vivo experiments with the human subjects. The selected algorithm is also tested on the ultrasound rf signals 
signal acquired with the WUS system in M-mode. The in-vivo experimental setups for the acquisition of the ultrasound rf signal at the common carotid artery using the PICUS clinical ultrasound imaging system and the WUS system explained in Section 3.3.1, are given in Figures 3.13 and 3.14, respectively.

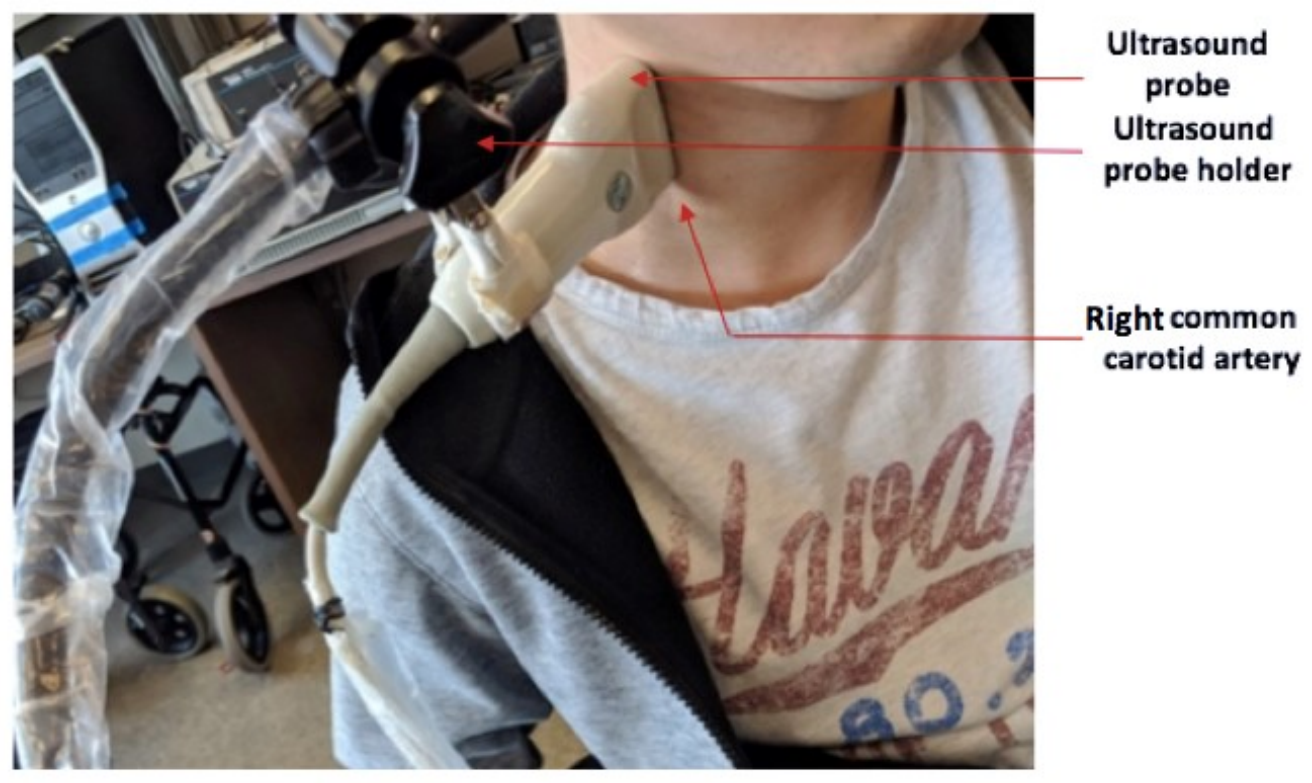

Figure 3.13 Experimental setup for in-vivo data acquisition for the common carotid artery using PICUS clinical ultrasound imaging system with a linear array probe. 


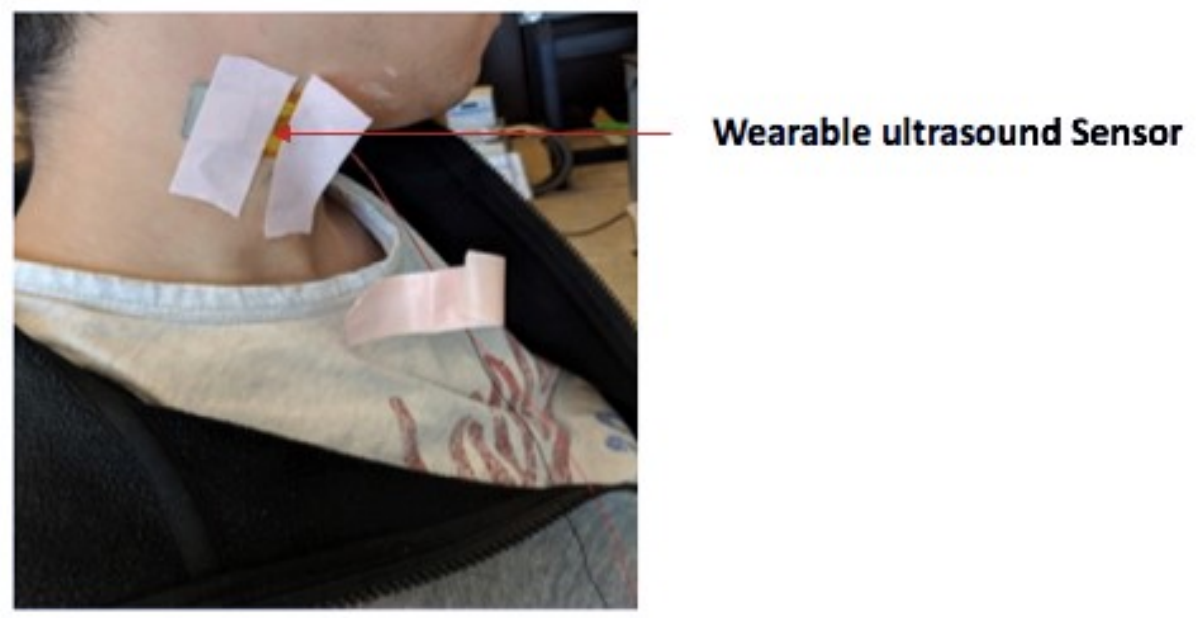

Figure 3.14 Experimental setup for in-vivo data acquisition for the common carotid artery using WUS.

The data acquisition procedure for the first human test subject was guided by a medical practitioner, cardiovascular specialist. The detailed procedure of the in-vivo experiment using PICUS clinical ultrasound imaging system and WUS system as guided by the medical practitioner is given below.

- The human subject is made to rest in a comfortable position, and the right carotid artery pulse is felt by manually by pressing the finger against the larynx.

- The PICUS clinical ultrasound linear probe with the copulant gel is placed transverse to the artery such that the transverse cross-section of the common carotid artery is observed in the B-mode image. The PICUS clinical ultrasound probe is adjusted in such a way that the center of the common carotid artery appears in the middle of the B-mode image, in which the center of the carotid artery is beneath the middle of the linear probe. 
- The mid-point position of the transducer over the right common carotid artery is marked using a marker, and the probe is rotated to align itself along with the longitudinal position of the right common carotid artery.

- The PICUS clinical ultrasound is now fixed using the probe holder at a chosen desired position, and the B-mode image and the corresponding ultrasound rf signals are acquired for 6 seconds using the PICUS clinical ultrasound imaging system. This procedure is repeated three times for every human subject to obtain enough data to evaluate the proposed algorithms of automatic artery detection and diameter tracking (to be discussed in Chapter 4).

- After acquiring the ultrasound rf signals using the PICUS clinical ultrasound system, the WUS system is used to acquire the ultrasound rf signal at the same location as the right common carotid artery of the human subjects. The WUS is attached with the ultrasound copulant gel and fixed using an adhesive tape at the same position, which was marked in the in-vivo experiment with the PICUS system).

- Ultrasound rf signals were acquired for about 12 seconds in each M-mode acquisition using the WUS is obtained. The acquisition condition of the WUS system was explained in Section 3.1.2.2.

The B-mode image of the common carotid artery of a human subject acquired using the PICUS clinical ultrasound system and the ultrasound rf signal M-mode image of the artery wall corresponding to the B-mode scan line $\# 80$ are given in Figure 3.15 (A) and (B), respectively. 


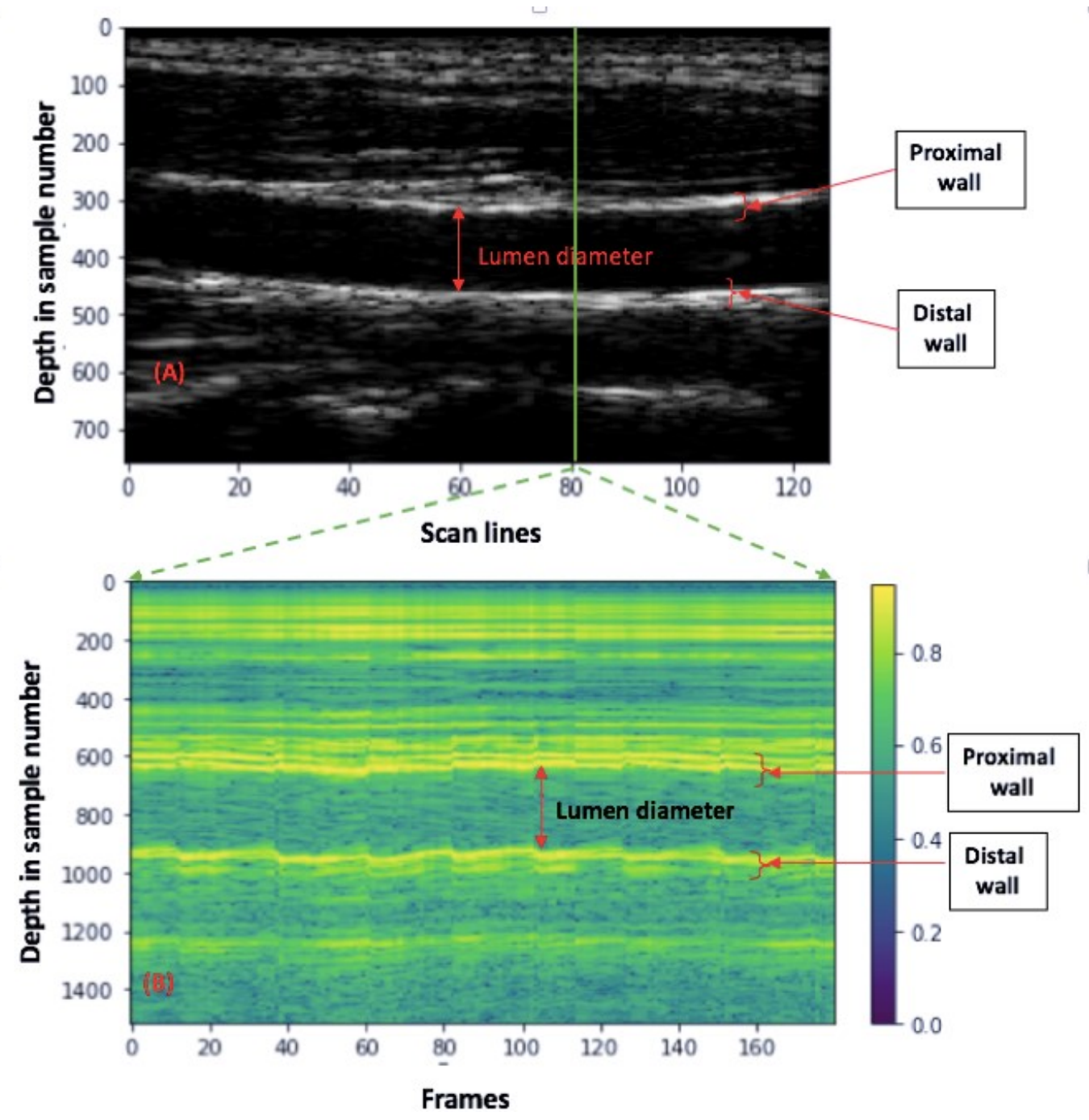

Figure 3.15 B-mode image (A) of in-vivo experiment and the corresponding ultrasound rf signal M-mode image (B) at the Bmode scan line $\# \mathbf{8 0}$.

By comparing the B-mode images of the in-vivo experiment with that of Phantom B in Figure 3.12 (A), it is seen that the tissue-mimicking phantom exhibited the similar noisy signal observed in the in-vivo B-mode image. Further, with the help of the guidance provided by a medical practitioner, the artery walls are located in the B-mode image and the M-mode image. It is easier to identify the artery wall in a B-mode image once the 
corresponding M-mode signal at a scan line of interest is observed because the motion of the artery wall due to the blood flow is evident in the M-mode rf signal image. It is noted that this feature of artery wall motion can be used as a feature to locate the presence of the artery proximal and distal walls in the M-mode rf ultrasound signal. Figure 3.16 presents the M-mode ultrasound rf signal image of the right common carotid artery of a human subject acquired with the WUS system. The opposite motion of the artery proximal and distal walls is observed.

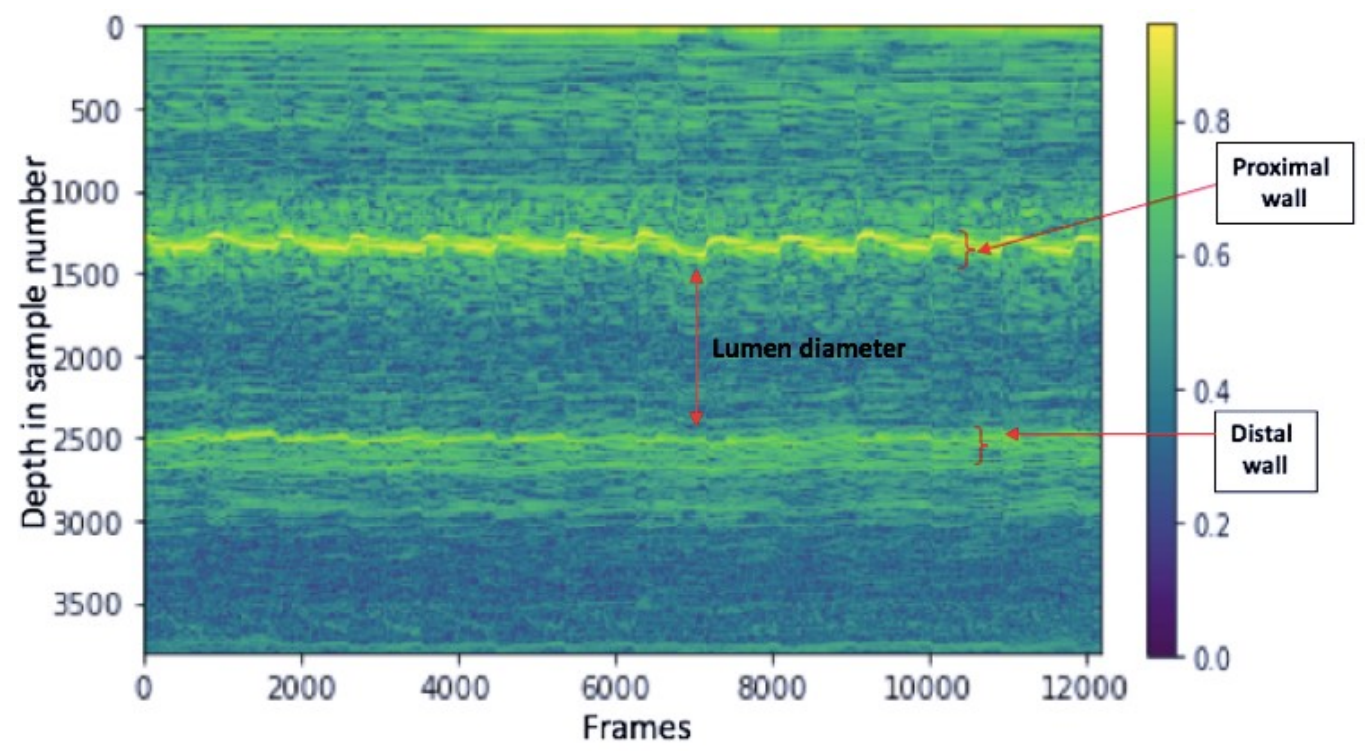

Figure 3.16 M-mode ultrasound rf signal image of the right common carotid artery of a human subject acquired with the WUS system.

The distal wall observed in Figure 3.16 is faint as compared to the proximal wall. It is because the ultrasound signal energy generated by the WUS composed of a signal UT made of a PVDF piezoelectric polymer is smaller than that generated by the clinical ultrasound 
imaging probe composed of array UTs made of PZT piezoelectric ceramic [73]. The developed algorithm is also tested on the ultrasound rf signal acquired with the WUS system. The result is also compared with that of the PICUS clinical ultrasound system. 


\section{Chapter 4: Algorithm development for artery region detection and lumen diameter tracking}

In this chapter, the algorithm to perform the task of automatic artery wall detection and lumen diameter tracking is explained. As discussed in Section 2.2.6, artery lumen diameter tracking using the M-mode ultrasound image by a single UT is an efficient and costeffective method. The flowchart explaining the process of artery diameter detection using M-mode ultrasound rf signal is given in Figure 4.1. 

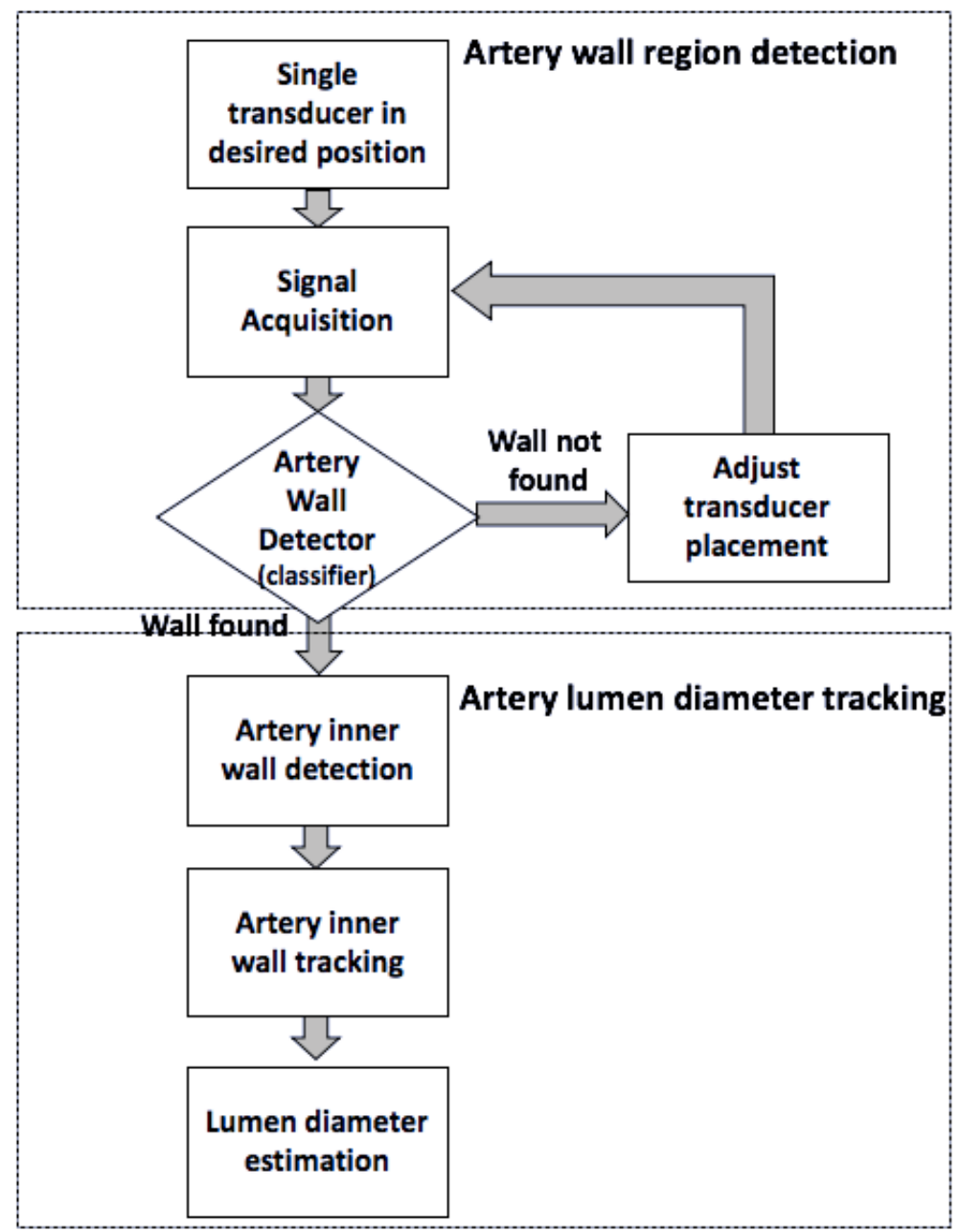

\section{Figure 4.1 Flowchart of artery wall detection and lumen diameter tracking process using M-mode ultrasound rf signal.}

In the case of B-mode imaging, the ultrasound image of tissues and organs is visible in the display monitor, and this helps the operator to adjust and place the transducer exactly over the artery, which is the region of interest. However, in the case of an M-mode ultrasound rf signal from a single UT, the operator cannot be sure if the UT is placed over the artery for the ultrasound signal acquisition. Hence, the proposed algorithm is designed to assist the operator by automatically detecting the region containing the artery for tracking the 
change in the artery lumen diameter during a cardiac cycle. The algorithm proposed to track the lumen diameter using an M-mode image is, therefore, subdivided into two sections: artery wall region detection and lumen diameter tracking. This chapter explains the development of a methodology for the artery wall region detection and lumen diameter tracking.

This chapter is organized in the following manner. A machine learning-based classifier is proposed in this thesis to detect the region in M-mode image that contains the artery wall in Section 4.1. The process of creating a ground truth, extracting features, and designing a group of classifiers to evaluate the performance is explained in Section 4.1. The classifiers are evaluated based on their overall performance in different environmental conditions (Phantom A, Phantom B and in-vivo human subject), as explained in Section 3.2. After evaluating the performance of different classifiers, the classifier with the best performance is then used as the classifier (or detector) for arterial wall region selection. Once the classifier identifies the region containing the artery wall, the technique to detect the inner wall of the artery and track the lumen diameter during the cardiac cycle is explained in Section 4.2.

\subsection{Artery wall region detector}

The artery wall region is a subset of the M-mode image formed by the acquired rf signal of the wall echoes from the UT. The artery wall that is closer to the UT is referred to as proximal wall (PW), while the farther one is referred to as distal wall (DW) henceforth. 


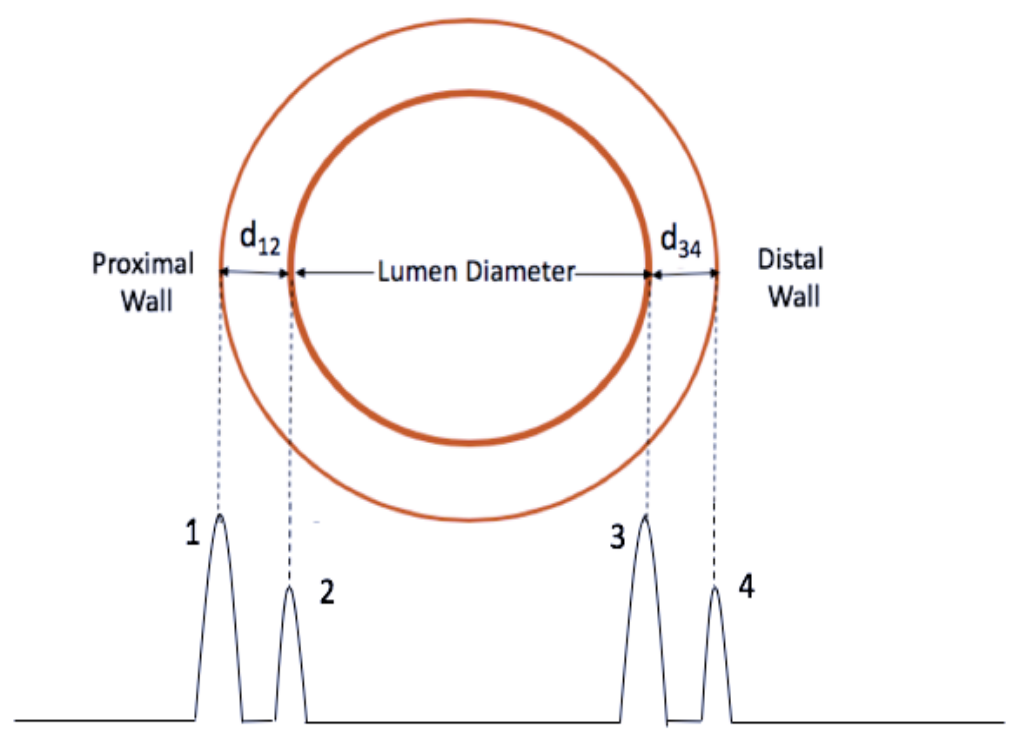

Figure 4.2 Conceptual sketch of the common carotid artery and expected ultrasound echo peaks indicating the locations of the artery walls.

Figure 4.2 shows the conceptual sketch of the ultrasound wall echoes from the common carotid artery in A-mode signal. The ultrasound travels through soft tissues and fluids, and it also reflects at the tissue boundary surfaces depending on the echogenicity of the tissues. The artery walls create an echo in the ultrasound rf signals which are called wall echoes. The wall echoes 1 and 2, shown in Figure 4.2, corresponds to the proximal outer and proximal inner echoes, respectively, while those 3 and 4 correspond to distal inner and distal outer echoes. The proximal outer and inner walls together are called as proximal wall, while the distal outer and inner walls together are called as distal wall. The $\mathrm{d}_{12}$ and $\mathrm{d}_{34}$ correspond to the thicknesses of the proximal and distal artery walls, respectively. The distance between the echoes 2 and 3 is the lumen diameter of the artery. The process of 
segmenting and labeling a subsection of the M-mode image is called ground truth creation and is explained in the following section.

\subsubsection{Ground truth creation of the artery wall region by manual segmentation}

The B-mode image acquisition system contains a display that guides the operator to place the ultrasound probe over the artery. In the case of using a single element UT, there is no display available to confirm the placement of the sensor or transducer over the common carotid artery. Hence, a classifier is trained to provide confirmation. As explained in Section 2.1.1, the M-mode signal is acquired with UT over the artery. The machine learning based-classifier then segments the subset of the M-mode signal into a region containing either proximal wall, distal wall or no boundary. Since a supervised machine learning approach is used in this thesis, a classifier is trained with the labeled subsets of the M-mode signal. To obtain the labeled subsets and to better evaluate the performance of the classifier, it is necessary to have data from different acquisition conditions and locations. This is done with the help of the B-mode image obtained by the PICUS clinical ultrasound imaging system, which consists of 128 equally spaced A-mode scan lines, as explained in Figure 2.2. Hence, the B-Mode signal obtained by the PICUS clinical ultrasound probe, placed longitudinal direction to the common carotid artery, contains 128 A-mode signals, at the different regions of the artery. To manually locate the region containing the artery wall in the ultrasound rf-signal, a subset of the ultrasound rf signal containing the artery wall should be specified by the observer. The observer defines the location of the artery wall region by specifying the range of the depth (sample number) in the ultrasound rf signal. By specifying the range along depth (sample number), a subset of the ultrasound $\mathrm{rf}$ signal 
containing the artery wall echo is selected. The size of the subset is decided based on factors such as the thickness of the artery wall, artery wall motions during the cardiac cycle, and the tissues surrounding the artery wall. Since the subset is to be manually specified for all the A-mode scan lines obtained using the PICUS clinical ultrasound probe, an algorithm to manually specify the region containing the artery wall is explained in the flow chart given in Figure 4.3.

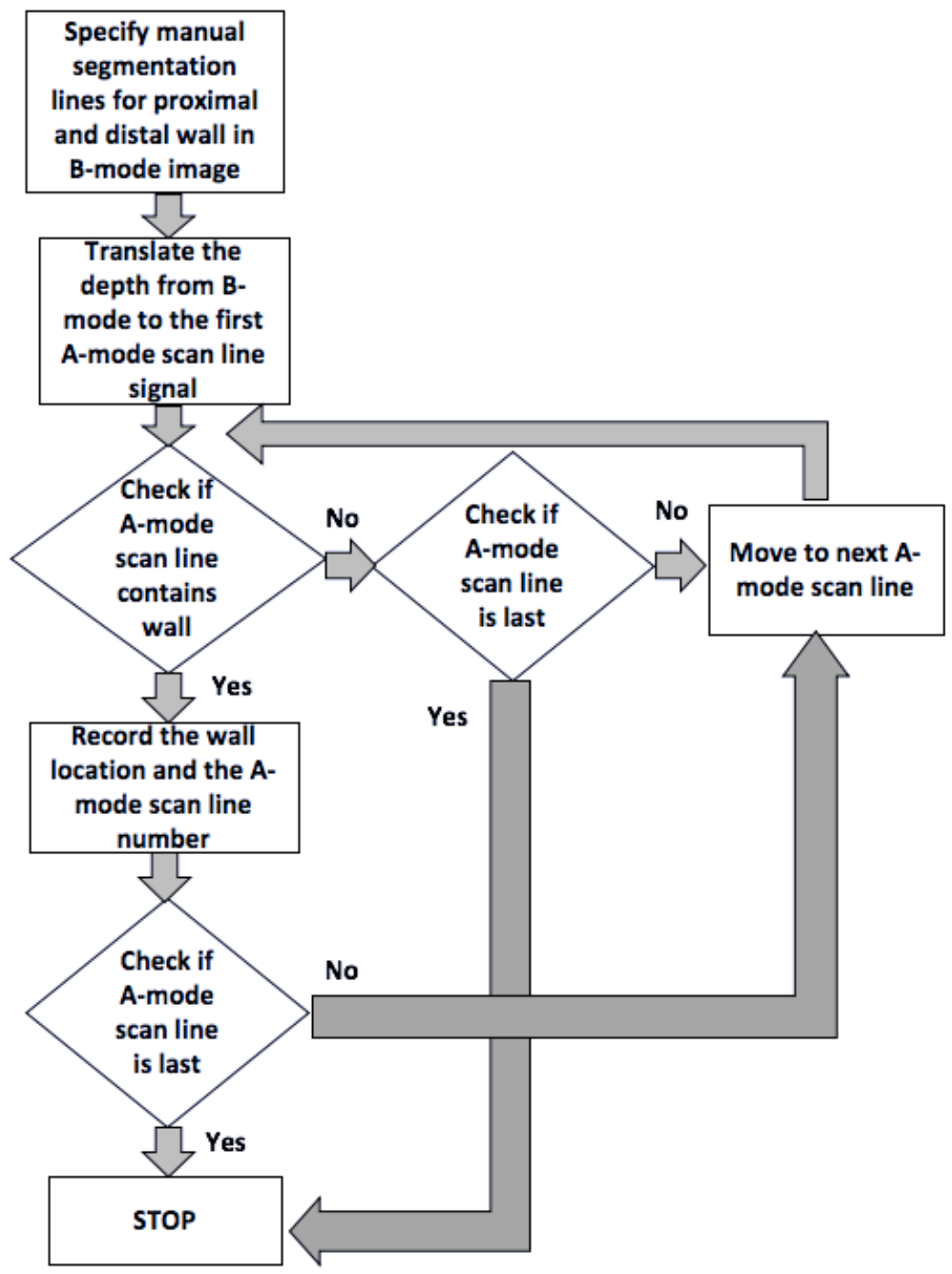

Figure 4.3 Flowchart of the A-mode scan line segmentation

process. 
Based on the observation from a B-mode image, the region containing the artery wall is manually specified to be within a certain depth. The lines (orange lines) in Figure 4.4, specifying the region, along with the depth, are called as manual segmentation lines. The upper manual segmentation lines specify the range of depth in which the proximal wall of the artery is observed, while the lower manual segmentation line gives the range of depth in which the distal wall is observed. Figure 4.4 and Figure 4.5 shows the manual segmentation line being specified in the B-mode image of the water-based phantom A and how it translates to specifying a subset of ultrasound rf signal containing the artery wall.

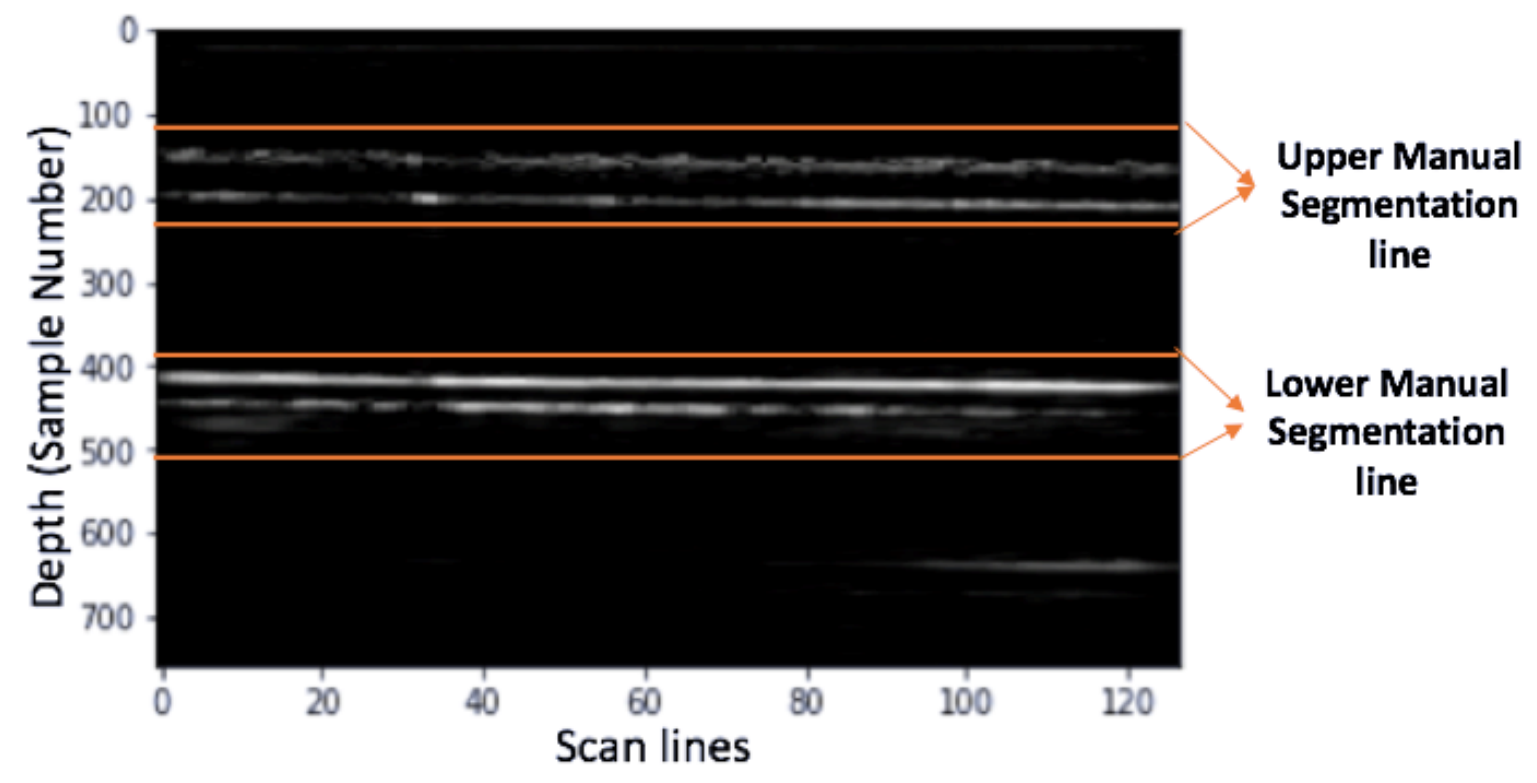

Figure 4.4 Manual selection of the artery wall region in a B -mode signal with water-based phantom A.

The upper and lower manual segmentation lines are manually specified by defining the range of the depth (sample number) in the B-mode signal. The manual segmentation line 
in the B-mode image translates to specifying a subset of the ultrasound rf signal containing either of the artery walls, for all the 128 A-mode scan lines. The peak of the ultrasound rf signal echo observed in the subset is located with the help of a simple peak detection technique [82] on its envelope. The envelope of the acquired ultrasound rf signal $x[i]$ is given as $e[i]$, calculated as given in the Equations (4.1) and (4.2).

$$
e[i]=\sqrt{x[i]^{2}+D H T\{x[i]\}^{2}}
$$

where $\operatorname{DHT}\{x[i]\}$ is the Discrete Hilbert transform $x[i]$ given by the following Equation (4.2).

$$
\operatorname{DHT}\{x[i]\}=\left\{\begin{array}{l}
\frac{2}{\pi} \sum_{m=o d d} \frac{x[m]}{i-m}, m \text { is even } \\
\frac{2}{\pi} \sum_{m=\text { even }} \frac{x[m]}{i-m}, m \text { is odd }
\end{array}\right.
$$

The ultrasound rf signal obtained is noramilized to an amplitude range from - 1 to 1 . Hence, the envelope $e[i]$ is normalized to an amplitude range from 0 to +1 . The envelope of the ultrasound $\mathrm{rf}$ signal is called as the A-mode signal. The location of the peak of the first wall echo obtained within the upper manual segmentation lines corresponds to the location of the proximal outer wall, while the location of the peak of the first wall echo obtained within the lower manual segmentation line corresponds to the distal inner wall. Subsets of the rf ultrasound signal containing the proximal wall echoes and distal wall echoes, respectively, 
are obtained using a one-dimensional window of fixed length called as a gate. The size of the gate $\left(G_{\text {size }}\right)$, for the phantom and the in-vivo experiments are based on the thickness of the artery walls and the artery wall motions due to the cardiac cycle. It is also essential to make sure a portion of the tissue surrounding the artery and blood inside the artery is included in the gate as they can be used as a feature to locate the artery wall. As per the design considerations of the algorithm proposed, the value of the gate length in the sample number should be a multiple number of five. After observing the thickness of the artery wall [6], [9], [50], maximum distension due to the cardiac cycle and the speed of the ultrasound in the human tissue, a gate size of $4.62 \mathrm{~mm}$ (200 sample points) is chosen for the case of the in-vivo based experiments with the PICUS clinical ultrasound imaging system.

Similarly based on, the thickness of the polymer tube, the maximum distension of the tube caused due to the manual pumping and the speed of the ultrasound in the Phantoms A and $\mathrm{B}$, a gate size of $4.5 \mathrm{~mm}$ (200 sample points) and $5 \mathrm{~mm}$ (200 sample points) is chosen, respectively. In case of the human in-vivo signals from the WUS system, a gate size of $4.93 \mathrm{~mm}$ ( 800 sample points) is chosen. The echoes (proximal wall echoes and distal wall echoes) in the upper and lower manual segmentation, respectively, are found with the help of a simple peak detection technique as given in [82]. The location of proximal outer wall echo (in case of upper manual segmentation line) and distal inner wall echo (in case of lower manual segmentation line) are identified by locating the first wall echoes observed within the segmentation lines respectively. The location of the first echo observed within the manual segmentation line is considered as the reference point and the starting and 
ending point of the gate (one-dimensional window) around the first echo (reference point) is shown in Figure 4.4 and Figure 4.5.

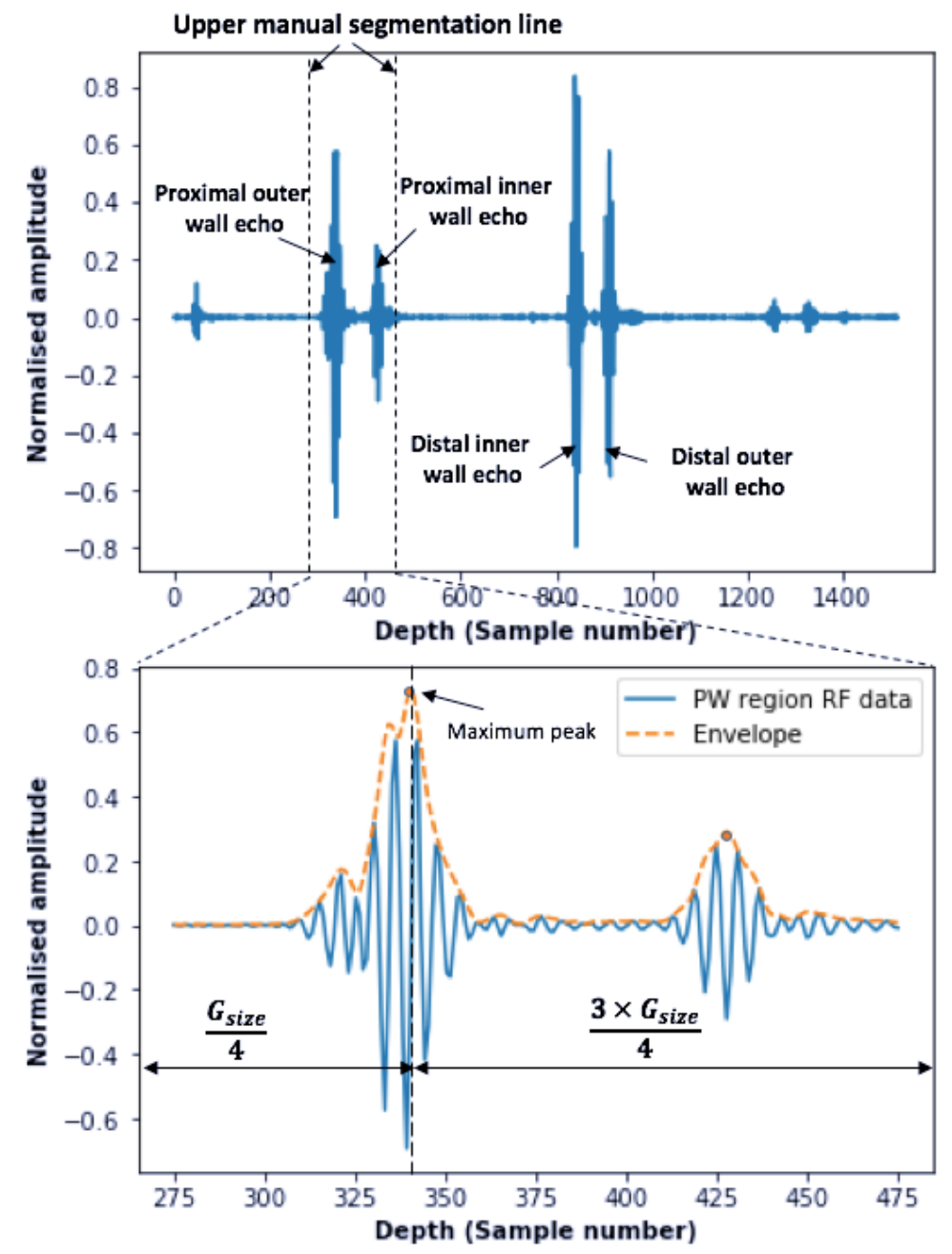

Figure 4.5 Gate selection of artery wall region in the A-mode signal.

In Figure 4.5, the location of the wall echo (maximum peak in A-mode signal) of proximal outer wall echo is considered as the reference point, and the boundary of the gate is 
specified. The starting point of the gate is at a distance of $\left(\frac{G_{\text {size }}}{4}\right)$ sample numbers to the left and the endpoint of the gate is at a distance of $\left(\frac{3 \times G_{\text {size }}}{4}\right)$ sample numbers to the right from the echo peak. The same procedure is repeated to select the gate around the distal wall.

After selecting the subsets (by placing the gates around the walls) containing the proximal wall and distal wall, the artery characteristic to expand and contract during the cardiac cycle is used to verify if the peaks of the echoes selected belong to the artery walls and not the stationary tissues. Since there is a consistent motion of the artery walls due to the cardiac cycle, the echoes from the artery walls shift their position. On examining the wall echo of the rf signals acquired over a period of time, it can be seen that the echoes corresponding to the proximal and distal walls of the artery move opposite direction to each other in a periodic manner. Therefore, such wall motions could be used as a feature to verify if the echoes belong to the proximal and distal artery walls.

In order to track the echoes of the proximal and the distal wall, let $P W_{t}[i]$ and $D W_{t}[i]$ be considered as the segment of the ultrasound rf signal containing the proximal and the distal wall, respectively, at a time instance t. The location of echo peak of the proximal outer and distal inner wall at is obtained using the simple peak detection method and is denoted by $i_{\text {pow }}$ and $i_{\text {diw }}$. The location of the distal inner and proximal outer wall at the time instance $t+1$ is given by Equations (4.3) and (4.4). 


$$
\begin{aligned}
& D W_{t+1}\left[i_{\text {diw }}\right]=D W_{t}\left[i_{\text {diw }}+\tau_{d w}[t]\right] \\
& P W_{t+1}\left[i_{\text {pow }}\right]=P W_{t}\left[i_{\text {pow }}+\tau_{p w}[t]\right]
\end{aligned}
$$

where $\tau_{p w}$ and $\tau_{d w}$ are the shifts of the echo peak in the proximal wall, and distal wall echoes, respectively, due to a cardiac cycle. These shifts are estimated by calculating the location of the maximum of the autocorrelation between the subset of the ultrasound $\mathrm{rf}$ signal (obtained using gate) containing the proximal wall acquired at the time instance $t$ and $t+1$. Similarly, the shifts of the distal wall is obtained by obtaining locating the maximum of the autocorrelaion between the subset of the ultrasound rf signal (obtained using gate) containing the distal wall acquired at the time instance $t$ and $t+1$ The shifts are obtained using the Equation (4.5) \& (4.6)

$$
\begin{aligned}
& \tau_{d w}[t]=\operatorname{argmax}\left(R_{D W_{t}, D W_{t+1}}\right) \\
& \tau_{p w}[t]=\operatorname{argmax}\left(R_{P W_{t}, P W_{t+1}}\right)
\end{aligned}
$$

where $\mathrm{R}$ is the autocorrelation between two $\mathrm{rf}$ signals denoted by $D W_{t}$ and $D W_{t+1}$, respectively, for the distal wall, and those of $P W_{t}$ and $P W_{t+1}$, respectively, for the proximal wall. The location of the maximum value of the $\mathrm{R}$ gives the amount of the shift, $\tau$, of the wall echo peak. The autocorrelation between two real discrete signals two rf signals $D W_{t}$ and $D W_{t+1}$ is given by Equation (4.7), while the autocorrelation between $P W_{t}$ and $P W_{t+1}$ is given by Equation (4.8). 


$$
\begin{aligned}
& R_{D W_{t}, D W_{t+1}}[i]=\sum_{m=-\infty}^{\infty} D W_{t}[m] D W_{t+1}[m-i] \\
& R_{P W_{t}, P W_{t+1}}[i]=\sum_{m=-\infty}^{\infty} P W_{t}[m] P W_{t+1}[m-i]
\end{aligned}
$$

The Pearson coefficient $(r)$, which measures the linear relationship between the two datasets is used to identify the relationship between the proximal and the distal wall distension. Since the proximal and the distal wall of the artery are moving in the opposite direction to each other, the Pearson correlation coefficient of the distension of the proximal outer wall and distal inner wall should be negative [20], [83]. The distension of the proximal outer and the distal inner artery wall is given by $\tau_{d w}[t]$ and $\tau_{p w}[t]$, and the Pearson correlation coefficient between the distension is calculated using Equation (4.9) .

$$
r=\frac{\sum_{t=1}^{n}\left(\tau_{p w}[t]-\overline{\tau_{p w}}\right)\left(\tau_{d w}[t]-\overline{\tau_{d w}}\right)}{\sqrt{\sum_{t=1}^{n}\left(\tau_{p w}[t]-\overline{\tau_{p w}}\right)^{2}\left(\tau_{d w}[t]-\overline{\tau_{d w}}\right)^{2}}}
$$

The motion profiles of the proxima and distal wall echoes, whose Pearson correlation coefficient is negative, is used for ground truth creation. A two-dimensional window of fixed size is slid over the M-mode signal to obtain a smaller subset of the M-mode signal. The subsets obtained using the two-dimensional window are called segmented windows and are classified into one of the three categories of proximal, distal, or no boundary (no wall region). Since the location of the artery wall region for a selected A-mode scan line is known, the segmented windows can be labeled into one of the three categories of the 
proximal wall, distal wall, or no boundary. Figure 4.6 shows the segmented window in an M-mode signal.

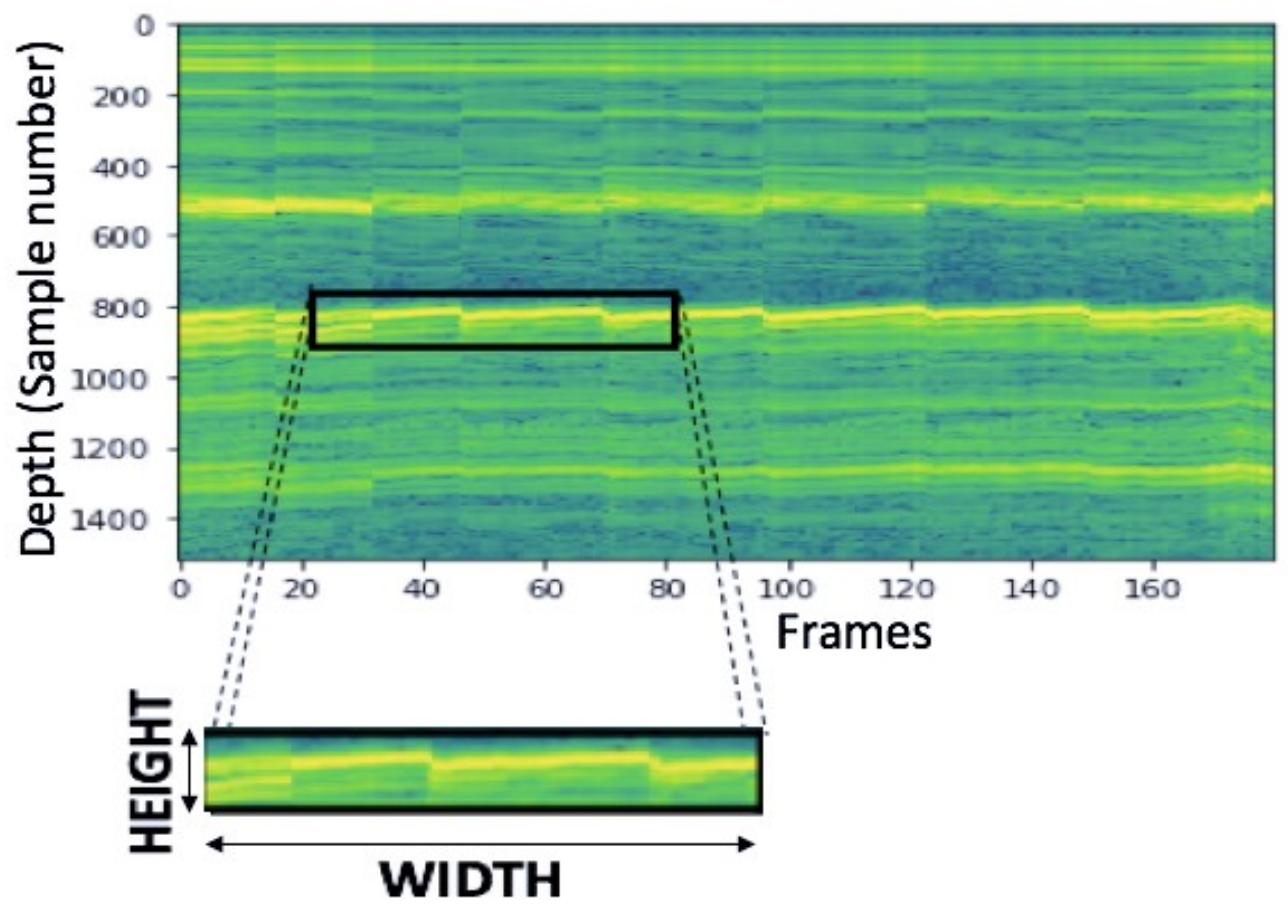

Figure 4.6 Segmented window in an M-mode signal.

Since the M-mode signal is a collection of the A-mode signals along time, by design, the gate length translates to the height of the segmented window. The width of the window is selected to capture the temporal dynamics of the cardiac artery cycle. As explained in Section 2.1.1, the pulse repetition rate or frame rate of the PICUS clinical ultrasound system used in this thesis is $30 \mathrm{~Hz}$. A width of 30 frames captures at least one complete cardiac cycle, given that the heart rate is above 60 beats per minute (bpm). In the case of the WUS system, the frame rate is $1000 \mathrm{~Hz}$, and a width of $1000 \mathrm{~Hz}$ captures at least one 
complete cardiac cycle. Hence, the width of the segmented window for the case of the PICUS clinical ultrasound system is chosen 60 frames, and in the case WUS system, it is 2000 frames, in order to have at least two cardiac cycles in the window. Similarly, the depth of the segmented in case of the signal acquired from the WUS system is 800 sample points (i.e., With $125 \mathrm{MHz}$ sampling rate: $1540 \mathrm{~m} / \mathrm{s}$ x $8 \mathrm{~ns} \times 800 / 2=4.928 \mathrm{~mm}$ )as it covers the artery wall thickness and the artery wall motion range during the cardiac cycle. The size of the segmented window in the case of the M-mode signal with the WUS system is of the size 800 sample points (window height) $\times 2000$ frames (window width) and the size of the segmetned window for M-mode signal with PICUS clinical ultrasound system is 200 sample points (window height) $\times 60$ frames (window width). In order to create a set of labeled segmented windows to train the classifier, a segmented window is obtained for every ( $\frac{\text { window height }}{5}$ ) sample points along the fast-time domain and every frame along the frames of the M-mode signal. The stride length of ( $\frac{\text { window height }}{5}$ ) sample points or an overlap of $20 \%$ is selected experimentally to strike a balance between creating a diverse set of data and the computational resource available. Since the region containing the artery walls for every A-mode scan line is already known using the technique mentioned above, the segmented window containing either of the walls is labeled as a proximal wall or distal wall, respectively. While the remaining segmented windows, which do not contain the artery wall, are labeled as no boundary. The labeled segmented windows that are obtained using this method are the ground truth for the classifier. 


\subsubsection{Feature Extraction for automatic wall region detection}

The segmented window which is a two-dimensional matrix, has columns that represent the depth and rows that represent the frames. Features are extracted along both the columns (depth) and rows (frames) of the segmented window. The features obtained along fast-time are called the fast-time features, and the features obtained along the columns, i.e., frames, are called the slow-time features. It is to be noted that only the time domain features are considered for the feature extraction of the segmented windows since it is computationally faster to calculate than the frequency domain features. The main aim of the feature extraction is to capture the pattern of the data observed in the segmented window.

\subsubsection{Fast-time features}

The features that are obtained by selecting the columns, i.e., along the depth of the segmented window, are called the fast-time features. The columns of the matrix representing the segmented window contains a segment of the ultrasound rf signal along the depth. The segmented window containing the proximal and the distal wall contains the rf signal of the wall echoes reflected by the artery walls, while the segmented window classified as the no wall regions may not contain rf signal of the wall echoes. In this section, the fast-time features of the rf signal along the depth including energy, maximum amplitude and minimum amplitude will be discussed.

\section{Signal power along fast-time}

The signal power obtained along the columns of the segmented window represents the power of the signal along the depth. The signal power obtained is used as a fast-time feature 
for the classifier. Let, $\mathrm{X}(\mathrm{i}, \mathrm{z})$ be the segmented window of the M-mode signal. The average signal power along the fast-time for the segmented window selected is given by the Equation (4.10).

$$
E=\frac{\sum_{f=1}^{M} \sum_{d=1}^{N}|X(i, z)|^{2}}{M \times N}
$$

where $\mathrm{N}$ is the height of the segmented window (gate length), and $\mathrm{M}$ is the width of the segmented window (number of A-mode scan line frames in a segmented window). If the segmented window contains the region of the artery wall in them, there is a wall echo observed in the rf signal of the segmented region. The signal power obtained in the segment containing the echo is higher than the segment without the echo. Since this is a fast-time feature, the signal power along the columns, i.e., the average signal power of the acquired signal along the depth for all the frames in the segmented window, is considered as fasttime signal power of the segmented window. The distribution of the fast-time signal power across the various labeled segmented windows for the case of Phantom A, Phantom B, and the in-vivo experiments are given in Figure 4.7, which is a kernel density estimation of the histogram values. 
(A)
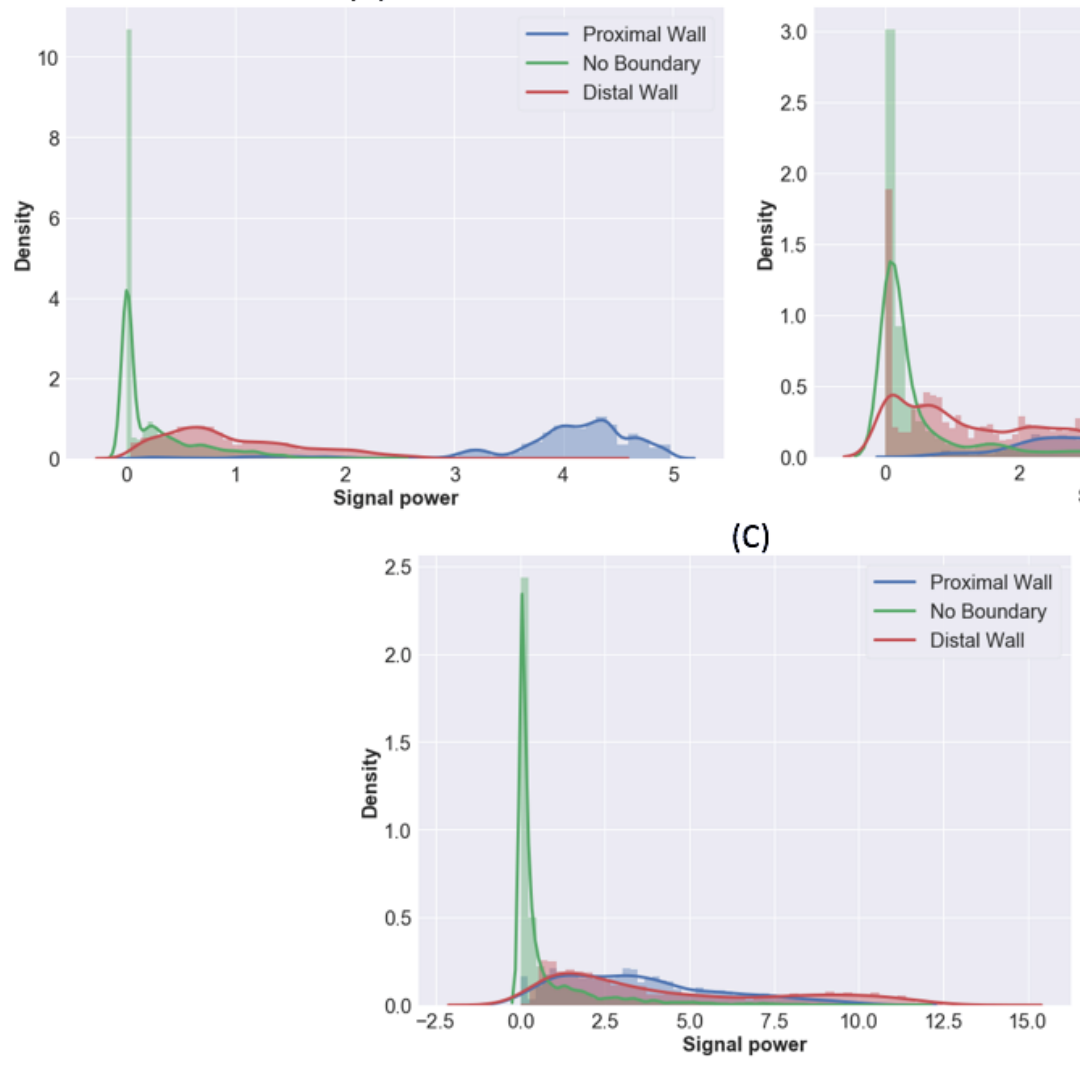

(B)

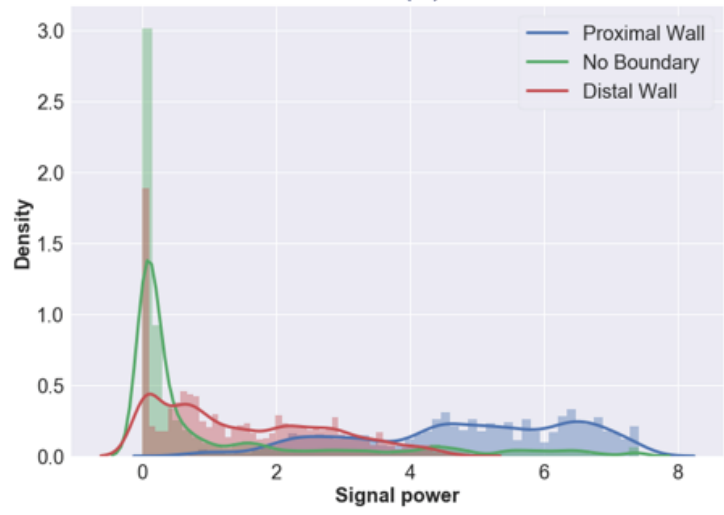

Figure 4.7 Distribution of signal power along fast-time for (A)

Phantom A, (B) Phantom B, and (C) In-vivo.

On observing the distribution of the fast-time signal power for all the three cases, it is seen that the signal power for different segmented windows are not distinct, as there is an overlap in the distribution of signal power for different segmented windows. This energybased feature along the fast-time domain shows the need for more features to improve the classification performance of the classifiers. There is an overlap between the no boundary and the wall region, observed in the case of the Phantom A, due to the bounce back, as explained in Section 3.2.1.2. In the case of Phantom B and the in-vivo experiments, the overlap is due to the scattering noise and other tissues in the no-boundary region. 


\section{Maximum amplitude along the fast-time domain.}

The maximum amplitude along the fast-time domain translates to the peak detection technique applied to the A-mode $\mathrm{rf}$ signal $x[i]$ to locate the boundary echoes. The maximum amplitude along the fast-time domain is obtained by selecting the maximum value along the columns (i.e., along depth) of the segmented window. In the segmented window, the columns represent the fast-time domain of the signal. The average of the maximum value along the columns of the segmented window of ultrasound rf signals represents the maximum amplitude along the fast-time domain for the given segmented window. If there is a presence of wall in the segmented window region, then due to the echo produced by the wall, the maximum amplitude in the segment is higher as compared to the rest of the regions. 
(A)

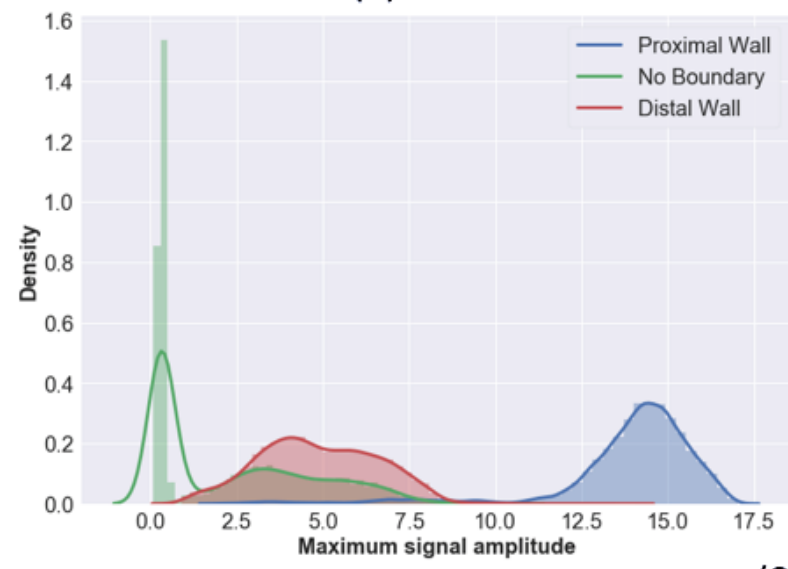

(C)
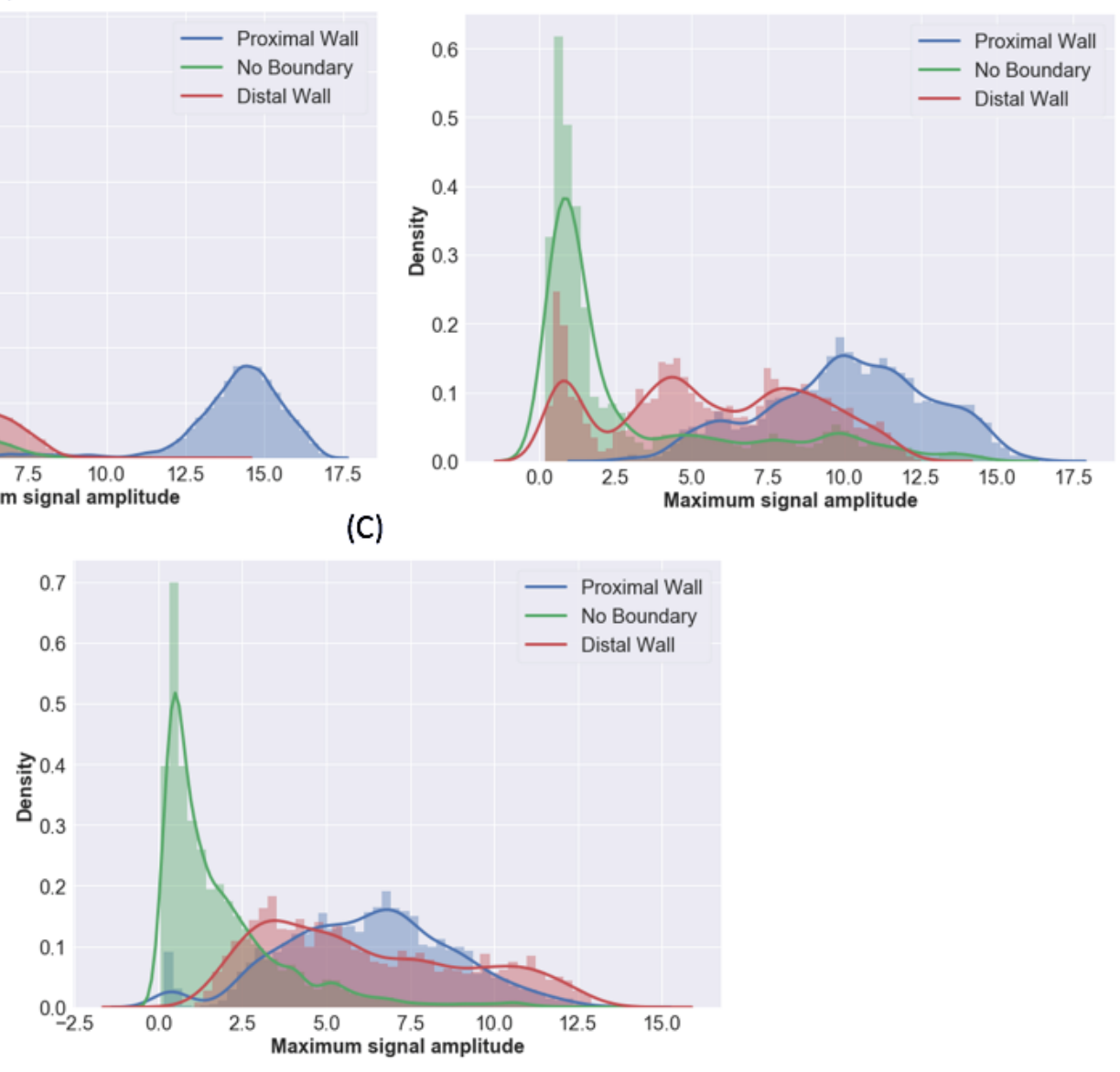

Figure 4.8 Distribution of maximum amplitude along fast-time for

(A) Phantom A, (B) Phantom B, (C) In-vivo.

From Figure 4.8, it is observed that in the case of the Phantom A, the maximum amplitude that is observed in the segmented window containing the wall has a higher value of amplitude than the region that does not include the artery wall. But it is not the case for the Phantom B and in-vivo experiments. It is because of the fact that higher signal amplitudes are also observed on the echoes created by the scattering noises in the stationary tissues. 


\section{Minimum amplitude of the negative peaks of rf-signals.}

Similar to the previous feature that is observed, the minimum value of the columns of the segmented window represents the minimum value in the region of depth covered by the segmented window. It is obtained by the average of the minimum amplitude value along the columns of the segmented window containing the ultrasound rf signals. If there is a presence of wall in the segmented window region, then due to the echo produced by the wall, the minimum value in the segment is lower than the rest of the areas. Since the segmented window contains a collection of ultrasound rf signals, the minimum value of the region containing the proximal wall and distal obtained should be comparatively lower than the minimum value obtained in the region containing no wall. The distribution of the values obtained for the labeled segmented windows is shown in Figure 4.9. 
(A)

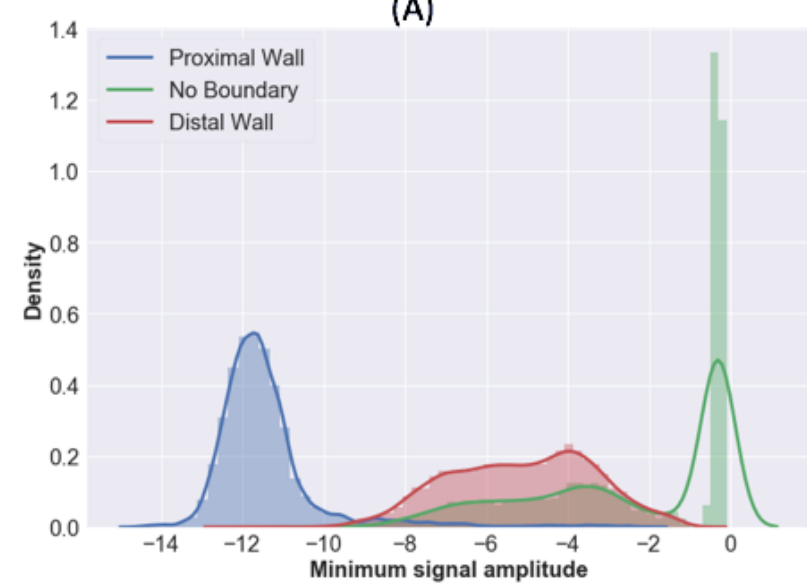

(B)

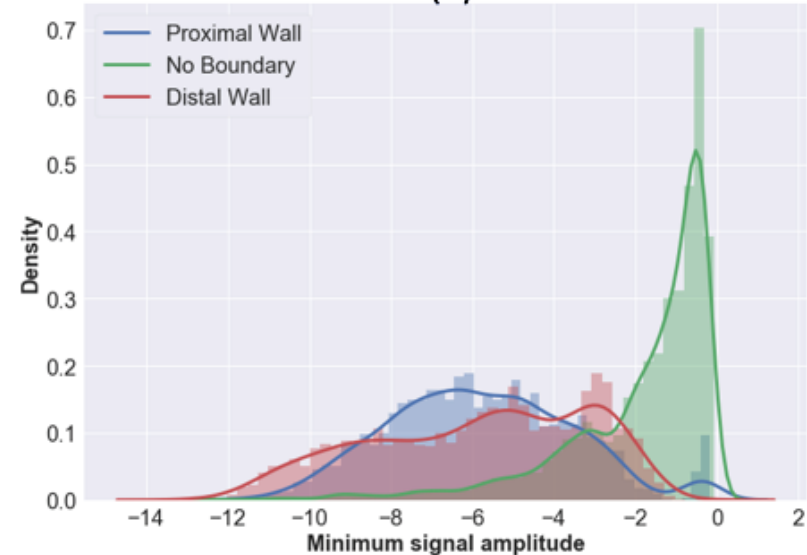

(C)

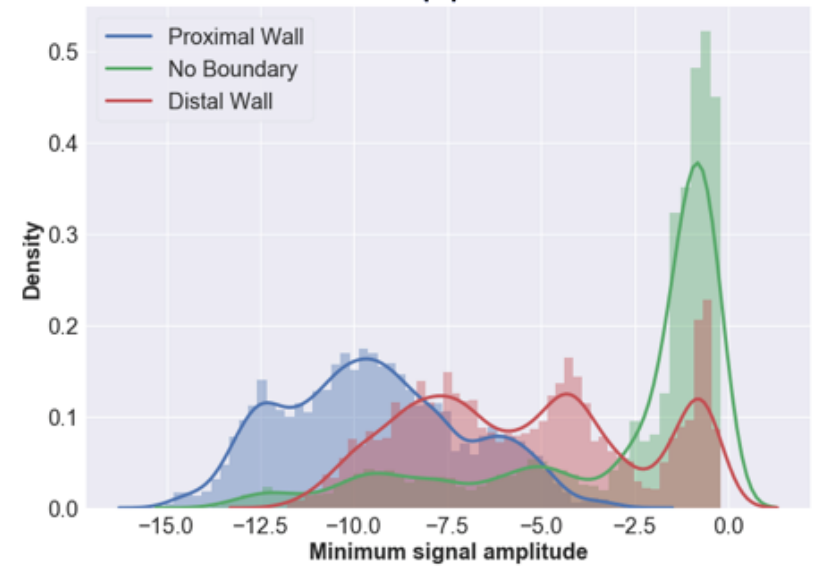

Figure 4.9 Distribution of minimum amplitude along fast-time for

\section{(A) Phantom A, (B) Phantom B, (C) In-vivo.}

In Figure 4.9, it is seen that the distribution of the minimum amplitude value along fasttime for different segmented windows with the wall is different from that of the one without an artery wall in it. In the case of the phantom-based experiments, there is an overlap between the distribution of the values obtained for the region of no boundary and artery walls. In the case of Phantom A, bounce backs are observed in the rf signals which creates an echo in the region of no artery walls. This bounce backs as explained in Section 3.2.1.2, attributes to the overlap in the distribution of minimum amplitude value along fast-time for 
the segmented windows containing walls and no boundaries. In the case of the gelatinebased tissue-mimicking phantom, there are echoes observed due to the scattering particles in the phantom, which causes an overlap in the distribution. In the case of the in-vivo based experiments, the overlap in the distribution is because of scattering echoes from the stationary tissues around the artery wall.

\subsubsection{Slow-time features}

The features that are obtained along the rows of the segmented windows are called as the slow-time features. Each value in a row corresponds to the value of the ultrasound rf signal obtained along the frames for a fixed depth length. The slow-time features that are considered in this thesis research are maximum amplitude, minimum amplitude and fractal dimension, along the slow-time domain. Since the slow-time features are along the rows of the segmented window, a feature is calculated for each row in the segmented window. Each row in the segmented window can be considered as a time series, as it represents the values of the ultrasound rf signals for a fixed depth length over a fast time period. The number of rows in the segmented window (height) is selected as per the conditions explained in Section 4.1.1. As per the conditions, the height of the segmented window is selected, such that it includes the artery wall thickness, its motion due to cardiac cycle and tissue around the artery and blood in the artery lumen. Since the segmented window also contains a part of the surrounding tissue along with the artery wall, it is sub-divided into five equal subsections $\left(\mathrm{DR}_{1}, \mathrm{DR} 2, \mathrm{DR} 3, \mathrm{DR} 4\right.$ and $\left.\mathrm{DR}_{5}\right)$ along the depth. The slow segmented windows are divided into five subsections because the height of a subsection is approximately equal to the thickness of the artery wall considered. Each subsection

contains $I$ data points of the $\mathrm{rf}$ signal, where $I$ is given by $\left(\frac{\text { Height of the segmented window }}{5}\right)$. 
The height of the segmented window, as discussed in Section 4.1.1, is 200 sample points for the signal acquired using PICUS clinical ultrasound system. Hence, the size of each sub-section (DR) is of size 40 sample points. The average of the feature obtained in each chosen subsection is then considered to represent the slow-time feature in the subsection. Thus, for every slow-time feature, we have five values representing the five subsections of the segmented window. Thus, the average of the feature value obtained for each subsection can determine if the artery wall exists in the subsection.

\section{Maximum amplitude}

The echoes in the acquired rf signal along the fast-time domain may be resulted from artery wall boundaries and/or scatterers in the tissues. Due to this, obtaining the maximum amplitude to detect the presence of the artery wall might be misleading. Thus, it would be helpful to also include the features along the slow-time domain to differentiate peaks from artery wall echoes of interest from those from the undesired echoes, in order to improve the reliability and accuracy of the proposed algorithm of artery wall detection. The maximum amplitude value in each row of the segmented window (time series), formed by the subset of the ultrasound rf signal, is considered as a slow-time feature. Since the artery walls are moving along slow-time domain, depending on the movement of the artery walls, the maximum amplitude observed in the subsections of a segmented window will vary. The maximum amplitude of each subsection in a segmented window is calculated by averaging the maximum amplitude values of all the rows in each subsection. In Figure 4.10 (A) and (B), it is observed that the maximum amplitude value of the subsection with artery walls is higher than that of the region without artery walls. 

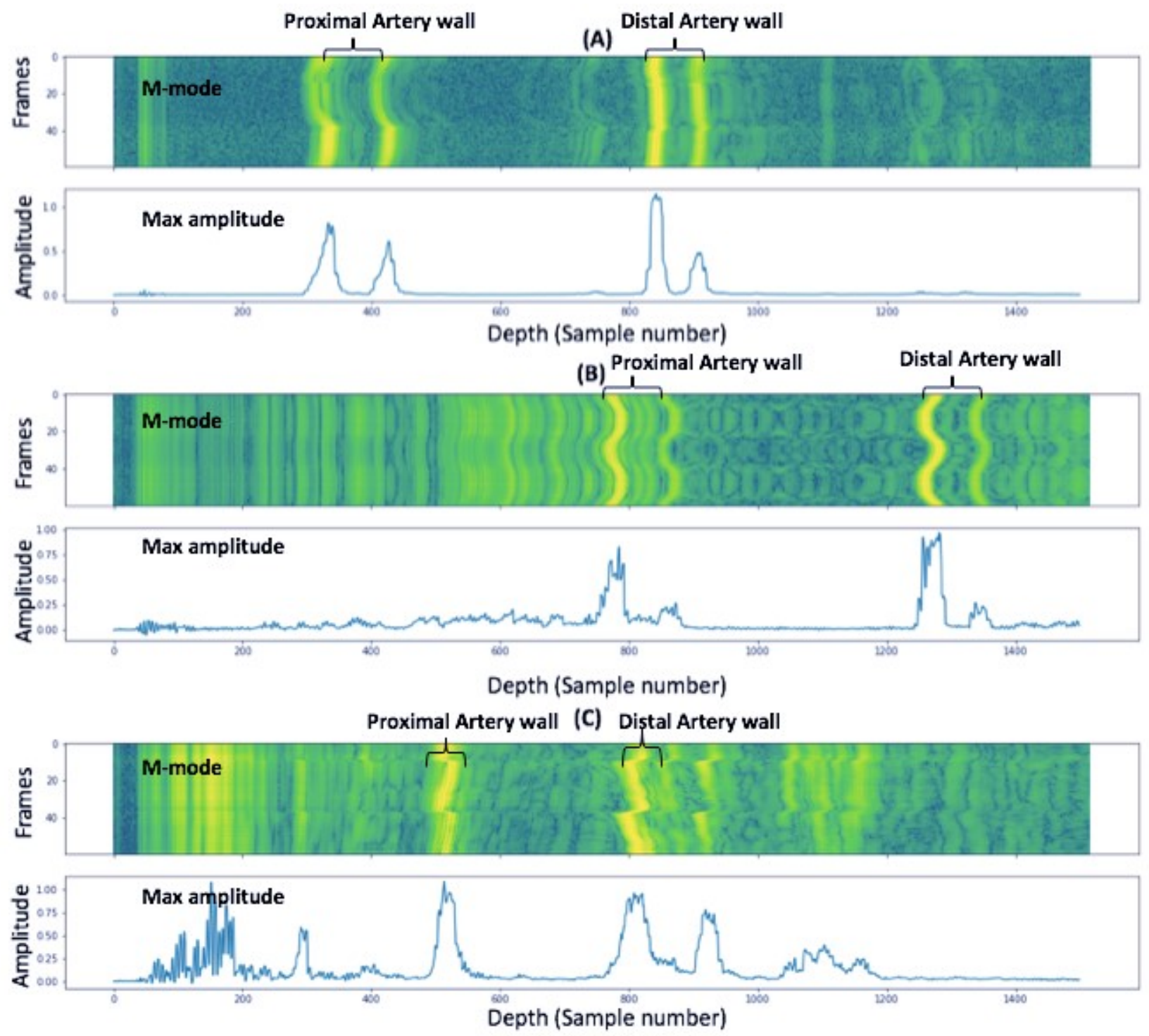

Figure 4.10 M-mode signal and the corresponding maximum amplitude along slow-time for Phantom $\mathrm{A}(\mathrm{A})$, Phantom $\mathrm{B}(\mathrm{B})$, and in-vivo (C).

Figure 4.10 shows how the maximum amplitude is distributed along the frames when the artery walls undergo a quasi-periodic movement. Further, it is observed in Figure 4.10 (C) that the region that contains the stationary tissues at the depths of 0-300 and 1000-1200 has 
a high amplitude observed along the slow-time region as well, but is different from the one of the moving tissues as the distribution of the maximum amplitude along the depth in the case of the moving tissues is more wider as compared to that of the stationary tissues. Thus, for a selected segmented window, there are $\mathrm{N}$ values of maximum amplitude along slowtime. These $\mathrm{N}$ values are averaged into five values, each representing a subsection. The distribution of the maximum amplitude along the slow-time is given by the five values obtained by averaging the echo amplitude in each of the five sub-divided segments (from DR1 to DR5) of the segmented window. The results are presented in Figures 4.11-4.15, respectively. 
(A)
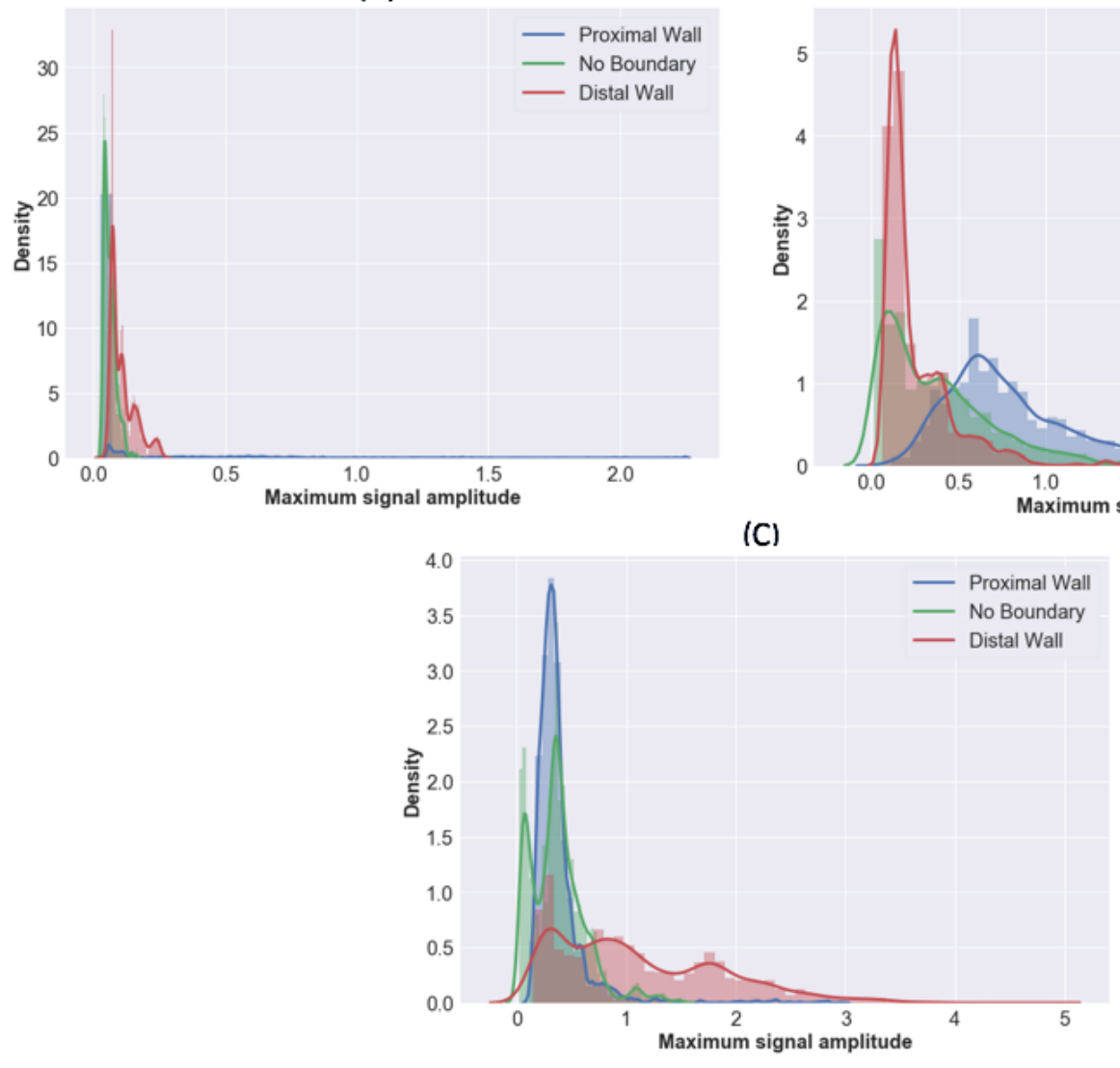

(B)

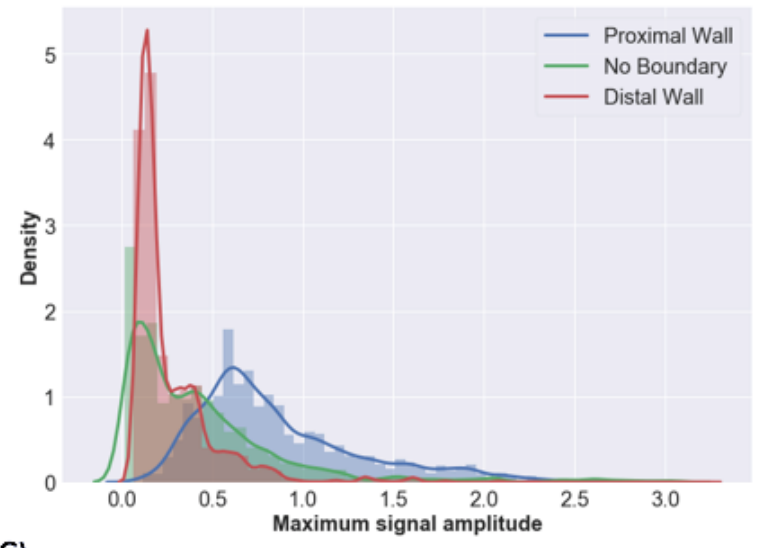


(A)

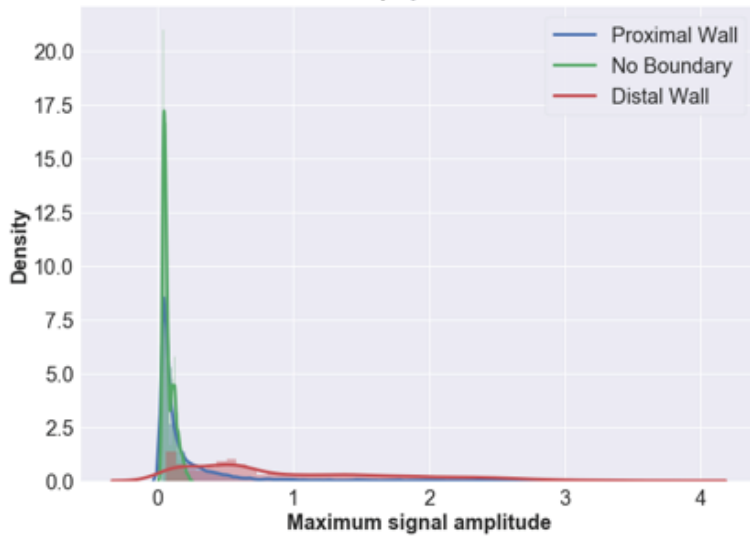

(B)

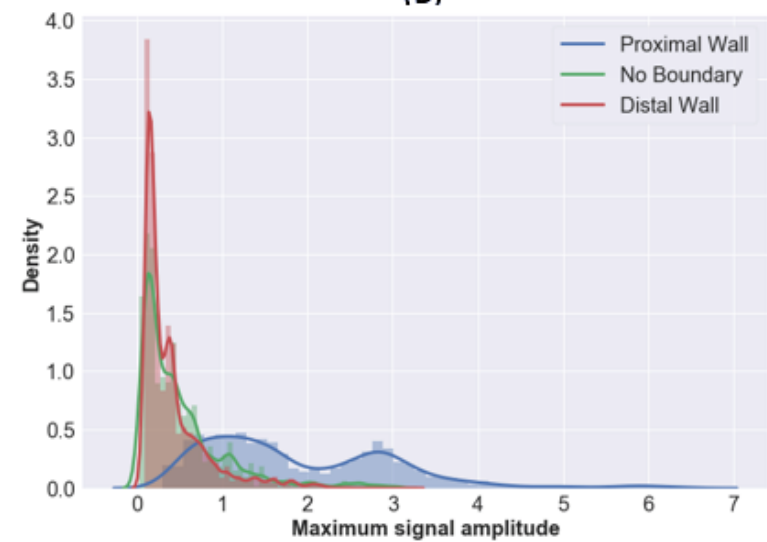

(C)

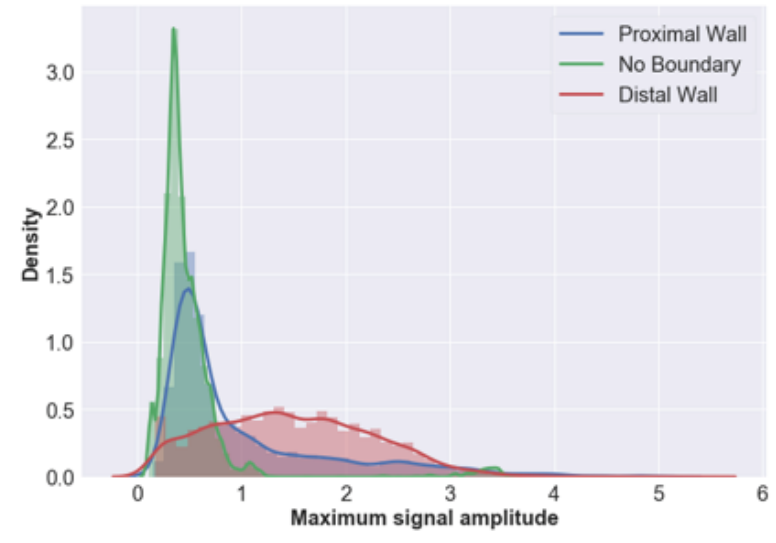

Figure 4.12 Distribution of maximum amplitude values of

$D R_{2}$ along slow-time for (A) Phantom A, (B) Phantom B, (C) Invivo. 
(A)
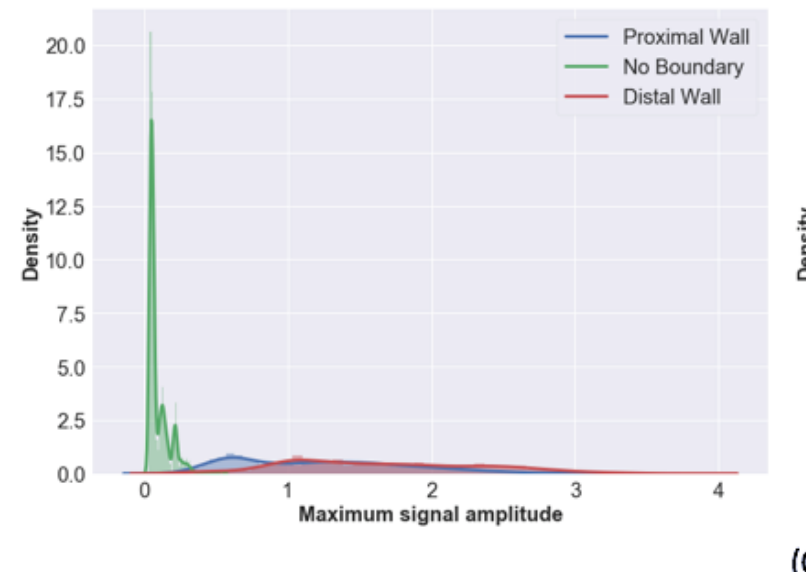

(B)

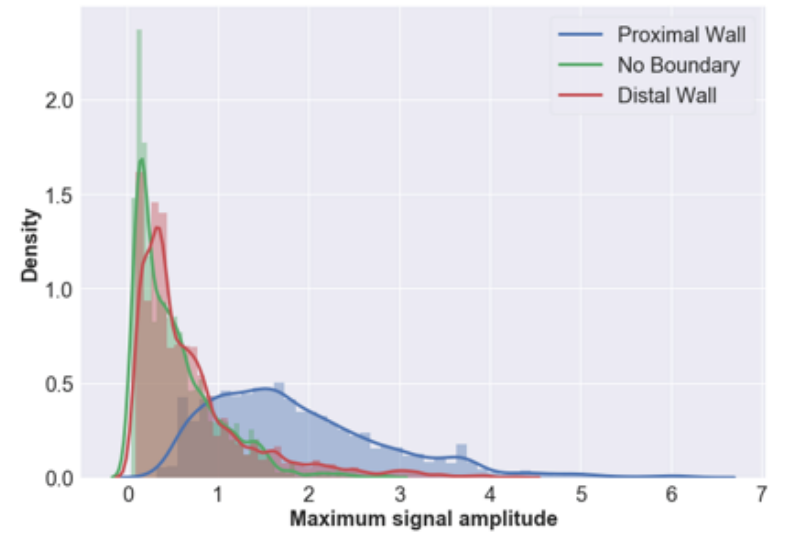

(C)

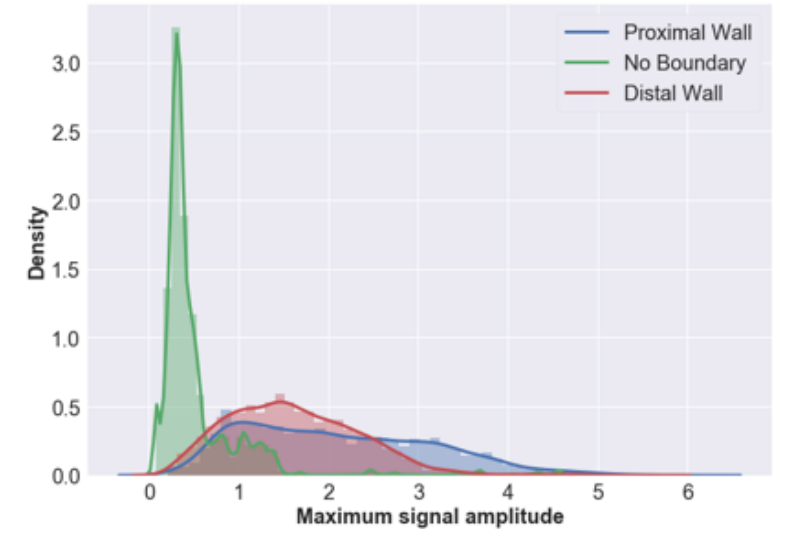

Figure 4.13 Distribution of maximum amplitude values of

$D R_{3}$ along slow-time for (A) Phantom A, (B) Phantom B, (C) In-

vivo. 
(A)

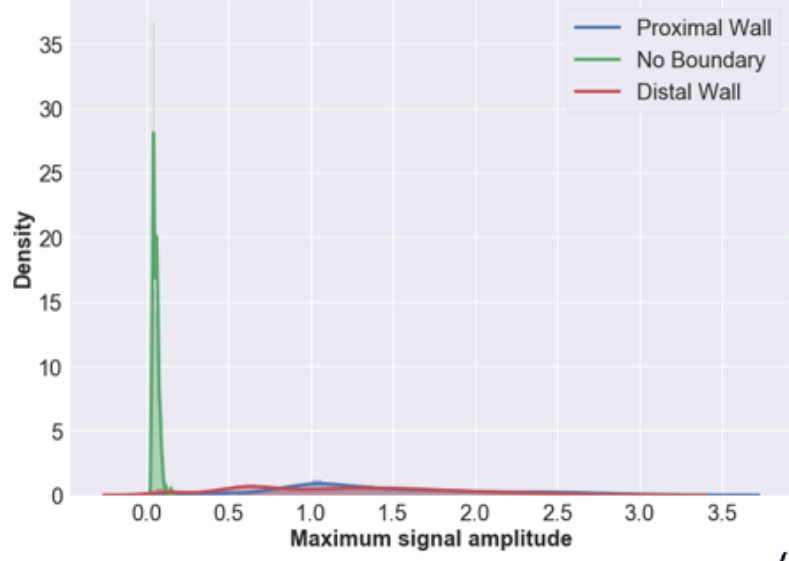

(B)

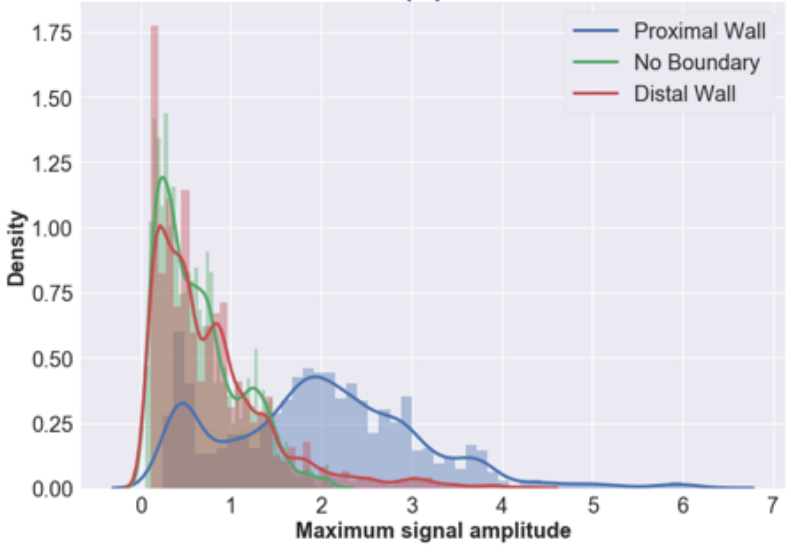

(C)

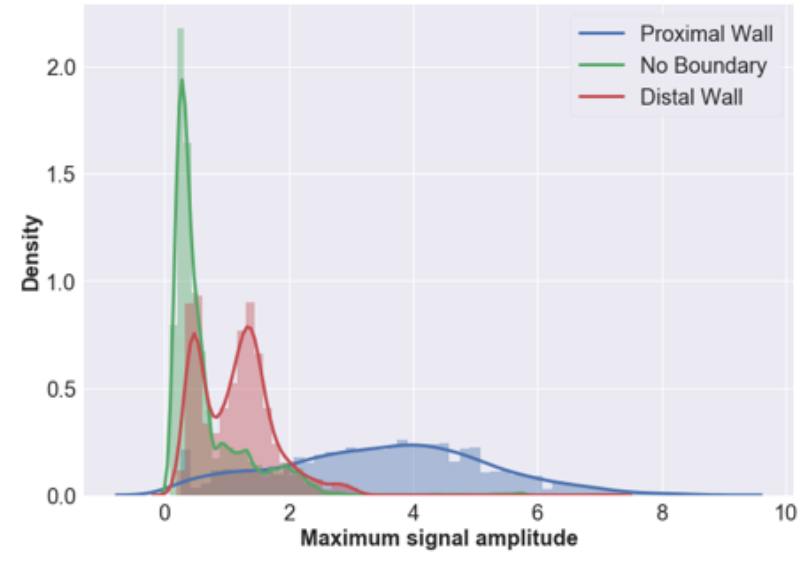

Figure 4.14 Distribution of maximum amplitude values of

$D R_{4}$ along slow-time for (A) Phantom A, (B) Phantom B, (C) Invivo. 
(A)
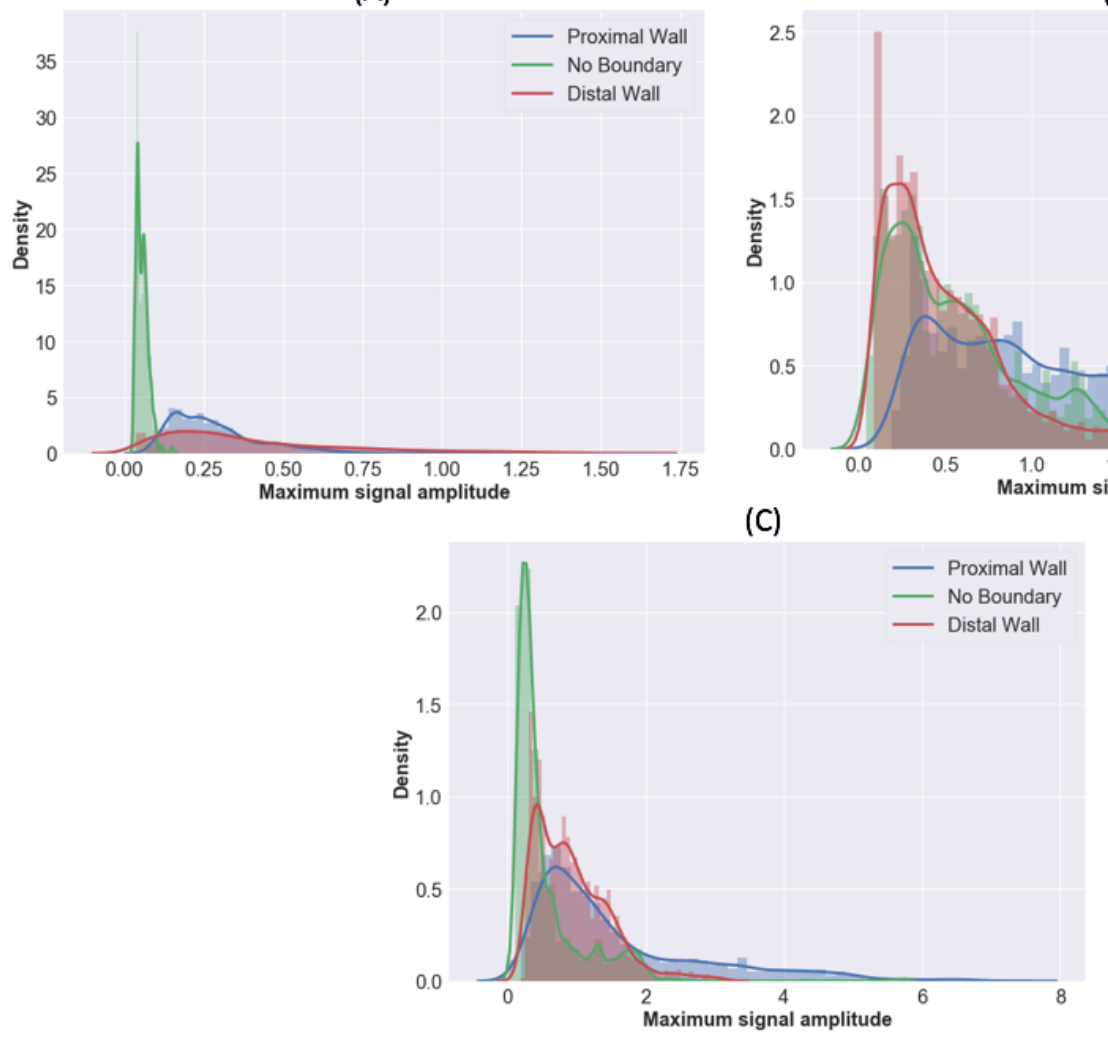

(B)

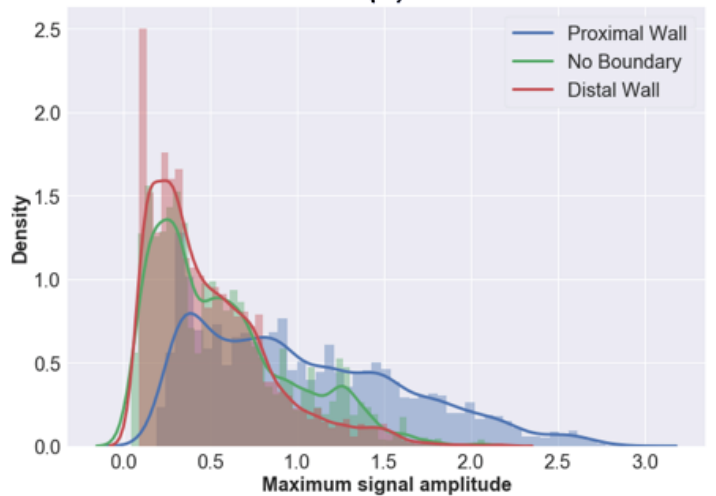

(C)

Figure 4.15 Distribution of maximum amplitude values of

\section{$D R_{5}$ along slow-time for (A) Phantom A, (B) Phantom B, (C) In-}

vivo.

It is observed in Figure 4.11 (B) (C)-Figure 4.15 (B) (C) that the averaged maximum amplitude of the rf ultrasound signal along the slow-time domain for all the three categories (proximal artery wall, distal artery wall, and no artery wall boundary) of the segmented window is overlapping with each other for the Phantom B and in-vivo subject. The overlap is because the segmented windows labeled as no boundary regions also contain echoes due to the scattering noises from the stationary tissues in the case of the Phantom B and in-vivo experiments. On observing Figure 4.11 (A) - Figure 4.15 (A), it is clear that the maximum amplitude values observed in the subsection regions DR3 and DR4 has no overlap between 
the no boundary region and wall region which can be used as a feature to classify the segmented windows.

\section{Minimum amplitude}

Similar to the feature of the maximum value of the ultrasound rf signal along the slow-time domain, the minimum value along the slow-time domain captures the artery wall as shown in Figure 4.16. 

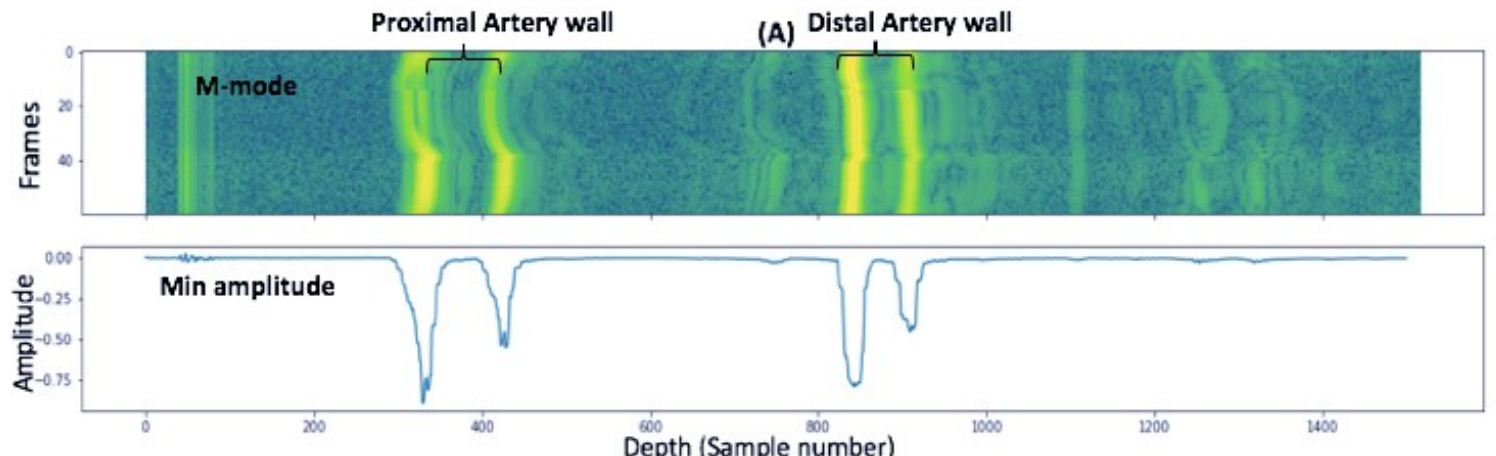

(B) Proximal Artery wall

Distal Artery wall
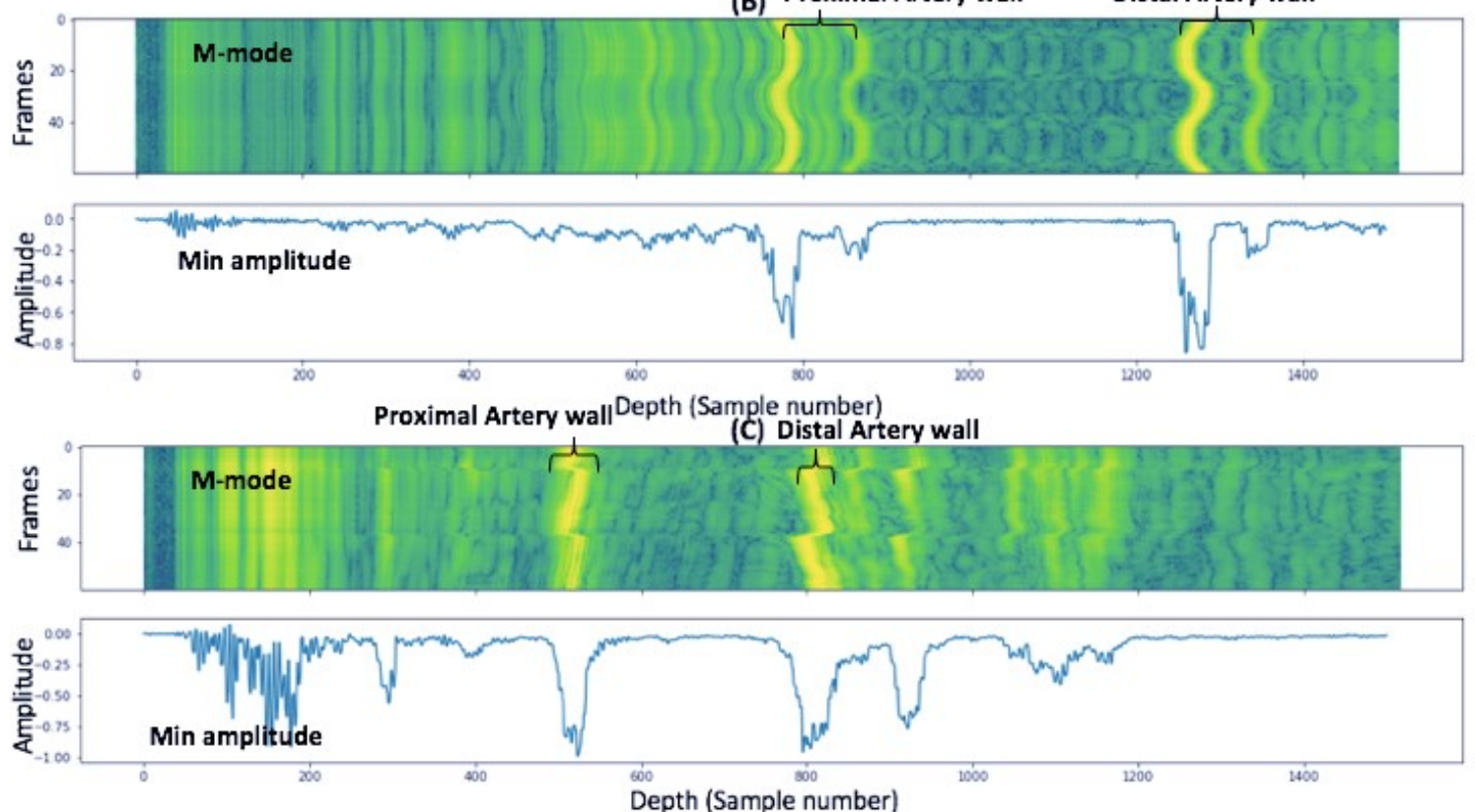

Figure 4.16 M-mode signal and the corresponding minimum amplitude along slow-time for (A) Phantom A, (B) Phantom B, (C)

In-vivo.

The distribution of the minimum amplitude averaged in each subsection along the slowtime is given in Figure 4.17-Figure 4.22. 
(A)

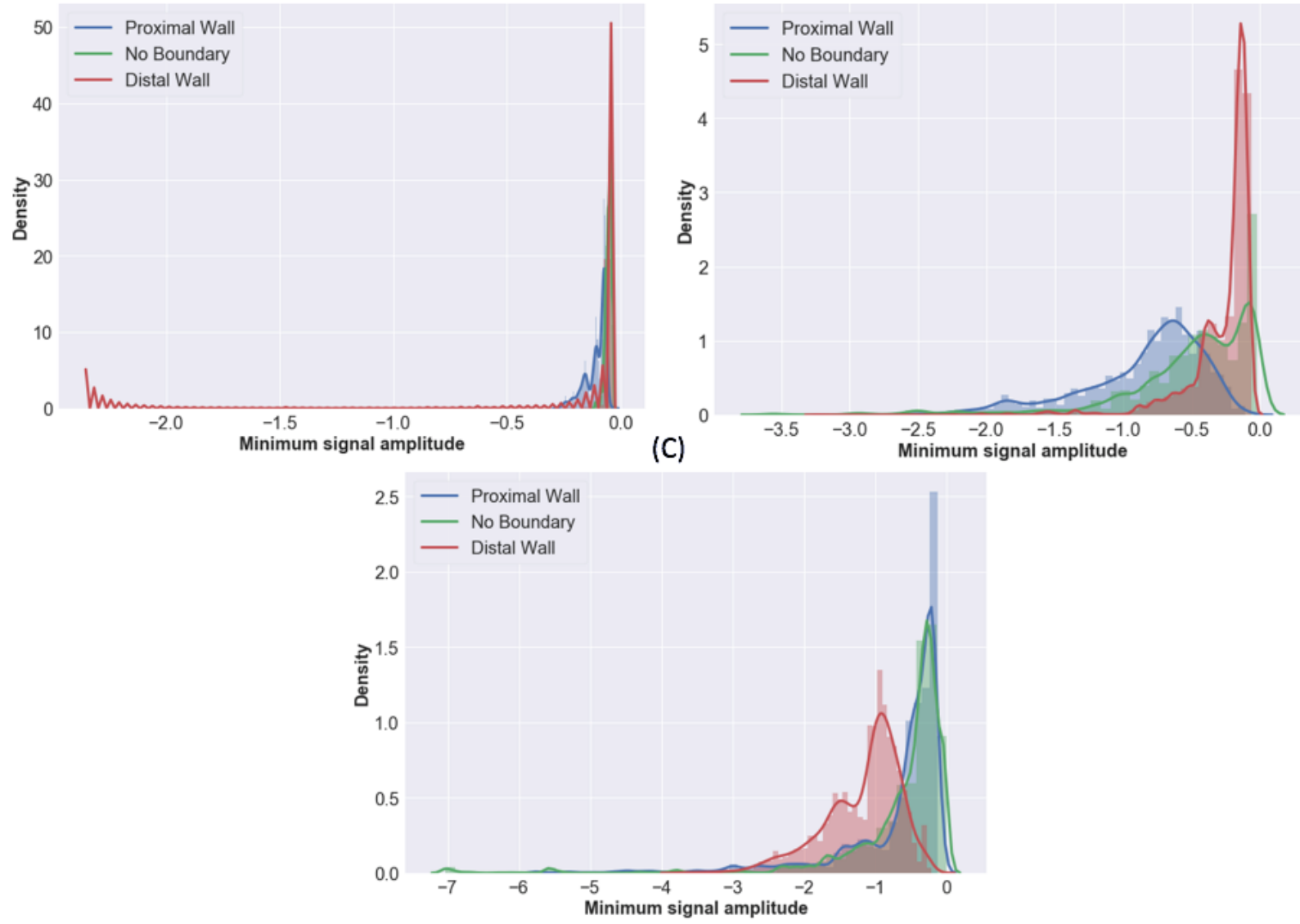

Figure 4.17 Distribution of minimum amplitude values of $D R_{1}$ values along slow-time for (A) Phantom A, (B) Phantom B, (C)

In-vivo. 
(A)
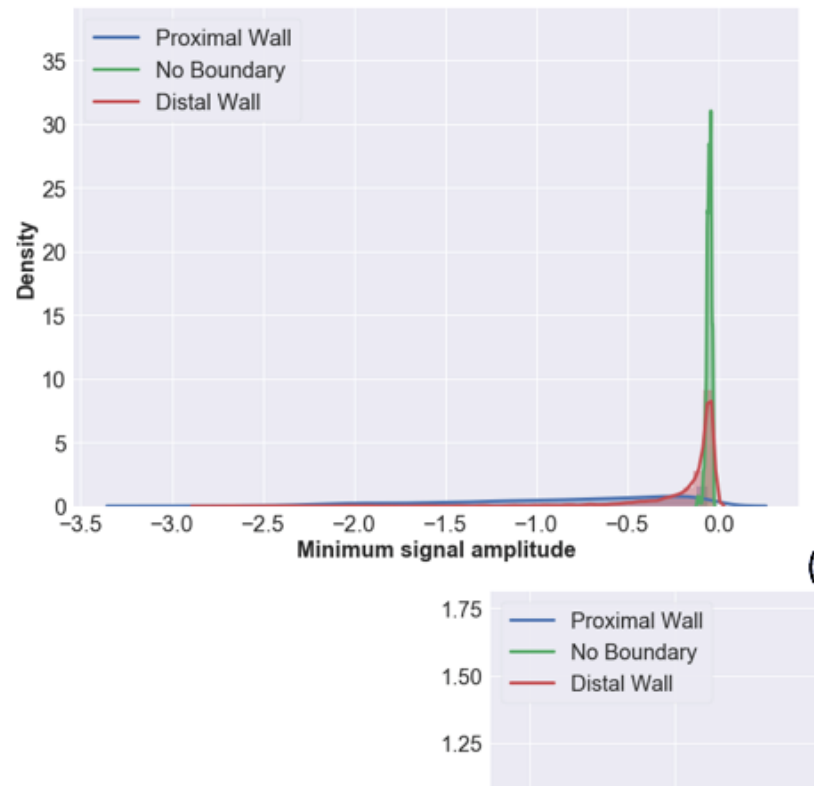

壳 1.00

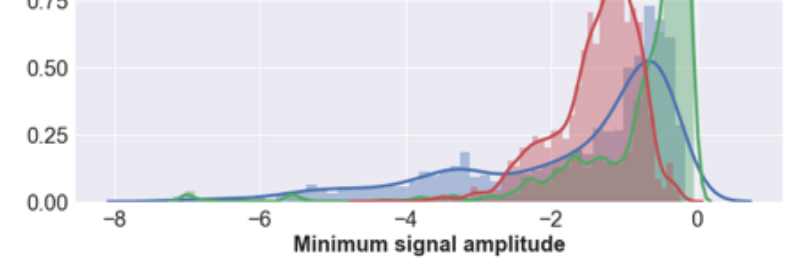

Figure 4.18 Distribution of minimum amplitude values of
(B)

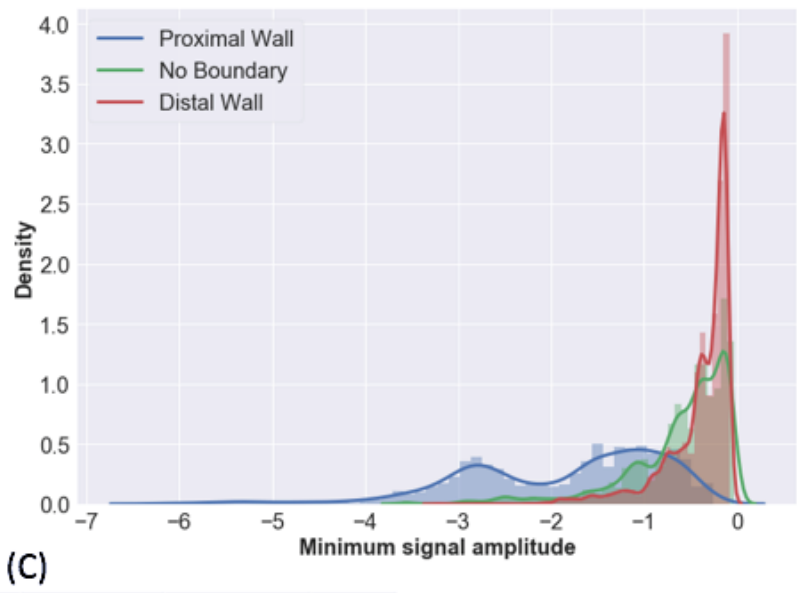

(C)

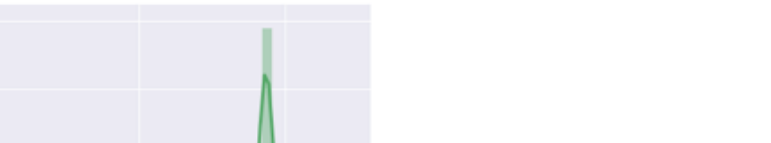


(A)

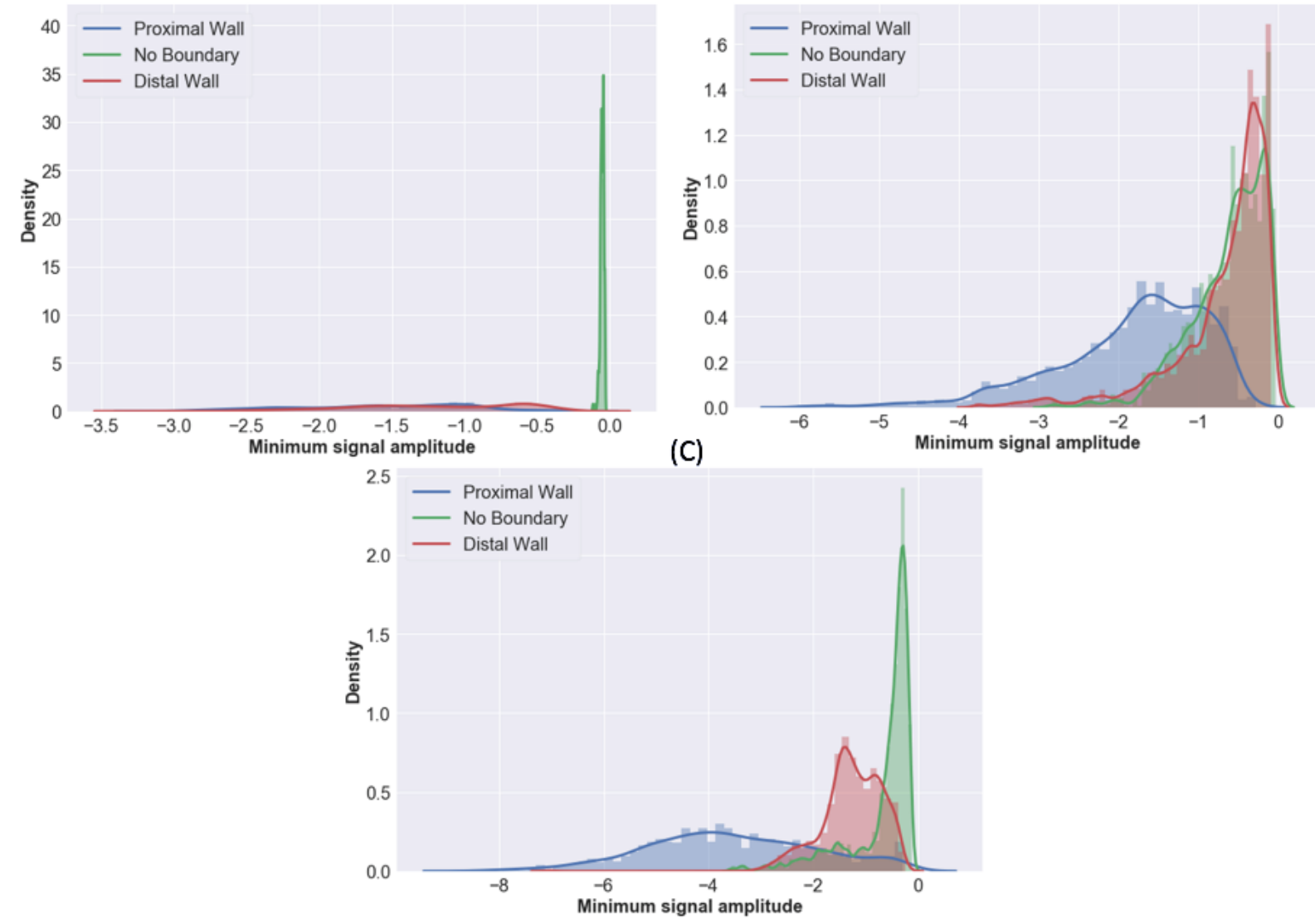

Figure 4.19 Distribution of minimum amplitude values of $D R_{3}$ along slow-time for (A) Phantom A, (B) Phantom B, (C) Invivo. 
(A)
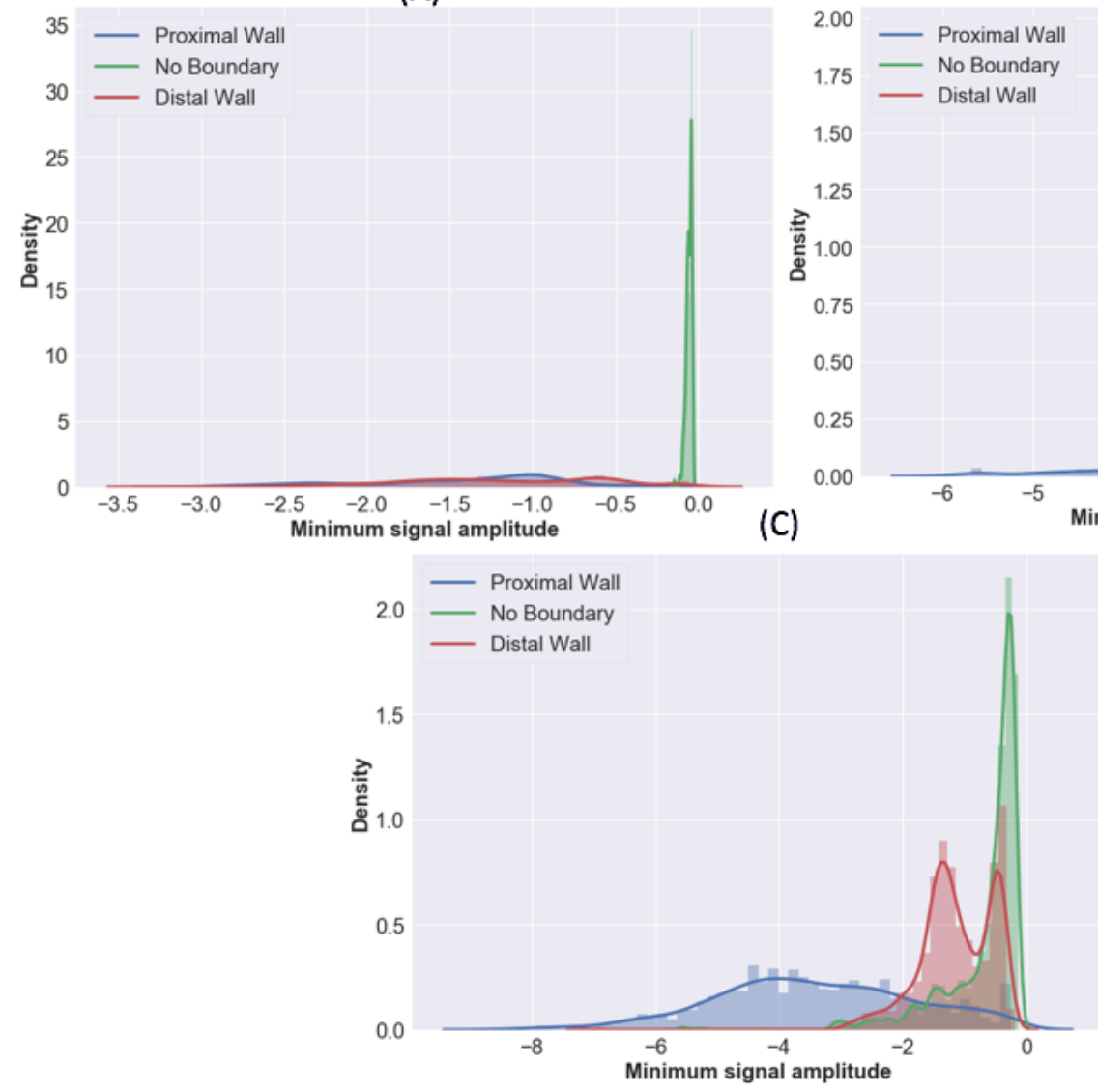

(B)

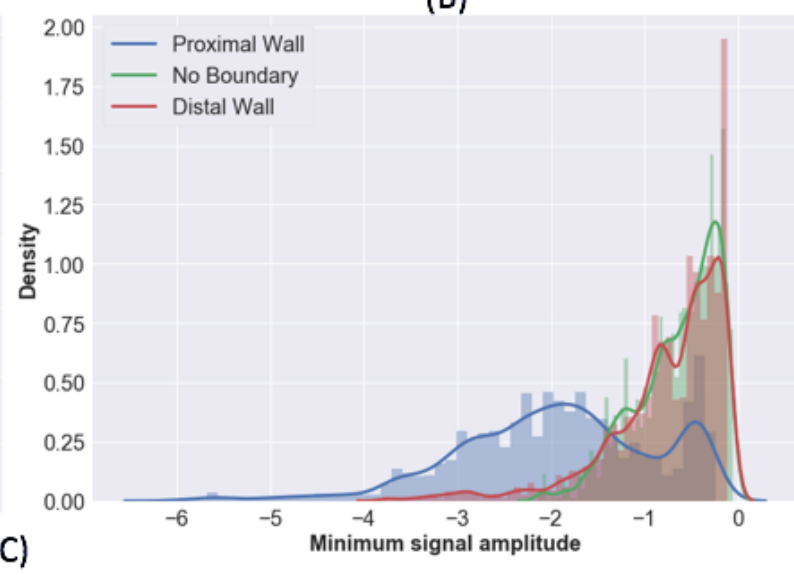

Figure 4.20 Distribution of minimum amplitude values of $D R_{4}$ along slow-time for (A) Phantom A, (B) Phantom B, (C) In-vivo. 
(A)
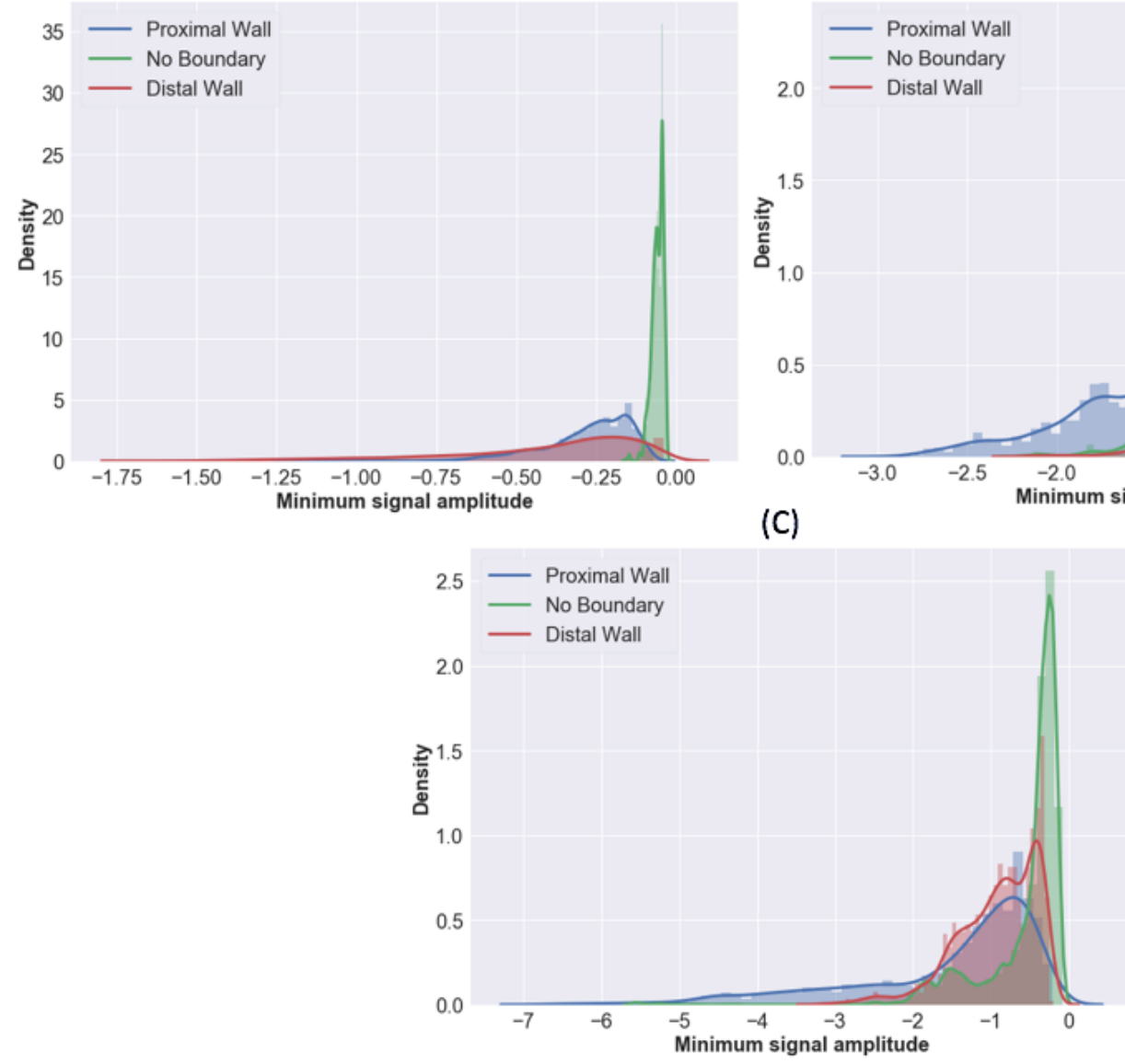

Figure 4.21 Distribution of minimum amplitude values of
(B)

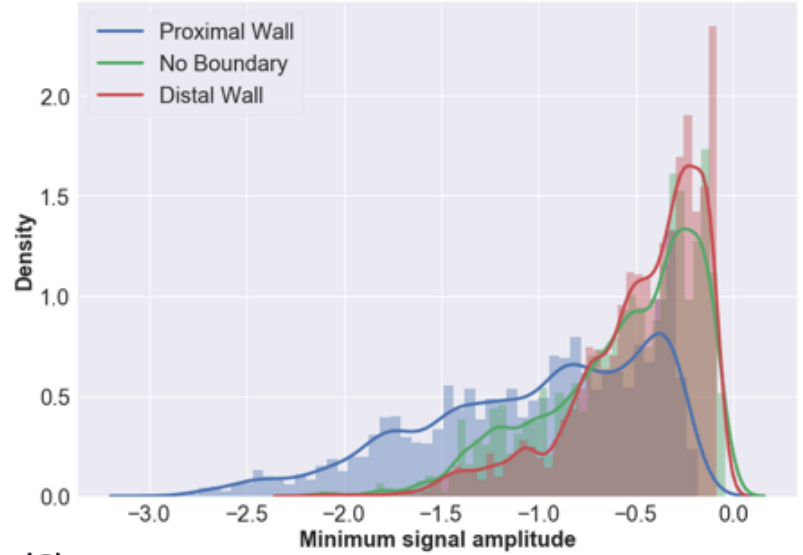

(C)

\section{$D R_{5}$ along slow-time for (A) Phantom A, (B) Phantom B, (C) In-}

vivo.

It is observed from the above Figure 4.17- Figure 4.21, that the distribution of the averaged values of minimum amplitude values along the slow-time of different segments is more separated in the case of Phantom A than the cases of Phantom B and in vivo subject. In the case of Phantom B and in-vivo based experiments, as observed in Figure 4.16, there is an overlap in the distribution of the averaged values due to the scattering echo noise from the stationary tissues. 


\section{Fractal dimension}

The concept of fractal dimension (FD) is a generalization of the Euclidean dimension that reflects the complexity of the self-similar structure of the object [84]. The rows of the segmented window are considered as a time-series as the same pattern of the artery wall motion is observed along the slow-time. This self-similarity of the time series translates to scale-invariant complexity. The FD is used in this thesis as the tissue characterizing feature as it provides a measure of "scale-invariant roughness" even when the object is not a fractal [84]. The FD is calculated for all the rows of the segmented window. Since, the rows of the segmented window are a time series, the FD of all the rows acts as a measure of the scale-invariant complexity of each row. The method to calculate the FD value is given in Appendix.

The FD is predominantly used in the case of analysis of biomedical signals such as EEG [85]. Research has been done on the time series formed by numerous biological processes including blood pressure and heart rate [86]. The primary assumption in most of these studies is that the fractal dimension of a time series reveals properties that are raised from complex internal nonlinear dynamics of the underlying process [84] (rather than those caused by environmental conditions and external noises). Based on this assumption, the FD is utilized in characterizing the tissue feature of the ultrasound $\mathrm{rf}$ signal in this thesis.

Figure 4.22 shows M-mode signal and the fractal dimension of the time series obtained across different depths. In [87], it is presented that the fractal dimension value of 2 is an indicator of a white noise time series, and any correlated randomness in the time series 
would alter the fractal dimension value from 2. A correlated randomness is defined as the correlation between the values that can be observed in a random signal. The artery wall moves in a quasi-periodic motion due to the cardiac cycle. However, due to the other factors such as scattering noise and motion artifact, the time series observed at the depth where the artery wall is present exhibits correlated randomness in Fig. 4.22. The time series of the ultrasound rf signals observed at a depth where there is no artery wall exhibits complete randomness as there is no periodic motion-induced unlink the case of the artery wall. Figure 4.23 shows the fractal dimension observed at different depth for ultrasound rf signals acquired with Phantom A, Phantom B and human subject by PICUS clinical imaging system in M-mode. 


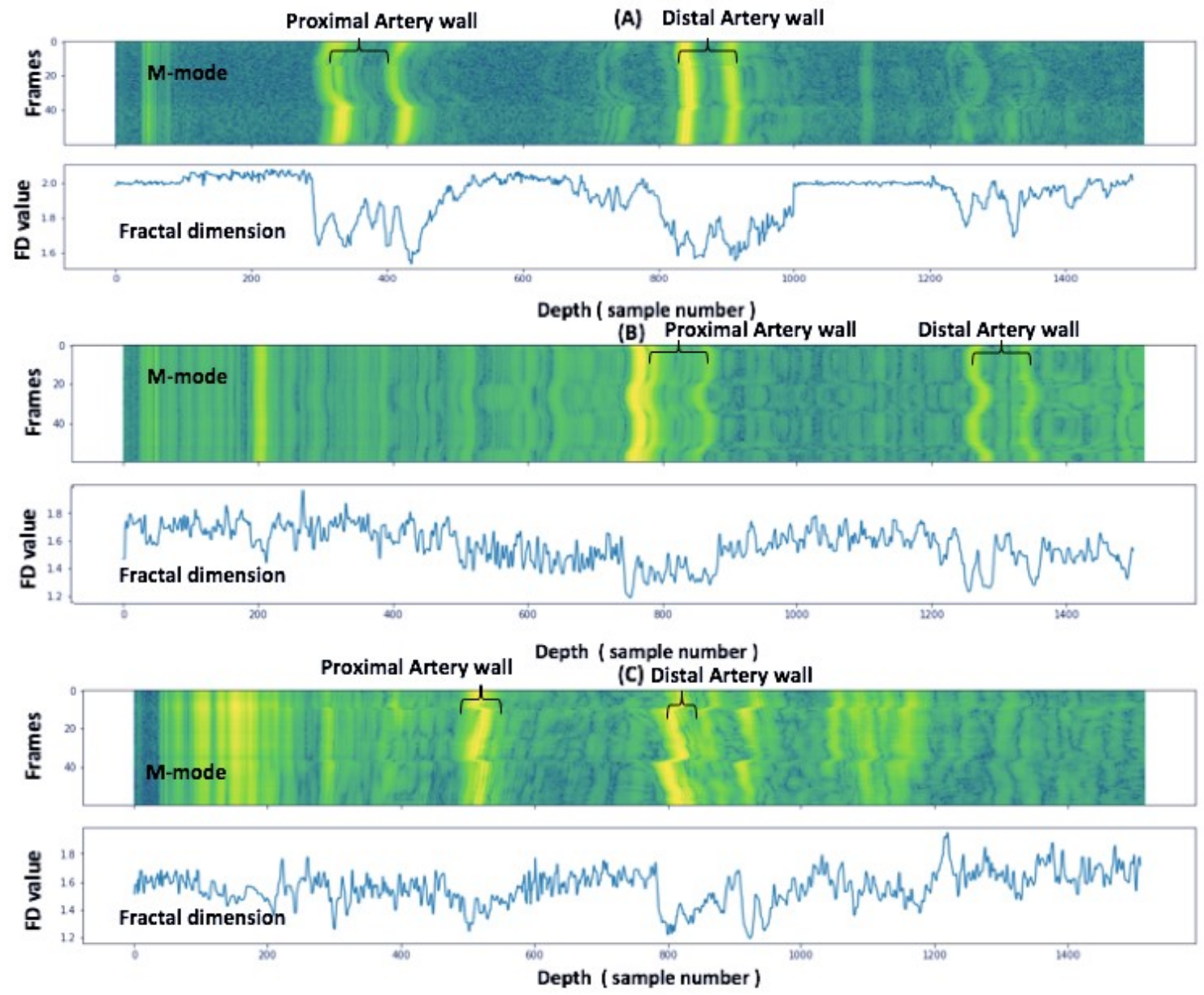

Figure 4.22 M-mode signal and the corresponding fractal dimension values along slow-time (A) Phantom A, (B) Phantom B, (C) In-vivo.

By observing Figure 4.22, it can be seen in the case of the water-based Phantom A that the value of the FD (approximately 2) of the time series in the region where there is no artery wall is completely different as compared to the region with the artery wall (approximately 1.6). But, in the case of the gelatin-based Phantom B and the in-vivo human subject, the value of the fractal dimension that is observed in the region where the artery walls are present is approximately in the range of 1.2-14, which is not much different from the 
region surrounding the artery walls, which approximately ranges from 1.4-2.0 . This is probably due to the movement of the surrounding tissues along with the artery walls. The FD value observed for a time series with a correlated randomness is lower (around 1.2), while the FD of the time series of a random signal is around 2. The time series observed at the depth, the artery walls are present exhibits correlated randomness due to the quasiperiodic motion of the artery walls. The quasi-periodic movement of the artery wall due to the cardiac cycle is also propagated to the tissues that are immediately surrounding the artery walls. Due to this, the time series (rows representing the time series of the surrounding tissues of the artery) observed in the region of the surrounding tissues of the artery wall also exhibit correlated randomness. The time series representing the stationary tissues has FD value that is different from the time series representing the moving tissue. The distribution of the average fractal dimension value for each of 5 subsections of the segmented window is given in Figure 4.23- Figure 4.27. 
(A)
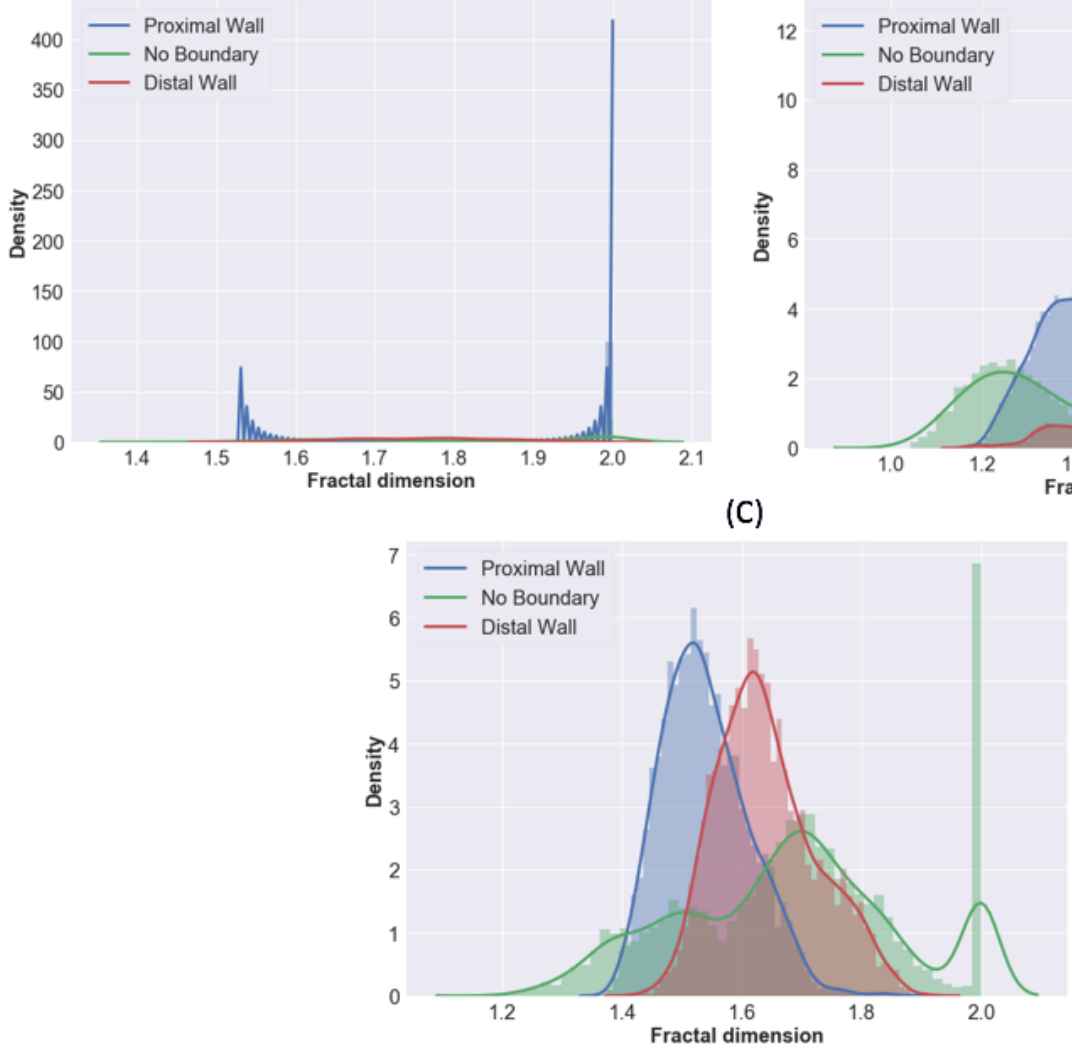

(B)

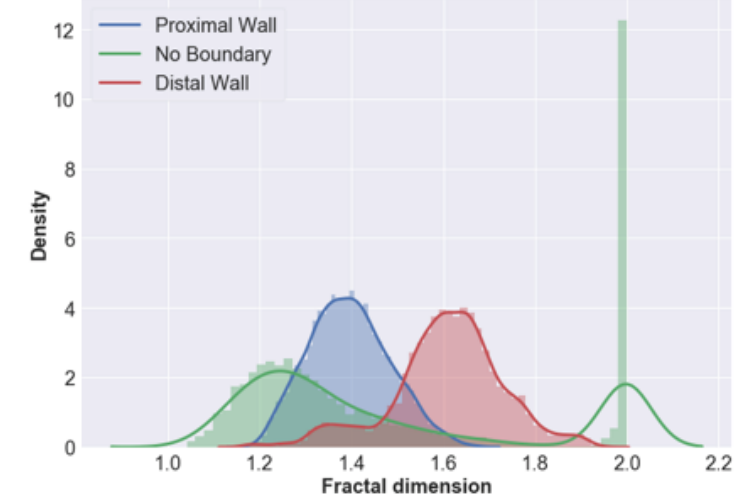

(C)

Figure 4.23 Distribution of fractal dimension value of $D R_{1}$ along slow-time for (A) Phantom A, (B) Phantom B, (C) In-vivo. 
(A)

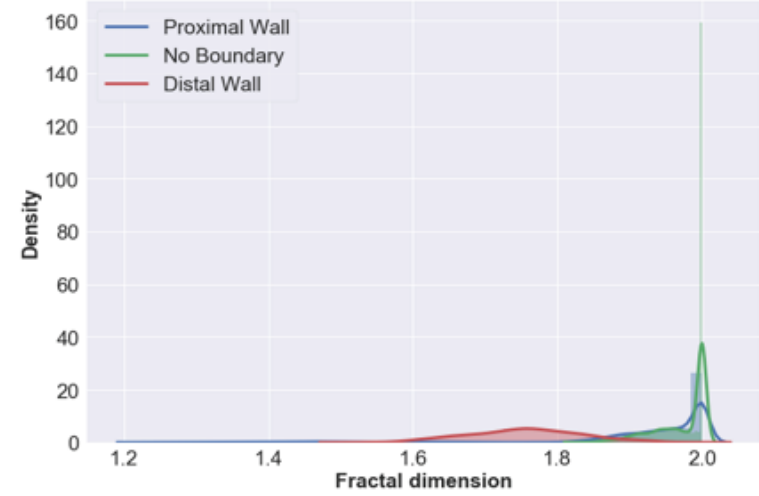

(B)

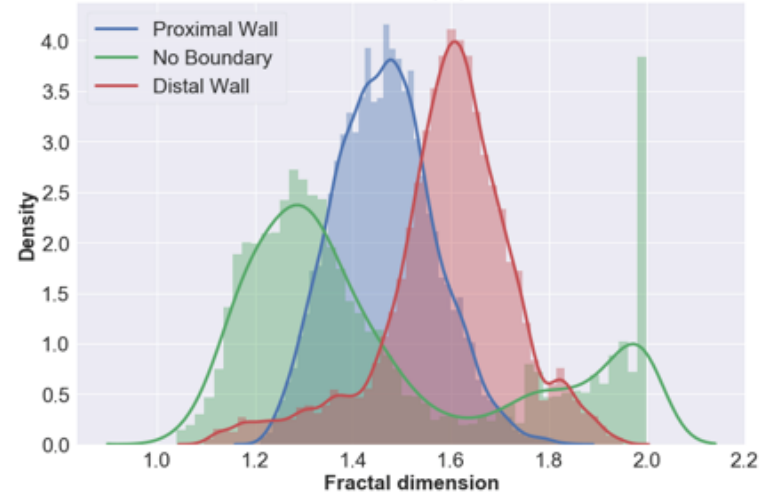

(C)

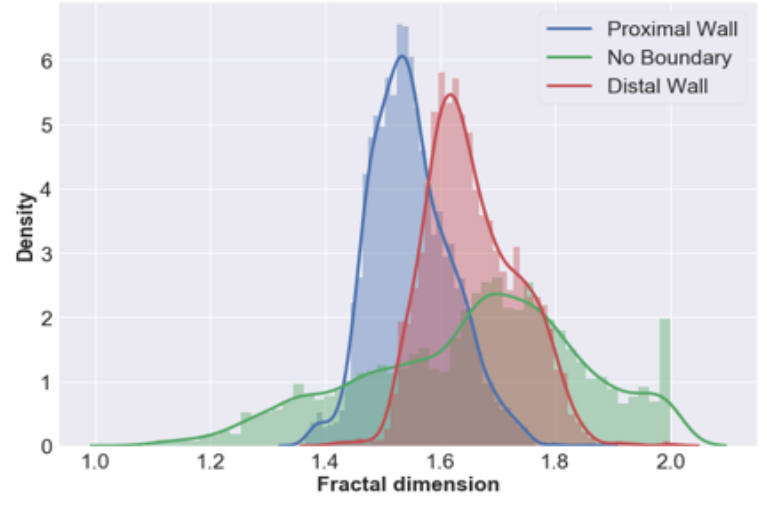

Figure 4.24 Distribution of fractal dimension value of $D R_{2}$ along slow-time for (A) Phantom A, (B) Phantom B, (C) In-vivo. 
(A)

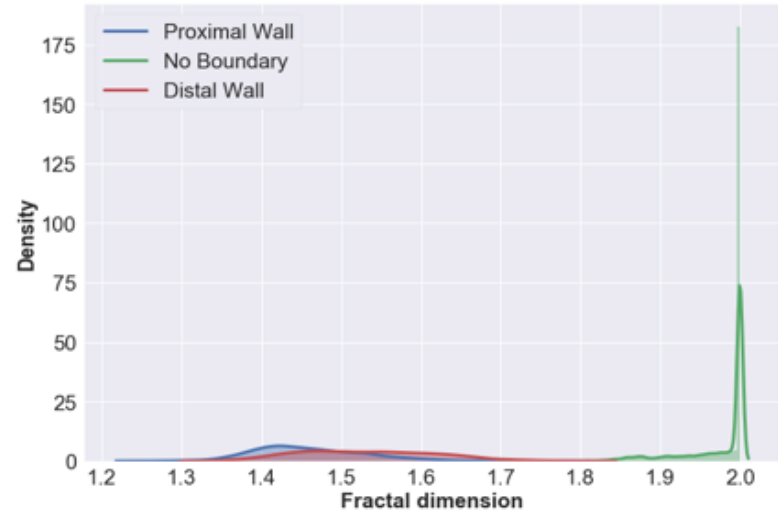

(B)

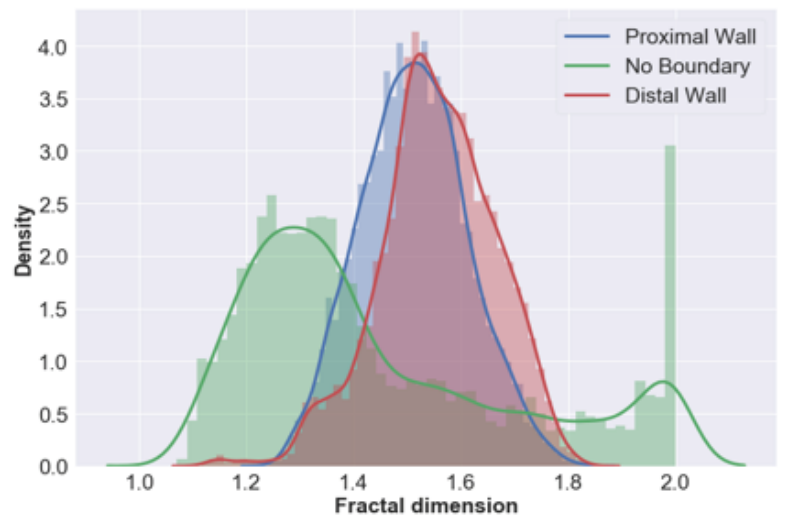

(C)

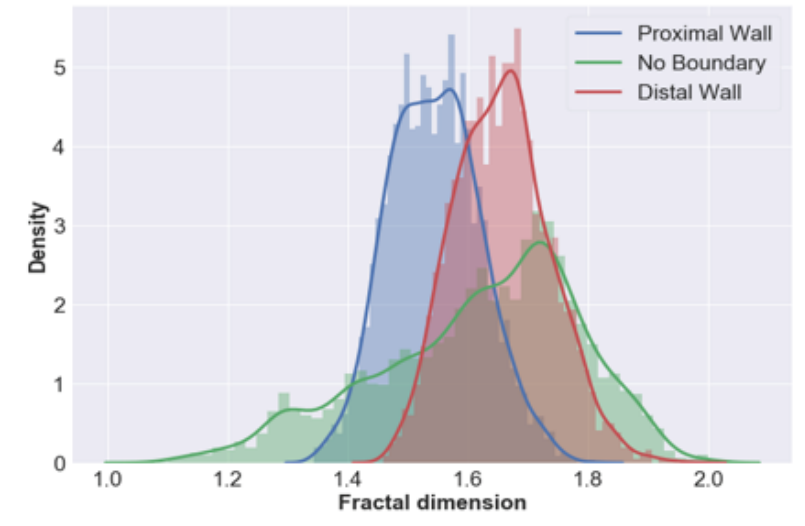

Figure 4.25 Distribution of fractal dimension value of $D R_{3}$ along slow-time for (A) Phantom A, (B) Phantom B, (C) In-vivo. 
(A)

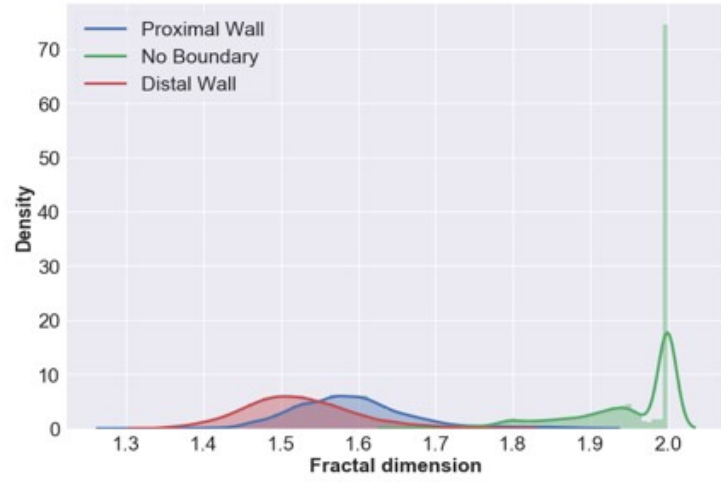

(B)

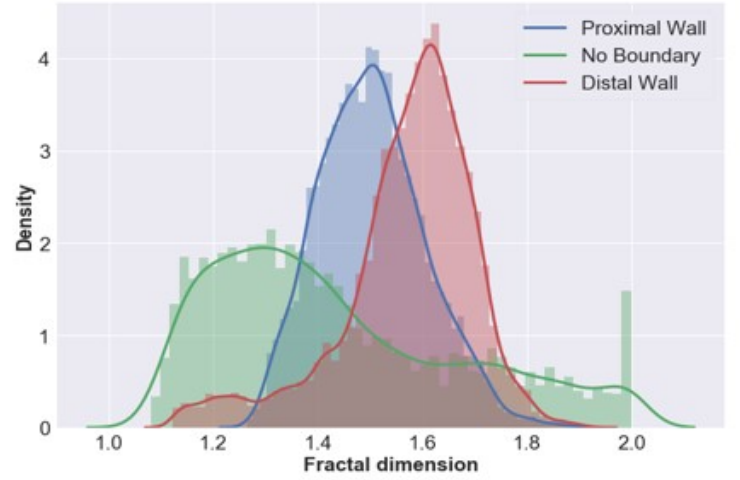

(C)

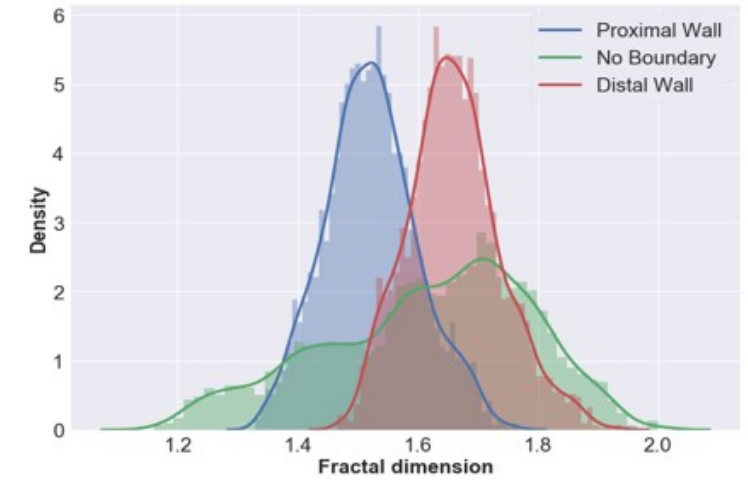

Figure 4.26 Distribution of fractal dimension value of $D R_{4}$ values along slow-time for (A) Phantom A, (B) Phantom B, (C) In-vivo. 
(A)

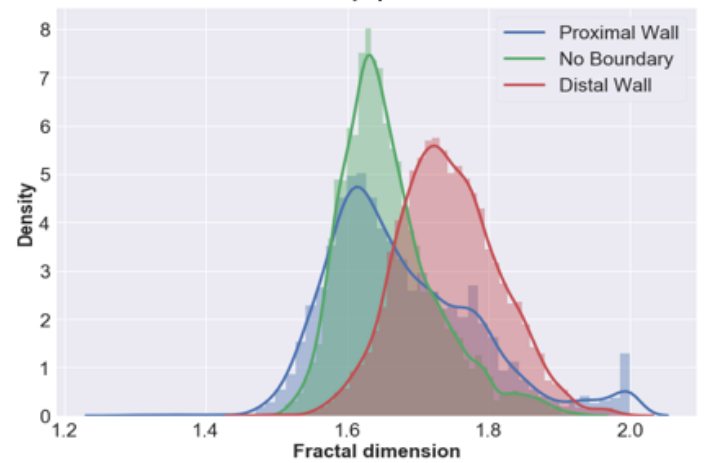

(B)

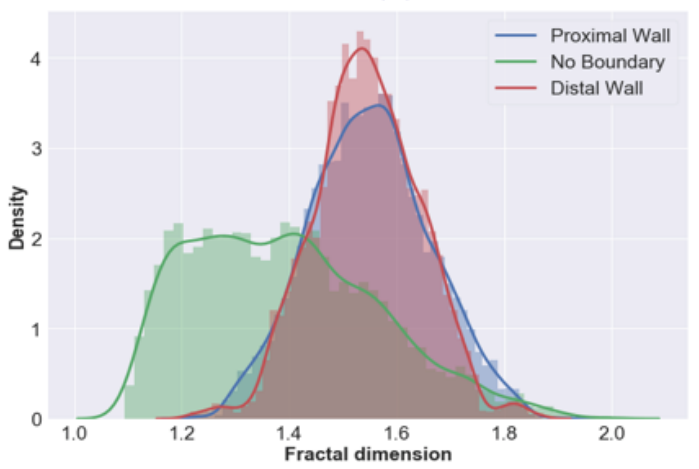

(C)

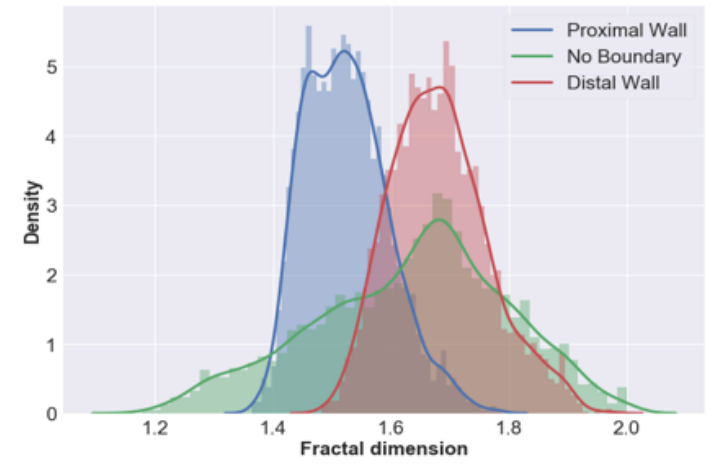

Figure 4.27 Distribution of fractal dimension value of $D R_{5}$ along slow-time for (A) Phantom A, (B) Phantom B, (C) In-vivo.

On observing the distribution of the FD values for the segmented window in Figures 4.234.27 , it can be seen that the average of the FD values in the case of the water-based phantom A clearly distinguishes the region containing the artery wall and the region without it, despite the bounce-back (as explained in Section 3.2.1.2) that is observed in Phantom A. In the case of the Phantom B and the in-vivo experiments, there is an overlap in the distribution of the average values of the fractal dimensions. The overlap in the distribution of the FD may be attributed to the fact that the region that does not contain the artery wall but close to the artery wall also moves due to the motion of the artery walls to an extent. This induced motion of the surrounding tissues causes the similar pattern to be produced 
in the time series as that of the artery walls, which in turn has similar FD values as that of the time series pattern created by the moving artery walls.

From Figure 4.22-4.27, it is seen that the fractal dimension along the slow time domain of the artery wall is a weak feature, as it is not able to clearly distinguish the artery wall from the no boundary region. But the value of fractal dimension, in combination with the other slow and fast-time features would be beneficial for the machine learning classifiers to classify the segmented regions in higher dimensions. Similarly, the manually extracted features such as the energy, max and min when used in conjunction with the features predicting the periodicity can be used to create a strong feature. The combination of the features when projected on a higher dimension could bring up the distinguishable pattern that can be classified by the machine learning classifiers.

\subsubsection{Feature pre-processing}

The extraction of features for a given segmented window is explained in Sections 4.1.2 and 4.1.3. The features obtained for a given segmented window are of different metrics and scale. Hence a feature pre-processing technique is used to standardize the feature values. The effect of scaling the features in the performance of the machine learning algorithm is discussed in [84],[88]. For a given feature X, the standard score of $z$ is calculated using the Equation (4.11).

$$
z=\frac{\left(X-X_{\text {mean }}\right)}{\sigma_{X}}
$$

Where $\mathrm{X}$ is the value of the feature considered, and $\sigma_{X}$ is the standard deviation of the feature extracted $X$. The scaling process redistributes the features with their mean value of 
$\mathrm{z}, z_{\text {mean }}=0$ and standard deviation of $\mathrm{z}, \sigma_{z}=1$. It helps all features to be on the same scale relative to one another. Since the number of segmented windows representing the proximal and distal wall is less as compared to the number of segments with no boundaries, and there is a class imbalance in the dataset obtained. Training the classifier with the class imbalance will introduce a bias in the classifier. In order to address this problem, synthetic minority oversampling technique (SMOTE) is used to create a balanced dataset to train the classifier [89].

\subsubsection{Machine learning algorithms}

In this section, the performance of different machine learning (ML) algorithms for a given set of features presented in Sections 4.1.2 and 4.1.3 are compared against each other. Since there is no benchmark established to identify the effectiveness of the features considered, a group of machine learning algorithms are chosen and compared against each other. The performance of the model depends on the features selected. Chosen ML algorithms considered for comparison in this thesis are Linear Discriminant Analysis (LDA), KNearest Neighbor (KNN), Random Forest (rf), and Extreme Gradient Boosting (XGB). In addition to the machine learning algorithms mentioned above, the performance of Convolutional Neural Network (CNN) which extracts the features automatically from the segmented window, is also compared with the rest of the algorithms. The algorithms to be discusses in this thesis are chosen in such a way that it includes a parametric model (LDA), non-parametric model (KNN), meta-learning tree-based models (RF and $\mathrm{XGB}$ ), and a 
neural network-based model $(\mathrm{CNN})$. The metrics used to compare the performance of the chosen algorithms are: Accuracy, F1 score, Area under the ROC curve, and the PrecisionRecall curve. Choosing the most suitable ML technique comes with a trade-off between the advantages and disadvantages that each approach possesses. The following algorithm is trained on the features discussed in Sections 4.1.2 and 4.1.3 to classify the segmented window into one of the three classes of a proximal artery wall, distal artery wall, or no artery wall boundary regions.

\subsubsection{Linear Discriminant Analysis (LDA)}

The Linear discriminant analysis (LDA) is used, as it is a simple and powerful linear classification algorithm that addresses the limitation of the logistic regression for the case of multiclass unbalanced dataset [30]. As explained in Section 2.1.2.1, LDA attempts to find linear combinations of predictor variables that best separate the groups of observations. The underlying assumption of LDA is that the features considered are Gaussian distribution and each feature has the same variance. The LDA projects the features that are derived in the higher dimension onto the lower-dimensional space. At first, the separability between the different classes is obtained by calculating the distance between the mean of the three classes (proximal artery wall, distal artery wall, and no artery wall boundary), which is called the between-class variance. Secondly, the distance between the mean and the sample of each class called the within-class variance is calculated. Since the actual mean vectors and covariance matrices are almost always unknown, the maximum likelihood estimates are used to estimate these parameters. The eigenvalues and the 
eigenvector of the scatter matrices (between-class and within-class variance scatter matrix) are calculated. The first $\mathrm{k}$ sorted eigenvectors by the decreasing order of their eigenvalues are selected, and a lower-dimensional space is constructed. Lastly, a boundary is built to separate the classes by maximizing the between-class variance and minimizing the withinclass variance. In this thesis, linear discriminant is implemented with the help of the Scikit learn library [42].

\subsubsection{K- nearest neighbor (KNN)}

K-nearest neighbor is an instance-based learning explained in Section 2.1.2.2. Unlike the LDA, this algorithm does not assume the distribution of the features. The input features that are obtained in Sections 4.1.2 and 4.1.3 are represented as points in an n-dimensional space where $\mathrm{n}$ is the number of the features. Classification is computed from a simple majority vote of the nearest neighbors of each point: a query point is assigned to the data class, which has the most representatives within the K-nearest neighbors of the data point. The value of $\mathrm{K}$ is highly data-dependent and is selected with the process of grid searching for the different experimental cases [42], [90]. In order to assign higher priority to the classes with a lesser number of samples, the points representing the classes with the lower number of training samples can be assigned with higher weight values. However, the number of training samples is ensured to be the same; the weights to the neighbors are assigned to be uniform. The distance between the points is calculated using the Minkowski distance formula since it is a metric in the normed vector space [91]. The value of $K$ is selected experimentally for each experimental condition with the help of the grid-search algorithm given in [42] The main disadvantage of the K- nearest neighbor algorithm is that the computational complexity of the test stage of the classifier is expensive [33]. 


\subsubsection{Random Forest (RF)}

The decision tree is a non-parametric supervised learning method that creates a model that predicts the value of the target variable by learning simple decision rules inferred from the data features. A random forest is a meta estimator that fits several decision tree classifiers on various sub-samples of the dataset and uses averaging to improve the predictive accuracy and control over-fitting. The sub-samples of the dataset are of the same size as the original data set acquired by bootstrapping the original training data. The algorithm is implemented with the help of the library [92]. The hyperparameter of the maximum depth is selected with the help of the grid search technique presented in [42]. Since the hyperparameter of minimum samples per split is dependent on the depth of the trees and adjusted accordingly, it is set to a default value of 2. Each of the features is given equal importance at the beginning, and no weight is provided for any feature. Random Forest, as mentioned in Section 2.1.2.3, has the main advantage of RF is its robustness to outliers and missing values in training data.

In contrast to that, the main disadvantage of random forest is that it is constructed of multiple fully grown decision trees with unreliability. Unreliability in this context means that the splitting attribute in a high-level node can change by only adding a few additional data entries, changing the structure of the whole tree, and the corresponding predictions. Further, the random forest is constructed using multiple fully grown trees; the training time of the algorithm is high [33]. 


\subsubsection{Extreme gradient boosting (XGB)}

Extreme gradient boosting is a parallel implementation of the gradient boosted decision trees as explained in Section 2.1.2.4. This ensemble learning technique rectifies the problem of the higher computational time of the random forest. Unlike the random forest, where the trees are fully grown, the extreme gradient boosting trees are made up of multiple undergrown trees, which are weak learners. The hyperparameters that are considered for the extreme gradient boosting are learning rate for the gradient boosting, maximum depth of the trees, subsample which is the percentage of samples used per tree, co-sample by tree which is the percentage of features used per tree, number of estimators: the number of trees that is to be build. Since the boosting technique is used to address the error that is induced because of the variance and not the error due to the bias [39]. The parameters specified above are selected experimentally with the help of the grid search technique [42] for all the experimental conditions explained in Section 3.2. The extreme gradient boosting algorithm is implemented with the help of the sci-kit learn library [92].

\subsubsection{Convolutional neural network (CNN)}

The convolutional neural network [44] is used to classify the subsets of the M-mode image (i.e., segmented windows) into either of the three classes. The architecture of the convolutional neural network used for the case of the in-vivo experiment is given in Table 4.1. 
Table 4.1 Architecture of CNN.

\begin{tabular}{|c|c|}
\hline Layer Type & Output shape \\
\hline Convolutional layer 1 & $(200,60,32)$ \\
\hline Max pooling layer 1 & $(100,30,32)$ \\
\hline Convolutional layer 2 & $(100,30,16)$ \\
\hline Max pooling layer 2 & $(100,15,16)$ \\
\hline Convolutional layer 3 & $(50,15,16)$ \\
\hline Max pooling layer 3 & $(50,15,16)$ \\
\hline Dropout layer 1 & $(50,15,16)$ \\
\hline Convolutional layer 4 & $(50,15,8)$ \\
\hline Max pooling layer 4 & $(25,7,8)$ \\
\hline Dropout layer 2 & $(25,7,8)$ \\
\hline Flatten & 1400 \\
\hline Dense layer & 3 \\
\hline
\end{tabular}

The size of the input layer is selected according to the size of the segmented window, while the number of output classes dictates the output layer's size. The number of hidden dense, convolution and pooling layers is selected experimentally by taking into consideration of the number of trainable parameters and the size of the training data set. The convolutional layers have rectified linear units as the activation function, while the output dense layer has SoftMax activation function [44] as given in the Equations 4.12 and 4.13, to convert the obtained probability into one of the three classes [44]. 


$$
\begin{gathered}
\operatorname{softmax}(x)_{i}=\frac{\exp \left(x_{i}\right)}{\left.\sum_{j} \exp \left(x_{j}\right)\right)} \\
\operatorname{Relu}(x)=\max (x, 0)
\end{gathered}
$$

In the case of the convolutional neural network, the network itself extracts the feature from the segmented window [93]. Since the movement of the artery walls in the case of the proximal and the distal walls are opposite to each other, the classifier learns to differentiate between the segments containing two different sections of the artery walls. The segmented window images observed in different experimental conditions are given in Figure 4.28 Figure 4.31.

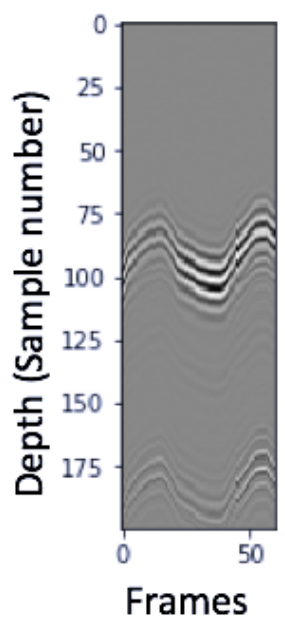

(A)

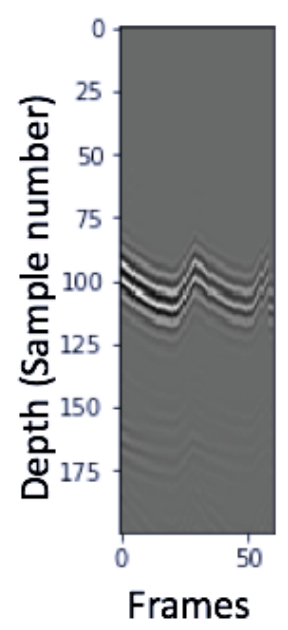

(B)

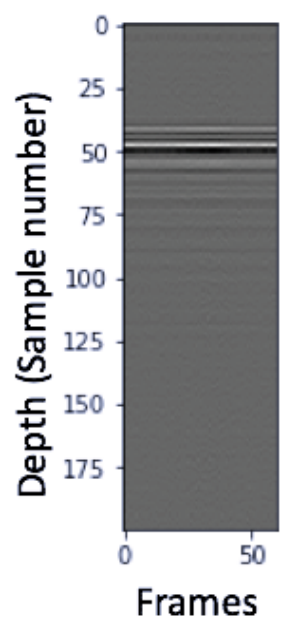

(C)

Figure 4.28 Segmented windows containing (A) proximal wall, (B) distal wall, (C) no boundary for Phantom A obtained with PICUS clinical ultrasound imaging system. 


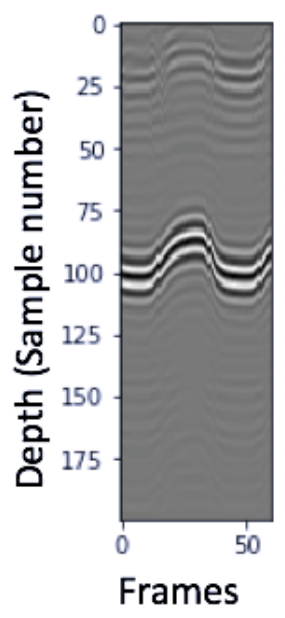

(A)

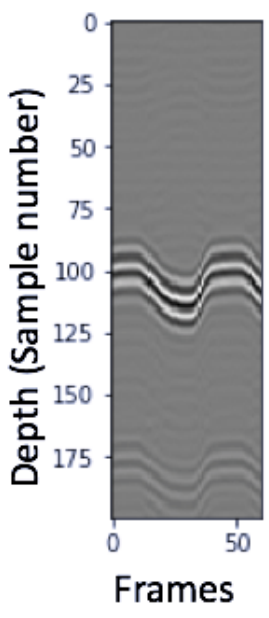

(B)

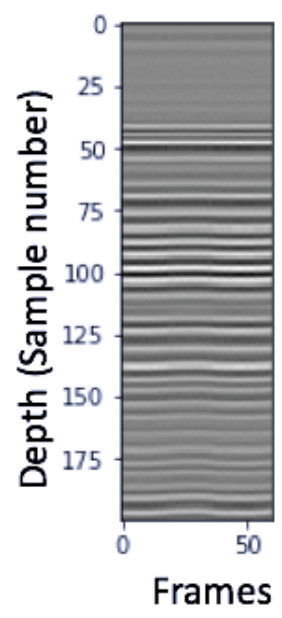

(C)

Figure 4.29 Segmented window containing (A) proximal wall, (B) distal wall, (C) no boundary for Phantom B obtained with PICUS clinical ultrasound imaging system. 


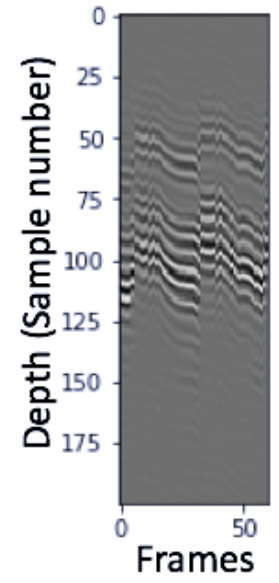

(A)

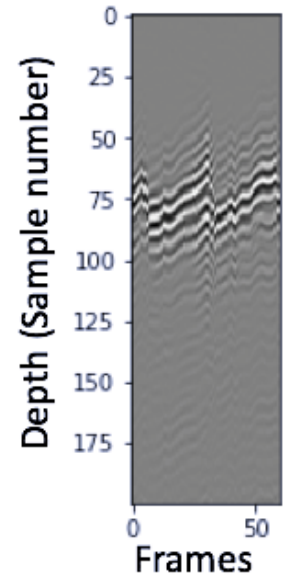

(B)

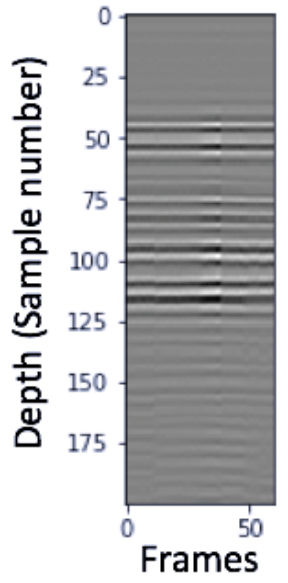

(C)

Figure 4.30 Segmented window containing (A) proximal wall, (B) distal wall, (C) no boundary for in-vivo human subject obtained with PICUS clinical ultrasound imaging system. 


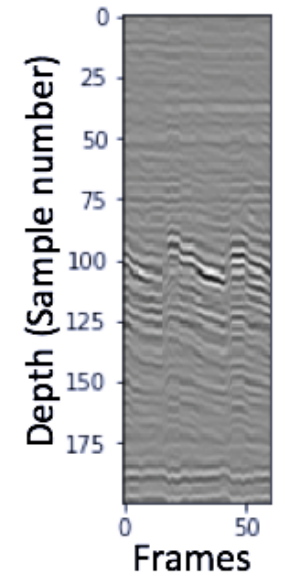

(A)

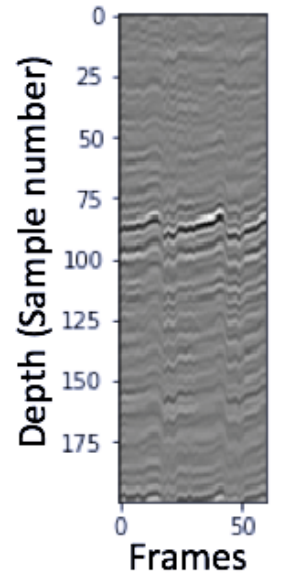

(B)

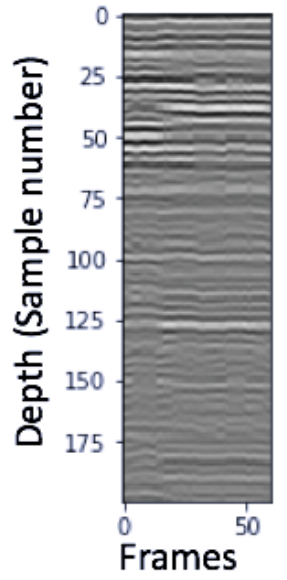

(C)

Figure 4.31 Segmented window containing (A) proximal wall, (B)

\section{distal wall, (C) no boundary for human in-vivo subject obtained with Wearable Ultrasound Sensor (WUS) system.}

It is observed from Figure $4.28-4.31$ that the rf signal pattern that is induced in the proximal and distal artery wall due to the heartbeat is quasi-periodic and opposite to each other. Thus, when the convolutional neural network is given with the rf signal data of the proximal wall, distal wall, and no boundary, the neural network learns the pattern that is observed in the respective walls. The loss function of categorical cross-entropy (CE) given by the Equation (4.14), is used to train the model. Stochastic gradient descent is used as an optimizer to reduce the loss function and to train the classifier [45].

$$
C E=-\sum_{i}^{C} y_{i} \log \left(\operatorname{softmax}(x)_{i}\right)
$$

Where $x_{i}$ is a resultant vector of the inputs, and the adjusted weights and $C$ is the total number of classes. Once the features are extracted for labeled segmented windows, as explained in Section 4.1.3, they are split into two data sets of training and validation data, 
and test data. Among the total labeled data, $80 \%$ of the total data is used for training and validation, while the remaining $20 \%$ is used for testing purposes. It is to be noted that all the classifiers are trained and tested on the data obtained from each of the experimental conditions explained in Section 3.2. (i.e., the classifier is trained and test on the data collected from the same experiment). After comparing the performance of the classifier to detect the artery wall region (to be discussed in Section 5.1), the classifier that performs best is selected to identify the artery wall region. The segmented windows of the M-mode image from a test data is given as an input to detect the artery wall regions. Once the classifier detects both proximal and distal walls, the range of depth of the segmented windows containing the proximal and distal wall is noted. To estimate the artery lumen diameter during systolic and diastolic pressure, the segmented windows classified as proximal and distal wall along with their depth (starting point) is noted and fed as an input to the artery lumen diameter estimation algorithm.

\subsection{Artery lumen diameter estimation}

In this section, the inner artery walls are identified using the peak detection technique and the lumen diameter of the artery is estimated in Section 4.2.1. The correlation-based artery wall tracking mechanism is explained in Section 4.2.2 and the results from the Section 4.2.1 and 4.2.2 is combined to estimate the change in the artery lumen diameter during the cardiac cycle. 


\subsubsection{Arterial inner wall detection}

Once the segmented window containing the proximal and the distal artery walls is found, it is necessary to locate the inner wall location (depth) of each artery wall, in order to obtain the lumen diameter. Since each column of the segmented window represents the A-mode rf signals at a particular region of depth, they are used to locate the lumen diameter at a specific instance of time. The distance between the two echo peaks of each wall, as shown in Figure 4.2, gives the thickness of each artery wall.

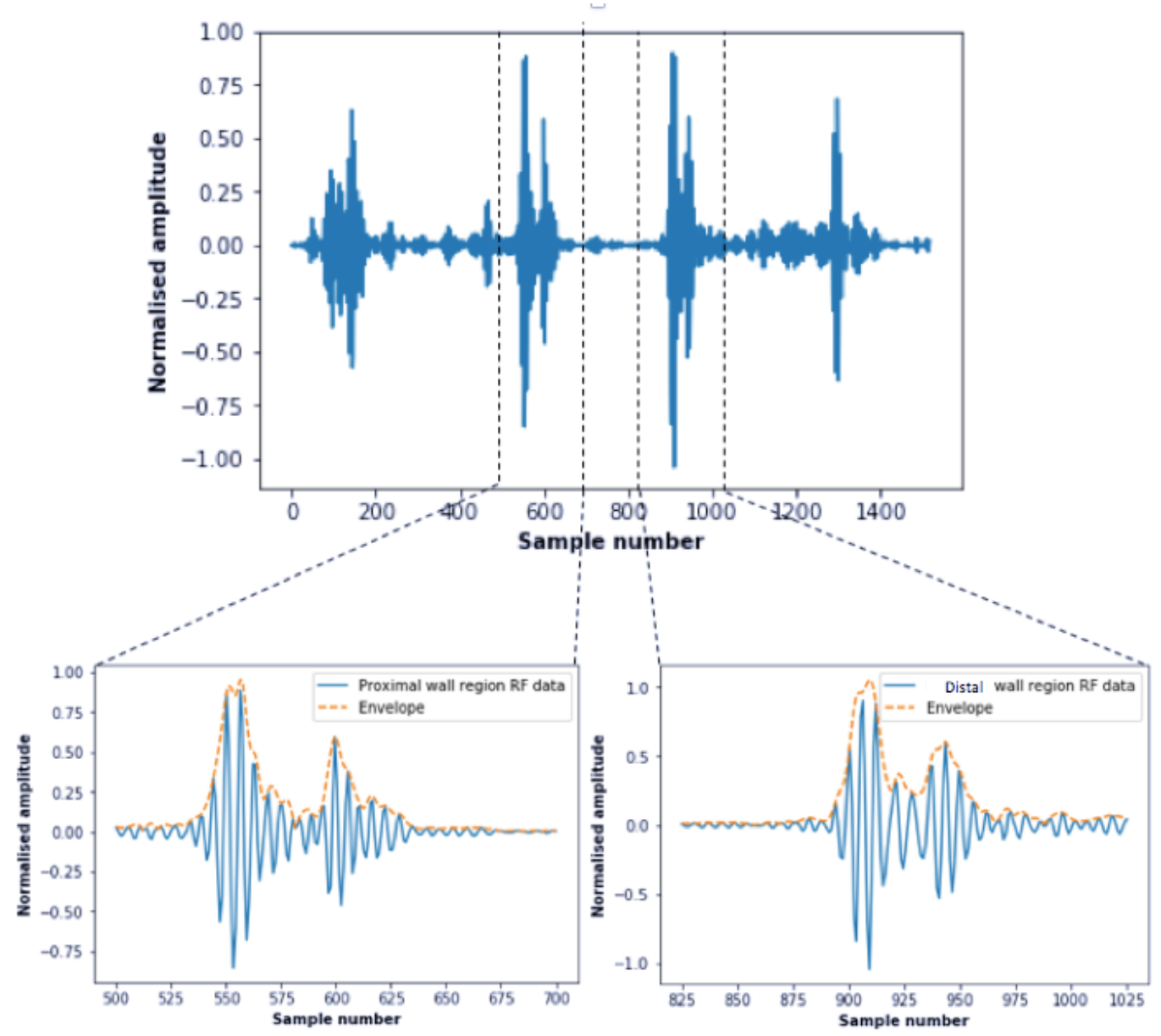

Figure 4.32 Segmented proximal and distal wall regions. 
It is observed from Figure 4.32 that in an rf signal from the segmented window containing either of the proximal wall or distal artery wall, there are two peaks which are representing the inner and outer walls, respectively. Since lumen diameter is defined by the distance between the proximal inner wall and distal inner wall, a peak detection technique on the rf signal envelop obtained by the Hilbert transform of the rf signal using Equations 4.1 and 4.2 is used to locate the peaks.

Let $P W=\{P(1), P(2), \ldots P(N)\}$, be the position of echo peaks located in the segmented window labeled as proximal wall using the peak detection technique [82]. $P(N)$ is selected to be the proximal inner wall echo $\left(\mathrm{P}_{\text {inner }}\right)$, because from Figure 4.2 it is observed that the inner wall echo occurs at the end of the segmented window containing proximal wall. Similarly, on considering $D W=\{D(1), D(2), D(3), \ldots . D(N)\}$ to be the position of the peaks located in the segmented window labeled as a distal wall, the location of the distal inner wall $D_{\text {inner }}$ is selected as $D(1)$ because the distal inner wall echo is the first echo peak observed in the segmented window labelled as distal wall.

The lumen diameter for the artery wall can be calculated as given in Equation 4.15

$$
L_{d}=t_{d} \times c / 2,
$$

where $t_{d}$ is the fast-time interval (along depth) between the $D_{\text {inner }}$ and $P_{\text {inner }}$. On knowing the location of the inner artery wall at each frame, the change in the diameter of the artery wall can be estimated. Thus, to track the location of the proximal and the distal wall along 
the frames, the technique of autocorrelation is used to find the distension of the proximal and distal artery walls.

\subsubsection{Autocorrelation-based tracking method of lumen diameter change}

The region in which the artery walls are present is identified with the help of the classifier, while the location of the artery inner walls is identified with the help of the peak detection technique. To identify the distension of the artery walls, the proximal and the distal wall region that is identified are correlated along the successive frames. Since the height of the segmented window also covers the region in which the artery walls might be present due to the distension, the values in the first column of the segmented window representing the rf signals of the echoes, is auto-correlated across the successive frames to obtain the distension of the artery walls as explained in the Equations 4.3 - 4.6. Having known the shift of the artery wall across every frame, the change in the lumen diameter of the artery at each frame can be calculated using the Equations 4.15 and 4.16. The location of the proximal inner wall along depth is given by $\mathrm{P}_{\text {inner }}$, and the displacement of the proximal inner wall is given by $\tau_{p w}(t)$, similarly, for the distal inner wall, it is given by $D_{\text {inner }}$ and the displacement of the distal inner wall is given by $\tau_{d w}(t)$. The change in the lumen diameter at an instance of time is given by Equation 4.16.

$$
\Delta D(t)=\left(\mathrm{D}_{\text {inner }}+\tau_{d w}(t)\right)-\left(\mathrm{P}_{\text {inner }}+\tau_{p w}(t)\right)
$$

The systolic and diastolic diameter can be estimated by taking the average of the diameter values at the peak and average of the diameter values at valley, respectively, observed in 
$\Delta D(t)$. The peak and valley of the curve plotted using $\Delta D(t)$ is obtained by finding all local maxima and local minima by a simple comparison of the neighboring values. The peak and valley detection functions are implemented with the help of the SciPy library [82]. 


\section{Chapter 5: Results and discussion}

This chapter discusses the performances of the proposed algorithms for artery detection and lumen diameter tracking. The performance comparison of the proposed ML algorithms to classify the segmented windows for artery detection under two different experimental conditions (phantom-based and human in-vivo) is discussed in Section 4.1. The details of the experimental conditions (phantom experiments and human in-vivo) were explained in Section 3.2. The results of the lumen diameter tracking obtained using the correlationbased diameter change estimation technique is discussed in Section 4.2.

\subsection{Detection of artery wall regions}

Locating the artery wall without the help of a B-mode image guidance is often very difficult; hence, it is useful to have an algorithm to detect the artery walls from an M-mode rf signal acquired with a single UT system for which B-mode image is not available. Since there is no benchmark available to identify the effectiveness of the considered features, proposed machine learning algorithms are compared against each other. The performance of machine learning-based methods for artery wall region detection depends on the appropriate selection of features. Chosen machine learning algorithms considered for comparison in this thesis are Linear Discriminant Analysis (LDA), K- Nearest Neighbor $(\mathrm{KNN})$, Random Forest (RF), and Extreme Gradient Boosting (XGB), as explained in

Section 4.1.4. In addition to the machine learning algorithms mentioned above, the performance of the Convolutional Neural Network $(\mathrm{CNN})$ that extracts the features automatically from the segmented window is also compared. The metrics used to compare 
the performance of different algorithms are Accuracy, F1 score, Area under the ROC curve, and the Precision-Recall curve.

\subsubsection{Result for Phantom A}

This section provides an analysis of classification results for Phantom A with PICUS clinical ultrasound imaging system. The classification algorithms are trained and validated, using all the manually extracted features in slow-time and fast-time described in Section 4.1.2, except the CNN which extracts the features automatically, on 181116 samples and tested on 11232 samples, respectively. The samples for training, validation and testing are obtained by using the ground truth creation technique (explained in Section 4.1.1) on the ultrasonic data acquired from Phantom experiments (explained in Section 3.2.1.2 ). The comparison between different classifiers in terms of accuracy and the F1 score is given in Table 5.1 and Table 5.2.

Table 5.1 Accuracy of different machine learning algorithms for

\section{Phantom A.}

\begin{tabular}{|l|l|l|l|l|l|}
\hline & LDA & KNN & RF & XGB & CNN \\
\hline Accuracy & 0.987 & 0.997 & 0.969 & 0.976 & 0.703 \\
\hline
\end{tabular}


Table 5.2 F1 score of different machine learning algorithms for

Phantom A.

\begin{tabular}{|c|c|c|c|c|c|}
\hline & LDA & KNN & RF & XGB & CNN \\
\hline No boundary & 0.99 & 0.99 & 0.98 & 0.98 & 0.96 \\
\hline $\begin{array}{c}\text { Proximal } \\
\text { wall }\end{array}$ & 1.00 & 1.00 & 0.94 & 1.00 & 0.65 \\
\hline Distal wall & 0.97 & 1.00 & 0.97 & 0.95 & 0.12 \\
\hline
\end{tabular}

The accuracy of the classifiers with all the given set of slow-time and fast-time features is given in Table 5.1. Since evaluating the classifier based on accuracy can be misleading, the F1 score of the classifiers for each of the classes are also compared. From Table 5.2, it is seen among the five classifiers tested that the CNN classifier has lowest F1 score for all the three classes, particularly for the distal wall class. As explained in Section 3.2.1.2, Phantom A is an ideal case without scattering noises. The phantom A experimental setup involves a polymer tube simulating the artery wall surrounded by water. Hence, the classifiers perform well with an overall accuracy above 0.97 in general. It is seen that CNN has the lowest skill among the classifiers considered. The reason for this lowest performance of $\mathrm{CNN}$ could be explained by observing its normalized confusion matrix as shown in Figure 5.1. 
(A)
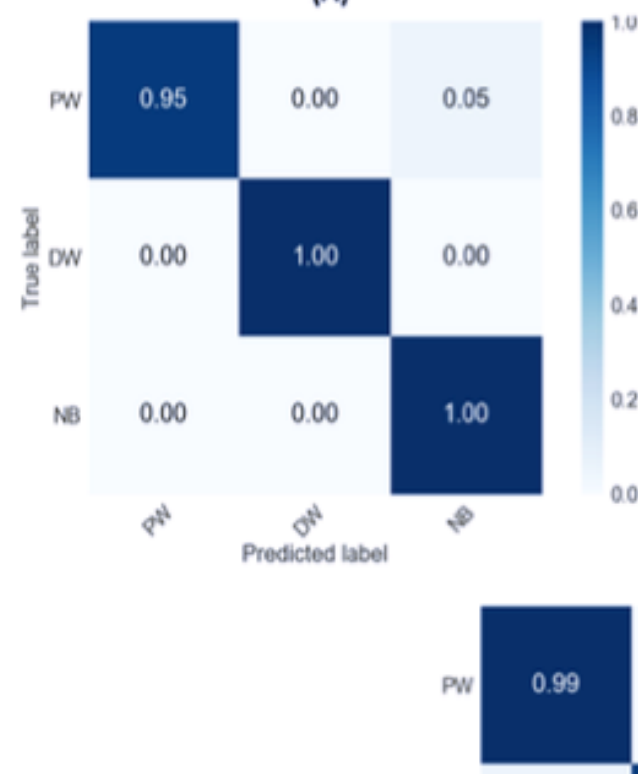

$\frac{\frac{\Phi}{9}}{\frac{9}{2}} \mathrm{DW} \quad 0.05$

(D)

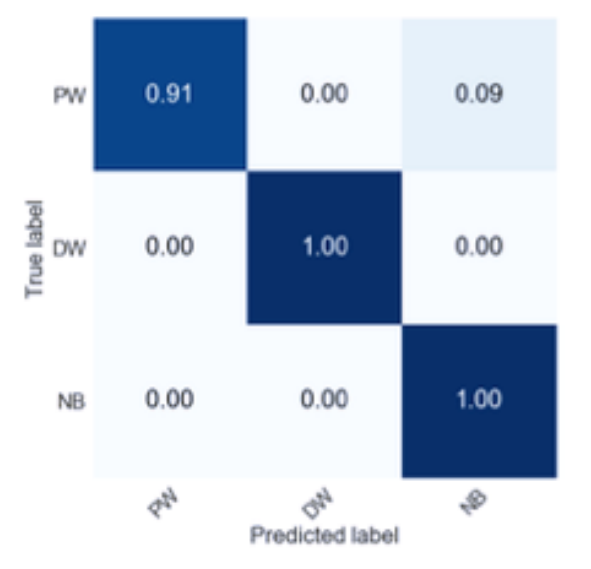

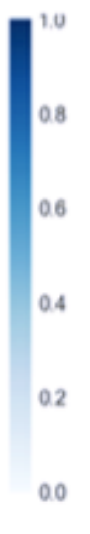

(C)
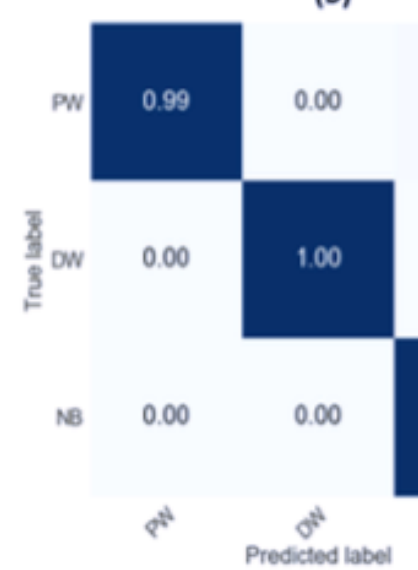

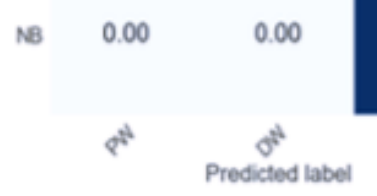

(c)

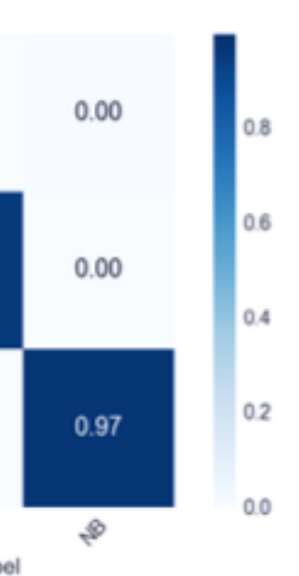

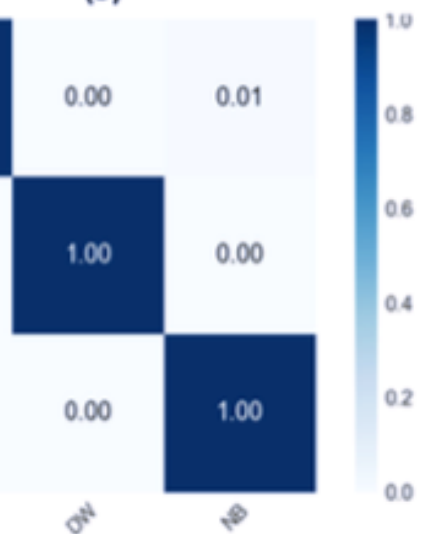

(B)

00

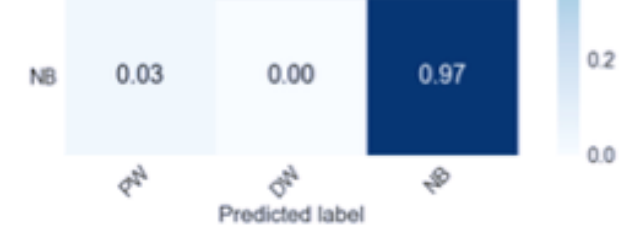

E)

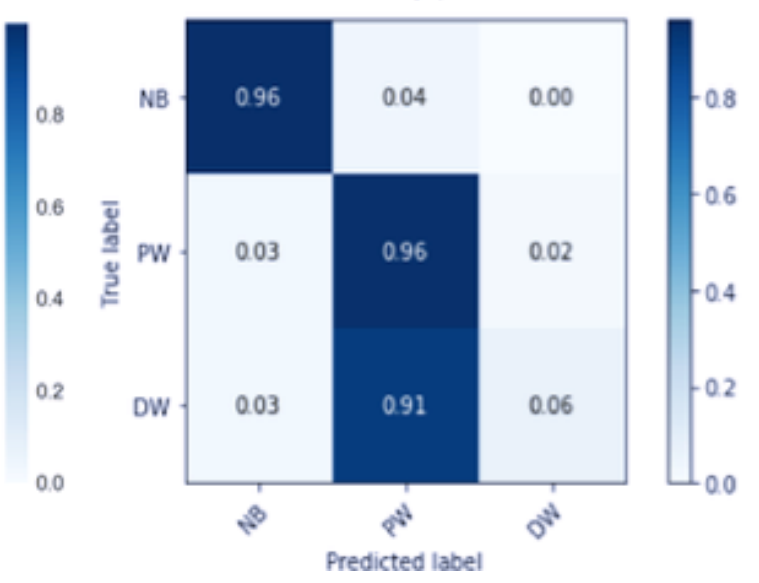

Figure 5.1 Normalized confusion matrix of (A) LDA, (B) KNN, (C)

RF, (D) XGB and (E) CNN for Phantom A. 
It is seen from Figure 5.2 (B) for the CNN result, that $91 \%$ of the segmented windows labeled as a distal wall are misclassified as a proximal wall. The misclassification could be because of two factors: no scattering noises from the water surrounding the polymer tube and the manually induced motion of the polymer tube, as discussed as follows. In addition, the CNN does not use the proposed slow-time and fast-time features extracted manually to differentiate the proximal and distal walls, discussed in Section 4.1.2. For a given segmented window labeled as proximal or distal wall, there is water both above and below the artery wall.

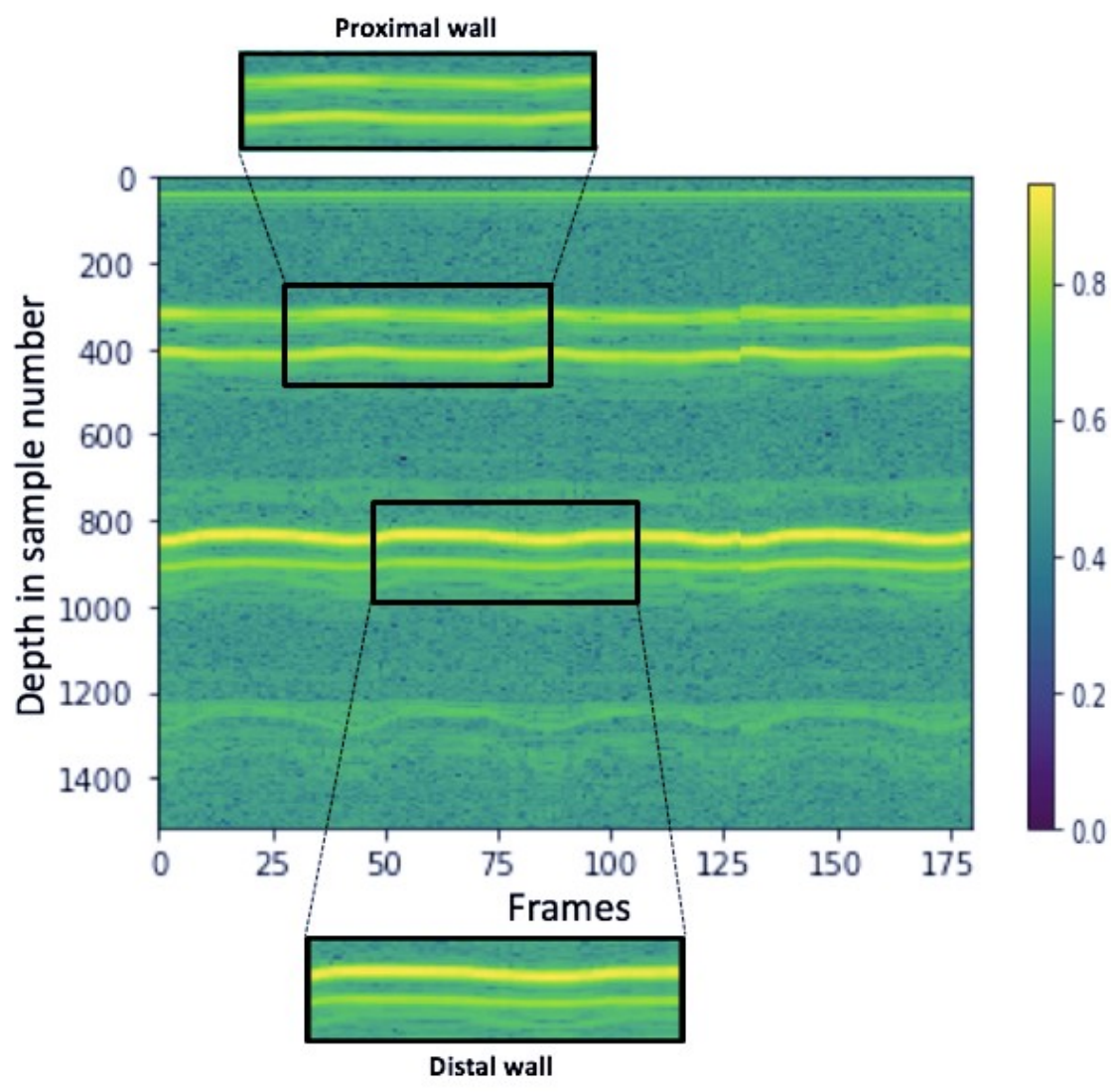

Figure 5.2 Segmented windows of proximal and distal wall of Phantom A at different instances of time. 
Figure 5.2 shows the M-mode rf signals obtained with Phantom A. It is observed from Figure 5.2 that the artery wall motion induced by a syringe is sinusoidal, unlike the motion induced by the actual human cardiac cycle. Thus, the segmented windows containing the motion of the proximal wall and the distal wall at different time instances are similar. This could explain the misclassification of the distal wall as a proximal wall by the CNN classifier. The misclassification of the proximal wall as no boundary region is due to the reverberation artifact explained in Section 3.2.1.2.

The machine learning algorithms based on the manually extracted features, perform better in the case of Phantom A, as the segmented windows are distinguished primarily based on the amplitude-based features of the rf signal (energy, maximum amplitude, and minimum amplitude) considered. The manually extracted features capture the change in the amplitude of the observed rf signals. The rf signal echoes from the segmented region containing the proximal wall has a higher amplitude as compared to the distal wall as the distal wall is further away from the sensor. This predominantly helps the classifier using the manually extracted features to perform better in the case of Phantom A.

\subsubsection{Result for Phantom B}

This section provides an analysis of the classification results for Phantom B with PICUS clinical ultrasound imaging system. The Phantom B experimental setup closely resembles the in-vivo human conditions, and thus more realistic experimental environment than 
Phantom A. Therefore, the results obtained for the classifiers are expected to be similar to those obtained for the in-vivo human conditions.

The classification algorithms are trained and validated on 321165 samples and tested on 111384 samples. The samples for training, validation and testing are obtained by using the ground truth creation technique (explained in Section 4.1.1) on the ultrasonic data acquired from Phantom B experiment (explained in Section 3.2.1.3). The comparison between different classifiers in terms of accuracy and the F1 score is given in Table 5.3 and Table 5.4 .

Table 5.3 Accuracy of different machine learning algorithms for Phantom B.

\begin{tabular}{|l|c|c|c|c|c|}
\hline & LDA & KNN & RF & XGB & CNN \\
\hline Accuracy & 0.902 & 0.920 & 0.754 & 0.809 & 0.970 \\
\hline
\end{tabular}

Table 5.4 F1-score of different machine learning algorithms for Phantom B.

\begin{tabular}{|c|c|c|c|c|c|}
\hline & LDA & KNN & RF & XGB & CNN \\
\hline Proximal wall & 0.88 & 0.91 & 0.94 & 0.96 & 0.98 \\
\hline Distal wall & 0.71 & 0.80 & 0.51 & 0.60 & 0.94 \\
\hline No boundary & 0.95 & 0.95 & 0.80 & 0.85 & 0.95 \\
\hline
\end{tabular}


On observing the performance of the different classifiers from Table 5.3 and 5.4, it is seen that the $\mathrm{CNN}$ outperforms the rest of the classifiers. The automatic feature extraction of $\mathrm{CNN}$ is better than the manually extracted features. Among the classifiers that used the manually removed features, $\mathrm{KNN}$ is the best classifier for the classes of the distal wall and no boundary. 


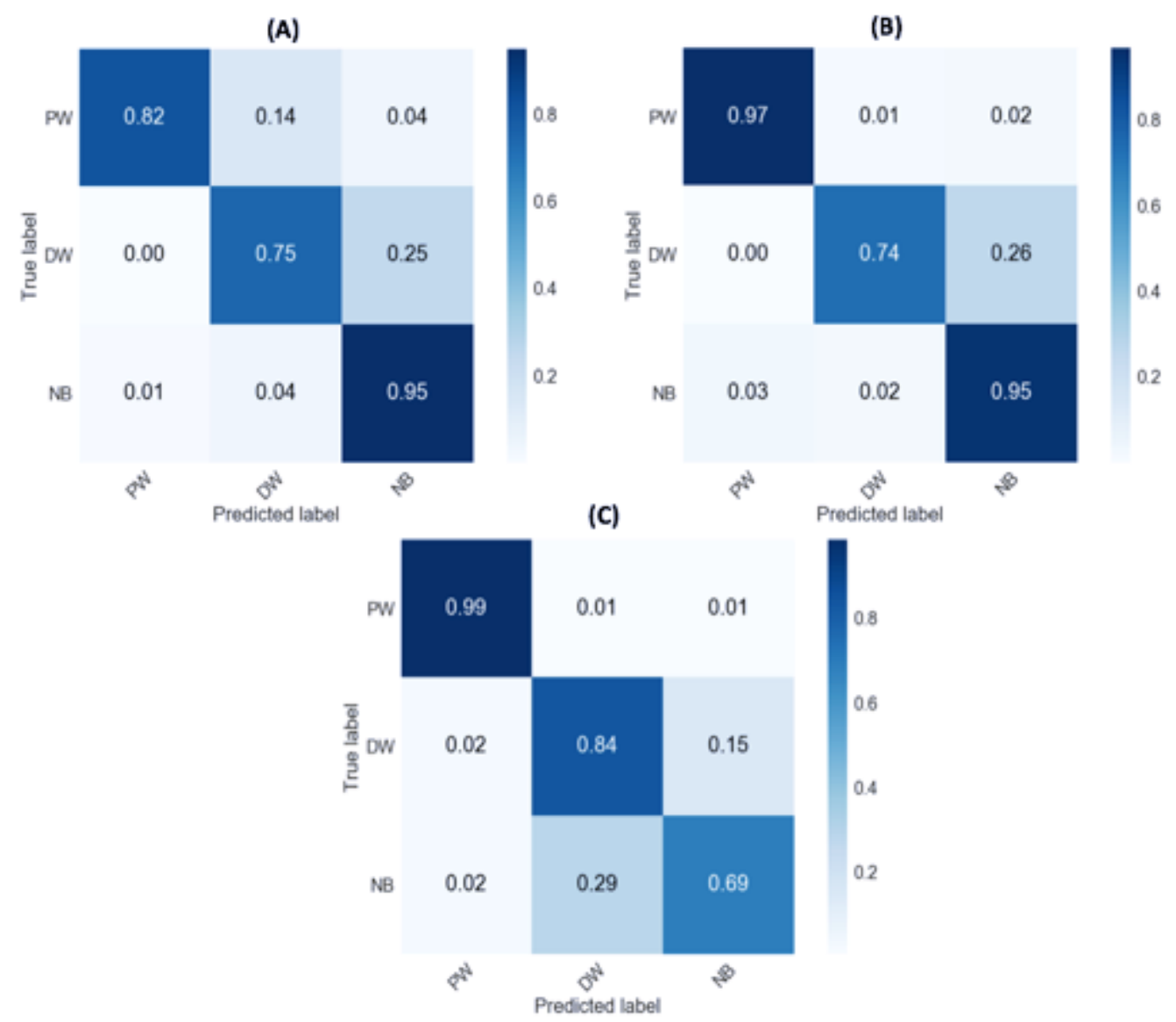

(D)
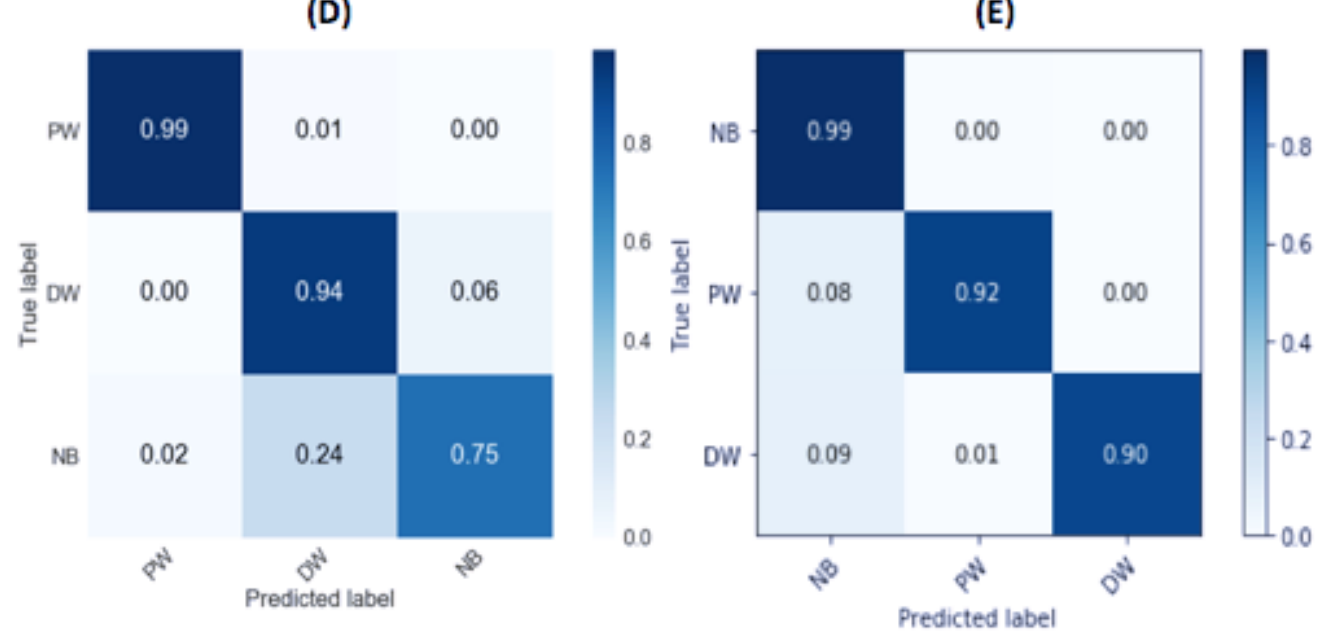

Figure 5.3 Normalized confusion matrix of (A) LDA, (B) KNN, (C)

RF, (D) XGB and (E) CNN for Phantom B. 
It is observed from the Table 5.3 and Table 5.4, CNN performs better in the case of Phantom B. The difference in the performance of CNN when compared with its performance for Phantom A may be attributed to the experimental set up of Phantom B. Unlike the Phantom A in which water is both in and around the polymer tube, Phantom B has the tissue-mimicking gelatine material outside and water inside the polymer tube.

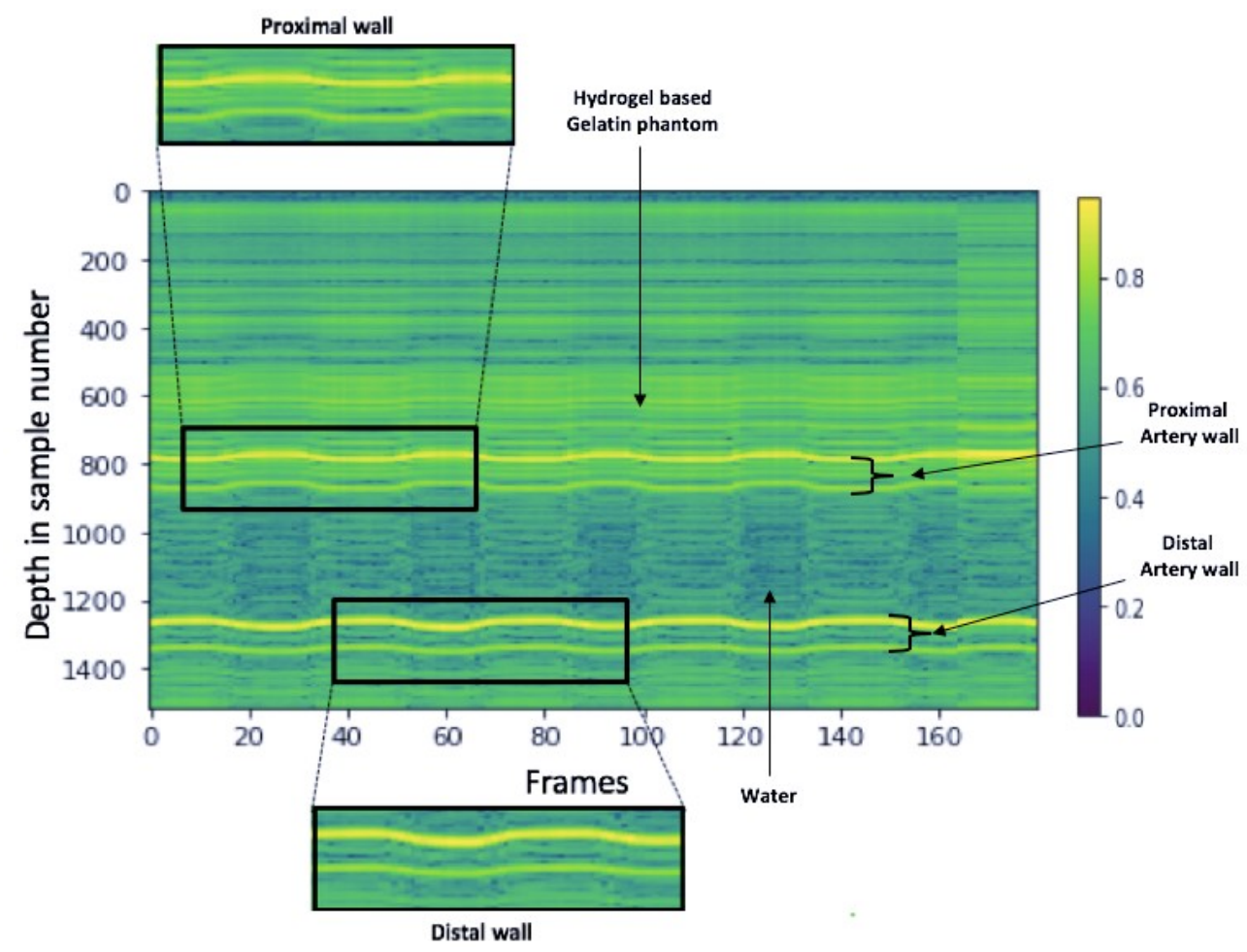

Figure 5.4 Segmented windows of proximal and distal wall of

Phantom $B$ at different instances of time.

Figure 5.4 shows the M-mode rf signals obtained with Phantom B. It is seen from Figure 5.4 that the segmented window of the proximal wall (the upper rectangular box) has scattering echoes from the carbon particles in the gelatin material above the artery wall and 
less scattering echoes from water below the artery wall. In the case of a distal wall (the lower rectangular box), there is water above the artery wall and gelatin material below the artery wall. The scattering echoes from the surrounding material of the polymer tube also exhibit a displacement due to the induced motion of the polymer tube. Since the tissuemimicking gelatine phantom is viscoelastic, the movement of the polymer tube also moves the surrounding hydrogel material surrounding the polymer tube, as observed in Figure 5.4. However, no motion pattern is observed in the M-mode image of the water inside the polymer tube simulating artery due to the polymer tube motion. Thus, the region of gelatin material above and water below the artery wall is successfully classified as the proximal wall, while the region of gelatin material below and water above the artery wall is classified as the distal artery wall. The KNN classifier performs better than the rest of the classifiers overall, in the case of the manually extracted features. It is observed from the normalized confusion matrix of the KNN in Figure 5.3, that the classifier misclassifies 0.26 of the distal wall as no boundary region. This is reflected as a lowest F1 score of 0.8 for the distal wall among the classes. It is noted that misclassifications of the distal wall region as no boundary and no boundary as the distal wall are observed in all classifiers designed with manually extracted features. The main reason for this misclassification of the distal wall could be explained from the observation of the appearances of the considered features in Figure 5.6. Due to more ultrasound attenuation at deeper regions of the phantom, the rf signal amplitude observed at the distal wall is smaller than those at the proximal wall and is similar to those of the scattering echoes below the distal wall. 


\subsubsection{Result for in-vivo human subjects}

For the in-vivo human subjects, the proposed algorithms of artery wall region detection was tested with the ultrasonic data obtained with PICUS clinical ultrasound system in Section 5.1.3.1 and WUS system in Section 5.1.3.2. The experiment with a human subject was approved by the Carleton University ethics board.

\subsubsection{PICUS clinical ultrasound imaging system}

This section provides an analysis of classification results for in-vivo human experiments with PICUS clinical ultrasound imaging system. The classification algorithms are trained and validated on 131692 samples and tested on 22440 samples. The samples for training, validation and testing are obtained by using the ground truth creation technique (explained in Section 4.1.1) on the ultrasonic data acquired from human in-vivo experiment (explained in Section 3.2.2). The human in-vivo ultrasonic data were acquired from 8 human subjects. Among these ultrasonic data, the data from 6 subjects were used for training, and data from 2 subjects were used for testing. Thus, the test data to the classifier is completely masked during the training and validation process. The samples are The performance of the classifier on the unseen test data is presented in Table 5.5 and Table 5.6. 
Table 5.5 Accuracy of the machine learning algorithms for in-vivo human subject with PICUS system.

\begin{tabular}{|l|c|c|c|c|c|}
\hline & LDA & KNN & RF & XGB & CNN \\
\hline Accuracy & 0.846 & 0.796 & 0.818 & 0.874 & 0.889 \\
\hline
\end{tabular}

Table 5.6 F1-score of the machine learning algorithms for in-vivo human subject with PICUS system.

\begin{tabular}{|c|c|c|c|c|c|}
\hline & LDA & KNN & RF & XGB & CNN \\
\hline Proximal wall & 0.74 & 0.76 & 0.64 & 0.77 & 0.76 \\
\hline Distal wall & 0.66 & 0.70 & 0.73 & 0.74 & 0.86 \\
\hline No boundary & 0.91 & 0.91 & 0.89 & 0.92 & 0.92 \\
\hline
\end{tabular}

The results obtained shows that CNN classifier performs better than the rest of the classifiers in terms of accuracy with $88.9 \%$. On observing the F1 -score for each of the classes (no boundary, proximal wall and distal wall), the CNN classifier performs better than or equal to the other classifiers considered for the cases of distal and no boundary regions. The drop in the F1-score for the proximal class in case of CNN is explained below. The performance of the classifiers, in terms of accuracy, with the human subjects is not as good as that obtained with Phantom B as the maximum accuracy obtained in the case of human subjects is only $88.9 \%$ ( $97 \%$ in case of $\mathrm{CNN})$. This is probably due to the differences in the cardiac cycle patterns and the thickness of the artery wall among the 
individuals. Also, there may be motion artifact in the ultrasonic rf signal due to the ultrasonic probe motion during the ultrasound data acquisition. 


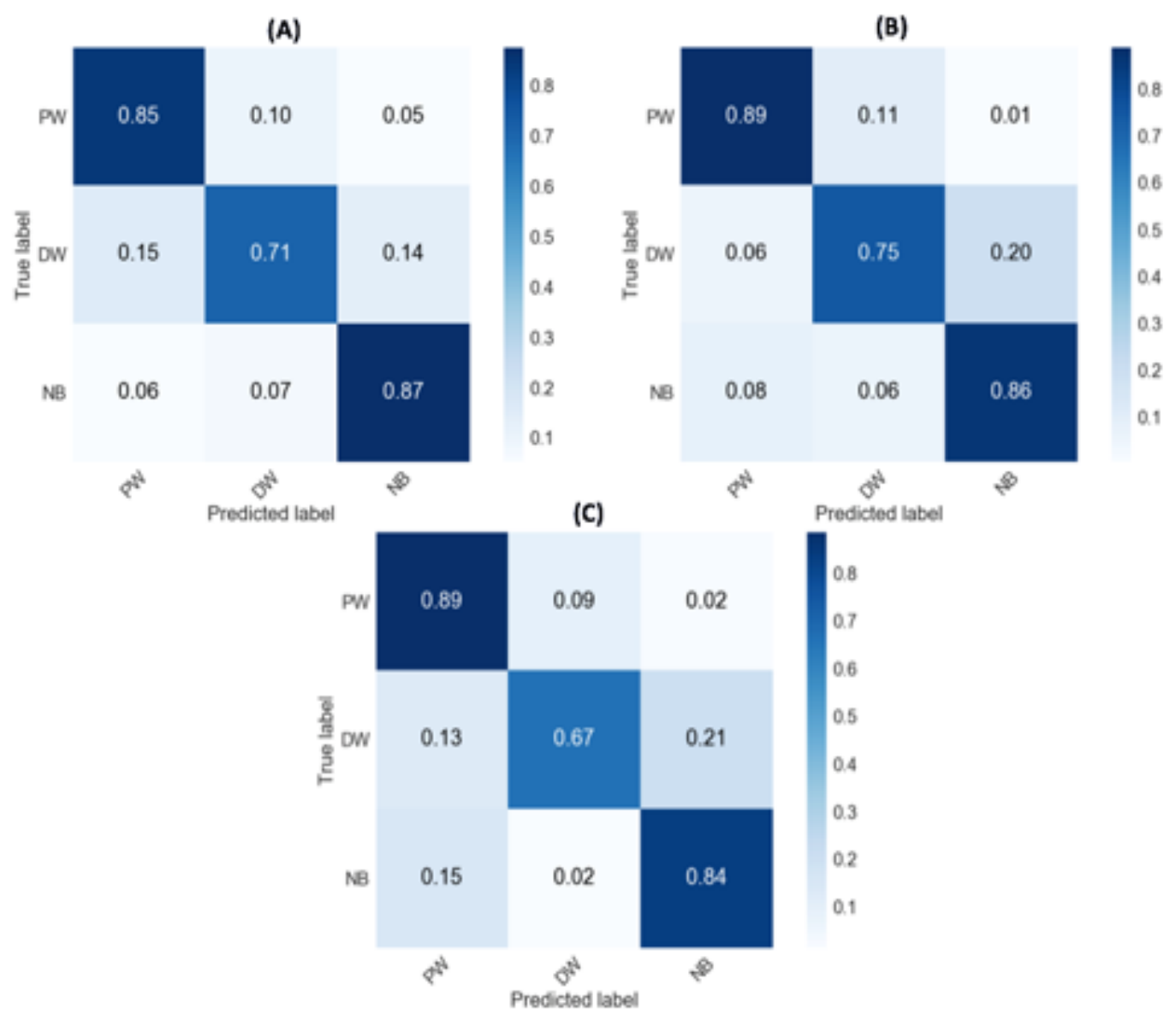

(D)

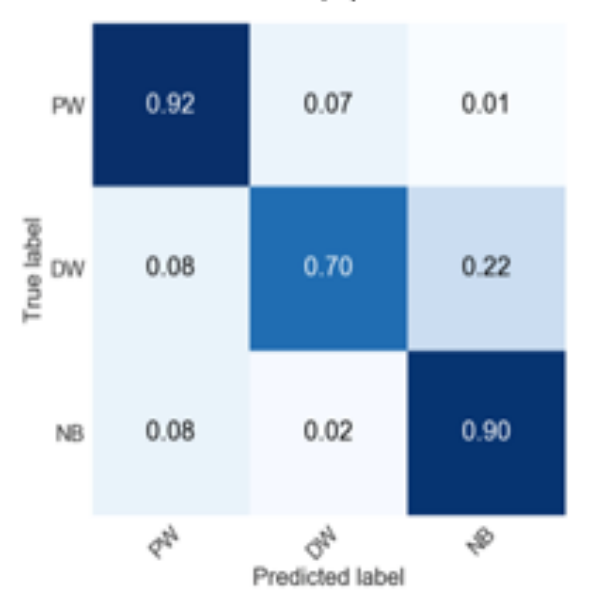

(E)

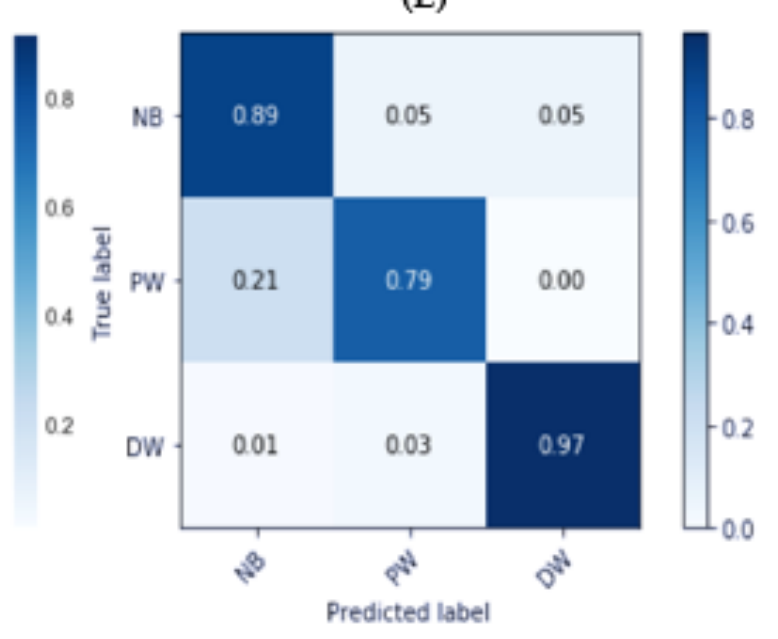

Figure 5.5 Normalized confusion matrix of (A) LDA, (B) KNN, (C) RF, (D) XGB and (E) CNN for in-vivo human subject with PICUS system. 
Figures 5.5 show the normalized confusion matrix of the classification results. It is seen in Figures 5.5, the KNN and the XGB perform better than the rest of the algorithms using the manually extracted features. 


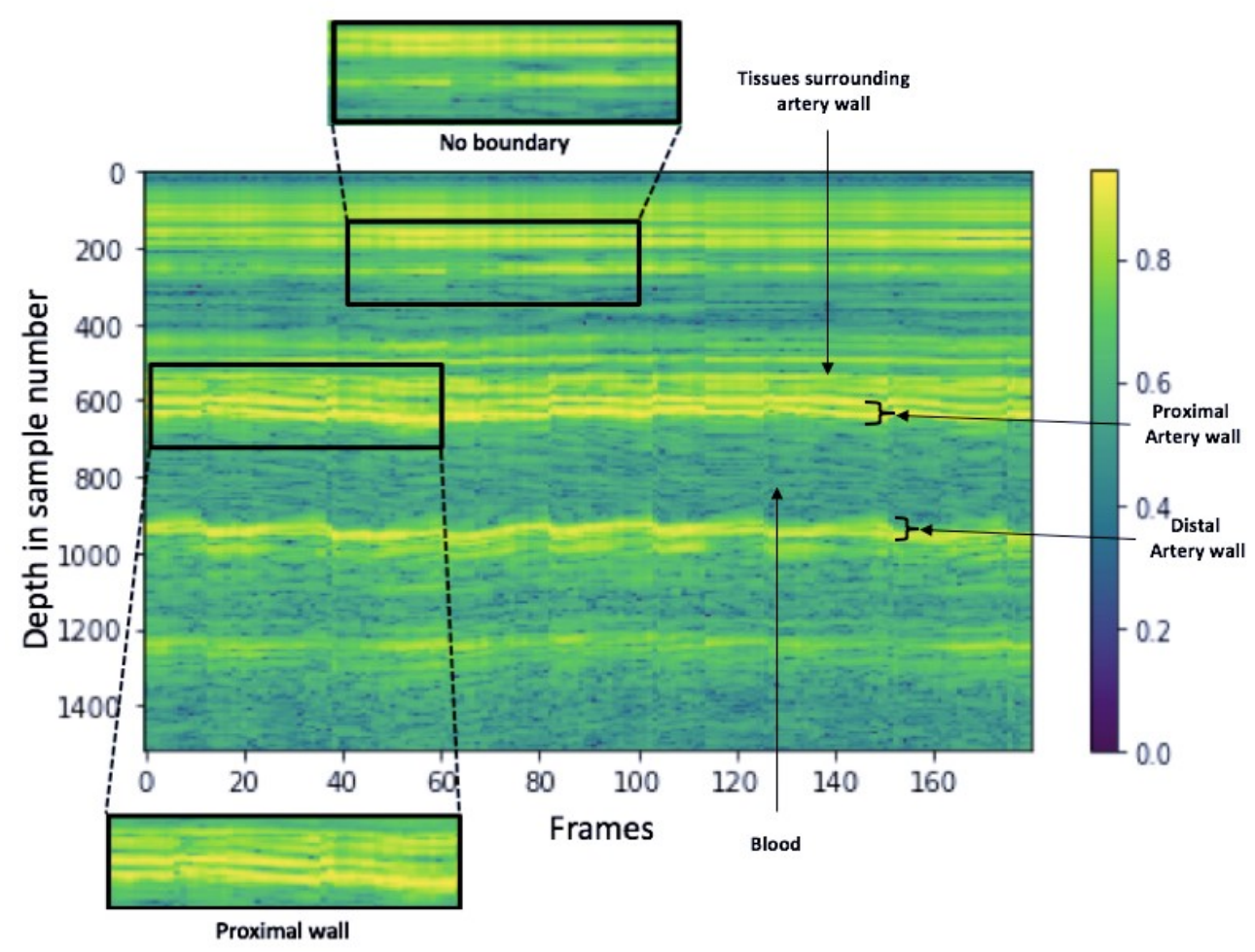

Figure 5.6 Segmented windows of proximal and no boundary of invivo human subject with PICUS system at different instances of time.

From Figure $5.5(\mathrm{E})$, it is observed that the $\mathrm{CNN}$ misclassifies the proximal wall region as the no boundary region. The misclassification may be due to the fact that the motion of the proximal wall is less than that of the distal wall as observed in Figure 5.6. Due to the less motion of the proximal artery wall during the cardiac cycle, the region containing the proximal wall is more similar to the segmented window containing the no boundary region. The restricted motion of the upper artery wall may be due to the pressure applied on the skin surface by the PICUS clinical ultrasound probe, the nonuniformity of the stiffness of the soft tissues surrounding the artery, and/or nonuniformity of the stiffness of the artery wall. Overall, it is seen that the CNN classifier outperforms the rest of the classifiers in two 
of the experimental setups (i.e., Phantom B and in-vivo human subject). Therefore, the $\mathrm{CNN}$ is tested for the ultrasonic data obtained with the in-vivo human subjects with WUS system in the following section.

\subsubsection{Wearable ultrasound sensor (WUS) system}

This section provides an analysis of classification results for in-vivo human experiments with the WUS system. Based on the performance of different proposed algorithms on the ultrasound rf signal acquired with the PICUS clinical imaging system, the CNN consistently performs better than the rest of the algorithms for Phantom B and human subjects. Thus, the rf signal acquired with in-vivo human subjects using the WUS system is used to train and test the $\mathrm{CNN}$ classifier. The $\mathrm{CNN}$ is trained and validated on 16081 segmented widow samples, while it is tested on 4030 samples. The difference in the data format of the WUS system and PICUS clinical ultrasound system is explained in Section 3.1. As described in Section 4.1.1, the size of the segmented window in the case of the WUS system is selected to be 800 sample points in fast time (depth) $\times 2000$ frames in the slow time. The size of the input image (segmented window) fed to the $\mathrm{CNN}$ is resized into 200 sample points $\times 60$ frames using sci-kit learn pre-processing image library [92]. It is necessary to resize the segmented window to a smaller size, to strike a balance between the number of the parameters in a $\mathrm{CNN}$ to be trained and the total number of segmented windows available for training. Due to a low signal to noise ratio of the rf ultrasound signals obtained with the WUS system, it was difficult to obtain more samples with the WUS system. Hence, the segmented window is resized to reduce the number of parameters to be 
trained. The architecture of CNN used in the case of ultrasound rf signal acquired with WUS is given in Table 4.1 .

Table 5.7 Accuracy and F1-score of CNN for in-vivo human subject with WUS system.

\begin{tabular}{|c|c|c|}
\hline & F-1 Score & Accuracy \\
\hline Proximal wall & 0.98 & \multirow{2}{*}{0.883} \\
\hline Distal wall & 0.67 & \\
\hline No boundary & 0.71 & \\
\hline
\end{tabular}

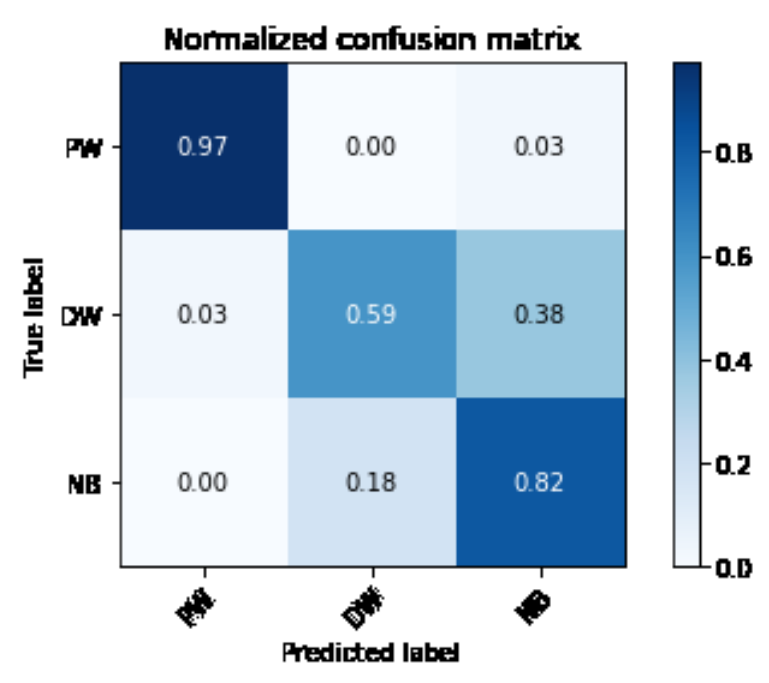

Figure 5.7 Normalized confusion matrix of CNN for in-vivo human subject with WUS system.

(In the following discussion present the values in Table 5.7 and Figure 5.9 and 5.10.) 
From Table 5.7 and Figure 5.7, it is observed that CNN with WUS system has more difficulty in distinguishing between the no boundary region and the distal wall region. Unlike, the case of in-vivo human experiments using a PICUS clinical ultrasound probe, the WUS does not restrict the movement of the upper artery wall (shown in Figure 3.16); hence, the proximal wall is not misclassified to be a no boundary window. Misclassification of no boundary region as distal wall and vice-versa happens due to the small amplitude (weak) of the echoes. The precision-recall curve and the Receiver Operating Characteristic (ROC) curve for all the classifiers for all experimental conditions are given in the Appendix. Based on the results observed, the manually selected features are able to perform with an accuracy that is not very far off from the automatically extracted features, this shows that the combination of the manually extracted features are able to create distinguishable patterns in the higher dimensions (combination of the features) that are distinguished with the help of a machine learning classifier. But the performance could have been improved by including the frequency-based features.

\subsection{Tracking of arterial walls and lumen diameter estimation}

The segmented region containing the proximal and the segmented region containing the distal wall of the artery, are identified using the artery detection technique described in Section 4.1, and the results are discussed in Section 5.1. In order to locate and track the lumen diameter, the lumen diameter is identified using the peak detection technique described in Section 4.2.1. Once the inner artery walls (proximal inner wall and distal inner wall) are identified, and the distance between them is determined using Equation 4.15. The

cross-correlation based technique explained in Section 4.2.2 is used to track the 
displacements of the proximal and distal walls. Since the lumen diameter and the movement of the proximal and the distal walls are estimated, the change in the lumen diameter of the artery is estimated by Equation 4.16. The results of the change in artery lumen diameter due to the cardiac cycle obtained for Phantom A, Phantom B, and in-vivo human subjects are presented in this section.

\subsubsection{Result for Phantom A}

(A)

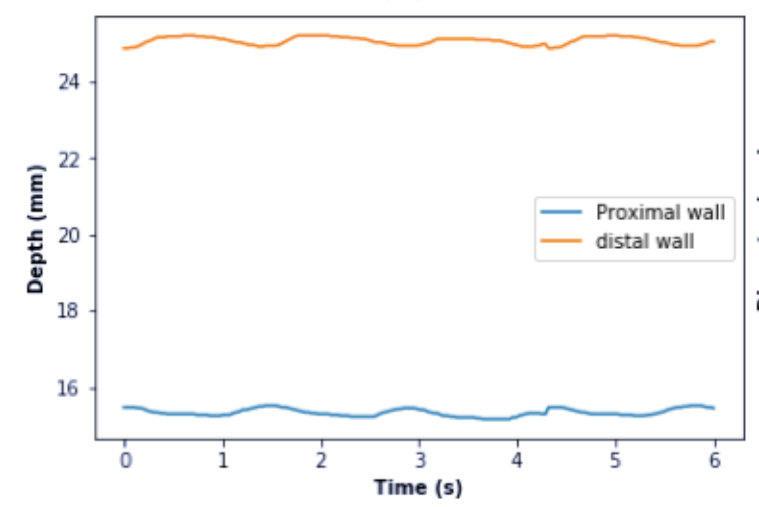

(B)

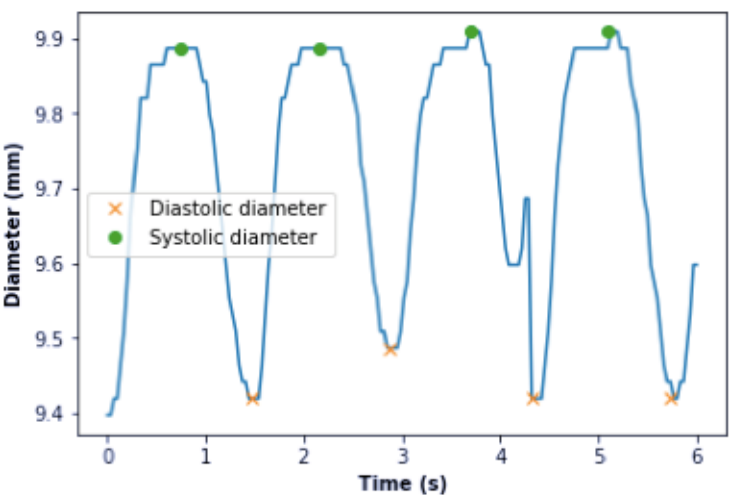

Figure 5.8 (A) Displacements of the proximal and distal walls of polymer tube at their corresponding depths, and (B) change of its inner diameter of Phantom A with respect to the measurement time.

Figure 5.8 (A) shows the displacement pattern of the proximal wall and the distal wall at their corresponding depths estimated by the KNN classifier using the ultrasound data of Phantom A acquired by PICUS clinical ultrasound imaging system. It can be observed that 
the proximal and the distal wall move opposite to each other; this is due to the pumping of the water into and out from the polymer tube to simulate the cardiac cycle. Figure 5.8 (B) shows the tube inter diameter obtained from the result in Figure 5.8 (A). In this phantom simulation experiment, the time interval between two consecutive maxima or minima observed in Figure 5.8 (B), is called a cycle hereafter. Further, the change in the inner diameter of the polymer tube is not seen to be uniform; this is probably because of the slightly different amount of water manually pumped into and out from the tube in each cycle during the experiment, even though efforts were taken to make sure the amount of water to be the same. The maximum difference between the minimum inner diameter of the tube due to the pumping error is observed to be around $60 \mu \mathrm{m}$ between the $2.9 \mathrm{sec}$ and $4.5 \mathrm{sec}$.

Further, the sudden diameter increase and decrease of about $100 \mu \mathrm{m}$ observed around 4.2 sec in Figure 5.8 (B) is probably due to the water pumping error, as explained in Section 3.2.1.2. It can be concluded from Figure 5.8 that the proposed algorithm to estimate the change in the inner diameter can track the changes of the inner diameter even when there is an inconsistency of the amount of the water pumped. The peak and valley detection algorithm ignores the false valley generated as it only detects peaks and valley that is at least 0.45 seconds away from each other. 
Table 5.8 Minimum and maximum inner diameter of the polymer tube estimated for Phantom A.

\begin{tabular}{|c|c|c|c|}
\hline Cycle number & $\begin{array}{c}\text { Minimum diameter } \\
\text { in } \mathbf{~ m m}\end{array}$ & $\begin{array}{c}\text { Maximum diameter } \\
\text { in } \mathbf{~ m m}\end{array}$ & $\begin{array}{c}\text { Inner tube diameter at } \\
\text { rest } \\
\text { in } \mathbf{~ m m}\end{array}$ \\
\hline 1 & 9.420 & 9.886 & \multirow{2}{*}{9} \\
\hline 2 & 9.487 & $9.886 \pm 0.04$ \\
\hline 3 & 9.420 & 9.908 & \\
\hline 4 & 9.420 & 9.908 & \\
\hline Mean \pm Std & $9.437 \pm 0.028$ & $9.898 \pm 0.011$ & \\
\hline
\end{tabular}

The minimum and the maximum diameter for each cycle estimated using the technique specified in Section 4.2.2 is given in Table 5.8. The actual inner diameter of the polymer tube along the axis of measurement, is measured with the help of a Vernier caliper (measurement resolution $0.01 \mathrm{~mm}$ ) at four different places along the tube. The inner diameter of the tube along the axis of measurement is measured to be $9.48 \pm 0.04 \mathrm{~mm}$. The difference observed in the actual inner diameter of the artery measured using the Vernier caliper is due to the slight deformation of the tube during the construction of the 
experimental setup.It is to be noted that the standard deviation (std) calculated using the proposed trechnique includes both the measurement error and the experimental error introduced due to the uneven pumping of water into the polymer tube simulating the artery.

\subsubsection{Result for Phantom B}

(A)

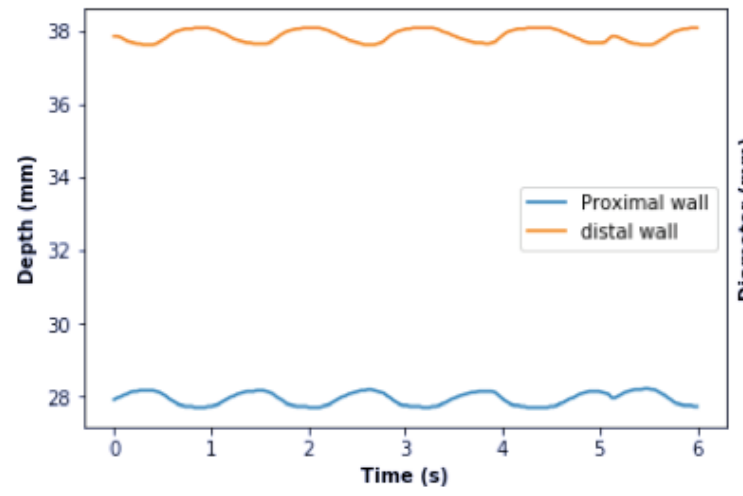

(B)

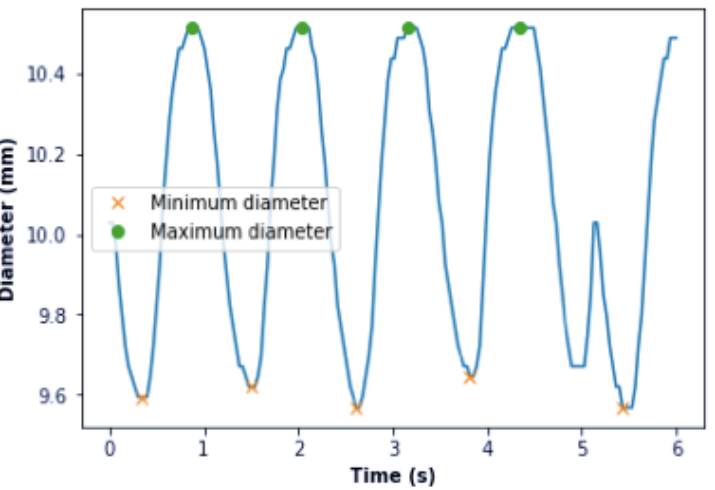

Figure 5.9 (A) Displacements of the proximal and distal walls of polymer tube at their corresponding depths, and (B) change of its inner diameter of Phantom B with respect to the measurement time.

The Figure 5.9 (A) shows the distension pattern of the proximal wall and the distal wall at their corresponding depths estimated by the CNN classifier using the ultrasound data of Phantom B acquired by PICUS clinical ultrasound imaging system. The distension of the proximal and the distal wall is due to manual pumping of the water into the polymer tube surrounded by the gelatine phantom, to simulate the cardiac cycle. Figure 5.9 (B) shows 
the change in the inner diameter of the phantom tube simulating the artery walls. From Figure 5.9 (B), it is observed that the pattern at which the distension occurs is not precisely similar to the distension of the artery walls during a cardiac cycle. The duration of the systolic and the diastolic pressures that cause the distension are different due to the manual pumping of the water into and out from the tube. The experimental setup for Phantom B is more realistic to mimic artery surrounded by the soft tissues with scatterers, simulating invivo human subjects, and thus it is more appropriate to evaluate the algorithm proposed in Chapter 4 than phantom A. During the process of pumping water into and out from the polymer tube, efforts were taken to make sure the displacement caused due to the manual pumping of the polymer tube is the same for every cycle, but just like Phantom A, there is a difference observed in the order of $90 \mu \mathrm{m}$ between $2.8 \mathrm{sec}$ and $3.7 \mathrm{sec}$. Similar to tracking the inner diameter of Phantom A, it can be concluded from Figure 5.9 that the proposed algorithm to estimate the change in the inner diameter can track the difference in the inner walls. 
Table 5.9 Minimum and maximum diameter estimated for

Phantom B.

\begin{tabular}{|c|c|c|c|}
\hline $\begin{array}{c}\text { Cycle } \\
\text { number }\end{array}$ & $\begin{array}{c}\text { Minimum diameter } \\
\text { in } \mathbf{~ m m}\end{array}$ & $\begin{array}{c}\text { Maximum diameter } \\
\text { in } \mathbf{~ m m}\end{array}$ & $\begin{array}{c}\text { Inner tube diameter at rest } \\
\text { in } \mathbf{~ m m}\end{array}$ \\
\hline 1 & 9.593 & 10.513 & \\
\hline 2 & 9.619 & 10.513 & \multirow{2}{*}{$9.55 \pm 0.05$} \\
\hline 3 & 9.568 & 10.514 & \\
\hline 4 & 9.645 & 10.514 & \\
\hline Mean \pm & $9.599 \pm 0.030$ & $10.514 \pm 0.001$ & \\
Std & & & \\
\hline
\end{tabular}

The minimum and the maximum diameter for each cycle estimated using the technique specified in Section 4.2.2 is given in Table 5.9. It is to be noted that the standard deviation includes both the measurement error and the experimental error introduced due to the uneven pumping of water into the polymer tube simulating the artery. While estimating the diameter at the valleys of every cycle, there is a false valley and peak created around $5 \mathrm{sec}$, as shown in Figure 5.9. The peak and valley detection algorithm ignore the false valley generated as it only detects peaks and valley that is at least 0.45 seconds away from each other. On observing the results of phantom experiments, it is seen that the algorithm is able to detect the inner artery wall diameter with the help of the proposed peak detection technique. 


\subsubsection{Result for in-vivo human subjects}

In this section, the results with the in-vivo human subjects for the artery wall tracking and lumen diameter estimation are presented. The segmented region of the artery wall in the acquired ultrasound rf signal was determined by the CNN algorithm since it exhibits the best performance among the chosen ML based algorithms tested in Section 5.1. The results with ultrasonic data acquired by the PICUS clinical ultrasound imaging system and those by the WUS system are presented in Sections 5.2.3.1 and 5.2.3.2, respectively.

\subsubsection{PICUS clinical ultrasound imaging system}

(A)

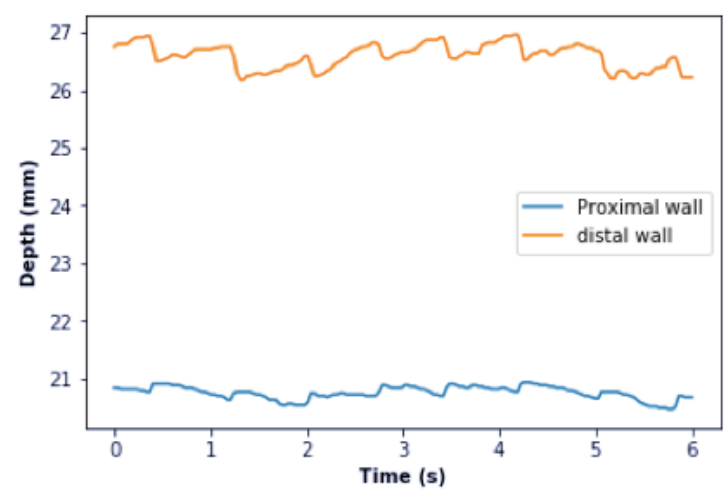

(B)

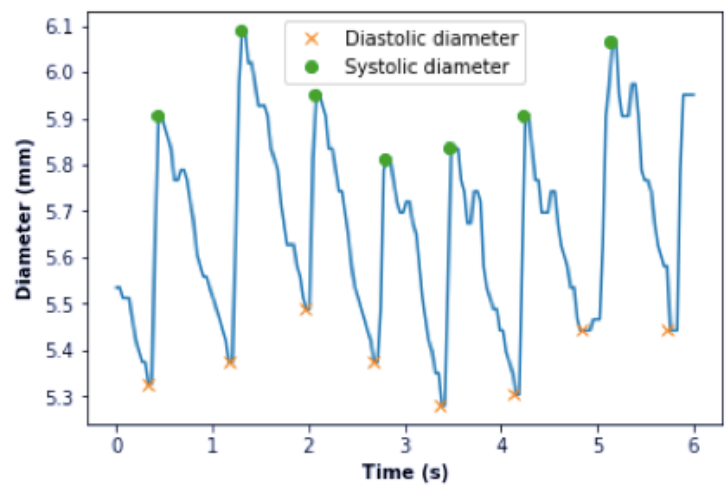

Figure 5.10 (A) Displacements of the proximal and distal walls of carotid artery of human subject at their corresponding depths, and (B) change of its inner diameter with respect to the measurement time. Ultrasound data was acquired with PICUS system. . 
Figure 5.10 (A) shows that the displacement of the proximal wall is smaller than that of the distal wall. The change in the lumen diameter is given in Figure 5.10 (B). In Figure 5.10 (B), the dicrotic notches are observed at each cardiac cycle. The systolic and the diastolic diameters identified by the algorithm proposed in Section 4.2.2 at every cycle are given in Table 5.10.

Table 5.10 Diastolic and Systolic diameter estimated for in-vivo experiments.

\begin{tabular}{|c|c|c|}
\hline Cardiac cycle & Diastolic diameter in mm & Systolic diameter in mm \\
\hline 1 & 5.327 & 5.905 \\
\hline 2 & 5.373 & 6.089 \\
\hline 3 & 5.489 & 5.951 \\
\hline 4 & 5.373 & 5.812 \\
\hline 5 & 5.281 & 5.835 \\
\hline 6 & 5.303 & 5.905 \\
\hline 7 & 5.442 & 6.066 \\
\hline Mean \pm Std & $5.379 \pm 0.069$ & $5.938 \pm 0.099$ \\
\hline
\end{tabular}

From the Table 5.10, it is seen that the systolic and the diastolic diameter observed on the human in-vivo subject is within the range of the common carotid artery diameter range given in [47], [48], [93]. Further, for the same human in-vivo test subject, the lumen diameter of the common carotid artery is measured using a wearable ultrasound transducer 
(WUS) at the location as same as the experiment with the PICUS system, and the results are discussed in the next section.

\subsubsection{Wearable ultrasound sensor (WUS) system}

With the same human in-vivo test subject presented in Section 5.2.3.1, the lumen diameter of the common carotid artery is measured using a wearable ultrasound sensor (WUS) system. Efforts were taken to make sure that the WUS was attached on the same location of the right side of the neck of the human subject where the PICUS probe was attached.

(A)

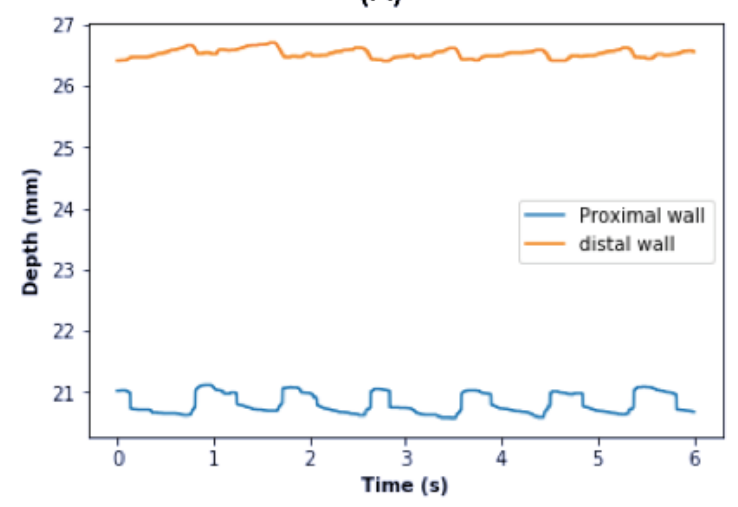

(B)

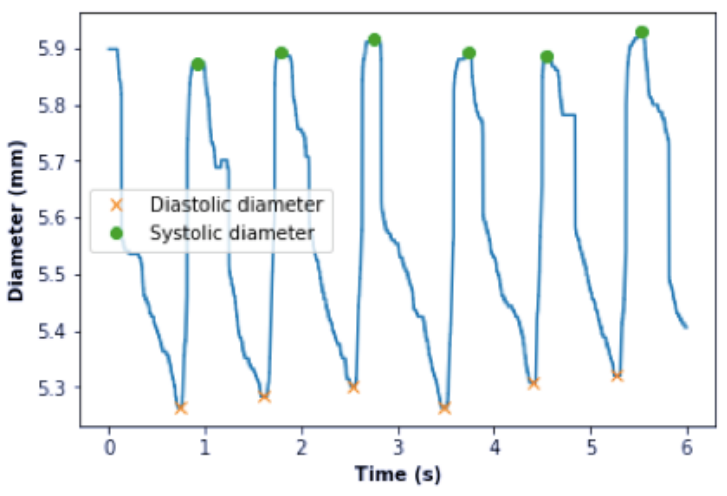

Figure 5.11 (A) Displacements of the proximal and distal walls of carotid artery of human subject at their corresponding depths, and (B) change of its inner diameter with respect to the measurement time. Ultrasound data was acquired with WUS system. . 
Figure 5.11 (A) and (B) show the displacements of the artery walls, and the change in the lumen diameter of the artery for 6 seconds, respectively. The results obtained are comparable to the results obtained with PICUS system discussed in Section 5.2.3.1, as the data is obtained on the same human in-vivo subject. It is seen in the diameter changes obtained by the WUS system shown in Figure 5.11 (B) does not detect the dicrotic notches that were observed in those obtained by the PICUS system shown in in Figure 5.10 (B). This is probably due to that the SNR of the desired artery wall echoes in the ultrasound rf signal acquired by the WUS system are smaller than that of the PICUS system. The systolic and the diastolic diameter obtained using the algorithm explained in Section 4.2.2 is given in Table 5.11.

Table 5.11 Diastolic and systolic diameter estimated for in-vivo using WUS.

\begin{tabular}{|c|c|c|}
\hline Cardiac cycle & Diastolic diameter in mm & Systolic diameter in mm \\
\hline 1 & 5.264 & 5.874 \\
\hline 2 & 5.282 & 5.892 \\
\hline 3 & 5.301 & 5.917 \\
\hline 4 & 5.264 & 5.892 \\
\hline 5 & 5.307 & 5.886 \\
\hline 6 & 5.319 & 5.929 \\
\hline Mean \pm Std & $5.289 \pm 0.021$ & $5.898 \pm 0.019$ \\
\hline
\end{tabular}




\section{Table 5.12 Comparison of mean carotid artery diameters of in-vivo human subject obtained by PICUS system and WUS system.}

\begin{tabular}{|c|c|c|}
\hline & $\begin{array}{c}\text { Mean diastolic diameter } \\
(\mathbf{m m})\end{array}$ & $\begin{array}{c}\text { Mean systolic diameter } \\
(\mathbf{m m})\end{array}$ \\
\hline PICUS & 5.379 & 5.938 \\
\hline WUS & 5.289 & 5.898 \\
\hline Difference & 0.090 & 0.040 \\
\hline
\end{tabular}

Comparing the results in Tables 5.10 and 5.11, it is observed that the systolic and diameter measured using the PICUS system and the WUS system differ from each other, $90 \mu \mathrm{m}$ for the diastolic diameter and $40 \mu \mathrm{m}$ for the systolic diameter, as summarize in Table 5.12. This variation may be due to the experimental condition, different blood pressure of the subject between the experiments with PICUS system and WUS system. Since there is a time interval between the experiments using the PICUS and WUS system, there might be a difference in the blood pressure observed for the same human test subject. The is a difference in the pattern of the cardiac cycle observed between Figure 5.10 and 5.11 is because of two factors: The pressure applied by the transducers (PICUS and WUS) over the artery is different; The signal acquisition from the PICUS system and the WUS system was not at the same instance of time. Hence depending upon the changes in the physiological conditions of the body the pattern of the diameter change observed in Figure 
5.10 and 5.11 is different. Overall, the results obtained from the WUS system is comparable with the results obtained from the PICUS system. 


\section{Chapter 6: Conclusion and Future Work}

This chapter concludes the thesis and provides a summary of the results. Some suggestions for future research are presented, as well.

\subsection{Conclusion}

In this research, automatic artery detection and lumen diameter estimation technique using a machine learning algorithm was proposed. The proposed algorithm can be broken down into two steps: artery wall region detection in M-mode ultrasound signal and artery lumen diameter estimation by tracking the artery wall using a correlation-based technique. A machine learning-based artery region classifier (detector), was developed to check if the ultrasound transducer (UT) is over the artery, during signal acquisition. In order to check if the UT is over the artery, a machine learning-based classifier is trained, evaluated, and tested to detect for the region containing the artery wall in the M-mode image formed by the UT placed in the neck region ( $2 \mathrm{~cm}$ above the carotid bulb). A ground truth about the location of a region containing the artery wall region should be known to train, evaluate, and test the performance of the machine learning-based artery region classifier (detector). A manual segmentation approach as explained in Section 4.1.1, was used to create the ground truth about the region with and without artery walls. The region (a subset of Mmode image) labeled during the ground truth creation process was called segmented windows. Fast and slow-time features of each segmented window, as explained in Section 4.1.2, were extracted and used as an input to machine learning-based classifiers. Several machine learning-based classifiers (LDA, KNN, RF, XGB, and $\mathrm{CNN}$ ) was selected, and 
their performance based on the features extracted were compared in two experimental environments of artery phantoms constructed and human in-vivo subjects.

In the phantom experiments, two experimental environments were created to simulate the artery and its motion during the cardiac cycle using two different phantoms, named Phantom A and Phantom B. The Phantom A simulated a scattering noise-free environment by simulating the artery movement using a polymer tube immersed in water. The change in diameter of the artery due to the cardiac cycle was simulated by periodically pumping water into the polymer tube using a syringe. This noiseless (scattering noise) environment was used to evaluate and compare the performance of the proposed algorithms. As discussed in Section 5.1.1, KNN performed better than other algorithms with an accuracy of $99.7 \%$. CNN classifier, which automatically extracted the features from the segmented windows, performed the worst with an accuracy of $70.3 \%$. It is because the $\mathrm{CNN}$, which automatically extracts features from the M-mode image, fails to differentiate between proximal and distal walls, while the classifiers that are based on the features extracted manually were able to distinguish between a wall and no boundary region. Since the Phantom A provided an ideal environment (scattering noise-free environment), the classifiers were then tested on the ultrasound rf signal acquired from the Phantom B. In the Phantom B, the polymer tube simulating the artery was surrounded with tissue-mimicking hydrogel-based material. It simulates a more realistic human in-vivo environment. Carbon particles were dispersed inside the hydrogel- material, surrounding the polymer tube in the Phantom B, in order to replicate the ultrasound scattering occurring inside a human in-vivo 
tissue. Such carbon particles produce scattering echoes (speckle noises) in the acquired ultrasound rf signal.

As explained in Section 5.1.2, CNN performed better than other algorithms with an accuracy of $97 \%$. CNN classifier, which automatically extracted the features from the segmented windows, performed better than the algorithms that were based on the features that were manually extracted in the case of the Phantom B. This showed that the features that were automatically obtained by the $\mathrm{CNN}$ were better than the features that were manually extracted. The Random Forest (RF) algorithm performed the worst in the case of the Phantom B experiments with an accuracy of $75.4 \%$. In the case of human in-vivo experiments, the data were obtained from 8 human subjects after obtaining permission from the Carleton ethics board. Among the human in-vivo data collected at the common carotid artery, six were used for training and evaluating the classifiers, while two were used to test the classifiers. The results of the classifiers for human in-vivo experiments are explained in Section 5.1.3. Similar to the results of classifiers in the Phantom B experiment (hydrogelbased phantom with scatterers), CNN performs better than the other algorithms with an accuracy of $88.9 \%$ accuracy. The KNN algorithm performs the worst with an accuracy of $79.6 \%$ in the case of human in-vivo experiments. The results of the algorithm show that CNN was able to extract features that are better than the features that are manually extracted. It was also able to generalize to unseen data from a different person better than the rest of the algorithms considered in this thesis. A detailed comparison and the performance analysis of the algorithms considered were explained in Section 5.1.3. The performance of classifiers on the phantom based and human in-vivo experiments described 
above were on the ultrasound data acquired from the PICUS clinical ultrasound acquisition system. On assessing the performance of classifiers on the data obtained from PICUS clinical ultrasound probe, it was observed that CNN performed better (explained in terms of F1-score and accuracy in Section 5.1.1-5.1.3) than the other classifiers in case of the Phantom B and human in-vivo experiments. Hence, $\mathrm{CNN}$ was trained, evaluated and tested on the human in-vivo data obtained from the WUS system.

The performance of CNN on the data acquired from the WUS system is explained in Section 5.1.4. CNN was able to detect the segmented window containing proximal and distal artery walls at the common carotid artery of the human in-vivo subjects with an accuracy of $88.3 \%$, which is very similar to the results obtained in human in-vivo experiments from the PICUS clinical ultrasound acquisition system. The reason behind the misclassification of CNN in both the human in-vivo data obtained from the PICUS clinical ultrasound system and WUS system is discussed in detail in Section 5.1.4. Thus, an automatic artery wall region classifier has been proposed and tested on data acquired from two different ultrasound acquisition system. Once the region in which the artery wall to be tracked is identified with CNN based artery wall region classifier, the change in lumen diameter of the artery is estimated using the peak detection technique, and the correlationbased artery wall tracking method explained in Section 4.2. The artery wall detection and lumen diameter estimation technique were used to estimate the inner diameter of the polymer tube used in the phantom experiments. The results of the diameter estimation technique on phantom-based experiments are explained in Section 5.2.1 and 5.2.2. The inner diameter of the moving polymer tube is obtained using the artery diameter estimation 
technique, and there is no ground truth to compare the actual value of the diameter change due to the manual pumping. Since the tube was manually pumped with water to simulate the cardiac cycle of the artery, the amount of water pumped into the tube (causing the expansion and contraction of the tube) was difficult to control or measure. The inner diameter of the tube in the Phantom A and B at rest was measured by a caliper having a measurement resolution of $0.01 \mathrm{~mm}$. In case of Phantom A, it was $9.48 \pm 0.04 \mathrm{~mm}$, while the minimum inner diameter of the polymer tube during the induced motion (as explained in Section 5.2.1) was measured to be $9.437 \pm 0.028 \mathrm{~mm}$.

The difference in the actual diameter and the average of the minimum inner diameter of the polymer tube measured using the artery diameter estimation technique is due to both experimental error and the error in the measurement technique. Similarly, for Phantom B, the inner diameter of the polymer tube was measured to be $9.55 \pm 0.05 \mathrm{~mm}$ at rest. The minimum inner diameter of the polymer tube measured using artery diameter estimation technique (as explained in Section 5.2.2) during the induced motion was $9.599 \pm 0.030$ $\mathrm{mm}$. The difference in the actual diameter and the average of the minimum inner diameter of the polymer tube measured using the artery diameter estimation technique is due to both experimental error and the error in the measurement technique. The artery wall detection and lumen diameter estimation method is used to measure the systolic and diastolic diameter of the carotid artery in human in-vivo experiments, and the results are explained in Section 5.2.3 and 5.2.4. The artery lumen diameter of a human subject was measured from the data acquired using both the PICUS clinical ultrasound system and the WUS system. The systolic and diastolic diameter of the artery was measured to be $5.938 \pm 0.099$ 
$\mathrm{mm}$ and $5.379 \pm 0.069 \mathrm{~mm}$ for data from the PICUS clinical ultrasound system, while the systolic and diastolic diameter estimated from the WUS system data was $5.898 \pm 0.019$ $\mathrm{mm}$ and $5.289 \pm 0.021 \mathrm{~mm}$. The difference in the systolic and diastolic diameter measurement between two acquisition system was probably due to: the change in the diameter of the artery due to the pressure applied by the ultrasound transducers; measurement error due to the artery diameter estimation technique; motion artefact due to the movement of the human subject during the data acquisition and change in the blood pressure of the human subject (causes the change in systolic and diastolic lumen diameter). Though the results obtained from the proposed algorithm on PICUS clinical ultrasound system and the WUS system are only $0.090 \mathrm{~mm}$ and $0.040 \mathrm{~mm}$ different from each other, the difference or the error between the actual value of the lumen diameter change and the measure diameter was not measured.

The existing semi-automatic, and thresholding-based algorithms to detect and estimate artery diameter is evaluated based on the custom evaluation metric based on the threshold value set. Hence, a direct comparison in performance between the proposed algorithm and the existing algorithm could not be made. Based on the results discussed in Chapter 5, it is seen that the $\mathrm{CNN}$ for the artery detection and autocorrelation-based tracking technique for the lumen diameter change can be used to measure the arterial distension with the help of A-mode transducer. The automatic artery wall detection technique eliminates the need for expensive imaging hardware and technique, while the autocorrelation-based tracking technique is relied upon to estimate the change in the lumen diameter. The estimated change in the systolic and the diastolic diameter can be used in conjunction with the 
pressure difference measured using one of the techniques discussed in Section 2.2.5, to estimate the stiffness of the artery. The proposed technique could be useful in developing an artery health monitoring system using ultrasound.

\subsection{Future Research}

As mentioned before, the primary intent of this research was developing an artery detection and lumen diameter tracking algorithm to estimate the change in the systolic and diastolic lumen diameter using a low-cost WUS for continuous monitoring of arterial properties. The research was conducted to develop an automatic artery detection and lumen diameter tracking algorithm to measure the systolic and diastolic diameter in the M-mode ultrasound rf signal. The following section outlines some suggestions for future work and study.

- The ultrasound data acquisition systems used in this thesis acquired the ultrasound rf signal and stored it, and then post-processed the stored signal to locate and track the artery lumen diameter. A new acquisition setup and procedure using the WUS system could be developed to allow real-time processing of the acquired signal to detect artery wall region and track the artery lumen diameter. The new setup could also provide real-time feedback to the operator trying to locate the carotid artery.

- In the proposed algorithm, once the artery region detector classifies the segmented region with a given level of accuracy, the region classified as the proximal and the distal wall region is not checked again for verification. More studies and experiments to evaluate the advantages of verifying the artery wall region should be carried out. 
- The ultrasound rf signal acquired with the WUS during the experiments on human in-vivo contained some high amount of scattering and/or electric noises. Processing signals in the frequency domain may help to find the source and the characteristics of the noises. A proper digital filter can be designed to remove noises and improve the SNR of the desired ultrasonic signal from the artery walls of interest. Further, more frequency domain and auto-correlation based features could be investigated as an input to the machine learning classifiers.

- Since the artery and the vein have a similar characteristic of expansion and contraction due to the cardiac cycle, the current algorithm proposed, use the pattern that is observed using the cardiac cycle to differentiate the moving tissues with the stationary tissues. It does not look at the pattern of the artery or vein for the segregation. Thus, the algorithm could detect the jugular vein instead of an artery. More studies should be done to modify the algorithm only to detect artery and exclude the jugular vein. The ECG signals could be used in conjunction to separate the artery with vein based on the cardiac cycle. Further, the ECG sensor in conjugation with the ultrasound acquisition system could be used to verify the pattern of the diameter change tracked using the proposed algorithm.

- A statistical study involving more human subjects to measure and compare the performance of CNN on data from PICUS clinical ultrasound imaging system and WUS system should be carried out.

- A gold standard measurement technique such as laser displacement sensor can be used to track the change in diameter of the polymer tube in water (Phantom A) and evaluate the performance of the proposed algorithms for artery detection and lumen 
diameter measurement using ultrasound. This laser measurement can be used to evaluate the error of the proposed algorithm on the data acquired using the ultrasound acquisition systems.

- The artery wall lumen diameter can be manually measured by a certified physician or a medical sonographer and can be used as a gold standard to estimate the error in the artery lumen diameter tracking algorithm.

- It is recommended to optimize the algorithm to perform clinical trials in conjunction with a suitable blood pressure measurement technique to investigate the ability of the measurement system to estimate the change in stiffness between a healthy individual and a person with coronary heart disease.

- A statistical study can be conducted to measure the change in diameter of the brachial artery and its potential use in flow-mediated dilatation (FMD) test. FMD is used to measure the endothelial function in the brachial artery [56], [57].

- The inter and intra operator variability in the performance of the proposed algorithm can be evaluated by estimating the diameter of the artery from multiple acquisitions of the same human test subject.

- A statistical study can be conducted to measure the change in diameter of the jugular vein and its potential use in jugular vein distension measurement. Jugular vein distension waveform is used in assessing the severity and response to the treatment of congestive heart failure [96].

- The committee of classifiers might be able to perform better results than the individual classifiers, but the time taken for each of the classifiers to make a decision could be way higher than selecting the results of an individual classifier. 
Hence, more investigation could be carried out to analyze the trade-off between the time and performance of the classifiers. 


\section{Reference}

[1] V. S. Benjamin EJ, Muntner P, Alonso A, Bittencourt MS, Callaway CW, Carson AP, Chamberlain AM, Chang AR, Cheng S, Das SR, Delling FN, Djousse L, Elkind MSV, Ferguson JF, Fornage M, Jordan LC, Khan SS, Kissela BM, Knutson KL, Kwan TW, Lackland DT, Lewis TT, Lic, "Heart Disease and Stroke Statistics-2019 At-a-Glance Heart Disease, Stroke and other Cardiovascular Diseases," Am. Hear. Assoc., pp. 1-5, 2019.

[2] E. J. Benjamin et al., "Heart Disease and Stroke Statistics," Circulation, vol. 117, no. $4,2018$.

[3] F. Sanchis-Gomar, C. Perez-Quilis, R. Leischik, and A. Lucia, "Epidemiology of coronary heart disease and acute coronary syndrome," Ann. Transl. Med., vol. 4, no. 13, pp. 1-12, 2016.

[4] A. Go et al., "Heart Disease and Stroke Statistics-2008 Update," Circulation, vol. 117, no. 4, 2007.

[5] G. Filice et al., "Arterial Stiffness Evaluation in HIV-Positive Patients: A Multicenter Matched Control Study," Am. J. Roentgenol., vol. 197, no. 5, pp. $1258-$ 1262, 2011.

[6] N. Leone et al., "Distension of the carotid artery and risk of coronary events: The three-city study," Arterioscler. Thromb. Vasc. Biol., vol. 28, no. 7, pp. 1392-1397, 2008.

[7] J. M. Dijk, A. Algra, Y. Van Der Graaf, D. E. Grobbee, and M. L. Bots, "Carotid stiffness and the risk of new vascular events in patients with manifest cardiovascular disease. The SMART study,” Eur. Heart J., vol. 26, no. 12, pp. 1213-1220, 2005. 
[8] Y. Myung et al., "The correlation of carotid artery stiffness with heart function in hypertensive patients," J. Cardiovasc. Ultrasound, vol. 20, no. 3, pp. 134-139, 2012.

[9] C. Yuan, J. Wang, and M. Ying, "Predictive value of carotid distensibility coefficient for cardiovascular diseases and all-cause mortality: A meta-analysis,” PLoS One, vol. 11, no. 4, pp. 1-15, 2016.

[10] S. Laurent et al., "Expert consensus document on arterial stiffness: methodological issues and clinical applications.," Eur. Heart J., vol. 27, no. 21, pp. 2588-2605, Nov. 2006.

[11] J. Angus et al., "'Sneaky disease': the body and health knowledge for people at risk for coronary heart disease in Ontario, Canada.," Soc. Sci. Med., vol. 60, no. 9, pp. 2117-2128, May 2005.

[12] J. Angus et al., ““" Sneaky disease "”: the body and health knowledge for people at risk for coronary heart disease in Ontario , Canada," vol. 60, pp. 2117-2128, 2005.

[13] T. S. Schoot, M. Weenk, T. H. van de Belt, L. J. L. P. G. Engelen, H. van Goor, and S. J. H. Bredie, “A New Cuffless Device for Measuring Blood Pressure: A RealLife Validation Study.," J. Med. Internet Res., vol. 18, no. 5, p. e85, May 2016.

[14] R. T. Worthing, "Using Ultrasound to Measure Arterial Diameter for the Development of Wearable Blood Pressure Monitoring Device.," M.Asc Thesis, University of British Columbia, 2014.

[15] A. Chandrasekhar, C.-S. Kim, M. Naji, K. Natarajan, J.-O. Hahn, and R. Mukkamala, "Smartphone-based blood pressure monitoring via the oscillometric finger-pressing method," Sci. Transl. Med., vol. 10, no. 431, 2018. 
[16] H. Luo et al., "Smartphone-Based Blood Pressure Measurement Using Transdermal Optical Imaging Technology," Circ. Cardiovasc. Imaging, vol. 12, no. 8, Aug. 2019.

[17] R. Mukkamala, "Blood Pressure With a Click of a Camera?," Circ. Cardiovasc. Imaging, vol. 12, no. 8, Aug. 2019.

[18] M. A. Gutierrez, P. E. Pilon, S. G. Lage, L. Kopel, R. T. Carvalho, and S. S. Furuie, "Automatic measurement of carotid diameter and wall thickness in ultrasound images," pp. 359-362, 2003.

[19] B. E. Hunt, D. C. Flavin, E. Bauschatz, and H. M. Whitney, "Accuracy and robustness of a simple algorithm to measure vessel diameter from B-mode ultrasound images," J. Appl. Physiol., vol. 120, no. 11, pp. 1374-1379, 2016.

[20] A. K. Sahani, J. Joseph, and M. Sivaprakasam, "Automatic measurement of lumen diameter of carotid artery in A-Mode ultrasound," Proc. Annu. Int. Conf. IEEE Eng. Med. Biol. Soc. EMBS, pp. 3873-3876, 2013.

[21] A. K. Sahani, M. I. Shah, J. Joseph, and M. Sivaprakasam, "Carotid and Jugular Classification in ARTSENS," IEEE J. Biomed. Heal. Informatics, vol. 20, no. 2, pp. 440-449, 2016.

[22] J. Joseph and V. Jayashankar, "A Virtual Instrument for Automated Measurement of Arterial Compliance,” J. Med. Device., vol. 4, no. 4, p. 045004, 2010.

[23] M. Naghavi, A. A. Yen, A. W. H. Lin, H. Tanaka, and S. Kleis, "New Indices of Endothelial Function Measured by Digital Thermal Monitoring of Vascular Reactivity: Data from 6084 Patients Registry," Int. J. Vasc. Med., vol. 2016, no. 4, pp. 1-8, 2016.

[24] I. Song et al., "Design and implementation of a New Wireless carotid neckband 
doppler system with wearable ultrasound sensors: Preliminary results," Appl. Sci., vol. 9 , no. $11,2019$.

[25] C. Wang et al., "Monitoring of the central blood pressure waveform via a conformal ultrasonic device," Nat. Biomed. Eng., vol. 2, no. 9, pp. 687-695, 2018.

[26] A. Huang, M. Yoshida, Y. Ono, and S. Rajan, "Continuous measurement of arterial diameter using wearable and flexible ultrasonic sensor," in 2017 IEEE International Ultrasonics Symposium (IUS), 2017, pp. 1-4. [24] G. K. Lewis, M. D. Langer, C. R. Henderson, and R. Ortiz, "Design and evaluation of a wearable self-applied therapeutic ultrasound device for chronic myofascial pain," Ultrasound Med. Biol., vol. 39, no. 8, pp. 1429-1439, 2013.

[27] G. K. Lewis, M. D. Langer, C. R. Henderson, and R. Ortiz, "Design and evaluation of a wearable self-applied therapeutic ultrasound device for chronic myofascial pain," Ultrasound Med. Biol., vol. 39, no. 8, pp. 1429-1439, 2013.

[28] I. AlMohimeed and Y. Ono, "Flexible and Wearable Ultrasonic Sensor for Assessment of Skeletal Muscle Contractile Properties," in 2019 IEEE International Conference on Flexible and Printable Sensors and Systems (FLEPS), 2019, pp. 13.

[29] Jerry.1.prince and J. M. Links, Medical imaging signals and systems, 2nd ed. Pearson Prentice Hall, 2013, pp. 200-356..

[30] A. Tharwat, T. Gaber, A. Ibrahim, and A. E. Hassanien, "Linear discriminant analysis: A detailed tutorial," AI Commun., vol. 30, no. 2, pp. 169-190, 2017.

[31] C. Andersson, "Multivariate signal approaches for object detection using microwave sensing,” 2013. 
[32] F. Pedregosa et al., "Scikit-learn: Machine Learning in Python," vol. 12, pp. 28252830, 2012.

[33] S. Shalev-Shwartz and S. Ben-David, Understanding machine learning: From theory to algorithms, vol. 9781107057.2013.

[34] T. Chen and C. Guestrin, "XGBoost: A scalable tree boosting system," Proc. ACM SIGKDD Int. Conf. Knowl. Discov. Data Min., vol. 13-17-Augu, pp. 785-794, 2016.

[35] T. T. Hastie, R. Tibshirani, J. Friedman, The Elements of Statistical Learning: Data Mining, Inference, and Prediction, vol. 26, no. 4. 2009.

[36] S. Esmeir and S. Markovitch, "Anytime learning of decision trees," J. Mach. Learn. Res., vol. 8, pp. 891-933, 2007.

[37] B. Gupta, A. Rawat, A. Jain, A. Arora, and N. Dhami, "Analysis of Various Decision Tree Algorithms for Classification in Data Mining," Int. J. Comput. Appl., vol. 163, no. 8, pp. 15-19, 2017.

[38] Y. Wang, "Comparing Linear Discriminant Analysis with Classification Trees Using Forest Landowner Survey Data as a Case Study with Considerations for Optimal Biorefinery Siting,” 2008.

[39] T. Chen and C. Guestrin, "XGBoost: A scalable tree boosting system," Proc. ACM SIGKDD Int. Conf. Knowl. Discov. Data Min., vol. 13-17-Augu, pp. 785-794, 2016.

[40] J. Kiefer and J. Wolfowitz, "Stochastic Estimation of the Maximum of a Regression Function," Ann. Math. Stat., vol. 23, no. 3, pp. 462-466, 1952.

[41] J. H. Friedman, "Greedy Function Approximation: A Gradient Boosting Machine," Ann. Stat., vol. 29, no. 2, pp. 1189-1232, 1999. 
[42] É. D. Fabian Pedregosa, Gaël Varoquaux, Alexandre Gramfort, Vincent Michel, Bertrand Thirion, Olivier Grisel, Mathieu Blondel, Peter Prettenhofer, Ron Weiss, Vincent Dubourg, Jake Vanderplas, Alexandre Passos, David Cournapeau, Matthieu Brucher, Matthieu Perrot, "Scikit-learn: Machine Learning in Python," J. Mach. Learn. Res., p. 2825-2830, 2011.

[43] Y. Lecun, L. Bottou, Y. Bengio, and P. Ha, "LeNet," Proc. IEEE, no. November, pp. 1-46, 1998.

[44] A. Krizhevsky, I. Sutskever, and G. E. Hinton, "ImageNet Classification with Deep Convolutional Neural Networks," Handb. Approx. Algorithms Metaheuristics, pp. 60-1-60-16, 2007.

[45] J. Duchi, E. Hazan, and Y. Singer, "Adaptive Subgradient Methods for Online Learning and Stochastic Optimization,” Jmlr, pp. 1-40, 2011.

[46] T. Khamdaeng, J. Luo, J. Vappou, P. Terdtoon, and E. E. Konofagou, “Arterial stiffness identification of the human carotid artery using the stress-strain relationship in vivo," Ultrasonics, vol. 52, no. 3, pp. 402-411, 2012.

[47] P. J. Czuwala, "McDonald's Blood flow in arteries: Theoretical, experimental and clinical principles," Echocardiography. Vasc. Biol., vol. 8, no. 3, pp. 405-406, 2007..

[48] S. Laurent et al., "Elastic modulus of the radial artery wall material is not increased in patients with essential hypertension," Arterioscler. Thromb. Vasc. Biol., vol. 14, no. 7, pp. 1223-1231, 1994.

[49] A. Manbachi, Y. Hoi, B. A. Wasserman, E. G. Lakatta, and D. A. Steinman, "On the shape of the common carotid artery with implications for blood velocity 
profiles," Physiol. Meas., vol. 32, no. 12, pp. 1885-1897, Oct. 2011.

[50] Y. R. Limbu, G. Gurung, R. Malla, R. Rajbhandari, and S. R. Regmi, "Assessment of carotid artery dimensions by ultrasound in non-smoker healthy adults of both sexes.," Nepal Med. Coll. J., vol. 8, no. 3, pp. 200-3, 2006.

[51] J. Krejza et al., "Carotid artery diameter in men and women and the relation to body and neck size," Stroke, vol. 37, no. 4, pp. 1103-1105, 2006.

[52] F. Article, "Medical gallery of Blausen Medical 2014," WikiJournal Med., vol. 1, no. $2,2014$.

[53] G. Parati, G. Magnaghi, A. Giglio, L. Lonati, G. Bilo, and G. Leonetti, "Association Between Arterial Stiffness and Carotid Atherosclerosis," High Blood Press. Cardiovasc. Prev., vol. 12, no. 3, p. 154, 2006.

[54] B. Jóźwiak, M. Orczykowska, and M. Dziubiński, "Fractional Generalizations of Maxwell and Kelvin-Voigt Models for Biopolymer Characterization," PLoS One, vol. 10, no. 11, pp. 1-19, 2015.

[55] D. H. Bergel, "The static elastic properties of the arterial wall," J. Physiol., vol. 156, no. 3, pp. 445-457, May 1961.

[56] O. T. Raitakari and D. S. Celermajer, "Flow-mediated dilatation," Br. J. Clin. Pharmacol., vol. 50, no. 5, pp. 397-404, 2000.

[57] T. Maruhashi et al., "Relationship between flow-mediated vasodilation and cardiovascular risk factors in a large community-based study," Heart, vol. 99, no. 24, pp. 1837-1842, 2013.

[58] R. A. Harris, S. K. Nishiyama, D. W. Wray, and R. S. Richardson, "Ultrasound assessment of flow-mediated dilation," Hypertens. (Dallas, Tex. 1979), vol. 55, no. 
5, pp. 1075-1085, May 2010.

[59] M. A. Said, R. N. Eppinga, E. Lipsic, N. Verweij, and P. van der Harst, "Relationship of Arterial Stiffness Index and Pulse Pressure With Cardiovascular Disease and Mortality," J. Am. Heart Assoc., vol. 7, no. 2, p. e007621, 2018.

[60] J. Y. Cho and K. H. Kim, "Evaluation of Arterial Stiffness by Echocardiography: Methodological Aspects," Chonnam Med. J., vol. 52, no. 2, pp. 101-106, May 2016.

[61] E. C. Godia et al., "Carotid artery distensibility: A reliability study," J. Ultrasound Med., vol. 26, no. 9, pp. 1157-1165, 2007.

[62] P. Tozzi, A. Corno, and D. Hayoz, "Definition of arterial compliance," Am. J. Physiol. Circ. Physiol., vol. 278, no. 4, pp. H1407-H1407, 2000.

[63] J. Booth, "A Short History of Blood Pressure Measurement," J. R. Soc. Med., vol. 70, no. 11, pp. 793-799, 1977.

[64] A. Benmira et al., "From Korotkoff and Marey to automatic non-invasive oscillometric blood pressure measurement: does easiness come with reliability?," Expert Rev. Med. Devices, vol. 13, no. 2, pp. 179-189, 2016.

[65] G. M. Drzewiecki, J. Melbin, and A. Noordergraaf, "Arterial tonometry: Review and analysis," J. Biomech., vol. 16, no. 2, pp. 141-152, 1983.

[66] R. Maggi, V. Viscardi, T. Furukawa, and M. Brignole, "Non-invasive continuous blood pressure monitoring of tachycardic episodes during interventional electrophysiology," Europace, vol. 12, no. 11, pp. 1616-1622, 2010.

[67] R. S. Reneman, T. van Merode, P. J. Brands, and A. P. Hoeks, "Inhomogeneities in arterial wall properties under normal and pathological conditions.," J. Hypertens. Suppl., vol. 10, no. 6, pp. S35-9, Aug. 1992. 
[68] O. A. Olowoyeye, "Flow mediated dilatation ( FMD ) assessment via an MRI integrated signal intensity ( IntSI ) approach : A comparison with ultrasound,”, Ph.D dissertation, Institute of Medical Science University of Toronto, Toronto, 2017.

[69] D. S. Celermajer et al., "Non-invasive detection of endothelial dysfunction in children and adults at risk of atherosclerosis," Lancet, vol. 340, no. 8828, pp. 1111$1115,1992$.

[70] A. K. Sahani, J. Joseph, and M. Sivaprakasam, “Automated system for imageless evaluation of arterial compliance," Proc. Annu. Int. Conf. IEEE Eng. Med. Biol. Soc. EMBS, pp. 227-231, 2012.

[71] Z. Wang et al., "A flexible ultrasound transducer array with micro-machined bulk pzt," Sensors (Switzerland), vol. 15, no. 2, pp. 2538-2547, 2015.

[72] N. Dimitrova et al., "Public Access NIH Public Access," PLoS One, vol. 32, no. 7, pp. 736-740, 2017.

[73] Ibrahim and AlMohimeed, "Development of Wearable Ultrasonic Sensor s for Monitoring Muscle Contraction," M.Asc. thesis, Ottawa-Carleton Institute for Biomedical Engineering, Carleton Univ., Ottawa, Aug.2013.

[74] C. L. de Korte, H. H. G. Hansen, and A. F. W. van der Steen, "Vascular ultrasound for atherosclerosis imaging," Interface Focus, vol. 1, no. 4, pp. 565-575, 2011.

[75] J. Lang, "Ultrasound Artefacts," Diagnostic Ultrasound Small Anim. Pract., no. April, pp. 20-25, 2007.

[76] Z. Qu and B. Eng, Development of Ultrasound Measurement System for Characterizing Viscoelastic Materials Using Ultrasound Reflectance Method, no. September. 2010. 
[77] M. Earle, G. De Portu, and E. Devos, "Agar ultrasound phantoms for low-cost training without refrigeration," African J. Emerg. Med., vol. 6, no. 1, pp. 18-23, 2016.

[78] M. Doctor, P. Olivieri, S. D. Siadecki, G. Rose, A. Drake, and T. Saul, "Sonographer preference of ballistic gelatin concentration used to create DVT training phantoms," Am. J. Emerg. Med., vol. 35, no. 1, pp. 178-179, 2017.

[79] D. A. Kenwright, N. Laverick, T. Anderson, C. M. Moran, and P. R. Hoskins, "Wallless flow phantom for high-frequency ultrasound applications," Ultrasound Med. Biol., vol. 41, no. 3, pp. 890-897, 2015.

[80] S. L. Chao, K. C. Chen, L. W. Lin, T. L. Wang, and C. F. Chong, "Ultrasound phantoms made of gelatin covered with hydrocolloid skin dressing," J. Emerg. Med., vol. 45, no. 2, pp. 240-243, 2013.

[81] R. O. Bude and R. S. Adler, "An easily made, low-cost, tissue-like ultrasound phantom material," J. Clin. Ultrasound, vol. 23, no. 4, pp. 271-273, 1995.

[82] E. Jones, T. Oliphant, P. Peterson, and others, "SciPy: Open source scientific tools for Python." [Online]. Available: http://www.scipy.org/.

[83] V. Raj Kiran, J. Joseph, M. I. Shah, and M. Sivaprakasam, "An image-free ultrasound method to estimate artery wall thickness surrogate for screening," 2017 IEEE Int. Symp. Med. Meas. Appl. MeMeA 2017 - Proc., pp. 326-331, 2017.

[84] P. Mousavi, P. A. Isotalo, M. Moradi, D. R. Siemens, E. E. Sauerbrei, and P. Abolmaesumi, "A new approach to analysis of RF ultrasound echo signals for tissue characterization: animal studies," Med. Imaging 2007 Ultrason. Imaging Signal Process., vol. 6513, p. 65130P, 2007. 
[85] J. A. Fiz et al., "Fractal dimension analysis of malignant and benign endobronchial ultrasound nodes,” BMC Med. Imaging, vol. 14, no. 1, pp. 4-9, 2014.

[86] P. Nayyeri, "Analyzing Electrocardiography (ECG) Signal using Fractal Method," vol. 7, no. 2, pp. 498-505, 2017.

[87] G. Tremberger, R. Sullivan, S. Dehipawala, and P. Schneider, "Fractal Dimension Analysis of EEG Time Series Signal Buried in Random Noise," Eeecos.Org, pp. 590-593, 2016.

[88] B. KumarSingh, K. Verma, and A. S. Thoke, "Investigations on Impact of Feature Normalization Techniques on Classifier\&apos;s Performance in Breast Tumor Classification," Int. J. Comput. Appl., vol. 116, no. 19, pp. 11-15, 2015.

[89] N. Chawla, K. Bowyer, L. O. Hall, and W. Philip Kegelmeyer, SMOTE: Synthetic Minority Over-sampling Technique, vol. 16. 2002.

[90] J. Bergstra and Y. Bengio, "Random Search for Hyper-parameter Optimization," J. Mach. Learn. Res., vol. 13, pp. 281-305, Feb. 2012.

[91] Q. Guo, "Minowski measure of asymmetry," Br. J. Psychiatry, vol. 111, no. 479, pp. 1009-1010, 1965.

[92] F. Pedregosa et al., "Scikit-learn: Machine Learning in $\{\mathrm{P}\}$ ython," J. Mach. Learn. Res., vol. 12, pp. 2825-2830, 2011.

[93] Z. Zhang and M. R. Sabuncu, "Generalized Cross Entropy Loss for Training Deep Neural Networks with Noisy Labels,” no. NeurIPS, 2018.

[94] S. van der Walt et al., "scikit-image: image processing in Python," PeerJ, vol. 2, p. p 453, 2014.

[95] K. D. Lloyd, E. Barinas-Mitchell, L. H. Kuller, R. H. MacKey, E. A. Wong, and K. 
Sutton-Tyrrell, "Common carotid artery diameter and cardiovascular risk factors in overweight or obese postmenopausal women," Int. J. Vasc. Med., vol. 2012, 2012.

[96] J. M. S. Chua Chiaco, N. I. Parikh, and D. J. Fergusson, "The jugular venous pressure revisited," Cleve. Clin. J. Med., vol. 80, no. 10, pp. 638-644, 2013.

[97] T. Higuchi, "Approach to an irregular time series on the basis of the fractal theory," Phys. D Nonlinear Phenom., 1988. 


\section{Appendix}

\section{Fractal Dimension}

The Higuchi transform algorithm [97] is used to calculate the fractal dimension. It decomposes the signal into different scales and evaluates the signal complexity. Higuchi's algorithm is proven to be a stable and accurate method to estimate the fractal dimension of time series and works well even for time series with very few samples [84], [87], [97].

The algorithm computes the mean length of the signal at different scales, plots a log-log graph of length versus scale, and measures the slope of the linear fit of this graph as the fractal dimension. The segmented window is a matrix with its columns representing depth (fast-time) and rows representing the frames (slow-time). Thus, the segmented window can be considered as the subset of the ultrasound rf signal while the probe is in a fixed position over time. Each sample of the ultrasound rf signal in the segmented window over time (slow-time) forms a time series $\{X(1), X(2), \ldots, X(N)\} . \mathrm{N}$ is given as the height of the segmented window. From this time series, we first construct $\mathrm{k}$ new time series of the form provided in Equation (1).

$$
X_{k}^{m}:\left\{X(m), X(m+k), X(m+2 k) \ldots, X\left(+\left[\frac{N-m}{k}\right] . k\right)\right\}
$$

Where $\mathrm{k}$ is the sampling time interval (which determines the scale, $k<N$ ) and $m=$ $1,2, \ldots k-1$. Both $m$ and $k$ are integers. The length of each time series, $L_{m}(k)$, is defined in the Equation (2). 


$$
\begin{aligned}
L_{m}(k)=1 / k & \times\left(\frac{N-1}{\left[\frac{N-m}{k}\right] \cdot k}\right) \times \sum_{i=1}^{\left[\frac{N-m}{k}\right]} \mid X(m+i k) \\
& -X(+(-1) \cdot k) \mid
\end{aligned}
$$

The average value of $L_{m}(k)$ over $k$ sets, $L(k)$, is the so-called length of the time series at scale $k$. This procedure is repeated for each $k$ ranging from 1 to $k_{\max }$. A line is fitted to values of $\ln \mathrm{L}(k)$ versus $\ln (1 / k)$ and the slope of this line is considered as the fractal Dimension. The number of the samples, $N$, and the nature of the time series determine the optimal value of the parameter $k_{\max }$. The value of $k_{\max }$ in this case, it is experimentally selected to be 15 for the time series of length 60 .

\section{Performance of machine learning algorithm in Phantom A}

\section{LDA}
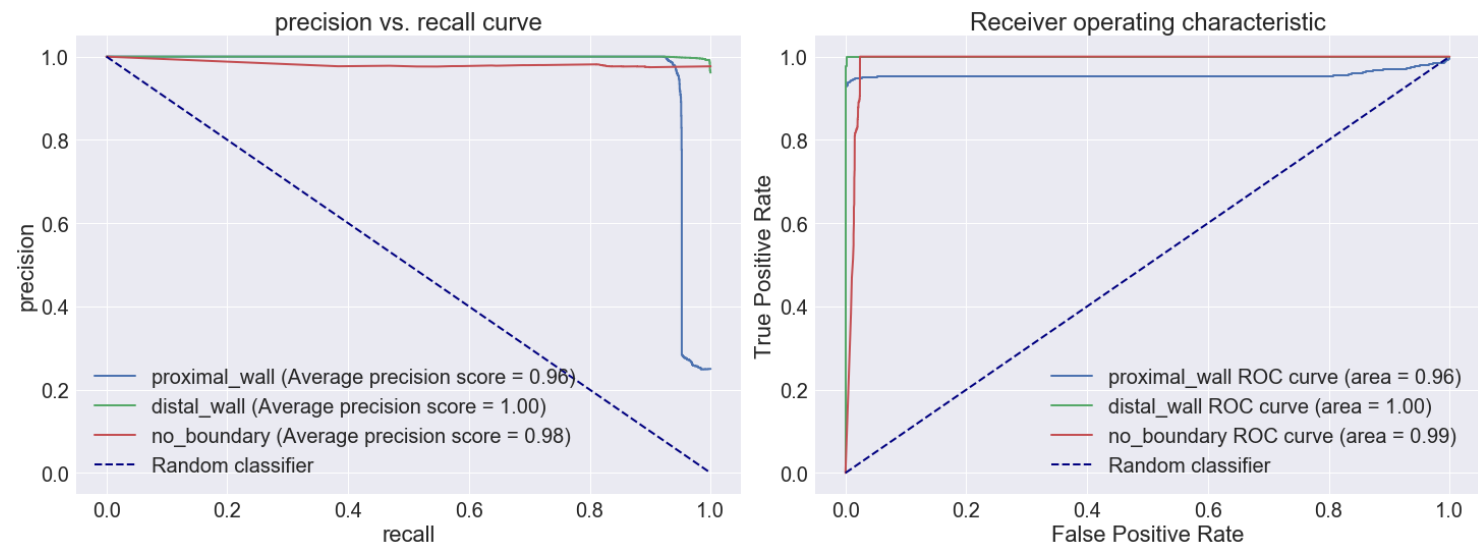

\section{KNN}



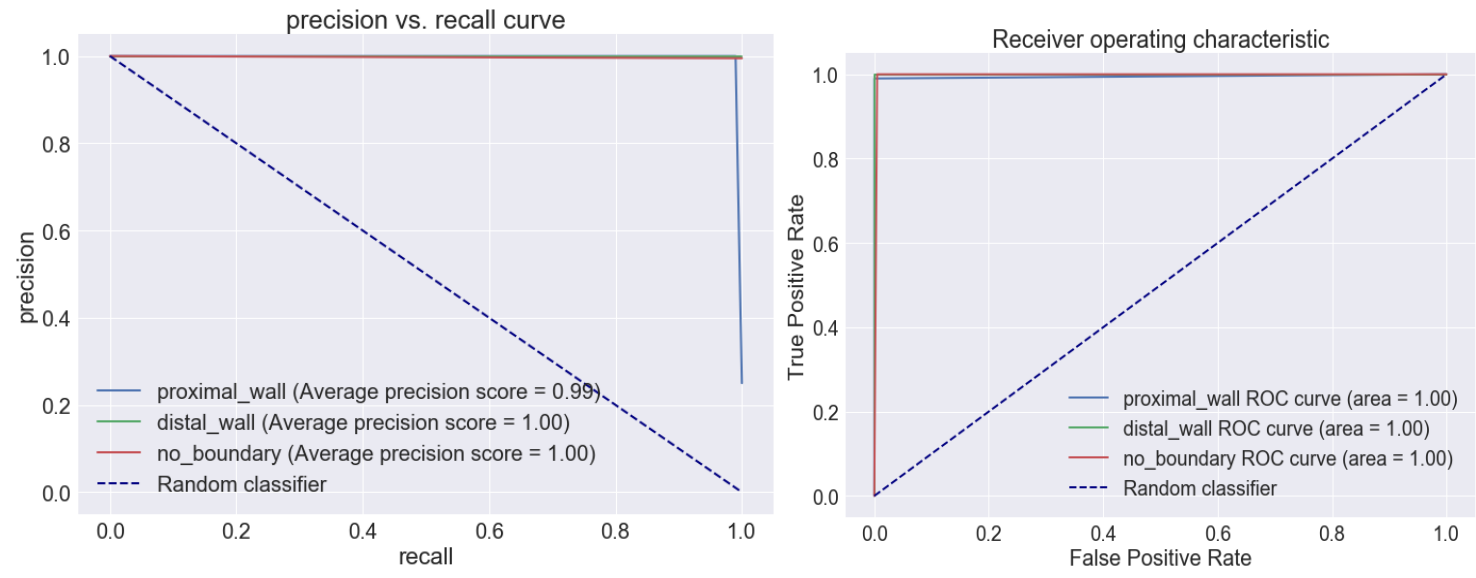

RF
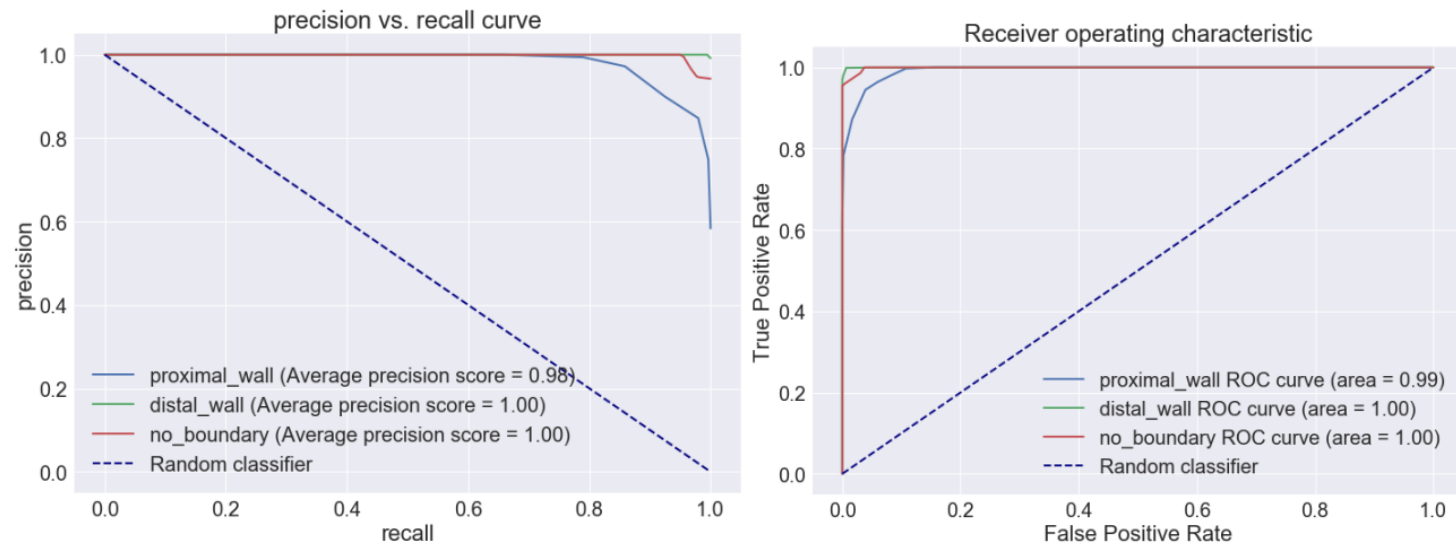

\section{XGB}



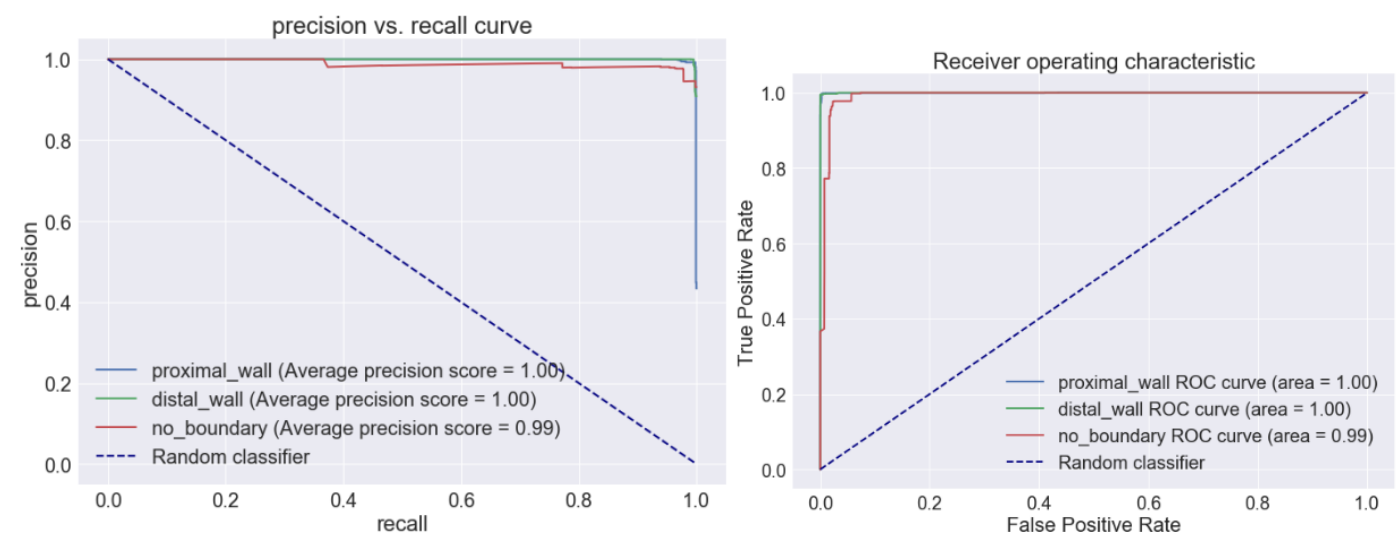

\section{CNN}
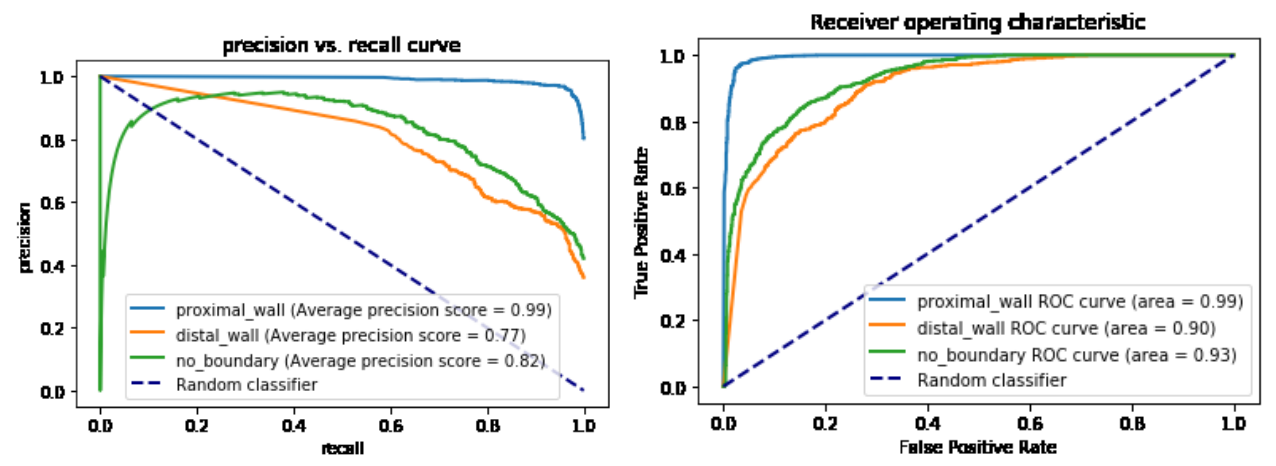

\section{Performance of machine learning algorithm in Phantom A}

\section{LDA}



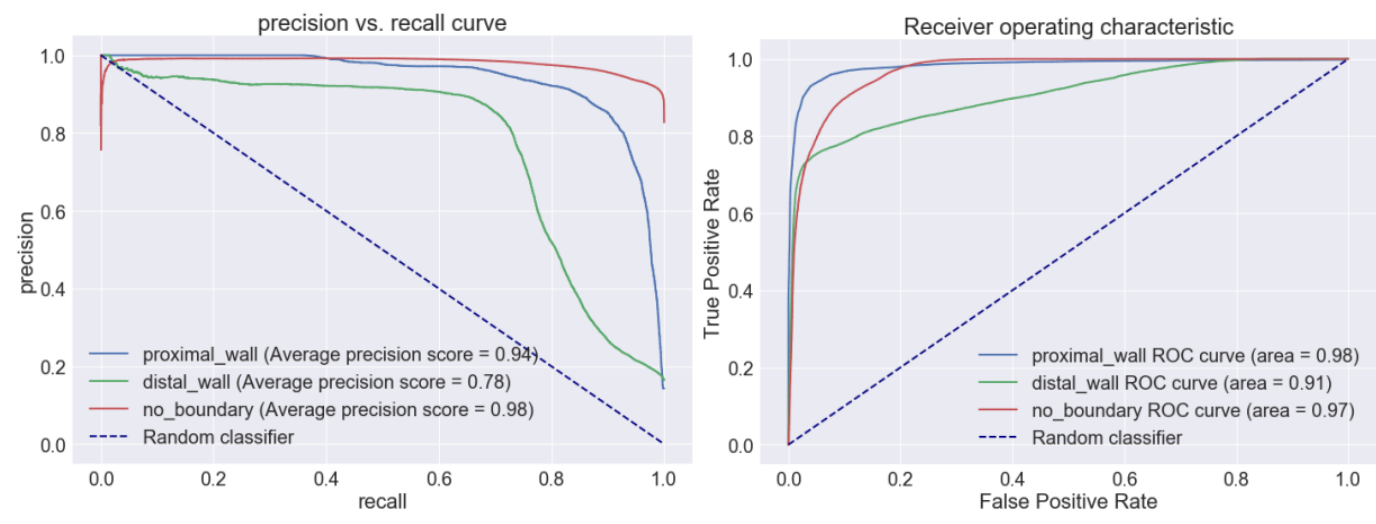

\section{KNN}
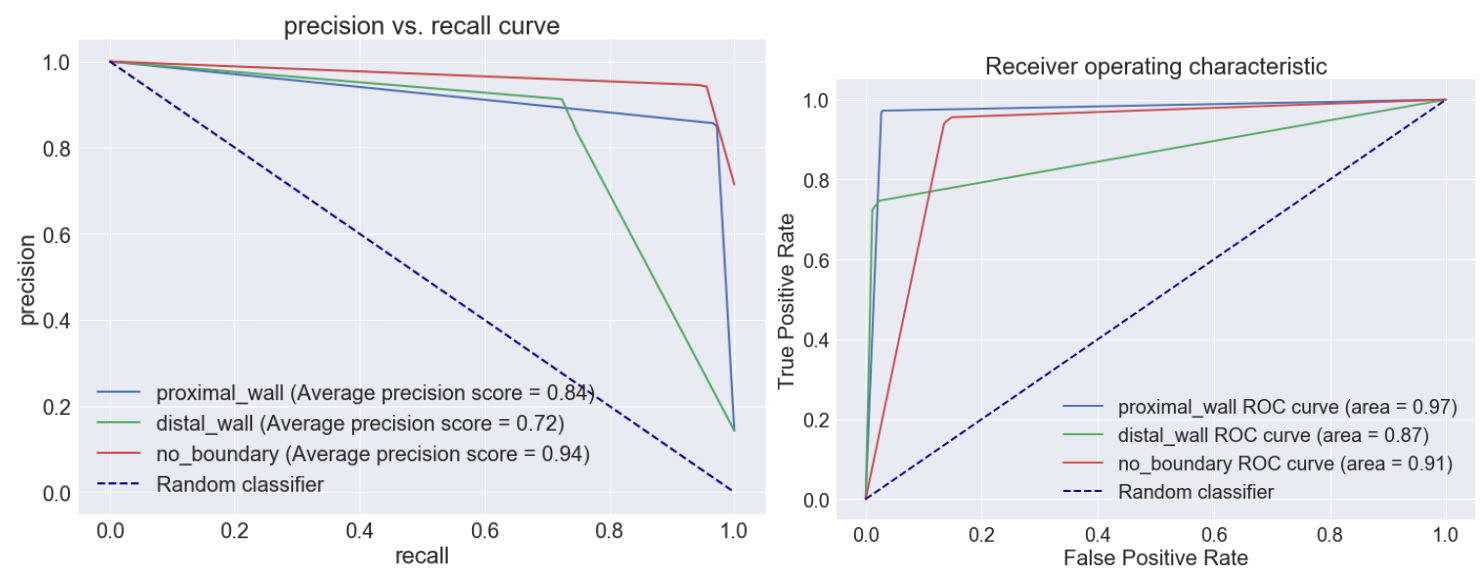

RF 

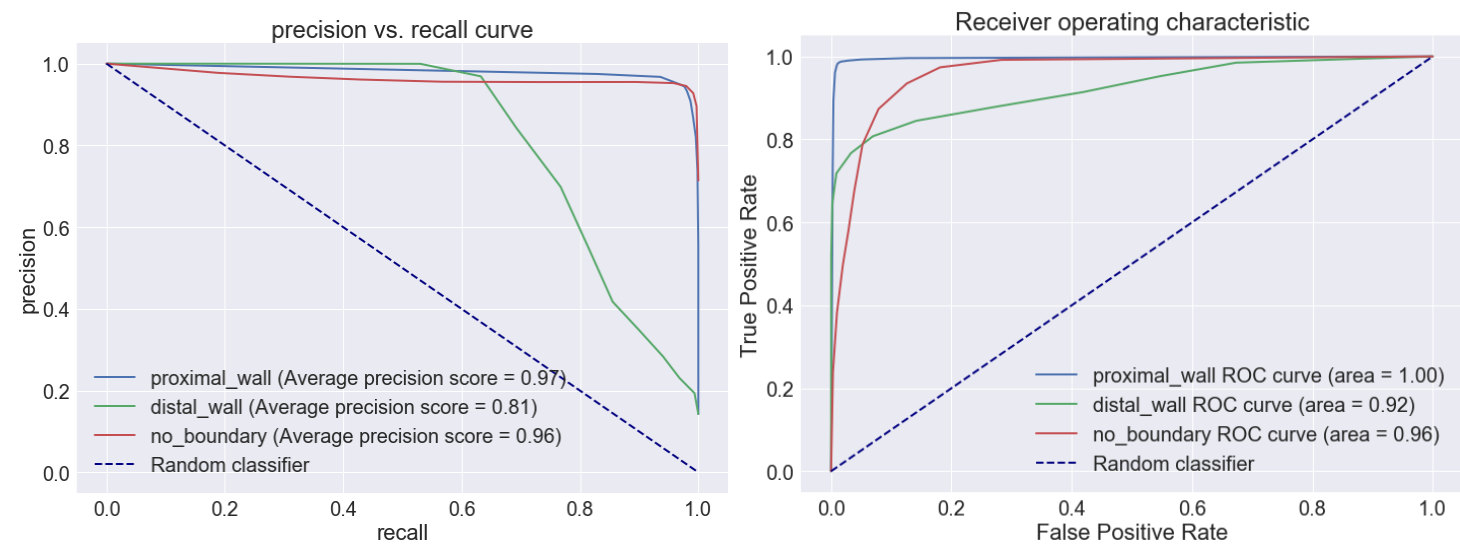

\section{XGB}
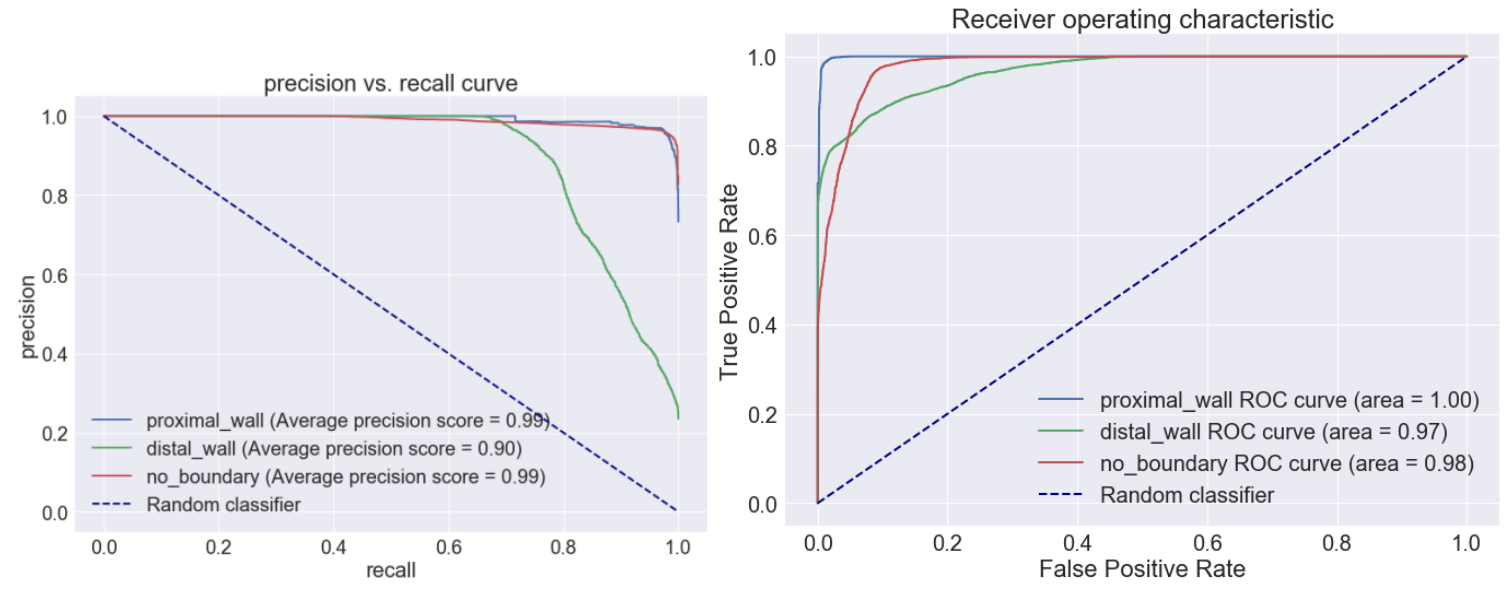

\section{CNN}



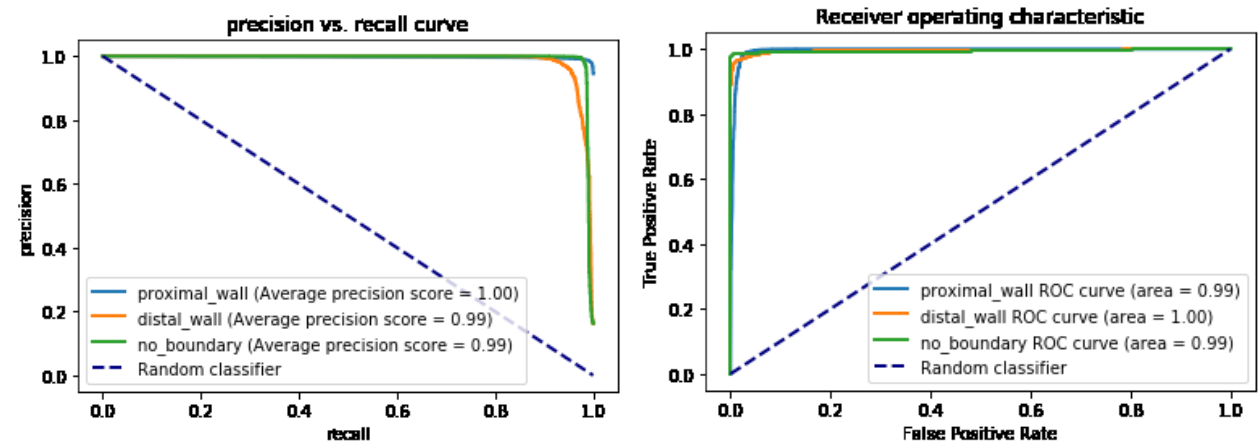

\section{Performance of machine learning algorithm in Phantom A}

\section{LDA}
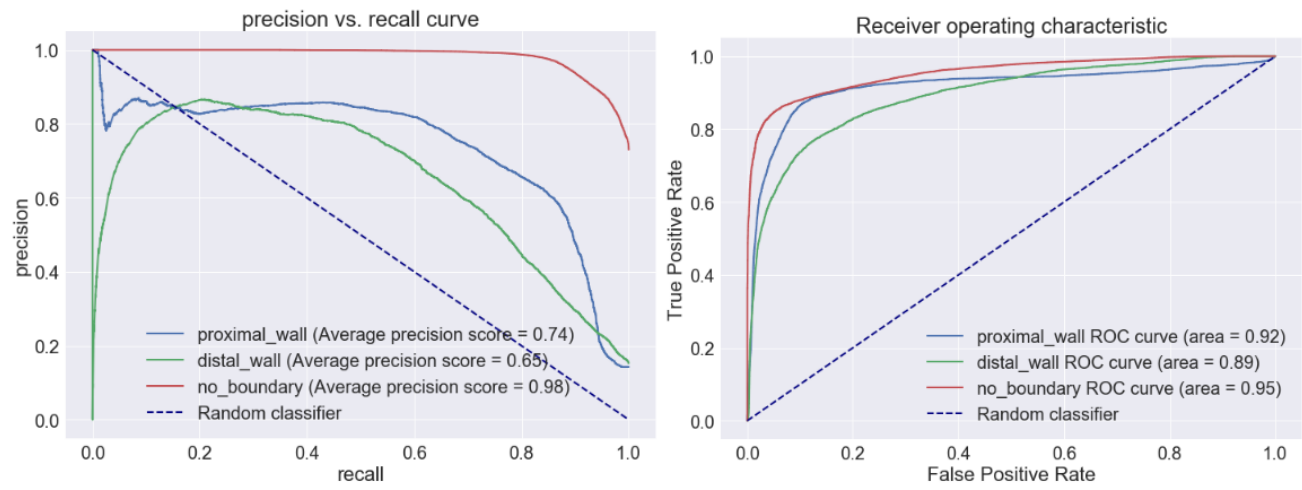

KNN 

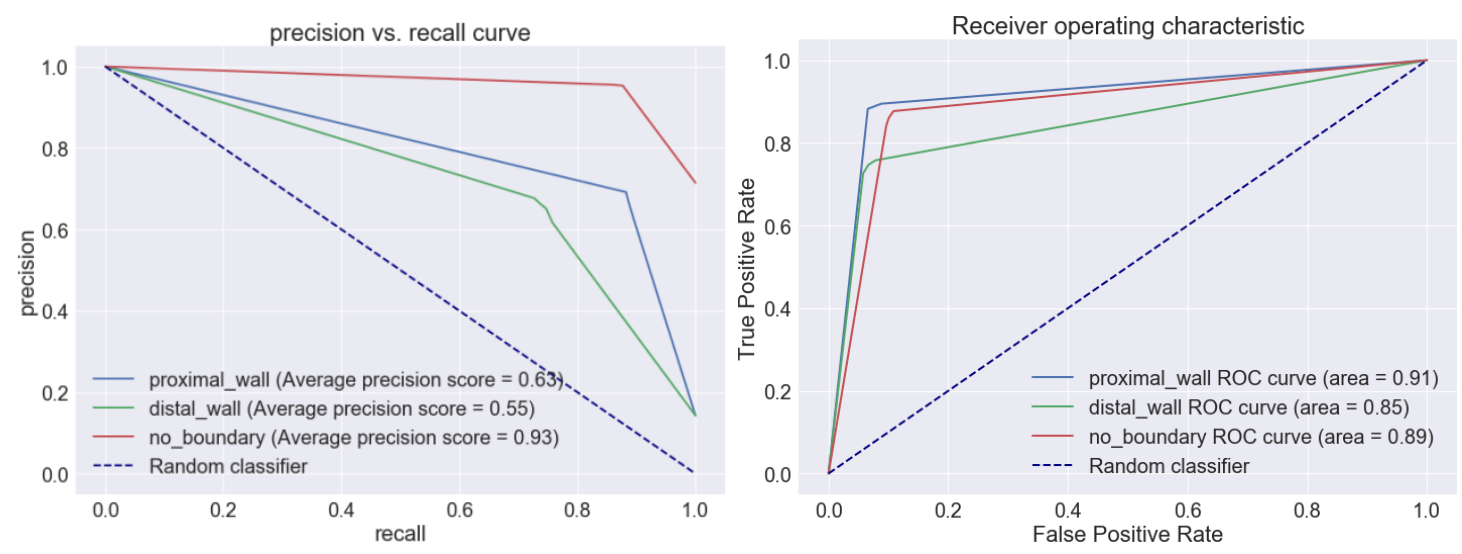

RF
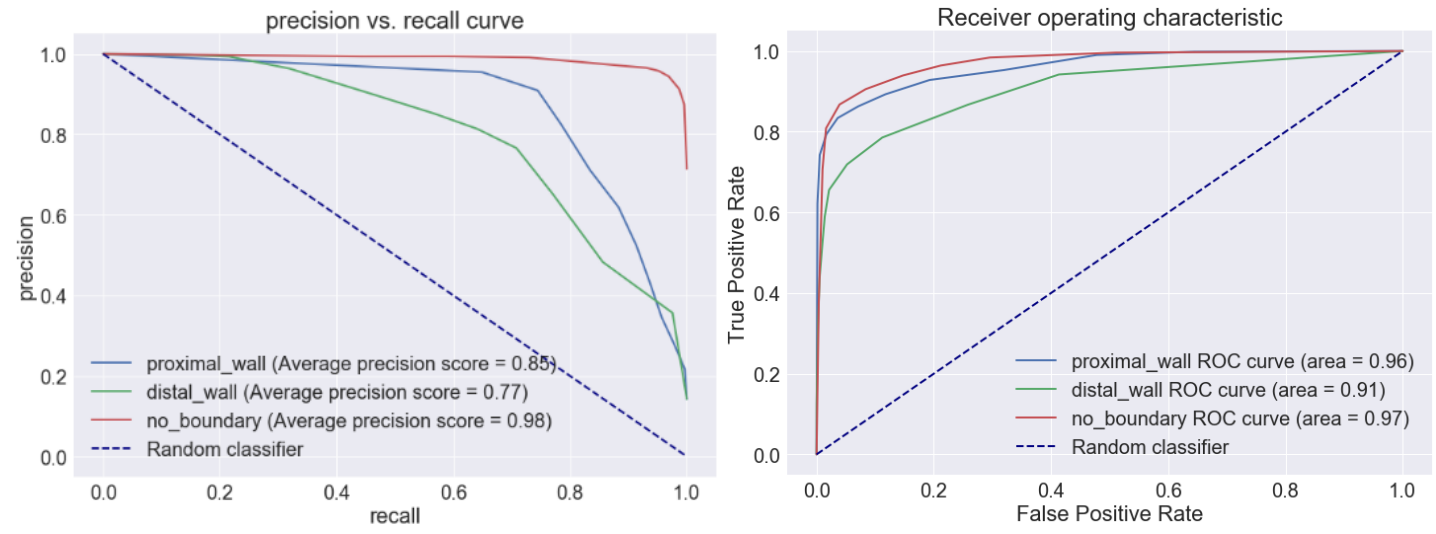

XGB 

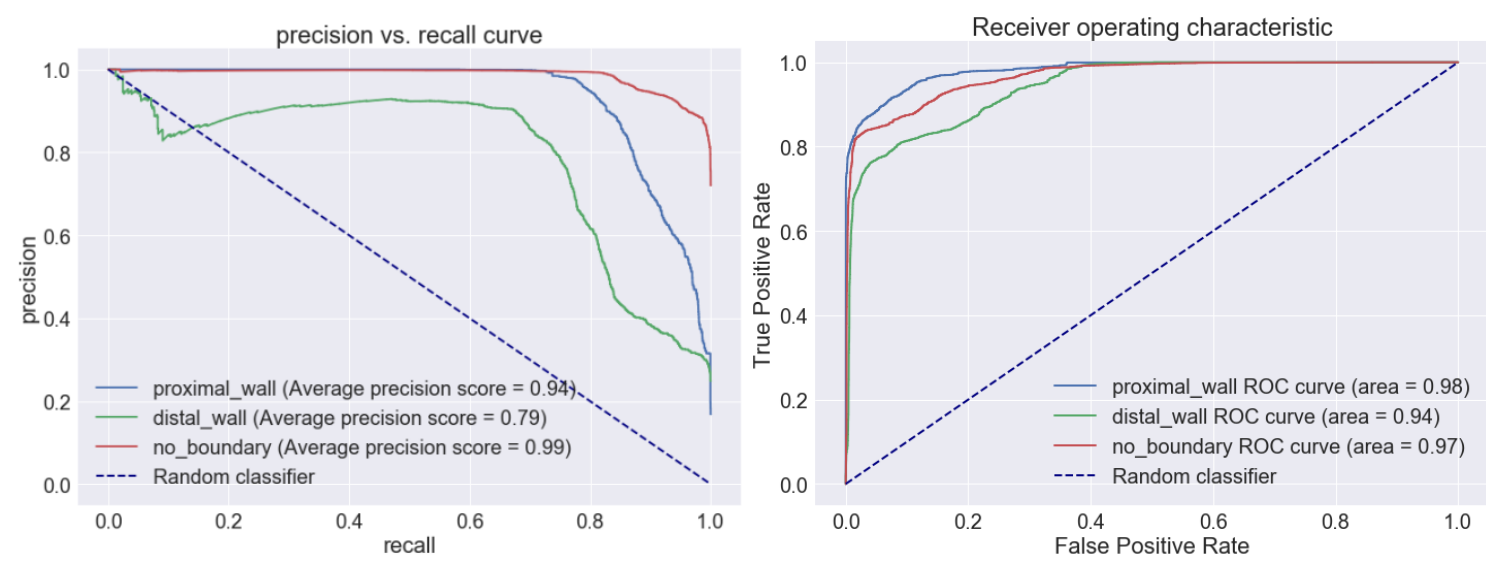

\section{CNN}
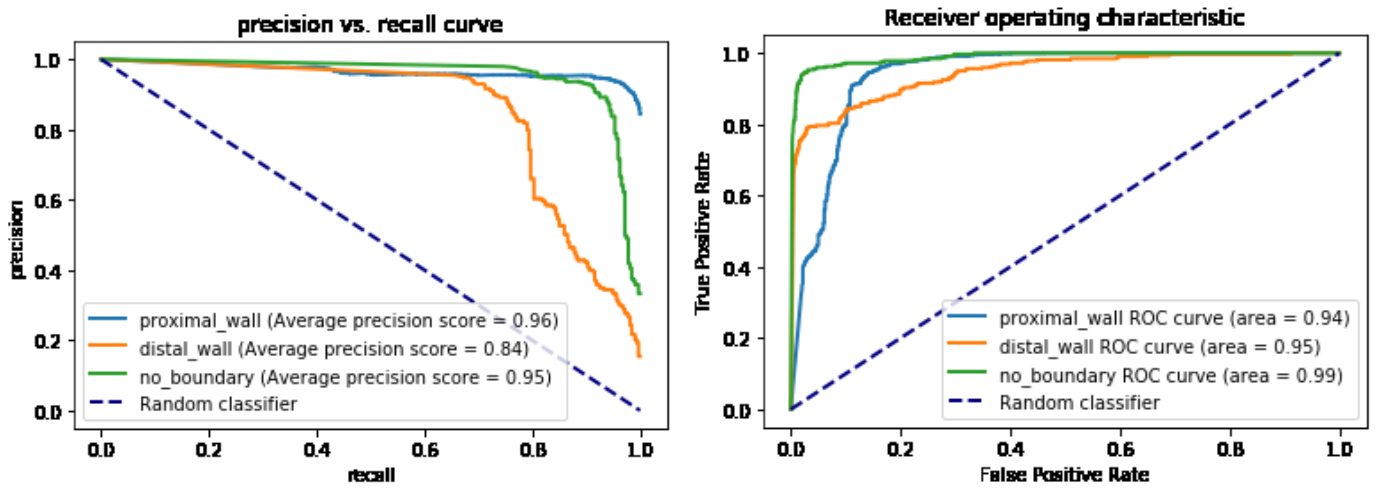

\section{Performance of machine learning algorithm in Human in-vivo from flexible}

\section{CNN}



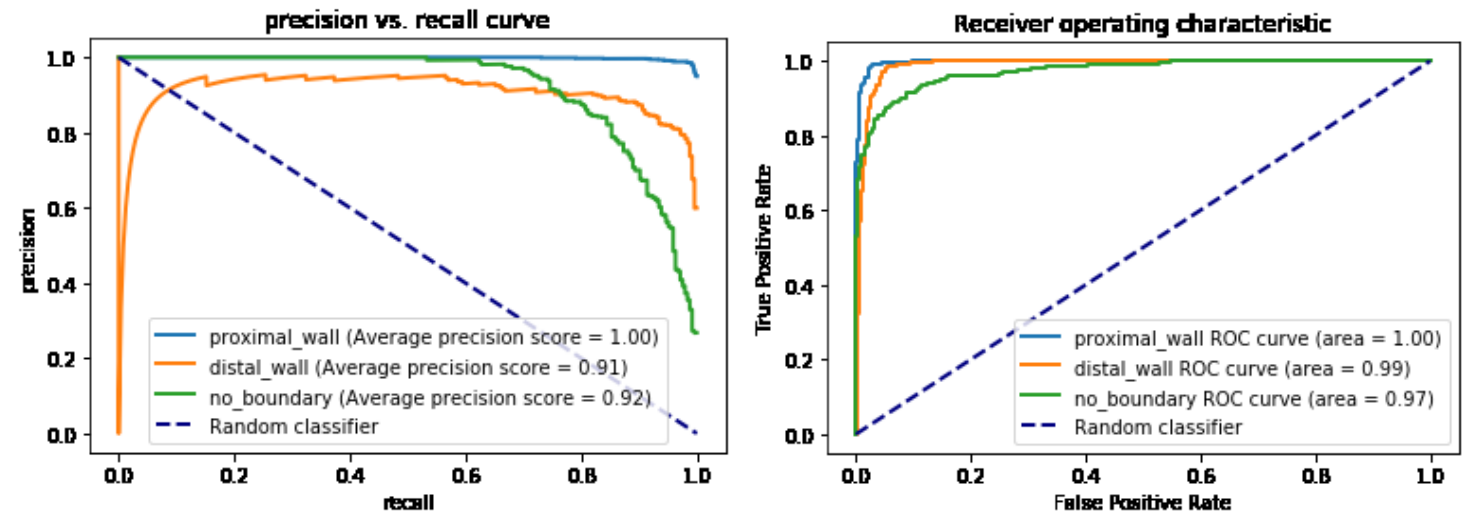

Measurement of the diameter using PICUS clinical ultrasound system

Human test subject 2
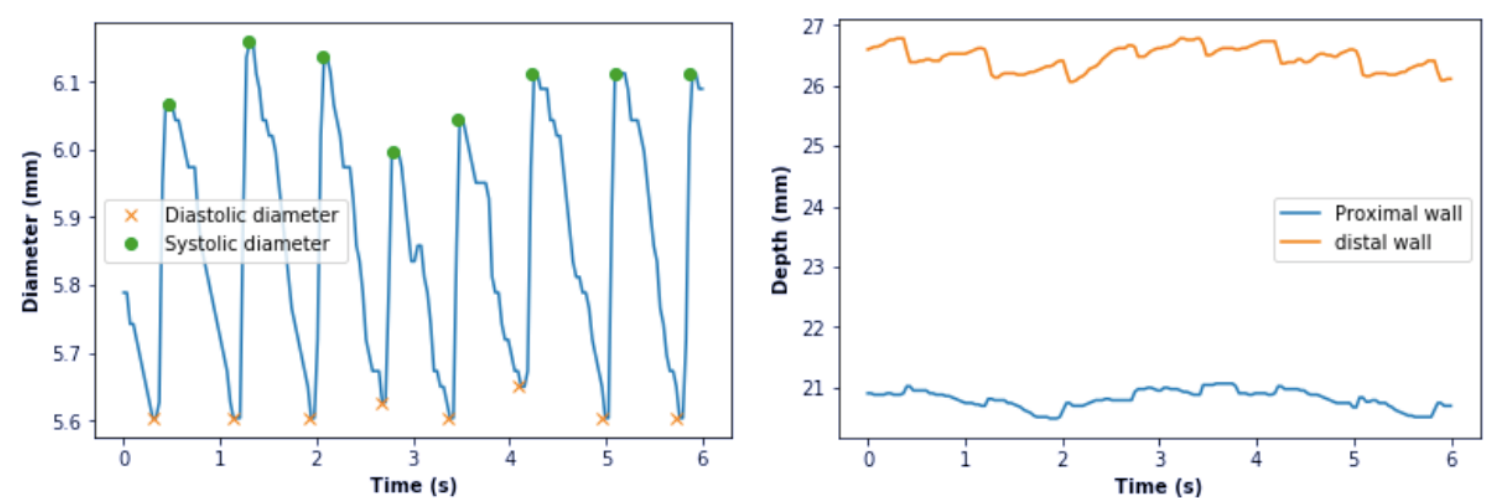


\begin{tabular}{|c|c|c|}
\hline Cardiac cycle & Diastolic diameter in mm & Systolic diameter in mm \\
\hline 1 & 5.604 & 6.066 \\
\hline 2 & 5.604 & 6.158 \\
\hline 3 & 5.604 & 6.136 \\
\hline 4 & 5.627 & 5.997 \\
\hline 5 & 5.604 & 6.043 \\
\hline 6 & 5.650 & 6.112 \\
\hline 7 & 5.604 & 6.112 \\
\hline 8 & 5.604 & 6.112 \\
\hline Mean \pm Std & $5.613 \pm 0.016$ & $6.092 \pm 0.050$ \\
\hline
\end{tabular}


Measurement of the diameter using WUS system
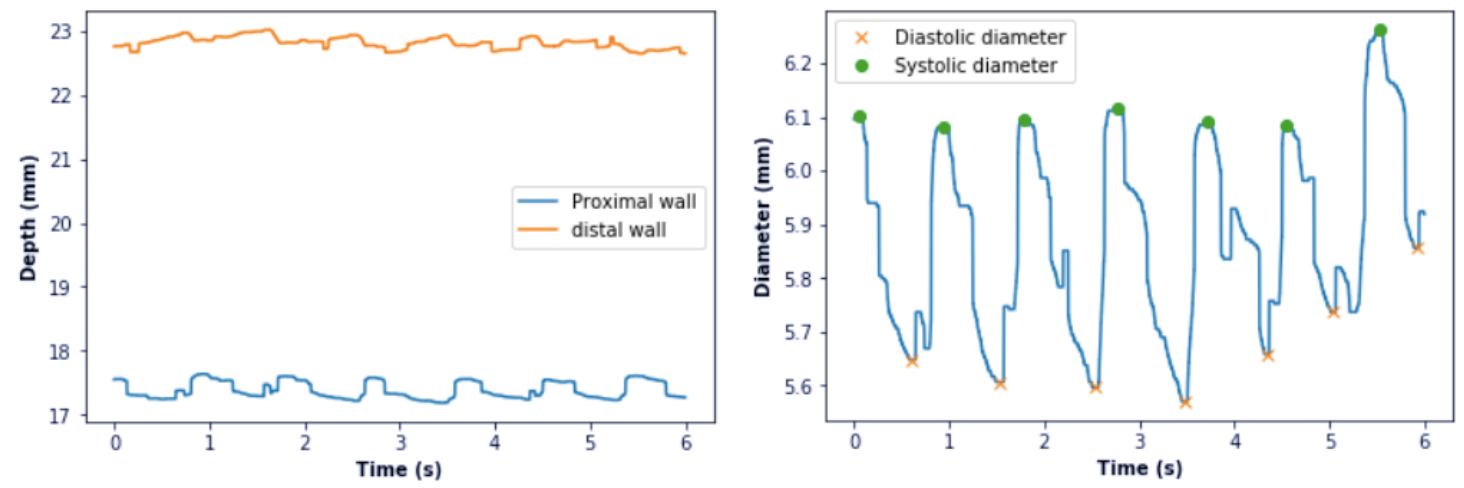

\begin{tabular}{|c|c|c|}
\hline Cardiac cycle & Diastolic diameter in $\mathbf{m m}$ & Systolic diameter in mm \\
\hline 1 & 5.648 & 6.100 \\
\hline 2 & 5.606 & 6.080 \\
\hline 3 & 5.596 & 6.095 \\
\hline 4 & 5.570 & 6.116 \\
\hline 5 & 5.659 & 6.090 \\
\hline 6 & 5.737 & 6.085 \\
\hline 7 & 5.856 & 6.262 \\
\hline Mean \pm Std & $5.668 \pm 0.092$ & $6.119 \pm 0.060$ \\
\hline
\end{tabular}

Difference between WUS and PICUS system 


\begin{tabular}{|c|c|c|}
\hline & $\begin{array}{c}\text { Mean diastolic diameter } \\
(\mathbf{m m})\end{array}$ & $\begin{array}{c}\text { Mean systolic diameter } \\
(\mathbf{m m})\end{array}$ \\
\hline PICUS & 5.613 & 6.092 \\
\hline WUS & 5.668 & 6.119 \\
\hline Difference & 0.055 & 0.027 \\
\hline
\end{tabular}

